\title{
Multimessenger Approaches to Exploring Dense Matter in Neutron Stars
}

\author{
Dissertation \\ zur Erlangung des Doktorgrades \\ der Naturwissenschaften \\ vorgelegt beim Fachbereich Physik \\ der Johann Wolfgang Goethe-Universität \\ in Frankfurt am Main \\ von \\ Lukas R. Weih \\ aus Seligenstadt
}

Frankfurt am Main (2021)

(D30) 
vom Fachbereich Physik der

Johann Wolfgang Goethe-Universität als Dissertation angenommen.

Dekan: Prof. Dr. Harald Appelshäuser

Gutachter: Prof. Dr. Luciano Rezzolla (Goethe-Universität, Frankfurt am Main)

Prof. Dr. Jürgen Schaffner-Bielich (Goethe-Universität, Frankfurt am Main)

Prof. Dr. Konstantinos Kokkotas (Eberhard-Karls-Universität, Tübingen)

Datum der Disputation: 20. Jul. 2021 


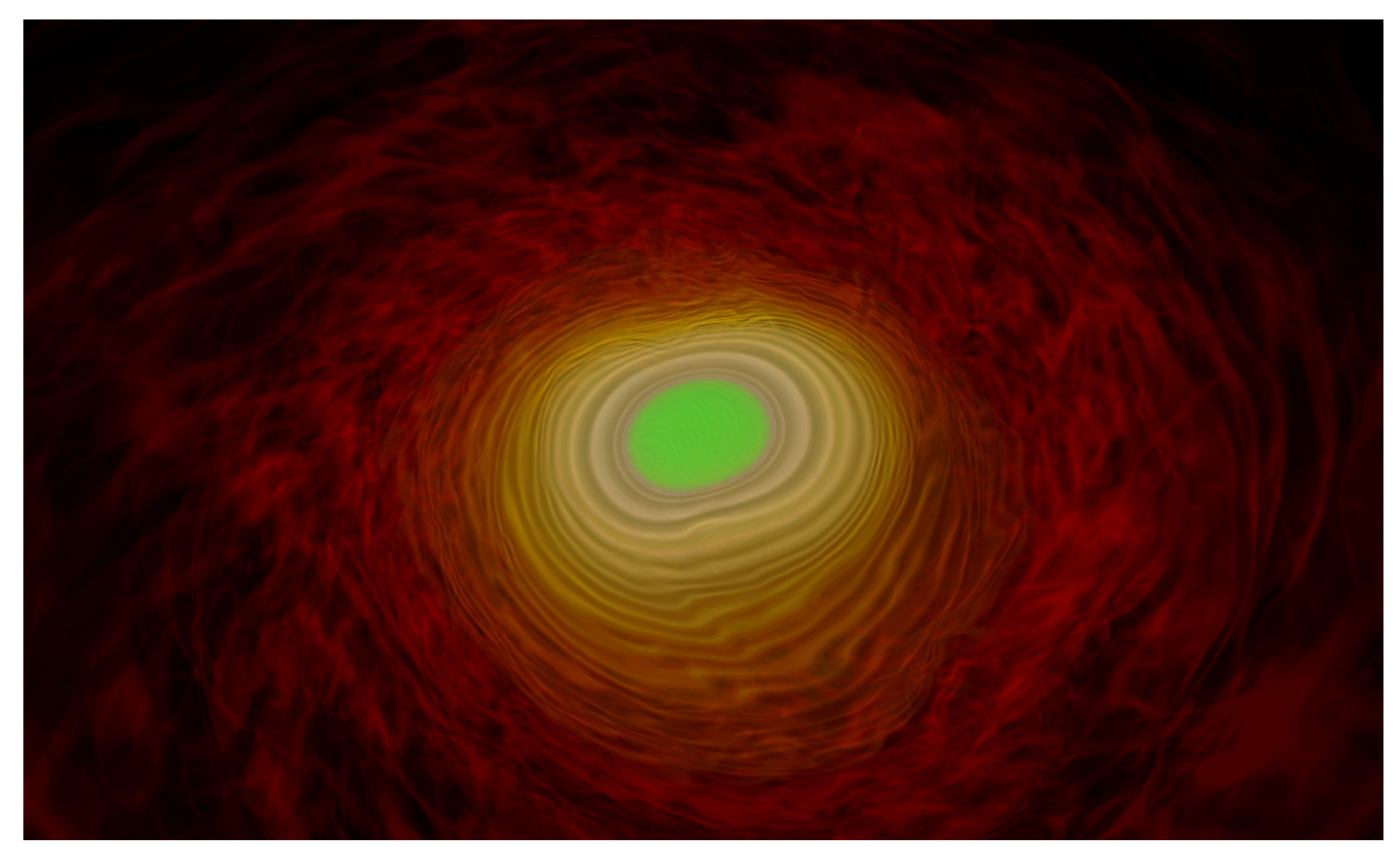

Rendering of the rest-mass density of a neutron star merger remnant, which forms a stable quark core (green). The full simulation leading to this system can be seen as flip-book visualization in the top-right corner of this Thesis. 


\section{Abstract}

High-energy astrophysics plays an increasingly important role in the understanding of our universe. On one hand, this is due to ground-breaking observations, like the gravitational-wave detections of the LIGO and Virgo network or the black-hole shadow observations of the EHT collaboration. On the other hand, the field of numerical relativity has reached a level of sophistication that allows for realistic simulations that include all four fundamental forces of nature. A prime example of how observations and theory complement each other can be seen in the studies following GW170817, the first detection of gravitational waves from a binary neutron-star merger. The same detection is also the chronological starting point of this Thesis. The plethora of information and constraints on nuclear physics derived from GW170817 in conjunction with theoretical computations will be presented in the first part of this Thesis. The second part goes beyond this detection and prepares for future observations when also the high-frequency postmerger signal will become detectable. Specifically, signatures of a quark-hadron phase transition are discussed and the specific case of a delayed phase transition is analyzed in detail. Finally, the third part of this Thesis focuses on the inclusion of radiative transport in numerical astrophysics. In the context of binary neutron-star mergers, radiation in the form of neutrinos is crucial for realistic long-term simulations. Two methods are introduced for treating radiation: the approximate state-of-the-art two-moment method (M1) and the recently developed radiative Lattice-Boltzmann method. The latter promises to be more accurate than M1 at a comparable computational cost. Given that most methods for radiative transport or either inaccurate or unfeasible, the derivation of this new method represents a novel and possibly paradigm-changing contribution to an accurate inclusion of radiation in numerical astrophysics. 


\section{Acknowledgements}

I would like to express my sincere gratitude to all the people without which the completion of this Thesis would not have been possible.

First of all, I thank Luciano Rezzolla who kindly accepted me into his group. I thank him for his guidance, insightful discussions, and invaluable advice. Over the years he kept challenging me and pushed me beyond my own boundaries. Although at times I was reluctant to carry out the many projects he suggested, in the end it always turned out that I greatly benefited from his insistence and that this was exactly the kind of challenge I was looking for when deciding to join his RelAstro group. Thank you for the many challenges and for making me a scientist. Keep insisting!

I am also grateful to Jürgen Schaffner-Bielich for agreeing not only to referee this Thesis, but to also be part of my PhD committee. Starting my Bachelor Thesis with him gave me the taste for more astrophysics.

During my four years in the RelAstro group I had the pleasure to meet a number of great people, in fact, some of the brightest minds I have come to know. A special mention deserves Elias Most, who from the very beginning guided me and acted as a mentor and friend. It is not a coincidence that he appears on almost all my publications and I feel honored to have published scientific results with someone who will become without doubt one of the greatest scientists in the future. I am also thankful to Alessandro Gabbana, who introduced me to the Lattice Boltzmann method. It was a pleasure to work with him side by side and to together explore pathways that no one explored before. A special thanks also goes to Hector Olivares. Without his expertise in BHAC I would still be trying to find the right place for implementing my routines.

I also thank all other present and past members and guests of the group who maybe without even knowing at some point contributed to this Thesis by the many discussions we had over lunch or otherwise. In no particular order I thank Matthias Hanauske, Jens Papenfort, Samuel Tootle, Zhenyu Zhu, Frederike Kubandt, Alejandro Cruz-Osorio, Michail Chabanov, Christian Ecker, Luke Bovard, Jonas Köhler, Cosima Breu, Federico Guercilena, Oliver Porth, Jan Röder, Sercan Cikintoglu, Antonio Figura, Sven Köppel, Yosuke Mizuno, Laura Tolos, Christian Fromm, Roman Gold, Antonios Nathanail, Fabio Bacchini, Enping Zhou, Ziri Younsi, Bart Ripperda, Markus Mayer, Sabine Krasilovskaja, John Whelan, and Prashant Kocherlakota.

A special thanks goes to my collaborators from Italy, the experts on Lattice Boltzmann. Namely, I would like to thank Daniele Simeoni for his help in developing our new LB method, Lele Tripiccione for his continued advice and Sauro Succi for his infectious passion for conquering new frontiers.

While barely possible, I also maintained a private live during my $\mathrm{PhD}$ studies, where the right people helped me avoiding getting crushed like the matter inside neutron stars. A huge thanks goes to Markus Schlott, who not only made me drink coffee, but the numerous discussions with him also caused a variable speed of work on this Thesis.

Of course all this would not have been possible without my family and especially my parents. Their unconditional support - be that monetary or emotional - can always be counted on and even when divided by three seems to be inexhaustible. Gracias también a mi familia mexicana. Su hogar se convirtió en mi hogar también y fue el único lugar en el que estaba lo suficientemente lejos como para tomarme un descanso de todas las estrellas de neutrones. 
Finally, I am infinitely grateful to my beautiful wife. For always being there for me. For always caring about my well-being. For providing me with a sheer endless amount of snacks. For her unconditional love and for more than words could say. 


\section{Contents}

Abstract $\quad$ iv

Acknowledgements $\quad$ v

1 Introduction $\quad 1$

1.1 Neutron Stars ......................... 1

1.2 Binary Neutron Star Mergers . . . . . . . . . . . . . . . 3

1.3 Overview of the Thesis ................... 6

2 Basic Equations $\quad 10$

2.1 General Relativity ................... . 10

2.1.1 The Einstein Field Equations . . . . . . . . . . . . . . . 10

2.1.2 Numerical Relativity . . . . . . . . . . . . . . . . 12

2.1.3 Gravitational Waves .................... 19

2.2 General Relativistic Hydrodynamics . . . . . . . . . . . . . . . . 21

2.3 Radiative Transport . . . . . . . . . . . . . . . . . . 26

2.3.1 The Radiative Transfer Equation . . . . . . . . . . . . . . . . 26

2.3.2 Solving the Radiative Transfer Equation . . . . . . . . . . . 28

2.3.3 Coupling between Radiation and Fluid . . . . . . . . . . . 32

3 Constraining the Equation of State of Nuclear Matter 35

3.1 Theoretical Neutron Star Models . . . . . . . . . . . . . . . 35

3.1.1 The Tolman-Oppenheimer-Volkoff equation . . . . . . . . . . 36

3.1.2 Soft and Stiff Neutron Stars: the Equation of State and Tidal

Deformability ................. 37

3.1.3 Rotation and Stability . . . . . . . . . . . . . . . 42

3.2 Constraints from the Gravitational Wave

Event GW170817 . . . . . . . . . . . . . . . . . . . . 44

3.2.1 An Upper Limit on the Maximum Mass of Neutron Stars . . . 45

3.2.2 From the Tidal Deformability to the Neutron Star Radius . . . 48

3.3 Optimal Mass Ranges for Future Constraints . . . . . . . . . . . . . . 51

3.3.1 Comparison to Results from the NICER Mission . . . . . . 57

3.4 Summary and Outlook .................... . . 58

4 Quark-Hadron Phase Transitions in Binary Mergers 60

4.1 The Postmerger Gravitational-Wave Signal . . . . . . . . . . . . . . . 60

4.2 Simulation Setup . . . . . . . . . . . . . . . . . 62

4.3 Overall Simulation Results. . . . . . . . . . . . . . . . 65

4.4 The Delayed-Phase-Transition Scenario . . . . . . . . . . . 67

4.4.1 Thermodynamic Properties of a Hypermassive Hybrid Star . . 67

4.4.2 Gravitational-Wave Signature of a Stable Hypermassive Hybrid Star ..................... . . 70

4.5 Summary and Outlook ...................... 72

5 Radiative Transport in Numerical Astrophysics $\quad 74$

5.1 The Two-Moment Scheme for General-Relativistic Radiation Hydrodynamics ........................ . . 75

5.1.1 Implementation of the M1 Scheme . . . . . . . . . . 76 
5.1 .2 Code Tests . . . . . . . . . . . . . . . . 87

5.1 .3 The Radiative Michel Solution . . . . . . . . . . . . . . . . 94

5.2 Beyond Moments: the Lattice Boltzmann Method . . . . . . . . . . . 102

5.2.1 Classical Lattice Boltzmann in a Nutshell . . . . . . . . . . . 102

5.2.2 Derivation of the Lattice Boltzmann Method for Radiative Trans-

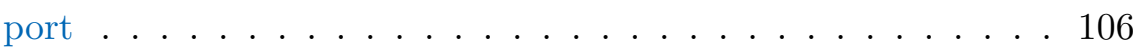

5.2 .3 Code Tests and Comparison to M1 . . . . . . . . . . . 113

5.2 .4 Relativistic Jets . . . . . . . . . . . . . . . . . . 121

5.3 Summary and Outlook . . . . . . . . . . . . . . . 125

$\begin{array}{llr}6 & \text { Conclusions } & 127\end{array}$

$\begin{array}{ll}\text { A Units and Unit Conversions } & 130\end{array}$

B Emissivity and Opacities in the Laboratory Frame 132

C Link Between Microscopic and Macroscopic Parameters $\quad 134$

C.1 Chapman-Enskog Analysis . . . . . . . . . . . . . . . . . . . 134

C.2 Numerical Fit for Scattering Opacities . . . . . . . . . . . . 136

$\begin{array}{lr}\text { Bibliography } & 138\end{array}$

German Summary (Zusammenfassung) 172

$\begin{array}{lr}\text { Curriculum Vitae } & 178\end{array}$

$\begin{array}{lr}\text { Selbstständigkeitserklärung } & 182\end{array}$ 


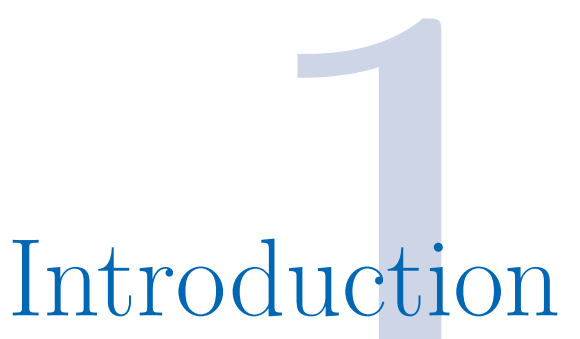

High-energy astrophysics plays an increasingly important role in the understanding of the fundamental laws of our universe. This is due to worldwide collaborations that push the frontiers of astrophysics. One of these collaborations is the LIGO and Virgo network of gravitational-wave detectors (LVC) that for the first time recorded the gravitational-wave signal of a coalescing pair of black holes in 2015 (Abbott et al., 2016). This event with the name GW150914 marks a milestone because it not only confirms Einstein's hundred year old hypothesis of gravitational waves, but also because for the first time our universe has been observed through the fundamental force of gravity.

The second milestone was the millimeter-wavelength observation of the Event Horizon Telescope Collaboration (EHT) resulting in the first spatially resolved image of the shadow of a black hole in the center of the galaxy M87 (Event Horizon Telescope Collaboration et al., 2019a,b,c,d,e,f).

Both of these events have in common that their understanding and interpretation was assisted by theoretical computations. More precisely, numerical high-energy astrophysics, i.e., the simulation of neutron star or black-hole systems, provided templates for gravitational-wave patterns, synthetic images of a black-hole shadow, and the general knowledge of how these systems behave. This Thesis is a contribution to this theoretical understanding. On one hand, theoretical models are used to interpret some of these landmark detections. On the other hand, new models, simulations, and methods are presented with the aim to prepare for future detections in the same way as the field of numerical relativity has been preparing over the last 20 years for the revolutionary detections of the last decade.

The astrophysical background of this Thesis is briefly introduced in the following two sections. Furthermore, the phenomenology of binary neutron star mergers is presented both from an observational and theoretical point of view. This includes an overview of the current state of research in the fields of numerical relativity, neutron stars, and the broader context of high-energy astrophysics. It will become clear how this Thesis aligns itself within this context. Finally, Sec. 1.3 presents the outline of this Thesis.

\subsection{Neutron Stars}

The most prominent objects in this Thesis are by far neutron stars. Neutron stars are classified as compact objects and an extensive overview of these stars can be found in Glendenning (2000); Rezzolla et al. (2018b); Schaffner-Bielich (2020).

Neutron stars represent the final evolution stage of massive stars. When the degenerate core of a star with a mass larger than $\sim 8 M_{\odot}$ exceeds the Chandrasekhar limit, a type II supernova is triggered (Carroll \& Ostlie, 2007). This is because the 
core's electron degeneracy pressure can no longer balance the immense gravitational pull. Due to the collapse the core's density further increases causing its protons to turn into neutrons via electron capture. These neutrons will eventually become degenerate and cause the core to stiffen. The infalling material is then reflected by the stiff core and thus an outwards propagating shock-wave is created. This shockwave stalls when it meets the infalling outer core. It has long been unknown how to further power this shock until neutrinos have been proposed for a possible explosion mechanism (Colgate \& White, 1966; Bethe \& Wilson, 1985). These neutrinos are produced in the hot and dense core and their energy drives the shock outwards. When the shock meets the stellar surface, the star's outer layers are expelled leaving behind the core as proto-neutron star (see Janka et al., 2007, for more details). Another possibility for producing a neutron star is via an accreting white dwarf resulting in a type Ia supernova. Main-sequence stars with a mass higher than $\sim 25 M_{\odot}$ will not result in a neutron star and instead directly collapse to a black hole.

The newly born neutron star has a typical mass of $\sim 1.4 M_{\odot}$ with an upper limit that is still discussed (see Chap. 3), but can safely be assumed to be less than $3 M_{\odot}$ and more than $1.97 M_{\odot}$ (Demorest et al., 2010; Antoniadis et al., 2013). Recently, this latter value could be tightened to $2.14 M_{\odot}$ through the observation of PSR J0740+6620 (Cromartie et al., 2020), however, only within a large uncertainty interval. At the same time, neutron stars have radii around $11-14 \mathrm{~km}$, making them the densest objects in our universe with densities up to $\sim 6.5$ times nuclear saturation density (Weih et al., 2019). Furthermore, they exhibit magnetic fields with an amplitude $\gtrsim 10^{13} \mathrm{G}$ and have rotation periods of milliseconds (Hessels et al., 2006). The combination of these magnetic fields and their rapid rotation provides a clear observational signature through which a neutron star can be detected; the misalignment of the magnetic field and the rotation axis causes a lighthouse effect, i.e., a pulse of radiation that can be detected at every revolution at the moment when the magnetic field points towards our detectors (Lorimer \& Kramer, 2012). These rapid pulses are eponymous for the name pulsar.

These extreme conditions make neutron stars the ideal laboratory for not only gravity, but also microphysics (see Baiotti \& Rezzolla, 2017, for a review). The outer layers, specifically the crust, where matter reaches densities up to $10^{14} \mathrm{~kg} \mathrm{~m}^{-3}$, is relatively well understood (Lattimer \& Prakash, 2001) and consists of neutron-rich nuclei. At higher densities, i.e., in the inner crust, the neutron drip begins and free neutrons start to appear. At the bottom of this inner crust, i.e., at densities around nuclear saturation density, no more nuclei can be found. Below this region is the neutron-star core, which is divided into an outer and inner core. The outer core consists of mostly free neutrons and a few free protons and relativistic electrons. The inner core, on the other hand, is only poorly understood and many models exist attempting to describe this region with densities up to $\sim 6.5$ times nuclear saturation density (see Oertel et al., 2017, for a review).

This ignorance gives rise to an entire zoology of exotic objects that are all described in detail in Schaffner-Bielich (2020). A common assumption is that hyperons are present in the neutron-star core (Kiuchi et al., 2012; Tolos et al., 2017a; Fortin et al., 2018). More extreme, but still one of the most common and plausible ideas is that of hybrid stars (see for example Most et al., 2018; De et al., 2018; Montaña et al., 2019; Alvarez-Castillo et al., 2019; Christian et al., 2018; Annala et al., 2020). Assuming that the matter undergoes a phase transition in the dense inner core, a hybrid star is a neutron star whose center consists of deconfined quarks (Collins \& Perry, 1975). This type of star will also be discussed in Chap. 4 of this Thesis. Going a step further, some studies even model quark stars (Drago et al., 2007), i.e., compact stars that 
completely consist of free quarks, or stars with a color superconducting core (Alford \& Sedrakian, 2017). Holographic models are also being used for describing neutron star matter (Annala et al., 2018a; Ecker et al., 2020).

All these models require the comparison of theoretical calculations with observations. Accreting neutron stars can be observed via thermonuclear bursts in the X-ray spectrum (Lewin et al., 1993). Unfortunately, these observations are model dependent and lack in accuracy. The resulting microphysical information that is extracted from these observations is thus equally inaccurate (Özel et al., 2016; Guillot et al., 2013; Lattimer \& Steiner, 2014; Nättilä et al., 2017). Recently, the Neutron Star Interior Explorer (NICER) yielded the most accurate direct neutron star measurements to date (Bogdanov et al., 2019a,b). Future missions of this type promise accurate measurements of neutron-star masses and radii. Indirect observations via Shapiro delay (Shapiro, 1964) are also routinely used for measuring neutron-star masses with great precision.

Another and possibly the most promising avenue, however, is not based on any electromagnetic signal and instead relies on a different force: gravity. Over the past view years gravitational-wave measurements have become a new viable option for observing neutron stars as will be discussed in the following section.

\subsection{Binary Neutron Star Mergers}

Neutron stars are not only found isolated, but also in binary systems. In such a binary, the two companions orbit each other and curve spacetime in such a way that gravitational waves are emitted. This emission and the corresponding energy and angular momentum loss of the system causes both stars to get closer (Peters, 1964) until they eventually merge. Such binary neutron star mergers have long been studied via simulations with more and more sophisticated numerical tools (see Baiotti \& Rezzolla, 2017; Dietrich et al., 2020b, for reviews). These simulations show that in general three distinct scenarios have to be distinguished. These scenarios are characterized by the outcome of the merger, which depends on the underlying equation of state (EOS) and the total binary mass. They are summarized schematically in Fig. $1.1^{1}$.

The top panels show the case of a prompt collapse to a black hole. For high masses and/or a soft EOS (see Chap. 3 for details on softness and stiffness) the two neutron stars will merge and immediately (within the free-fall time) collapse to a black hole. The outcome will than be a black hole with an optically thick torus around it. Interestingly, the threshold mass to prompt collapse can be related to unique properties of the EOS in a quasi-universal manner (Bauswein et al., 2013; Koeppel et al., 2019). The corresponding gravitational-wave signal in Fig. 1.1 consists of a long inspiral signal (green shaded) and a short merger signal (yellow shaded) that is directly followed by the ring-down signal (red) corresponding to the collapse.

Next, there is the delayed-collapse scenario shown in the middle panels of Fig. 1.1. Here, the total binary mass is smaller so that the merger leads to a meta-stable object called hypermassive neutron star (HMNS). Inside this HMNS, the density increases beyond those of the original two neutron stars. During the merger, shock-waves also heat up the matter reaching temperatures up to $\sim 100 \mathrm{MeV}$ in a ring-like structure

\footnotetext{
${ }^{1}$ Despite the general cases depicted in Fig. 1.1 an entire new set of possibilities arises when also including the possibility of a phase transition during the merger. This will be discussed extensively in Chap. 4 and we here focus only on the "classic" cases of purely hadronic mergers. Furthermore, the description is true for equal-mass mergers and might differ for high mass ratios.
} 


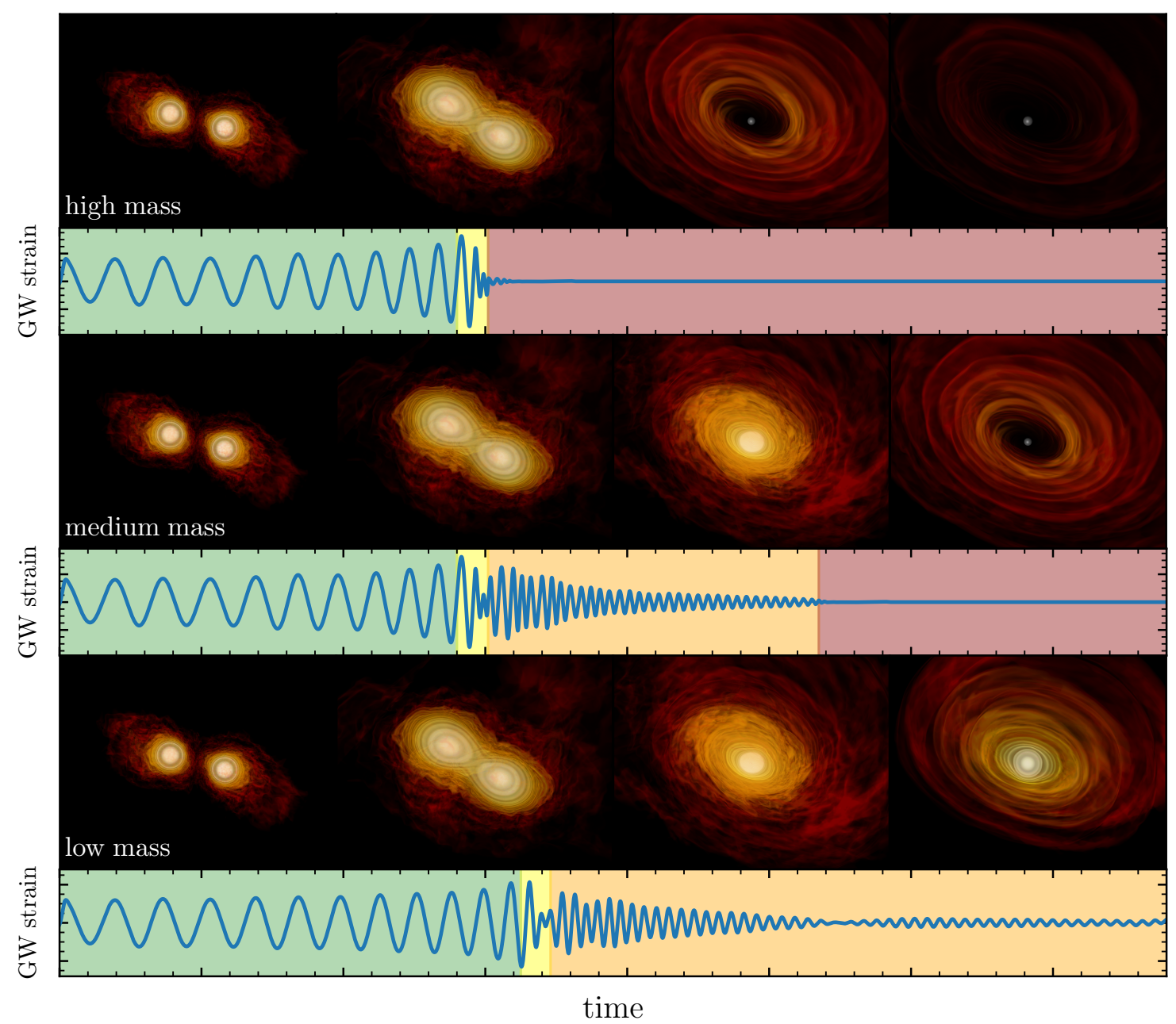

FIGURE 1.1: Schematic overview of the possible outcomes of a binary neutron star merger. The three panels show top to bottom the prompt collapse, delayed collapse, and stable HMNS scenario, which depend on the total binary mass. For each scenario four renderings are shown denoting the inspiral, merger, postmerger and long-term phase. In the corresponding gravitational-wave (GW) signal - shown below each scenario - the inspiral signal is marked green, the merger yellow, the postmerger orange and the ringdown signal following the collapse to a black hole is marked red.

around the HMNS core (see Hanauske et al., 2017a; Perego et al., 2019, for details on the thermodynamic properties of these remnants). During the following violent postmerger phase, the frequency of the gravitational waves is increased $(\gtrsim 1 \mathrm{kHz})$ due to the increased angular velocity of the HMNS. This HMNS is differentially rotating (Kastaun \& Galeazzi, 2015; Hanauske et al., 2017a) with a slowly rotating core surrounded by a fast-rotating envelope. The outermost layers and the torus of the HMNS have a Keplerian rotation profile. This differential rotation allows the HMNS to support a large amount of mass. After redistributing its angular momentum due to viscous effects (Shibata et al., 2017) the HMNS will reach a more uniform rotation profile and eventually collapse to a black hole, again leaving behind a torus.

Finally, the bottom panels in Fig. 1.1 show a low-mass merger. In this scenario, the binary mass is low enough so that even the uniformly rotating remnant can withstand the inwards directed gravitational pull and prevent a collapse. A collapse might still happen at a later time; the rotating neutron star will spin-down through 
the continuous emission of gravitational waves. This spin-down results in a smaller centrifugal support. Crossing the stability limit (Takami et al., 2011; Weih et al., 2018) will again lead to a collapse. On the other hand, if the binary mass is low enough, even after losing all its angular momentum the neutron star might be stable. In this case, the final outcome of the merger would be a cold non-rotating isolated neutron star.

The latter two scenarios, i.e., the scenarios with an HMNS phase, both show a postmerger gravitational-wave signal (see orange shadings in Fig. 1.1). This part of the signal is characteristic for each EOS. A quasi-universal relation (Bauswein \& Janka, 2012a; Takami et al., 2014, 2015; Bernuzzi et al., 2015; Rezzolla \& Takami, 2016; Maione et al., 2017; Kawaguchi et al., 2018; Dietrich et al., 2019) connects the dominant frequency of this postmerger signal with the tidal deformability that can be measured during the inspiral (see Sec. 3.1.2 for the definition of the tidal deformability).

All this was already known long before the first ever detection of gravitational waves from a binary neutron-star merger in August 2017 (Abbott et al., 2017). This detection, dubbed GW170817, was accompanied by a short gamma-ray burst (SGRB) that was detected only $\sim 1.7$ s later (LIGO Scientific Collaboration et al., 2017). These two detections alone did not only confirm the long-standing hypothesis of neutron star mergers being connected to SGRBs (Rezzolla et al., 2011), but also heralded the beginning of the multimessenger era (Metzger, 2017b), which luckily coincided with the beginning of the work summarized in this Thesis. Furthermore, an optical glow, called kilonova $^{2}$, produced by the radioactive decay of the heavy elements synthesized in the neutron-rich merger ejecta (Just et al., 2015a) could also be observed (Drout et al., 2017; Villar et al., 2017; Cowperthwaite et al., 2017) in the UV, optical and near-infrared bands. All these different detections from this single event have led to a number of conclusions on neutron stars and dense matter, discussed in Chap. 3.

Unfortunately, LVC could only record the inspiral signal (see green shaded regions in Fig. 1.1) of GW170817. The postmerger signal could not be picked up due to the current detectors' lack of sensitivity at higher frequencies. As a consequence, the exact lifetime of the remnant is still object of discussion (Gill et al., 2019; MurguiaBerthier et al., 2020) and a clear classification as one of the three scenarios shown in Fig. 1.1 is not possible. As will be discussed in Chap. 3, however, the medium mass scenario with a meta-stable HMNS that eventually collapses, is the most likely and most agreed upon scenario.

Another factor of uncertainty concerns the long-term evolution of the merger remnant. Typical three-dimensional simulations are only able to evolve the merger product for $\sim 100 \mathrm{~ms}$ after the merger. Longer evolutions are only possible in two dimensions under the assumption of symmetry (Fujibayashi et al., 2017) or with a fixed spacetime (Fernández et al., 2019). Consequently, the long-term evolution of GW170817 is not clear and indeed the amount of inferred mass ejecta seems to differ from the predictions of numerical simulations. Typically, the ejecta is explained with two or even three components (Cowperthwaite et al., 2017; Tanvir et al., 2017; Tanaka et al., 2018). The first component is the dynamical ejecta produced during the inspiral and at merger. This ejecta is mostly restricted to the equatorial plane, is neutronrich (with an electron fraction $\lesssim 0.1$ ), and has comparatively low velocity. This will lead to the production of lanthanides, whose decay produces the red component of the kilonova (Metzger \& Fernández, 2014). This dynamical ejecta is well studied (Rosswog et al., 1999; Dietrich et al., 2017; Bovard et al., 2017; Ciolfi et al., 2017;

\footnotetext{
${ }^{2}$ A more precise name would be "macronova" (e.g., as in Rosswog et al., 2017), but "kilonova" has become the more widely used term.
} 
Most et al., 2019c) since it is produced early on during the merger. In contrast, the second component emerges from the secular ejecta, i.e., from the remnant's long-term evolution and also from the torus around the black hole when the latter is formed. This material is ejected by a magnetically or neutrino driven wind mostly along the polar directions. The neutrino radiation significantly alters the composition of the mass ejecta (Rosswog, 2005; Perego et al., 2014; Fujibayashi et al., 2017; Miller et al., 2019a) making it more proton-rich. Consequently, less lanthanides will be synthesized, so that this component has a lower opacity resulting in a blue kilonova (Metzger \& Fernández, 2014). Neutrinos might also help clearing the polar regions from baryonic matter providing a clean funnel for the SGRB to breakout (Fujibayashi et al., 2017). The study of these long-term effects requires long-term simulations on one hand and the accurate treatment of neutrinos on the other. In Chap. 5 two promising methods suitable for these kind of simulations, but also for all other types of high-energy astrophysical simulations, will be introduced.

\subsection{Overview of the Thesis}

This Thesis consists of three main parts, each broadly related to the three stages of a binary neutron-star merger: inspiral, postmerger, and long-term evolution (see Fig. 1.1). In the following we list a short overview of each chapter:

Chapter 3

This chapter focuses on constraints on the EOS of nuclear matter that could be derived from GW170817. The main ingredient for this is the detected inspiral signal. Furthermore, these gravitational-wave constraints are combined with potential constraints from direct neutron star observations.

Chapter 4

As already mentioned in the previous section, the possibility of a phase transition during the merger allows for an entire new set of possible postmerger outcomes. In Chap. 4 these possibilities are summarized and the specific case of the so-called delayed phase transition scenario is analyzed in detail.

Chapter 5

In an attempt towards accurate neutrino treatment for binary neutron -tar simulations and high-energy astrophysics in general, Chap. 5 presents two methods for radiative transport. First, the commonly used M1 method is introduced and its implementation suitable for simulations of binary neutron stars is presented. Going beyond the state-of-the-art, a new method, the radiative Lattice Boltzmann method will be introduced as a second possibility promising higher accuracy than M1 at a comparable runtime. This is shown via the study of relativistic jets as a proof-of-concept simulation.

The results of these chapters are published and peer-reviewed. Specifically, each chapter is based on the publications (a full publication list can be found in the appended $\mathrm{CV}$ ) in the following list, where (only) the author's contributions are detailed for each publication: 
Chapter 3

- Rezzolla, L., Most, E. R., \& Weih, L. R., Using Gravitational-wave Observations and Quasi-universal Relations to Constrain the Maximum Mass of Neutron Stars, Astrophys. J. Lett., 852, L25 (2018).

LRW, LR, and ERM discussed the idea and derived the estimate. LRW provided the universal relation (Fig. 2), for which he computed all neutron star models and performed the complete data analysis. LRW also produced the cartoon (Fig. 1) for explaining the method with input from LR and ERM. LRW performed the final error estimation and helped in writing of the manuscript.

- Most, E. R., Weih, L. R., Rezzolla, L., \& Schaffner-Bielich, J., New Constraints on Radii and Tidal Deformabilities of Neutron Stars from GW170817, Phys. Rev. Lett., 120, 261103 (2018).

LRW contributed to the EOS routines of the code and wrote the parallel I/O suitable for the big-data production of this study. LRW helped with the processing and analysis of the data as well as with the visual presentation of the results. LRW contributed during all stages of writing the manuscript.

- Weih, L. R., Most, E. R., \& Rezzolla, L., Optimal Neutron-star Mass Ranges to Constrain the Equation of State of Nuclear Matter with Electromagnetic and Gravitational-wave Observations, Astrophys J., 881, 73 (2019).

LRW performed the complete data analysis, derived all results, and prepared all figures. LRW wrote the manuscript with input from LR and ERM.

Chapter 4

- Weih, L. R., Hanauske, M., \& Rezzolla, L., Postmerger Gravitational-Wave Signatures of Phase Transitions in Binary Mergers, Phys. Rev. Lett., 124, 171103 (2020).

LRW performed all simulations for this study with help from MH. LRW derived all results from these simulations and prepared all figures with input from LR and MH. LRW wrote the manuscript with input from LR and MH.

- Hanauske, M., Weih, L. R., Stöcker, H., \& Rezzolla, L., Metastable hypermassive hybrid stars as neutron-star merger remnants, European Physical Journal, in press (2021).

LRW performed all simulations. LRW prepared all figures based on ideas and with data provided by MH and with input from LR and HS. LRW contributed to all stages of writing of the manuscript. 
Chapter 5

- Weih, L. R., Olivares, H., \& Rezzolla, L., Two-moment scheme for generalrelativistic radiation hydrodynamics: a systematic description and new applications, Mon. Not. R. Astron. Soc., 495, 2285 (2020).

LRW developed the radiative transport code FRAC. With help of HO, LRW coupled the code with the GRMHD code BHAC. LRW performed all simulations and derived all results from these simulations. Furthermore, LRW wrote the manuscript with input from LR and HO.

- Weih, L. R., Gabbana, A., Simeoni, D., Rezzolla, L., Succi, S., \& Tripiccione, R., Beyond moments: relativistic lattice Boltzmann methods for radiative transport in computational astrophysics, Mon. Not. R. Astron. Soc., 498, 3374 (2020).

LRW and AG initiated this study. LRW derived this method together with AG and with input from the other authors. The implementation of this method is based on the code written by AG, to which LRW contributed. LRW performed all M1 simulations. LRW also performed some of the LB simulations. LRW coupled the code to BHAC and performed all simulations of the relativistic jets. LRW prepared most of the figures. LRW and AG wrote the manuscript with input from DS and the other authors.

Note that this Thesis is a complete monographic rewrite of the above publications. Figures have been reprinted or adapted and are marked as such.

Before these three chapters, the Thesis begins with a brief introduction of the basic equations in Chap. 2. This covers all equations and concepts that will be used throughout the Thesis, i.e., numerical relativity, general-relativistic hydrodynamics, and radiative transport. Unless stated explicitly, we use units where the speed of light and the gravitational constant are one, i.e., $c=G=1$. The most commonly used variables and abbreviations in this Thesis are summarized in Tab. 1.1 for easy reference. Greek indices range from 0 to 3 and Latin indices from 1 to 3 . If component notation is not used, vectors are written in boldface and matrices with a hat.

\begin{tabular}{l|l} 
DPT & delayed phase transition \\
EHT & Event Horizon Telescope collaboration \\
EOS & equation of state \\
ET & Einstein Toolkit \\
GRMHD & general-relativistic magneto-hydrodynamics \\
GRRTMHD & general-relativistic radiative transfer magneto-hydrodynamics \\
HMNS & hypermassive neutron star \\
LB & Lattice Boltzmann \\
LRS & left-hand side \\
LVC & LIGO and Virgo collaboration \\
M1 & Two-moment method for radiative transport \\
NICER & Neutron Star Interior Explorer \\
NPT & no phase transition \\
PPT & prompt phase transition \\
PT & phase transition \\
PTTC & phase-transition triggered collapse
\end{tabular}




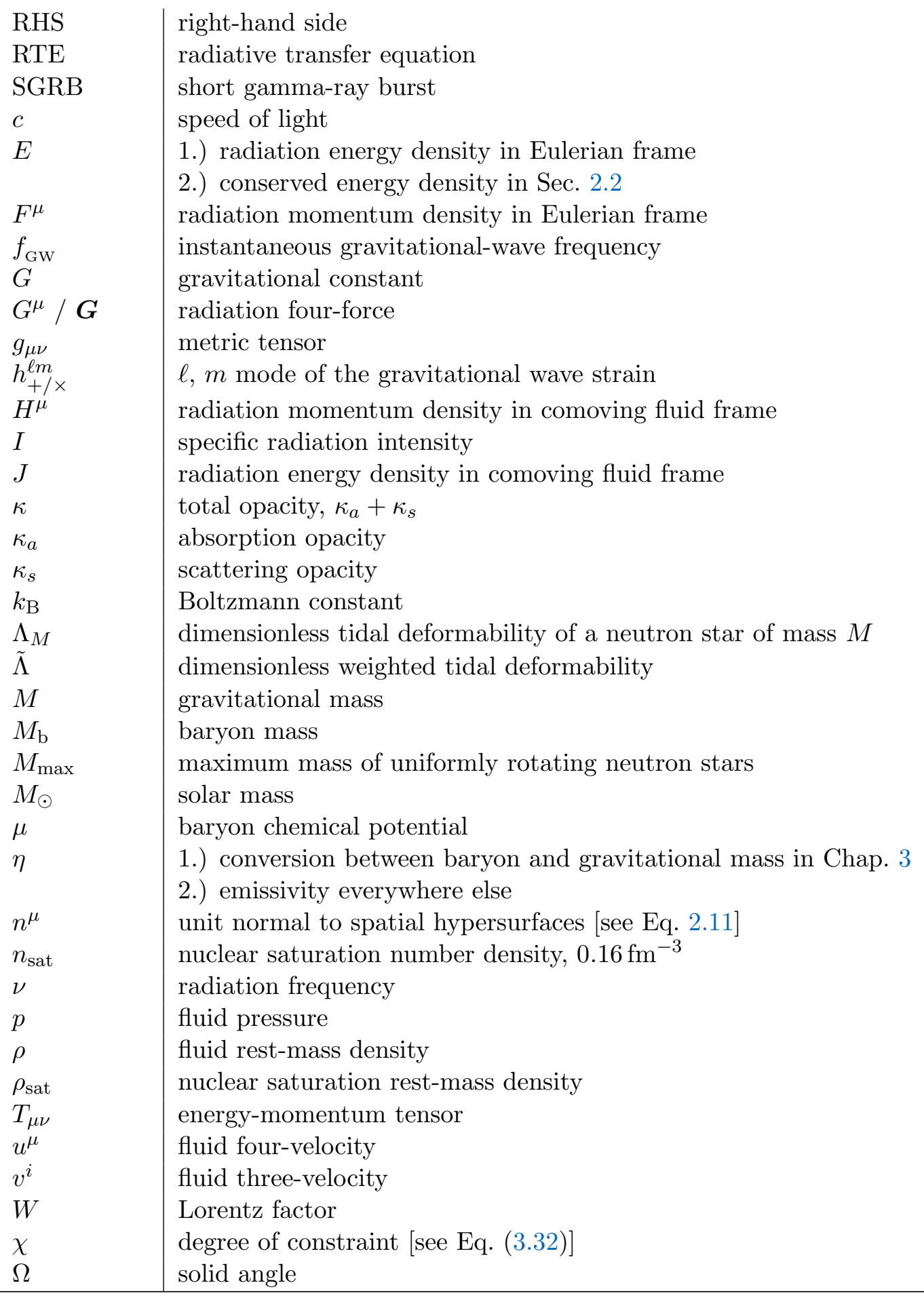

TABLE 1.1: List of commonly used abbreviations and variables throughout this Thesis. 


\section{uations \\ Basic Equations}

Exploring high-energy astrophysical phenomena via simulations requires the numerical solution of many complex equations. Especially the merging of two neutron stars includes processes across numerous scales. For a complete treatment, all four forces of nature have to be taken into account; the strong interaction governing the behavior of the matter under extreme conditions, the weak interaction responsible for neutrino radiation, electromagnetism describing the electromagnetic fields that interact with the fluid, and gravity as described by the theory of general relativity.

For the sake of completeness, and in order to allow the reader to fully understand the results presented in Chaps. 3-5, this chapter summarizes the basic equations and introduces the key methods for solving them numerically. This summary is restricted to the equations necessary within the remainder of this Thesis and in each section a number of references is given for a more comprehensive account.

\subsection{General Relativity}

In the presence of extremely compact objects, like neutron stars or black holes, classical Newtonian gravity is no longer valid. Instead, Einstein's theory of general relativity provides the governing equations. In this section a brief derivation of these field equations is presented, before summarizing the developments that led to their successful solution on the computer. A detailed introduction to general relativity can be found in Weinberg (1972); Misner et al. (1973); Carroll (2004) and to numerical relativity in Alcubierre (2008); Baumgarte \& Shapiro (2010); Shibata (2016).

\subsubsection{The Einstein Field Equations}

Within Einstein's theory of general relativity, space and time are unified within the concept of a four-dimensional manifold called spacetime. Gravity is then the consequence of the spacetime's geometry, which is determined by the distribution of the energy contained within the system under consideration. In turn, this geometry governs the motion of particles through the spacetime.

Following the principle of equivalence, the geometry is locally always Lorentzian, i.e., flat, but can otherwise be arbitrary. This condition is satisfied by the mathematical framework of Riemannian geometry. Furthermore, the principle of covariance requires the laws of physics to be form-invariant under arbitrary coordinate transformations. This can be guaranteed by writing the equations in covariant tensor form. A tensor is an algebraic object that under an arbitrary change of coordinates, 
$\left\{x_{\mu}\right\} \rightarrow\left\{\tilde{x}_{\mu}\right\}$, transforms as

$$
T_{\nu_{1} \ldots \nu_{n}}^{\mu_{1} \ldots \mu_{m}} \rightarrow \tilde{T}_{\beta_{1} \ldots \beta_{n}}^{\alpha_{1} \ldots \alpha_{m}}=\frac{\partial \tilde{x}^{\alpha_{1}}}{\partial x^{\mu_{1}}} \ldots \frac{\partial \tilde{x}^{\alpha_{m}}}{\partial x^{\mu_{m}}} \frac{\partial x^{\nu_{1}}}{\partial \tilde{x}^{\beta_{1}}} \ldots \frac{\partial x^{\nu_{n}}}{\partial \tilde{x}^{\beta_{n}}} \ldots T_{\nu_{1} \ldots \nu_{n}}^{\mu_{1} \ldots \mu_{m}},
$$

where summation over repeated indices is implied. The number of indices determines the tensor rank. A tensor of rank zero is a scalar, while a tensor of rank 1 with an upper (lower) index is called contravariant (covariant) vector. The most important rank-2 tensor is the metric $g_{\mu \nu}$, which describes the shape of the spacetime.

The ordinary partial derivative acting on a vector $A^{\nu}$ is not covariant, i.e., the expression $\partial_{\mu} A^{\nu}$ does not transform according to Eq. (2.1). Its tensor equivalent, called covariant derivative, is defined as

$$
\nabla_{\mu} A^{\nu}:=\partial_{\mu} A^{\mu}+\Gamma_{\mu \lambda}^{\nu} A^{\lambda},
$$

where the Christoffel symbols are given by

$$
\Gamma_{\beta \gamma}^{\alpha}=\frac{1}{2} g^{\alpha \delta}\left(\partial_{\gamma} g_{\delta \beta}+\partial_{\beta} g_{\delta \gamma}-\partial_{\delta} g_{\beta \gamma}\right) .
$$

This covariant derivative provides a way to measure the change of a vector when transported in a certain direction on a curved surface. It is then evident that the commutator of the covariant derivative - in contrast to that of the ordinary partial derivative - is non-zero, if the spacetime is indeed curved. Consequently, the Riemann curvature tensor, $R_{\beta \gamma \delta}^{\alpha}$, defined via

$$
2 \nabla_{[\delta} \nabla_{\gamma]} A_{\beta}:=\nabla_{\delta} \nabla_{\gamma} A_{\beta}-\nabla_{\gamma} \nabla_{\delta} A_{\beta}=: R_{\beta \gamma \delta}^{\alpha} A_{\alpha},
$$

encodes the information about the curvature of the spacetime. From Eqs. (2.2) and (2.3) its exact form follows as

$$
R_{\beta \gamma \delta}^{\alpha}=\partial_{\gamma} \Gamma_{\beta \delta}^{\alpha}-\partial_{\delta} \Gamma_{\beta \gamma}^{\alpha}+\Gamma_{\mu \gamma}^{\alpha} \Gamma_{\beta \delta}^{\mu}-\Gamma_{\mu \delta}^{\alpha} \Gamma_{\beta \gamma}^{\mu} .
$$

Contracting the first with the third index, which is the only non-trivial contraction possible for the Riemann tensor, yields the Ricci tensor, i.e.,

$$
R_{\alpha \beta}:=R_{\alpha \gamma \beta}^{\gamma}
$$

which in turn can be contracted resulting in the Ricci Scalar

$$
R:=g^{\alpha \beta} R_{\beta \alpha}=R_{\alpha}^{\alpha} .
$$

The fact that the Riemann tensor and its contractions contain information about the exact curvature of the spacetime makes them ideal candidates for the final field equations for describing gravity as the consequence of this curvature. Furthermore, these equations should satisfy the following criteria as stated in Adler et al. (1975):

1.) The field equations describing gravitation should be tensor equations.

2.) In analogy to Laplace's equation for the gravitational potential in classical mechanics, the field equations should be differential equations of second order in the components of the metric tensor.

3.) In order to guarantee a unique solution to the field equations they should be quasi-linear.

4.) In an empty space the metric must be Lorentzian. 
Since the Ricci tensor fulfills all these points, the field equations in vacuum can simply be written as

$$
R_{\mu \nu}=0 .
$$

For non-empty space, the curvature has to be related to the spacetime's energy content. This energy content is encoded in the energy-momentum tensor $T^{\mu \nu}$, which for now is an arbitrary tensor whose precise shape depends on the system under consideration (see Sec. 2.2 for how $T^{\mu \nu}$ looks like for a perfect fluid). Since the energymomentum tensor is divergence-free (reflecting the fundamental laws of conservation of energy and momentum), the Ricci tensor, whose divergence is non-vanishing, is no more suitable for the final field equations. A tensor that is constructed from the metric, fulfills the criteria 1.) - 4.) and has zero divergence is the Einstein tensor defined as ${ }^{1}$

$$
G_{\mu \nu}:=R_{\mu \nu}-\frac{1}{2} g_{\mu \nu} R
$$

While there are many other options, the Einstein tensor is the simplest one. The famous Einstein equation then follows as

$$
G_{\mu \nu}=\kappa T_{\mu \nu}
$$

where the constant $\kappa=8 \pi$ is fixed by requiring the equation to coincide with Newton's classical equation of gravity in the limit of a weak gravitational field.

Eq. (2.10) comprises six independent quasi-linear partial differential equations of second order in the metric $g_{\mu \nu}$. Only few analytic solutions of these equations are known; the most prominent one is that of the Schwarzschild metric (Schwarzschild, 1916) describing the spacetime around a point-like mass, generally interpreted as a non-rotating black hole. For more complex configurations, especially for a dynamically changing spacetime, a solution can only be obtained numerically, which will be subject of the following section.

\subsubsection{Numerical Relativity}

The time-dependent solution of a dynamically evolving system like that of a binary neutron star can be obtained numerically following the same principle as used in Newtonian mechanics: given a system's initial position and velocity at an initial time, the system's state for all future times can be computed by integrating the equations of motion forward in time. This is the traditional Cauchy problem. Likewise, in general relativity a system is initially specified by the metric $g_{\mu \nu}$ and its time derivative $\partial_{t} g_{\mu \nu}$. Specifying these quantities everywhere on a three-dimensional hypersurface, i.e., a spacelike three-surface at a constant time $t$, allows to compute the metric at any time by integrating equations for $\partial_{t}^{2} g_{\mu \nu}$, which follow from the Einstein equations.

\section{The $3+1$ Decomposition and the ADM Equations}

In general relativity the distinction between space and time is not as straightforward as it is in classical mechanics. Indeed, the interpretation of what is space and time is just the manifestation of the arbitrarily chosen set of coordinates and will be completely different in any other coordinate system. Nevertheless, in order to solve an initialvalue problem and integrate it forward in time a distinction between space and time

\footnotetext{
${ }^{1}$ An additional term including the cosmological constant is neglected here.
} 
is necessary. For this purpose, the four-dimensional spacetime is sliced into threedimensional hypersurfaces. These non-intersecting hypersurfaces are purely spatial and parameterized by a global time coordinate $t$. In this way, a time coordinate is separated from the spatial coordinates, which is known as the $3+1$ decomposition, leading to a reformulation of the Einstein equations.

First, a unit normal to these slices is defined as

$$
n_{\mu}=\alpha \nabla_{\mu} t
$$

where $\alpha$ emerges simply as a normalization constant. This constant can be computed from the normalization condition $n_{\mu} n^{\mu}=g^{\mu \nu} n_{\mu} n_{\nu}=-1$ and the fact that $n_{\mu}$ is timelike. It follows that

$$
\alpha^{2}=-\frac{1}{g^{t t}} \text {. }
$$

This constant, known as the lapse, measures the proper time that elapses between neighboring slices at times $t$ and $t+d t$. With this unit normal, the purely spatial metric that characterizes each hypersurface can then be constructed as

$$
\gamma_{\mu \nu}=g_{\mu \nu}+n_{\mu} n_{\nu}
$$

Just like $g_{\mu \nu}$ and its inverse are used to lower and raise indices of four-dimensional tensors, the three-metric, $\gamma_{\mu \nu}$, and its inverse are used to lower and raise indices of purely spatial tensors living on the three-dimensional hypersurface. Additionally, it can be used as a projection operator that projects a four-dimensional tensor onto the hypersurface. In this way, the covariant derivative defined in Eq. (2.2) can be projected in order to yield its purely spatial counterpart, i.e.,

$$
D_{\mu}:=\gamma_{\mu}^{\nu} \nabla_{\nu}
$$

which can be expressed analogously to Eq. (2.3) in terms of the purely spatial Christoffel symbols

$$
{ }^{(3)} \Gamma_{\beta \gamma}^{\alpha}=\frac{1}{2} \gamma^{\alpha \delta}\left(\partial_{\gamma} \gamma_{\delta \beta}+\partial_{\beta} \gamma_{\delta \gamma}-\partial_{\delta} \gamma_{\beta \gamma}\right) \text {. }
$$

The derivation of the spatial Riemann tensor, ${ }^{(3)} R_{\beta \gamma \delta}^{\alpha}$, Ricci tensor, ${ }^{(3)} R_{\alpha \beta}$, and Ricci scalar, ${ }^{(3)} R$, where the superscript (3) distinguishes the purely spatial quantities from their four-dimensional counterparts, is then the same as in Sec. 2.1.1, but using Eqs. (2.14) and (2.15) instead of Eqs. (2.2) and (2.3).

These purely spatial quantities are necessary in order to rewrite the Einstein field equations in a form where time and space are separated. However, these spatial quantities alone are not enough, which is evident from the fact that $R_{\beta \gamma \delta}^{\alpha}$ contains also time derivatives, while ${ }^{(3)} R_{\beta \gamma \delta}^{\alpha}$ does not. This information is instead encoded in the extrinsic curvature, which is obtained via parallel transporting the normal vector, $n_{\mu}$, along the hypersurface and then projecting its change onto this hypersurface, i.e.,

$$
K_{\mu \nu}:=-\gamma_{\mu}^{\alpha} \gamma_{\nu}^{\beta} \nabla_{\alpha} n_{\beta} .
$$

The Riemann tensor can now be projected onto the spatial direction using $\gamma_{\nu}^{\mu}$ and in the temporal direction using $n^{\mu}$. The only non-vanishing projections yield the equations of Gauss, Codazzi and Ricci, whose rather lengthy derivation is skipped here, but can be found, e.g., in Baumgarte \& Shapiro (2010). These equations can then be used to finally decompose the Einstein equations. The purely temporal contraction 
of Eq. (2.10) yields the Hamiltonian constraint equation

$$
{ }^{(3)} R+K^{2}-K_{\mu \nu} K^{\mu \nu}=16 \pi E,
$$

where $K=K_{\mu}^{\mu}$ is the trace of the extrinsic curvature and $E:=n^{\mu} n^{\nu} T_{\mu \nu}$ the temporal projection of the energy-momentum tensor and is therfore the energy density of the spacetime. The mixed temporal-spatial projection yields the momentum constraint equations

$$
D_{\nu} K_{\mu}^{\nu}-D_{\mu} K=8 \pi S_{\mu}
$$

with $S_{\mu}:=\gamma_{\mu}^{\lambda} n^{\nu} T_{\lambda \nu}$.

Evidently, Eqs. (2.17) and (2.18) do not contain any time derivatives and are written in terms of quantities that are completely specified on a given hypersurface. These equations do therefore not provide any information about how the metric changes in time, but rather provide constraints that the metric has to satisfy on every hypersurface during the time evolution. A solution of these elliptic partial differential equations can thus be used as initial data for starting a simulation. In practice, these equations are also computed during the simulation in order to monitor the quality of the evolved solution.

For deriving the evolution equations, the introduction of another quantity, i.e., the shift vector $\beta^{\mu}$, is necessary. This is because evolving vectors from a hypersurface at time $t$ to the neighboring one at time $t+d t$ along the direction $n^{\mu} d t$ will cause vectors from different points to end up on different hypersurfaces since coordinates will also change during this evolution. A unit vector taking this shift into account can be defined as

$$
t^{\mu}:=\alpha n^{\mu}+\beta^{\mu},
$$

which is dual to $\nabla_{\mu} t$. The purely spatial shift of coordinates is now incorporated in the purely spatial shift vector $\beta^{\mu}$. The four-dimensional metric, $g_{\mu \nu}$, can now be decomposed in terms of $\alpha, \beta^{i}$, and $\gamma_{\mu \nu}$, i.e., the line element in the $3+1$ decomposition reads

$$
d s^{2}=-\left(\alpha^{2}-\beta_{i} \beta^{i}\right) d t^{2}+2 \beta_{i} d x^{i} d t+\gamma_{i j} d x^{i} d x^{j} .
$$

The evolution equations for $K_{\mu \nu}$ then follow from the Ricci equation, where the Lie-derivative along $n^{\mu}$ is expressed in terms of the one along $t^{\mu}$, which ensures that after a timestep all vectors will end up on the same hypersurface. After some algebra follows

$$
\begin{aligned}
\partial_{t} K_{i j}= & -D_{i} D_{j} \alpha+\beta^{k} \partial_{k} K_{i j}+K_{i k} \partial_{j} \beta^{k}+K_{k j} \partial_{i} \beta^{k} \\
& +\alpha\left({ }^{(3)} R_{i j}+K K_{i j}-2 K_{i k} K_{j}^{k}\right)-8 \pi \alpha\left(S_{i j}-\frac{1}{2}(S-E)\right)
\end{aligned}
$$

with $S_{\mu \nu}:=\gamma_{\mu}^{\alpha} \gamma_{\nu}^{\beta} T_{\alpha \beta}$ and $S_{\mu}:=S_{\mu}^{\mu}$.

The evolution equation for the three-metric follows from the interpretation of the extrinsic curvature as the rate of change of $\gamma_{\mu \nu}$ along the normal direction. Following this interpretation, the extrinsic curvature can be defined in terms of the Lie-derivative of $\gamma_{\mu \nu}$ along $n^{\mu}$. Again, writing this Lie-derivative in terms of the one along $t^{\mu}$ and using some algebra yields the evolution equation for the three-metric, i.e.,

$$
\partial_{t} \gamma_{i j}=-2 \alpha K_{i j}+D_{i} \beta_{j}+D_{j} \beta_{i} .
$$

It is now possible to use Eqs. (2.21) and (2.22) for evolving an initial configuration for $\gamma_{i j}$ and $K_{i j}$ in time. The set of equations (2.17), (2.18), (2.21), (2.22) are referred 
to as Arnowitt-Deser-Misner (ADM) equations (Arnowitt et al., 2008). Numerically, however, solving this set of equations leads to an unstable evolution of the metric, since the equations are only weakly instead of strongly hyperbolic (see Alcubierre, 2008, for more information on hyperbolicity). A strongly hyperbolic system is guaranteed to be well-posed, i.e., its solution will grow at most exponentially (see Hilditch, 2013, for more details on hyperbolicity and well-posedness). In the ADM system, the strong hyperbolicity is spoiled by the mixed spatial derivatives hidden in the Ricci tensor ${ }^{(3)} R_{i j}$. It is therefore desirable to reformulate the equations in a way that makes them strongly hyperbolic.

\section{The BSSNOK, CCZ4 and Z4c Formulation}

The first such reformulation was developed by Baumgarte, Shapiro, Shibata, Nakamura, Ohara and Kojima (Nakamura et al., 1987; Shibata \& Nakamura, 1995; Baumgarte \& Shapiro, 1999), hence BSSNOK formulation. The main idea is to remove the mixed spatial derivatives via a conformal traceless transformation leading to new independent variables that have to be evolved. This procedure has a direct analogue in electrodynamics, where Maxwell's equations can be reformulated in the same way (Knapp et al., 2002), which due to its simplicity is often used for illustrating the procedure. Here we simply state the final system of evolution equations

$$
\begin{aligned}
\partial_{t} \tilde{\gamma}_{i j}= & -2 \alpha \tilde{A}_{i j}+2 \tilde{\gamma}_{k i} \partial_{j)} \beta^{k}-\frac{2}{3} \tilde{\gamma}_{i j} \partial_{k} \beta^{k}+\beta^{k} \partial_{k} \tilde{\gamma}_{i j} \\
\partial_{t} \tilde{A}_{i j}= & \phi^{2}\left[-D_{i} D_{j} \alpha+\alpha\left({ }^{(3)} R_{i j}-8 \pi S_{i j}\right)\right]^{\mathrm{TF}}+\beta^{k} \partial_{k} \tilde{A}_{i j}+2 \tilde{A}_{k(i} \partial_{j)} \beta^{k} \\
& +\alpha\left(\tilde{A}_{i j} K-2 \tilde{A}_{i k} \tilde{A}_{j}^{k}\right)-\frac{2}{3} \tilde{A}_{i j} \partial_{k} \beta^{k} \\
\partial_{t} \phi= & \frac{1}{3} \phi \alpha K-\frac{1}{3} \phi \partial_{i} \beta^{i}+\beta^{k} \partial_{k} \phi \\
\partial_{t} K= & -D_{i} D^{i} \alpha+\alpha\left[\tilde{A}_{i j} \tilde{A}^{i j}+\frac{1}{3} K^{2}+4 \pi(E+S)\right]+\beta^{i} \partial_{i} K \\
\partial_{t} \tilde{\Gamma}^{i}= & \tilde{\gamma}^{j k} \partial_{j} \partial_{k} \beta^{i}+\frac{1}{3} \tilde{\gamma}^{i k} \partial_{k} \partial_{j} \beta^{j}+\frac{2}{3} \tilde{\Gamma}^{i} \partial_{j} \beta^{j}-\tilde{\Gamma}^{j} \partial_{j} \beta^{i}-2 \tilde{A}^{i j} \partial_{j} \alpha+\beta^{j} \partial_{j} \tilde{\Gamma}^{i} \\
& +2 \alpha\left(\tilde{\Gamma}_{j k}^{i} \tilde{A}^{i j}-3 \tilde{A}^{i j} \partial_{j} \ln \phi-\frac{2}{3} \tilde{\gamma}^{i j} \partial_{j} K\right)-16 \pi \alpha \tilde{\gamma}^{i j} S_{j},
\end{aligned}
$$

where the superscript TF denotes the trace-free part of a tensor, $\phi:=\left[\operatorname{det}\left(\gamma_{i j}\right)\right]^{-1 / 6}$ is the conformal factor, $A_{i j}$ the trace-free extrinsic curvature, a tilde denotes the conformally-related quantities, and $\tilde{\Gamma}^{i}:=\tilde{\gamma}^{j k} \tilde{\Gamma}_{j k}^{i}$. Note that the constraint equations (2.17) and (2.18) remain unchanged.

The BSSNOK system is strongly hyperbolic and thus allows for stable numerical simulations. Indeed, it has been the standard choice for many simulations and is still widely used. However, especially in the regime of strong gravitational fields, numerical errors due to the discretization of the equations do accumulate, since the BSSNOK formulation does not allow for a damping and/or propagation of these constraint violations (Zlochower et al., 2016). This is shown in Fig. 2.1, where the Hamiltonian constraint from an exemplary two-puncture simulation (Ansorg et al., 2004 ) is monitored. Besides the grid effects at the boundaries between coarse and fine grids, the Hamiltonian constraint, $H$, is violated the most around the central black holes, where the gradients of the gravitational field are the strongest. This constraint violation grows over the course of this simulation, eventually causing it to crash. 


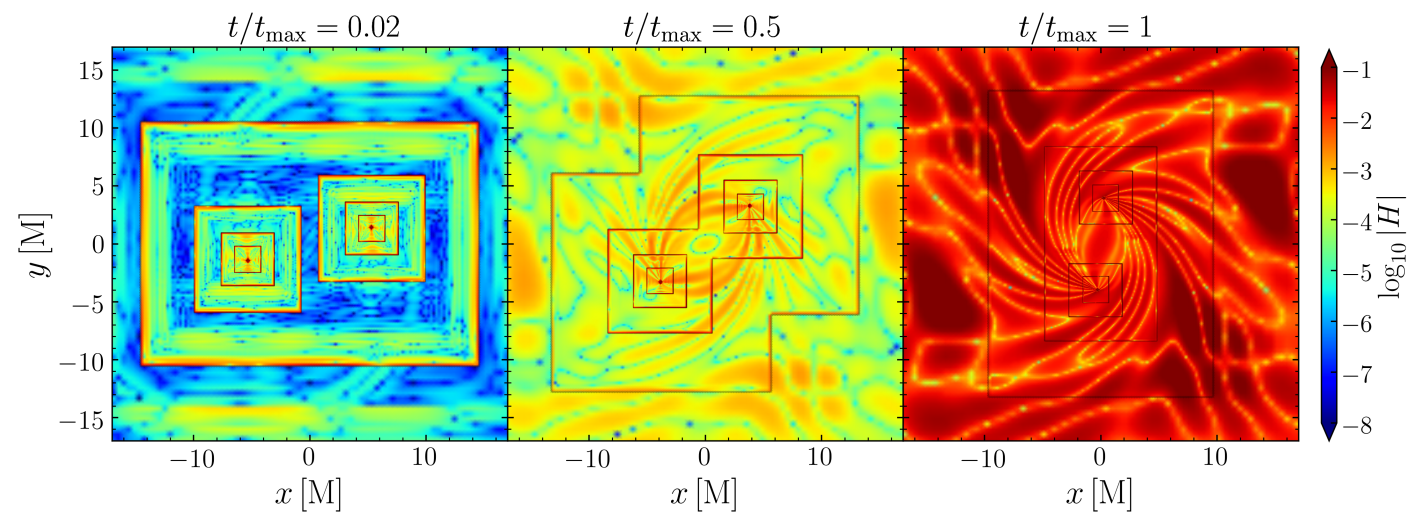

FIgURE 2.1: Hamiltonian constraint violation for a two-puncture simulation using the BSSNOK formulation. The grid contains different levels of refinement and its length is measured in terms of the initial black hole's ADM mass, $M$.

This problem can be avoided within a constraint-damping formulation, i.e., a system of equations that allows the constraints to propagate and be damped and eventually simply leave the grid. Two commonly used formulations that provide this desirable characteristic are the conformally and covariant $Z_{4}\left(C C Z_{4}\right)$ and the $Z 4 \mathrm{c}$ formulations, both variants of the Z4 system (Bona et al., 2003). The main idea of the $\mathrm{Z} 4$ system is to extend the Einstein equations by the addition of terms that algebraically evaluate to zero. This is achieved via the introduction of a new fourvector $Z_{\mu}$. The $\mathrm{Z} 4$ formulation is then written as

$$
R_{\mu \nu}+2 \nabla_{(\mu} Z_{\nu)}-\left(1+\kappa_{2}\right) g_{\mu \nu} n_{\lambda} Z^{\lambda}=8 \pi\left(T_{\mu \nu}-\frac{1}{2} g_{\mu \nu} T_{\mu}^{\mu}\right)
$$

which obviously reduces to the known Einstein equations for $Z_{\mu}=0$. A non-zero $Z_{\mu}$ measures the deviation of the numerical solution from a true solution of the Einstein equations. The advantage is now that evolving this Z-vector allows one to avoid an accumulation of the constraint violations. This happens in two ways: first, the free parameters $\kappa_{1}>0$ and $\kappa_{2}>-1$ (Gundlach et al., 2005) can be used to control the exponential decay time and secondly, making use of the Bianchi identity, a wavelike character for the evolution of $Z_{\mu}$ is revealed allowing constraint violations to propagate and leave the computational domain.

Applying the $3+1$ split to Eq. (2.28) and following the same recipe as for the BSSNOK formulation yields the CCZ4 system (Alic et al., 2012a)

$$
\begin{aligned}
\partial_{t} \tilde{\gamma}_{i j}= & -2 \alpha \tilde{A}_{i j}+2 \tilde{\gamma}_{k(i} \partial_{j)} \beta^{k}-\frac{2}{3} \tilde{\gamma}_{i j} \partial_{k} \beta^{k}+\beta^{k} \partial_{k} \tilde{\gamma}_{i j}, \\
\partial_{t} \tilde{A}_{i j}= & \phi^{2}\left[-D_{i} D_{j} \alpha+\alpha\left({ }^{(3)} R_{i j}+D_{i} Z_{j}+D_{j} Z_{i}-8 \pi S_{i j}\right)\right]^{\mathrm{TF}}+\beta^{k} \partial_{k} \tilde{A}_{i j} \\
& +2 \tilde{A}_{k(i} \partial_{j)} \beta^{k}+\alpha\left(\tilde{A}_{i j} \mathcal{K}-2 \tilde{A}_{i k} \tilde{A}_{j}^{k}\right)-\frac{2}{3} \tilde{A}_{i j} \partial_{k} \beta^{k}, \\
\partial_{t} \phi= & \frac{1}{3} \phi \alpha \mathcal{K}-\frac{1}{3} \phi \partial_{i} \beta^{i}+\beta^{k} \partial_{k} \phi, \\
\partial_{t} \mathcal{K}= & -D_{i} D^{i} \alpha+\alpha\left[{ }^{(3)} R+2 D_{j} Z^{j}+\mathcal{K}^{2}-2 \Theta \mathcal{K}\right]+\beta^{i} \partial_{i} \mathcal{K} \\
& -3 \alpha \kappa_{1}\left(1+\kappa_{2}\right) \Theta+4 \pi \alpha(S-3 E), \\
\partial_{t} \hat{\Gamma}^{i}= & 2 \tilde{\gamma}^{i j}\left(\alpha \partial_{j} \Theta-\Theta \partial_{j} \alpha-\frac{2}{3} \alpha \mathcal{K} Z_{j}\right)+2 \kappa_{3}\left(\frac{2}{3} \tilde{\gamma}^{i j} Z_{j} \partial_{k} \beta^{k}-\tilde{\gamma}^{j k} Z_{j} \partial_{k} \beta^{i}\right)
\end{aligned}
$$




$$
\begin{aligned}
& +\tilde{\gamma}^{j k} \partial_{j} \partial_{k} \beta^{i}+\frac{1}{3} \tilde{\gamma}^{i k} \partial_{k} \partial_{j} \beta^{j}+\frac{2}{3} \tilde{\Gamma}^{i} \partial_{j} \beta^{j}-\tilde{\Gamma}^{j} \partial_{j} \beta^{i}-2 \tilde{A}^{i j} \partial_{j} \alpha+\beta^{j} \partial_{j} \hat{\Gamma}^{i} \\
& +2 \alpha\left(\tilde{\Gamma}_{j k}^{i} \tilde{A}^{i j}-3 \tilde{A}^{i j} \partial_{j} \ln \phi-\frac{2}{3} \tilde{\gamma}^{i j} \partial_{j} \mathcal{K}\right)-16 \pi \alpha \tilde{\gamma}^{i j} S_{j}-2 \alpha \kappa_{1} \tilde{\gamma}^{i j} Z_{j} \\
\partial_{t} \Theta= & \frac{1}{2} \alpha\left({ }^{(3)} R+2 D_{j} Z^{j}-\tilde{A}_{i j} \tilde{A}^{i j}+\frac{2}{3} \mathcal{K}^{2}-2 \Theta \mathcal{K}\right)-Z^{j} \partial_{j} \alpha \\
& +\beta^{j} \partial_{j} \Theta-\alpha \kappa_{1}\left(2+\kappa_{2}\right) \Theta-8 \pi \alpha E
\end{aligned}
$$

where $\Theta:=-n_{\mu} Z^{\mu}, \hat{\Gamma}^{i}:=\tilde{\Gamma}^{i}+2 \tilde{\gamma}^{i j} Z_{j}$, and $\mathcal{K}:=K+2 \Theta$. The differences w.r.t. the BSSNOK system [cf., Eqs. (2.23)-(2.27)] are highlighted in red. Note that the trace of the extrinsic curvature for the CCZ4 system is different to the one of the BSSNOK system. When subtracting two times Eq. (2.34) from Eq. (2.32) according to the definition of $\mathcal{K}$, the BSSNOK equations are recovered for $\Theta=Z_{i}=0$. Note also, that the constraint violations, i.e., the components of the Z-vector, are now evolved in Eqs. (2.33) (spatial components) and (2.34) (temporal component). Furthermore, in Eq. (2.33) an additional term proportional to the constant $\kappa_{3}$ has been added. This term breaks the covariance of the CCZ4 system for $\kappa_{3} \neq 1$. It might be beneficial to sacrifice covariance for a more stable evolution, i.e., choosing $\kappa_{3}=0.5$ (Alic et al., 2012a, 2013).

Another conformal formulation of the Z4 system is the Z4c formulation (Bernuzzi \& Hilditch, 2010; Hilditch et al., 2013), where in contrast to the CCZ4 system nondamping non-primary additions to the ADM equations are dropped somewhat arbitrarily but aiming at a system closer to BSSNOK. This results in the following non-covariant set of equations

$$
\begin{aligned}
\partial_{t} \tilde{\gamma}_{i j}= & -2 \alpha \tilde{A}_{i j}+2 \tilde{\gamma}_{k(i} \partial_{j} \beta^{k}-\frac{2}{3} \tilde{\gamma}_{i j} \partial_{k} \beta^{k}+\beta^{k} \partial_{k} \tilde{\gamma}_{i j} \\
\partial_{t} \tilde{A}_{i j}= & \phi^{2}\left[-D_{i} D_{j} \alpha+\alpha\left({ }^{(3)} R_{i j}+D_{i} Z_{j}+D_{j} Z_{i}-8 \pi S_{i j}\right)\right]^{\mathrm{TF}}+\beta^{k} \partial_{k} \tilde{A}_{i j} \\
& +2 \tilde{A}_{k(i} \partial_{j} \beta^{k}+\alpha\left(\tilde{A}_{i j}(\mathcal{K}-2 \Theta)-2 \tilde{A}_{i k} \tilde{A}_{j}^{k}\right)-\frac{2}{3} \tilde{A}_{i j} \partial_{k} \beta^{k}, \\
\partial_{t} \phi= & \frac{1}{3} \phi \alpha(\mathcal{K}-2 \Theta)-\frac{1}{3} \phi \partial_{i} \beta^{i}+\beta^{k} \partial_{k} \phi \\
\partial_{t} K= & -D_{i} D^{i} \alpha+\alpha\left[\frac{(3) R+2 D_{j} Z^{j}}{}+\tilde{A}_{i j} \tilde{A}^{i j}+\frac{1}{3} \mathcal{K}^{2}=2 \Theta \mathcal{K}\right]+\beta^{i} \partial_{i} K \\
& +\alpha \kappa_{1}\left(1-\kappa_{2}\right) \Theta+4 \pi \alpha(S+E) \\
\partial_{t} \hat{\Gamma}^{i}= & 2 \tilde{\gamma}^{i j}\left(\alpha \partial_{j} \Theta-\Theta \partial_{j} \alpha-\frac{2}{3} \alpha \mathcal{K} Z_{j}\right)+2 \kappa_{3}\left(\frac{2}{3} \tilde{\gamma}^{i j} Z_{j} \partial_{k} \beta^{k}-\tilde{\gamma}^{j k} Z_{j} \partial_{k} \beta^{i}\right) \\
& +\tilde{\gamma}^{j k} \partial_{j} \partial_{k} \beta^{i}+\frac{1}{3} \tilde{\gamma}^{i k} \partial_{k} \partial_{j} \beta^{j}+\frac{2}{3} \tilde{\Gamma}^{i} \partial_{j} \beta^{j}-\tilde{\Gamma}^{j} \partial_{j} \beta^{i}-2 \tilde{A}^{i j} \partial_{j} \alpha+\beta^{j} \partial_{j} \hat{\Gamma}^{i} \\
& +2 \alpha\left(\tilde{\Gamma}_{j k}^{i} \tilde{A}^{i j}-3 \tilde{A}^{i j} \partial_{j} \ln \phi-\frac{2}{3} \tilde{\gamma}^{i j} \partial_{j} \mathcal{K}\right)-16 \pi \alpha \tilde{\gamma}^{i j} S_{j}-2 \alpha \kappa_{1} \tilde{\gamma}^{i j} Z_{j} \\
\partial_{t} \Theta= & \frac{1}{2} \alpha\left({ }^{(3)} R+2 D_{j} Z^{j}-\tilde{A}_{i j} \tilde{A}^{i j}+\frac{2}{3} \mathcal{K}^{2}=2 \Theta \mathcal{K}\right)=Z^{j} \partial_{j} \alpha \\
& +\beta^{j} \partial_{j} \Theta-\alpha \kappa_{1}\left(2+\kappa_{2}\right) \Theta-8 \pi \alpha E .
\end{aligned}
$$

This time, the differences to the CCZ4 system are highlighted. Besides a few terms being dropped (crossed out with red lines), the major difference - as evident from Eq. (2.38) - is that the BSSNOK trace $K$ is evolved rather than $\mathcal{K}$.

While CCZ4 - in contrast to Z4c - is a mathematically complete formulation, it is 


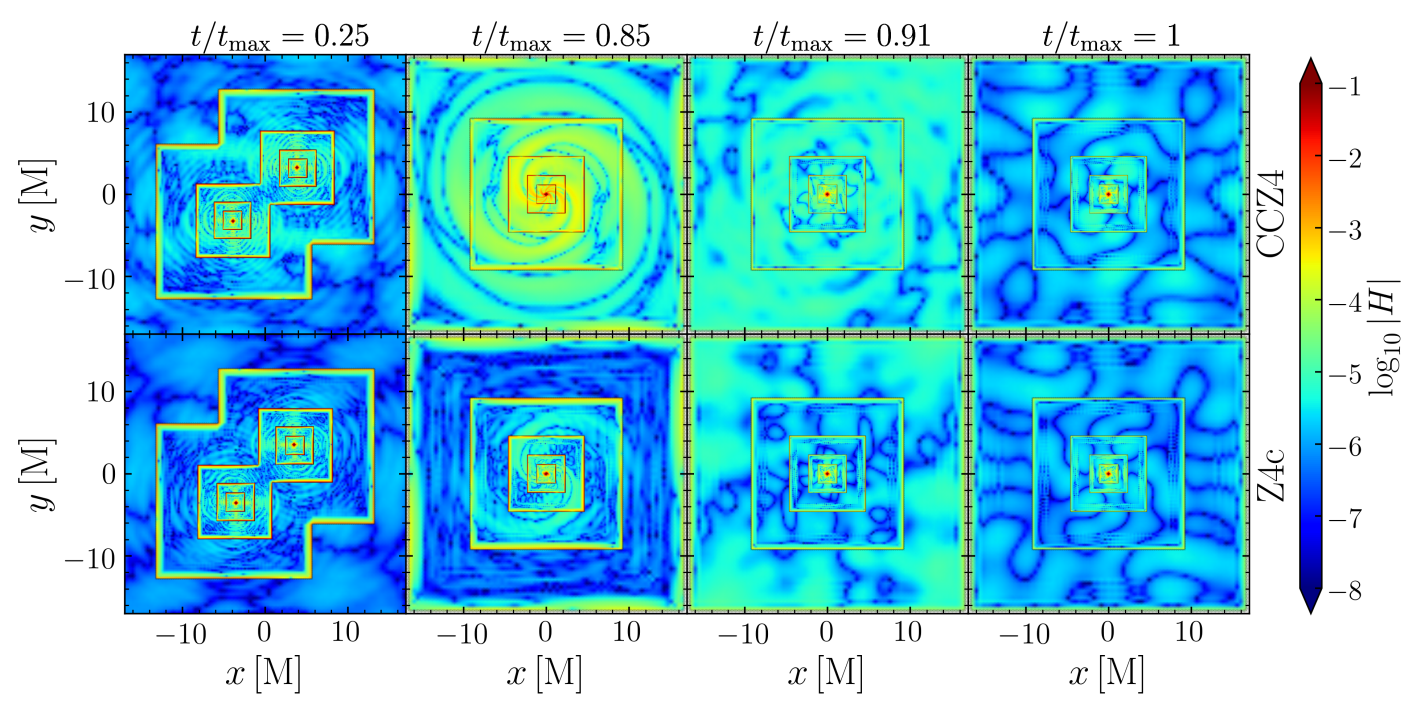

Figure 2.2: Hamiltonian constraint violation for the same two-puncture simulation as in Fig. 2.1 using the CCZ4 (top) and Z4c (bottom) formulations at four representative times.

difficult to assess, which of the above presented formulations is the best for numerical simulations. This is mostly investigated simply via trial and error as recently done in Daverio et al. (2018). In this study the BSSNOK, CCZ4 and Z4c systems are compared in the weak-field regime and the CCZ4 system was found to marginally outperform the others. This result, however, is not transferable to the strong-field regime. This regime can be tested via simulations of two-punctures. As expected, these simulations reveal that CCZ4 and Z4c perform clearly better than BSSNOK, which can be seen by comparing Fig. 2.1 with Fig. 2.2. The latter figure also shows that for both, CCZ4 and Z4c, yet still large constraint violations are produced at the point of merger (see second panels from the left). While Z4c produces smaller violations and damps these slightly faster, CCZ4 has the advantage of being a covariant formulation. Via fine-tuning parameters, especially $\kappa_{1}$, CCZ4 can achieve similarly good results as Z4c. A larger $\kappa_{1}$ generally means stronger damping and thus less constraint violations. On the other hand, since there is an inverse relation between $\kappa_{1}$ and the grid resolution, a too large value leads to overdamping and instabilities on the coarser grids. The ideal range is $\kappa_{1} \in[0.01 ; 0.05]$, while any value of $\kappa_{2} \in[-1 ; 1]$ yields equally good results and $\kappa_{3}=0.5$ has proven robust.

Apart from the mathematical considerations, it can be summarized that the question of which formulation is the better one can not be answered conclusively and in practice both have shown satisfactory results. Further fine-tuning of parameters or alternative approaches like position-dependent damping coefficients or different definitions of the evolved variables (like $K$ vs. $\mathcal{K}$ ) should be explored in future studies. Moreover, there are also different variations of both CCZ4 (Bezares et al., 2017) and Z4c (Hilditch \& Ruiz, 2018) that should also be taken into account for an extensive comparison before a definite statement about the best formulation can be made.

\section{Gauge Conditions}

Independent of which of the above formulations is used, the system of equations is not yet complete. The slicing condition for the lapse, $\alpha$, and the spatial shift condition for the shift vector, $\beta^{i}$, need to be specified. These four degrees of freedom reflect the free choice of coordinates in general relativity. In the picture of the $3+1$ decomposition 
they represent the free choice of how to slice the four-dimensional spacetime manifold and lay out coordinates on each three-dimensional hypersurface. While this choice is completely arbitrary, there are some that have proven to be more useful than others. 2008)

A commonly used gauge is the $1+\log$ slicing (Bona et al., 1997; Brügmann et al.,

$$
\partial_{t} \alpha-\beta^{i} \partial_{i} \alpha=-2 \alpha K
$$

which has the advantage to be singularity avoiding, i.e., in the vicinity of a singularity Eq. (2.41) causes the proper time to elapse slowly.

The shift condition should be chosen so that the change of the shape of a volume element is minimal when taken from one hypersurface to the next. The conditions $\partial_{t} \tilde{\Gamma}^{i}=0$ obey this (Alcubierre, 2008), but result in three elliptic equations, which are computationally expensive to solve. The Gamma-driver condition only approximates this condition by driving $\tilde{\Gamma}^{i}$ to remain approximately constant (Alcubierre et al., 2003). This results in the following set of equations

$$
\begin{aligned}
\partial_{t} \beta^{i}-\beta^{j} \partial_{j} \beta^{i} & =\frac{3}{4} B^{i}, \\
\partial_{t} B^{i}-\beta^{j} \partial_{j} B^{i} & =\partial_{t} \tilde{\Gamma}^{i}-\beta^{k} \partial_{k} \tilde{\Gamma}^{i}-\eta B^{i},
\end{aligned}
$$

with the auxiliary variable $B^{i}$. The damping constant $\eta$ is typically chosen to be of the order of the inverse mass contained within the simulated system.

\subsubsection{Gravitational Waves}

One of the main goals of simulations in numerical relativity is to compute the form of the gravitational waves emitted by the system in order to compare to the measurements of gravitational-wave detectors. Mathematically speaking, a gravitational wave arises from a small perturbation to the flat metric, i.e., the Minkowski metric $\eta_{\mu \nu}$, written as

$$
g_{\mu \nu}=\eta_{\mu \nu}+h_{\mu \nu} \quad \text { with } \quad\left|h_{\mu \nu}\right| \ll 1 .
$$

While such a perturbation could have any arbitrary form, plugging Eq. (2.44) in the Einstein equations (2.8) for a vacuum spacetime and choosing a suitable gauge, i.e., $\nabla_{\mu} h^{\mu \nu}=0, h_{\mu 0}=0$ and $h_{\mu}^{\mu}=0$, reveals that the perturbation $h_{\mu \nu}$ follows the equation of motion for a wave (Baumgarte \& Shapiro, 2010), i.e.,

$$
\square h_{\mu \nu}:=\nabla_{\lambda} \nabla^{\lambda} h_{\mu \nu}=0 \text {. }
$$

After choosing the gauge conditions for $h_{\mu \nu}$, two degrees of freedom still remain being the two possible polarizations of the gravitational wave. This is highlighted by writing $h_{\mu \nu}$ in terms of the polarization tensors $e_{\mu \nu}^{+}$and $e_{\mu \nu}^{\times}$as

$$
h_{\mu \nu}=h_{+} e_{\mu \nu}^{+}+h_{\times} e_{\mu \nu}^{\times},
$$

where $h_{+}$and $h_{\times}$are the dimensionless amplitudes of the plus- and cross-polarizations and the polarization tensors are characterized by all components being zero except for $e_{x x}^{+}=-e_{y y}^{+}=e_{x y}^{\times}=e_{y x}^{\times}=1$. The amplitudes $h_{+}$and $h_{\times}$are also called the strain amplitudes of the gravitational wave and are directly related to the relative distortion of the arms of a detector. It is therefore this strain that is measured by the gravitational-wave detectors making it one of the key quantities to be computed within numerical relativity simulations. 
Numerically, the strain is computed from the Weyl tensor within the NewmanPenrose formalism (Newman \& Penrose, 1962; Newman \& Penrose, 1963). The Weyl tensor $C_{\alpha \beta \gamma \delta}$ is the trace-free part of the Riemann tensor. Its contraction with a specific null tetrad yields five scalars. The aim is to choose the null tetrad in a way so that these scalars have a simple physical interpretation. This is achieved for a tetrad with radially in- and outgoing null vectors, i.e., $l^{\mu}$ and $k^{\mu}$, respectively, and a complex vector $m^{\mu}$ that fulfills

$$
-l^{\mu} k_{\mu}=1=m^{\mu} \bar{m}_{\mu},
$$

where $\bar{m}^{\mu}$ is the complex conjugate of $m^{\mu}$. This still leaves some freedom for the choice of $l^{\mu}, k^{\mu}$ and $m^{\mu}$ and a common choice is constructing the tetrad from orthonormal spatial vectors (see e.g., Brügmann et al., 2008, for the exact form of such a tetrad within the $3+1$ formulation). Out of the five possible scalars that can be obtained from contracting the Weyl tensor, the most important one - often simply referred to as Weyl scalar - is obtained via the following contraction

$$
\Psi_{4}:=-C_{\alpha \beta \gamma \delta} k^{\alpha} \bar{m}^{\beta} k^{\gamma} \bar{m}^{\delta}
$$

and represents a measure of the radially outgoing gravitational radiation.

Writing out Eq. (2.48) results in an expression with a complex dependency on the Riemann tensor. During a simulation, the Riemann tensor, and thus $\Psi_{4}$, can be computed from the evolved variables using again the equations of Gauss, Codazzi and Ricci (Baumgarte \& Shapiro, 2010). In post-processing the gravitational wave strain can then be computed from the Weyl scalar via the relation ${ }^{2}$

$$
\Psi_{4}=\ddot{h}_{+}-i \ddot{h}_{\times} .
$$

This requires to integrate in time twice, i.e.,

$$
h:=h_{+}-i h_{\times}=\int_{-\infty}^{t} \mathrm{~d} t^{\prime} \int_{-\infty}^{t^{\prime}} \Psi_{4} \mathrm{~d} t^{\prime \prime}
$$

where numerically the integral is best evaluated in the frequency domain (Reisswig \& Pollney, 2011).

Writing the complex-valued strain in its Eulerian representation, i.e.,

$$
h=h_{\mathrm{eff}} e^{-i \varphi}
$$

with amplitude (often referred to as effective strain) $h_{\mathrm{eff}}=\sqrt{h_{+}^{2}+h_{\times}^{2}}$ and phase angle $\varphi$ allows to derive the instantaneous gravitational wave frequency

$$
f_{\mathrm{GW}}=\dot{\varphi}=-\operatorname{Im}\left(\frac{\dot{h}}{h}\right)=-\operatorname{Im}\left(\frac{\dot{h}_{\mathrm{eff}}}{h_{\mathrm{eff}}}-i \dot{\varphi}\right) .
$$

Another important quantity useful for gravitational-wave spectroscopy (Rezzolla \& Takami, 2016) is the power spectral density

$$
\tilde{h}_{\mathrm{eff}}(f)=\sqrt{\frac{\left|\tilde{h}_{+}(f)\right|^{2}+\left|\tilde{h}_{\times}(f)\right|^{2}}{2}},
$$

\footnotetext{
${ }^{2}$ This expression holds at an infinite distance from the emitting source, which in practice requires a large computational domain typically achieved with mesh-refinement methods.
} 
derived from the Fourier transforms $\tilde{h}_{+/ \times}(f)$. The Fourier transformed effective strain is then used to compute the signal-to-noise ration (SNR) as

$$
\mathrm{SNR}=\left(\int_{0}^{\infty} \frac{\left|2 \tilde{h}_{\mathrm{eff}}(f) \sqrt{f}\right|^{2}}{\mathcal{S}(f)} \frac{\mathrm{d} f}{f}\right)^{1 / 2} .
$$

The SNR is a measure for how large the signal's amplitude per root Hertz is relative to that of the noise. For a given detector's sensitivity curve, $\mathcal{S}(f)$, it can then be evaluated whether a theoretically simulated signal could be detected by this detector (see also Cutler \& Thorne, 2002, for more information on the SNR).

Finally, it is noted that in most applications it makes sense to decompose $\Psi_{4}$ into $s=-2$ spin-weighted spherical harmonics (Gómez et al., 1997) due to symmetries in the evolved system. The Weyl scalar is then written as (Baumgarte \& Shapiro, 2010)

$$
\Psi_{4}(t, r, \theta, \phi)=\sum_{l=2}^{\infty} \sum_{m=-\ell}^{\ell} \Psi_{4}^{\ell m}(t, r) Y_{\ell m}(\theta, \phi),
$$

with expansion coefficients

$$
\Psi_{4}^{\ell m}=\int \bar{Y}_{\ell m} \Psi_{4} \mathrm{~d} \Omega
$$

For most astrophysical configurations, the $\ell=2=m$ mode is the dominant one and thus only $\Psi_{4}^{22}$ and correspondingly $h_{+/ \times / \text {eff }}^{22}$ are considered.

\subsection{General Relativistic Hydrodynamics}

The formulations of the Einstein equations presented in the previous section allow to numerically evolve the metric and the extrinsic curvature in time. In the presence of matter, solving these equations requires knowledge of the energy-momentum tensor, $T_{\mu \nu}$, which has not been specified so far and also evolves dynamically in time. The evolution of this energy-momentum tensor is governed by the laws of general relativistic hydrodynamics, which will be introduced within this section. After stating the necessary equations, a short summary of the basic numerical methods for solving them is given.

\section{The Valencia Formulation}

The evolution of the fluid, which approximates the system under consideration, is governed by the conservation laws for energy and momentum, i.e.,

$$
\nabla_{\mu} T^{\mu \nu}=0
$$

and the conservation of rest-mass density, i.e.,

$$
\nabla_{\mu}\left(\rho u^{\mu}\right)=0 \text {. }
$$

Here the fluid rest-mass density $\rho$ and its four-velocity $u^{\mu}$ is introduced. Within the $3+1$ split, the latter is written in terms of the projected three-velocity $v^{i}=\gamma_{\mu}^{i} u^{\mu} / W$, 
and the Lorentz factor $W=1 / \sqrt{1-v^{i} v_{i}}$ as (Rezzolla \& Zanotti, 2013)

$$
u^{\mu}=W\left(v^{i}-\frac{\beta^{i}}{\alpha}\right) .
$$

Together with the fluid pressure, $p$, and its total energy density, $e$, one can then specify the energy-momentum tensor as

$$
T^{\mu \nu}=(e+p) u^{\mu} u^{\nu}+p g^{\mu \nu} .
$$

Note that Eq. (2.60) is that of a perfect fluid, i.e., a fluid with vanishing viscosity and heat fluxes (see Rezzolla \& Zanotti, 2013, for more general types of fluids).

Eqs. (2.57) and (2.58) are not useful for numerical integration since they are not written in conservative form. Conservative means that the evolution equations are written in the form

$$
\partial_{t} \boldsymbol{U}+\nabla \boldsymbol{F}=\boldsymbol{S},
$$

where $\boldsymbol{U}$ is the state vector of the evolved variables, and $\boldsymbol{F}$ and $\boldsymbol{S}$ are the flux and source vector, respectively, which can depend on $\boldsymbol{U}$, but not its derivatives (Rezzolla \& Zanotti, 2013). Besides being strongly hyperbolic and thus well-posed (as are the BSSNOK, CCZ4 and Z4c equations), an equation in this form has another crucial advantage: according to the theorems by Lax \& Wendroff (1960) and Hou \& LeFloch (1994) a solution to Eq. (2.61) is guaranteed to converge to the correct solution even in the presence of discontinuities, while it is guaranteed to converge to the wrong solution if the equations are not written in conservative form and if there is a discontinuity. In contrast to the metric, which is smooth, a fluid flow is likely to develop discontinuities, physically interpreted as shocks, making the use of a conservative scheme inevitable.

Fortunately, Eq. (2.58) is already in conservative form, which is evident when expanding the covariant derivative

$$
\nabla\left(\rho u^{\mu}\right)=\frac{1}{\sqrt{-g}}\left[\partial_{t}\left(\sqrt{-g} \rho u^{0}\right)+\partial_{i}\left(\sqrt{-g} \rho u^{i}\right)\right]=0 .
$$

Eq. (2.57), on the other hand, is not in conservative form, but can be recast accordingly (Banyuls et al., 1997). Expanding the covariant derivative, using the explicit expression Eq. (2.60) for the energy-momentum tensor and identifying $S^{\mu \nu}=$ $\rho h W^{2} v^{\mu} v^{\nu}+p \gamma^{\mu \nu}$ and $S^{\mu}=\rho h W^{2} v^{\mu}$, where $h=(e+p) / \rho$ is the specific enthalpy, yields the conserved version of the general-relativistic momentum equation

$$
\partial_{t}\left(\sqrt{\gamma} S_{j}\right)+\partial_{i}\left[\sqrt{\gamma}\left(\alpha S_{j}^{i}-\beta^{i} S_{j}\right)\right]=\frac{1}{2} \sqrt{-g} T^{\mu \nu} \partial_{j} g_{\mu \nu} .
$$

The relativistic energy equation in conserved form follows similarly from a temporal projection of $T^{\mu \nu}$ as

$$
\partial_{t}(\sqrt{\gamma} E)+\partial_{i}\left[\sqrt{\gamma}\left(\alpha S^{i}-\beta^{i} E\right)\right]=-\frac{1}{2} \sqrt{-g} T^{\mu \nu} \nabla_{\mu} n_{\nu} .
$$

where $E=\rho W^{2}-p$.

To summarize, the equations of general-relativistic hydrodynamics are written within the $3+1$ decomposition in conservative form as in Eq. (2.61) with (Martí 
et al., 1991)

$$
\boldsymbol{U}=\left(\begin{array}{c}
D \\
S_{j} \\
E
\end{array}\right):=\left(\begin{array}{c}
\rho W \\
\rho h W^{2} v_{j} \\
\rho h W^{2}-p
\end{array}\right), \quad \boldsymbol{F}^{i}:=\left(\begin{array}{c}
\alpha v^{i} D-\beta^{i} D \\
\alpha S_{j}^{i}-\beta^{i} S_{j} \\
\alpha S^{i}-\beta^{i} E
\end{array}\right),
$$

and with the sources (also expanded in $3+1$ form)

$$
\boldsymbol{S}=\left(\begin{array}{c}
0 \\
\frac{1}{2} \alpha S^{i k} \partial_{j} \gamma_{i k}+S_{i} \partial_{j} \beta^{i}-E \partial_{j} \alpha \\
\alpha S^{i j} K_{i j}-S^{j} \partial_{j} \alpha
\end{array}\right)
$$

This system of equations consists of five equations for the six unknown primitive variables $\left(e, p, \rho, v^{i}\right)$. In order to close the system of equations a closure, the EOS, is required relating $p$ to $e$ and $\rho$. There are many different types of EOSs (see Sec. 2.4 in Rezzolla \& Zanotti, 2013) that will be introduced in detail throughout this Thesis in the corresponding sections, where they are needed.

Furthermore, the above set of equations can also be expanded in order to account for electromagnetic effects (see e.g., Baumgarte \& Shapiro, 2003; Del Zanna et al., 2007; Etienne et al., 2012), resulting in the equations of general relativistic magneto-hydrodynamics (GRMHD). In addition to Eqs. (2.57) and (2.58), GRMHD also includes the Maxwell equations and adds a contribution to the energy-momentum tensor, so that $T^{\mu \nu}=T_{\text {matter }}^{\mu \nu}+T_{\mathrm{EM}}^{\mu \nu}$. A derivation of the GRMHD equations and how to solve them numerically can be found in Most (2020). In the remainder of this Thesis, electromagnetic effects are not considered.

\section{Numerical Methods for General Relativistic Hydrodynamics}

Eq. (2.61) is a first-order partial differential equation with derivatives in time and space whose numerical evaluation is non-trivial. This is mostly due to the fact that the fluid variables - in contrast to the metric - are not guaranteed to be smooth during the evolution and in general will exhibit shocks. Therefore, the equation needs to be discretized using a conservative numerical method, which is either done via the finite-difference or the finite-volume approach. While in the former, the state $\boldsymbol{U}$ is represented by a point-wise representation at the underlying grid's cell-centers, the latter defines volume-averaged quantities. For a second-order accurate scheme ${ }^{3}$ both methods coincide. Since this is the most commonly implemented case (Giacomazzo \& Rezzolla, 2007; Foucart et al., 2013; Etienne et al., 2015; Porth et al., 2017) and also what will be used in this work, the discussion here is restricted to second-order schemes; details on higher-order methods can be found in Rezzolla \& Zanotti (2013) and an example for a higher-order implementation in Most et al. (2019b).

In the case of second-order accuracy, the discretized version of Eq. (2.61) for a grid with grid-spacing $\Delta x$ is written for both methods as (Rezzolla \& Zanotti, 2013)

$$
U_{i}^{n+1}=U_{i}^{n}+\frac{\Delta t}{\Delta x}\left(F_{i-1 / 2}-F_{i+1 / 2}\right)+\Delta t S_{i}
$$

where $U_{i}^{n}$ is the state at position $x=x_{i}$, i.e., at the $i$ th grid-cell, after $n$ discrete evolution steps, $F_{i \pm 1 / 2}$ is the flux at the cell interfaces, and $S_{i}$ the source term in the

\footnotetext{
3 The order measures how large the solution's error is in terms of the grid-spacing; a onedimensional grid with cells separated by $d x$ will have an error of $\mathcal{O}\left(d x^{p}\right)$ for a scheme of order $p$.
} 
$i$ th cell. Note that Eq. (2.67) is written for only one dimension for simplicity; the multi-dimensional case simply follows via adding another flux term on the RHS for each dimension and evaluating them independently.

The timestep $\Delta t$ is chosen according to the Courant-Friedrichs-Lewy (CFL) condition (Courant \& Hilbert, 1962) ensuring that during a timestep information can at most travel at the numerical speed $\lambda_{\max }:=\Delta x / \Delta t$, i.e., at most from one grid cell to its neighbor. A timestep satisfying this condition is

$$
\Delta t=\mathrm{CFL} \frac{\Delta x}{\lambda}
$$

with the CFL number being smaller than unity and $\lambda$ either set to unity or to the largest characteristic wave speed of the system (see below for the definition of the characteristic wave speed).

The fluxes on the RHS of Eq. (2.67) are computed following Godunov's insight that from the discretization of $\boldsymbol{U}$ a local Riemann problem arises between adjacent cell interfaces (Godunov, 1959). At these interfaces the discrete solution has a discontinuity characterized by the jump between the state at the left, $U_{L}^{n}$, and at the right, $U_{R}^{n}$, of the interface, i.e., at any given time $t=t_{0}$

$$
\boldsymbol{U}\left(x, t_{0}\right)= \begin{cases}\boldsymbol{U}_{L} & \text { for } x<x_{i+1 / 2}, \\ \boldsymbol{U}_{R} & \text { for } x>x_{i+1 / 2}\end{cases}
$$

The value of $\boldsymbol{U}$ at position $x_{i+1 / 2}$ is than computed by solving the Riemann problem, so that the flux $F_{i+1 / 2}$ can then be computed from $U_{i+1 / 2}$.

Originally, Godunov simply used a piece-wise constant representation of the left and right states, i.e., $\boldsymbol{U}_{L}=U_{i}$ and $\boldsymbol{U}_{R}=U_{i+1}$. More modern methods, collectively known as high-resolution shock-capturing (HRSC) methods, use sophisticated reconstruction schemes in order to compute the left and right states for the Riemann problem. While an overview of these methods is given in Toro (2009); Trangenstein (2009), we here only present the commonly used minmod slope limiter reconstruction (Kolgan, 1972; van Leer, 1979) as an example. Within this method the left and right states are piece-wise linear as opposed to the original piece-wise constant representation, i.e., the left/right state at the interface between the $i$ th and $(i+1)$ th cell is

$$
\begin{aligned}
U_{L, i} & =U_{i}+\sigma_{i}\left(x_{i+1 / 2}-x_{i}\right), \\
U_{R, i} & =U_{i+1}+\sigma_{i}\left(x_{i+1 / 2}-x_{i+1}\right),
\end{aligned}
$$

where the slope $\sigma_{i}$ is computed as

$$
\sigma_{i}=\operatorname{sign}(\alpha) \max [0, \min (|\alpha|, \beta \operatorname{sign}(\alpha))]
$$

with $\alpha:=\left(U_{i}-U_{i-1}\right) / \Delta x$ and $\beta:=\left(U_{i+1}-U_{i}\right) / \Delta x$.

Independently of the used reconstruction method, the Riemann problem is then typically solved with an approximate Riemann solver. Starting from $\boldsymbol{F}_{L}:=\boldsymbol{F}\left(\boldsymbol{U}_{L}\right)$ and $\boldsymbol{F}_{R}:=\boldsymbol{F}\left(\boldsymbol{U}_{R}\right)$. The commonly used Harten-Lax-Van Leer-Einfeldt (HLLE) solver assumes that only two waves result from the Riemann problem (Harten et al., 1983; Einfeldt, 1988). These waves propagate at the characteristic speeds $\lambda_{R}$ and $\lambda_{L}$ to the right and left, respectively, of the initial discontinuity. The state in between these 
two waves is then represented by (Rezzolla \& Zanotti, 2013)

$$
\boldsymbol{U}^{\mathrm{HLLE}}=\frac{\lambda_{R} \boldsymbol{U}_{R}-\lambda_{L} \boldsymbol{U}_{L}+\boldsymbol{F}_{L}-\boldsymbol{F}_{R}}{\lambda_{R}-\lambda_{L}} .
$$

From this equation together with the Rankine-Hugoniot conditions follows the final flux at the cell interfaces as

$$
\boldsymbol{F}^{\mathrm{HLLE}}=\frac{\lambda_{R} \boldsymbol{F}_{L}-\lambda_{L} \boldsymbol{F}_{R}+\lambda_{R} \lambda_{L}\left(\boldsymbol{U}_{R}-\boldsymbol{U}_{L}\right)}{\lambda_{R}+\lambda_{L}} .
$$

This flux is then used in Eq. (2.67) for $F_{i \pm 1 / 2}$.

To summarize, numerically Eq. (2.67) is solved using the reconstruct-solve-update scheme, which consists of the following steps:

1.) Reconstruct the state at the grid-cell's interfaces. For every cell the state $U_{i}$ can be used to reconstruct $U_{L, i}$ and $U_{R, i-1}$ using a reconstruction method like for example the above presented minmod slope limiter. Consequently, at each interface two states exist, i.e., one reconstructed from the left and one from the right.

2.) Compute the fluxes $\boldsymbol{F}_{L}$ and $\boldsymbol{F}_{R}$ and the characteristic wave speeds from the reconstructed state vector at the cell interfaces. Using an approximate Riemann solver like the HLLE-solver then yields the final fluxes at the cell interfaces.

3.) After computing $F_{i \pm 1 / 2}$ for every $i$ and the source term $S_{i}$, the state at the next timestep can be obtained via any time-integration method.

The last step, commonly referred to as the method of lines, highlights the separation between spatial and temporal derivatives in Eq. (2.61). After step 2, the equation can be reduced to a simple ordinary differential equation of the form

$$
\partial_{t} \boldsymbol{U}=\operatorname{RHS}(\boldsymbol{U}),
$$

where $\operatorname{RHS}(\boldsymbol{U})$ includes the flux terms and all other source terms. As long as all terms in $\operatorname{RHS}(\boldsymbol{U})$ are non-stiff, any explicit solver, like the commonly used explicit RungeKutta methods (Hairer et al., 1993), can be used for the final time update, where steps 1 and 2 have to be repeated for every substep. In case of stiff terms, i.e., terms, whose numerical value is much larger than that of the current state vector, an implicit method has to be used. Since usually both stiff and non-stiff terms are present, it makes sense to separate these terms, i.e., to write

$$
\partial_{t} \boldsymbol{U}=X(\boldsymbol{U})+M(\boldsymbol{U}),
$$

where $X(\boldsymbol{U})$ denotes non-stiff and $M(\boldsymbol{U})$ stiff terms. An implicit-explicit (IMEX) method can than be used as efficient time stepper (Pareschi \& Russo, 2005). Such an IMEX method will be presented in Sec. 5.1.1. In numerical astrophysics, the fluxes and gravitational source terms are absorbed in $X(\boldsymbol{U})$, while resistive magnetohydrodynamics (see e.g., Palenzuela et al., 2009; Ripperda et al., 2019) or collisional source terms due to radiative transport attribute terms to $M(\boldsymbol{U})$. The latter will be discussed exhaustively in the next section and in Chap. 5. 


\subsection{Radiative Transport}

The equations of general relativity and hydrodynamics presented above are in many cases enough for simulating high-energy astrophysical phenomena. In the case of binary neutron stars, for example, the gravitational-wave signal or the merger remnant's dynamics on time-scales of $\sim 10-100 \mathrm{~ms}$, can be modeled accurately solving these equations alone, as will be the case in Chap. 4 .

In reality, the energy-momentum conservation can also be affected by radiation in the form of neutrinos or photons. Due to the extreme conditions found in these systems huge amounts of radiation are produced; this radiation interacts with the underlying fluid and in many cases significantly alters its dynamics and composition. Whenever the interaction with the radiation can not be neglected, a proper treatment of its dynamics and its backreaction with the fluid is necessary. The description of this dynamics, called radiative transport, still poses a major challenge in numerical relativity. Why this is so and how this problem can be tackled in order to achieve a new level of realism in simulations of high-energy astrophysical phenomena is discussed in depth within this Thesis. For that purpose we first introduce the basic concepts and underlying equations of radiative transport within this section. Based on this, Chap. 5 then presents state-of-the-art radiative transfer methods and their application.

\subsubsection{The Radiative Transfer Equation}

The fundamental equation describing not only radiation, but any statistical distribution of particles out of thermodynamic equilibrium, is the Boltzmann equation. For a more intuitive understanding of this complex integro-differential equation, we start with the classical (non-relativistic) version written as (Harris, 1971)

$$
\left(\frac{\partial}{\partial t}+\frac{p^{i}}{m} \frac{\partial}{\partial x^{i}}+F^{i} \frac{\partial}{\partial p^{i}}\right) f=\mathcal{C}(f)
$$

This equation describes the change in the distribution $f\left(x^{i}, p^{i}, t\right)$ describing particles of mass $m$ and momentum $p^{i}$ at position $x^{i}$ and at time $t$. As can be easily seen, this change is due to the particle's inertial motion (second term on the LHS), their change in momentum due to an external force $F^{i}$ (third term on the LHS) and due to collisions described by the collisional integral $\mathcal{C}$. The latter can have an arbitrary form - typically including multidimensional integrals over the phase-space $d^{3} x d^{3} p$ - that depends on the underlying microphysics describing the interactions between particles. At this point, it is already evident that the seven-dimensional ${ }^{4}$ nature of the Boltzmann equation poses a major challenge for a numerical solution. Indeed, solving the equation using a seven-dimensional discretization is unfeasible even for the largest of the current supercomputers.

Radiation is generally described by massless particles, i.e., photons or neutrinos ${ }^{5}$. In this case, and when neglecting external forces, the Boltzmann equation reduces to

\footnotetext{
${ }^{4}$ Three spatial dimension + three dimensions in momentum space + one temporal dimension.

${ }^{5}$ While neutrinos are massless in the standard model of particle physics, experiments so far only provide upper bounds on the neutrino mass (Aker et al., 2019). In any case, the neutrino mass is negligibly small compared to that of hadrons and consequently assumed to be zero in numerical astrophysics.
} 
the radiative transfer equation ( $\mathrm{RTE}$ ), i.e.,

$$
\left(\frac{\partial}{\partial t}+\hat{n}^{i} \frac{\partial}{\partial x^{i}}\right) f_{(\nu)}=\mathcal{C}_{\text {rad }}\left(f_{(\nu)}\right)
$$

where $\hat{n}^{i}$ is a unit vector in the direction of propagation of the radiation particles and $f_{(\nu)}:=f\left(x^{i}, n^{i}, \nu, t\right)$, where $\nu$ (not to be confused with a covariant index) is the frequency of the particle. Note that $f_{(\nu)}$ still depends on only seven (and not eight) variables, since $\hat{n}^{i}=p^{i} / \nu^{2}$ and $\nu^{2}-\left(p_{i} p^{i}\right)^{2} \equiv 0$ according to the dispersion relation. In other words, we shift the dependence of $f$ on the three-momentum, $p^{i}$, to a dependence on the direction of propagation, $\hat{n}^{i}$, where one degree of freedom is reduced by the normalization condition for $\hat{n}^{i}$ and reappears in $\nu$.

The RTE describes the advection of radiation particles at the speed of light, whose distribution function is changed via collisional processes. These collisional processes include the emission, absorption, and scattering of particles. Consequently, the collisional source term for radiation is written as

$$
\mathcal{C}_{\text {rad }}=\tilde{\eta}_{(\nu)}-\kappa_{a,(\nu)} f_{(\nu)}+\mathcal{C}_{\text {scat }}
$$

where the emissivity $\tilde{\eta}_{(\nu)}$ and the absorption opacity $\kappa_{a,(\nu)}$ (also known as extinction coefficient) depend on the frequency and are in general determined by the properties of the underlying fluid, which emits, absorbs and scatters the radiation. The emission term is independent of the radiation's distribution and is determined by the fluid only, while the absorption term is directly proportianal to the radiation distribution, i.e., the more radiation particles are present, the more can be absorbed. The scattering term $\mathcal{C}_{\text {scat }}$ in its most general form is written as (Bruenn, 1985; Rampp, 2000; Shibata et al., 2011)

$$
\mathcal{C}_{\text {scat }}=\int_{0}^{\infty} \nu^{\prime 2} \mathrm{~d} \nu^{\prime} \int_{4 \pi} f_{\left(\nu^{\prime}\right)}^{\prime}\left(1-f_{(\nu)}\right) R^{\text {in }}-f_{(\nu)}\left(1-f_{\left(\nu^{\prime}\right)}^{\prime}\right) R^{\text {out }} \mathrm{d} \Omega^{\prime},
$$

where $R^{\text {in/out }}$ is the scattering kernel for the incoming/outgoing radiation particle and $\mathrm{d} \Omega^{\prime}$ is the infinitesimal solid angle, into which radiation at position $x^{i}$ moving along direction $\hat{n}^{i}$ and with frequency $\nu$ is transported. $R^{\text {in/out }}$ depend on the underlying physical processes and in general do not allow for an analytic solution of the scattering integrals. The complexity of this double-integral is another major hurdle when attempting to solve the RTE numerically. In practice, many approximations are necessary for numerically treating the scattering term and an example for this will be shown in Sec. 5.2.2.

It is often useful to write the RTE in terms of the specific radiation intensity

$$
I_{(\nu)}:=\nu^{3} f_{(\nu)} .
$$

This can be done simply via multiplying Eq. (2.78) with $\nu^{3}$. Plugging in the collisional source, Eq. (2.79), and absorbing the factor $\nu^{3}$ in the emissivity, i.e., $\eta_{(\nu)}:=\nu^{3} \tilde{\eta}_{(\nu)}$, the RTE then becomes

$$
\left(\frac{\partial}{\partial t}+\hat{n}^{i} \frac{\partial}{\partial x^{i}}\right) I_{(\nu)}=\eta_{(\nu)}-\kappa_{a,(\nu)} I_{(\nu)}+\nu^{3} \mathcal{C}_{\text {scat }}
$$


The general-relativistic version of the RTE reads (Lindquist, 1966; Riffert, 1986; Mezzacappa \& Matzner, 1989; Shibata et al., 2011; Cardall et al., 2013)

$$
\left(p^{\mu} \frac{\partial}{\partial x^{\mu}}-\Gamma_{\nu \lambda}^{\mu} p^{\nu} p^{\lambda} \frac{\partial}{\partial p^{\mu}}\right) I_{(\nu)}=\mathcal{C}_{\mathrm{rad}}
$$

which is written in terms of the four-momentum $p^{\mu}=\mathrm{d} x^{\mu} / \mathrm{d} \tau$ with $\tau$ being the affine parameter of the radiation particle's trajectory.

\subsubsection{Solving the Radiative Transfer Equation}

As detailed above, the numerical solution of Eq. (2.82) poses a major challenge. Different strategies with different levels of approximations have been derived over the years (see e.g., O'Connor, 2015, for a short overview). The most common ones used in numerical astrophysics are summarized in form of a radar chart in Fig. 2.3. The decisive factors for distinguishing these methods are

Angular discretization: The probably biggest difference between different methods is the discrete treatment of the angular dependence. As discussed above, the RTE depends not only on time and space, but also on the direction of propagation of the radiation particles. This dependence can be completely ignored, approximated with only few directions of propagations, or be taken fully into account via multi-angular schemes. This factor also decides the level of accuracy for the treatment of radiation.

Computational cost: The previous point is inversely proportional to the computational cost of the method. Multi-angular schemes with higher accuracy typically require a higher computational effort. Nevertheless, this trade-off is not always straightforward and some schemes can achieve high accuracy at a relatively small computational cost.

Microphysical processes: Ideally, a radiative transport method includes all three types of reaction between fluid and radiation, i.e., emission, absorption, and scattering.

Physical applicability: Since the physical conditions in astrophysical systems reach from optically thick (diffusion limit) to optically thin (free-streaming limit), a desired feature of any radiative transport method is to accurately treat both of these extreme regimes and also the intermediate regime in between the two limiting cases.

Frequency dependence: The dependence of the specific intensity on the radiation frequency $\nu$ is often ignored - referred to as gray approximation - in order to save computational cost.

It should be noted that even within the same method, the level of sophistication with which the above points are being achieved can vary depending on the exact implementation. For instance, implementations vary in being Newtonion, post-Newtonion, special-relativistic or fully general-relativistic. For most high-energy astrophysical applications, the latter is strictly necessary, but also represents to most complex implementation. Another factor is the dimensionality. Given certain symmetries (like in core-collapse supernova simulations) one might chose a complex and computationally expensive method, but only implement it in one or two dimensions. These factors are 


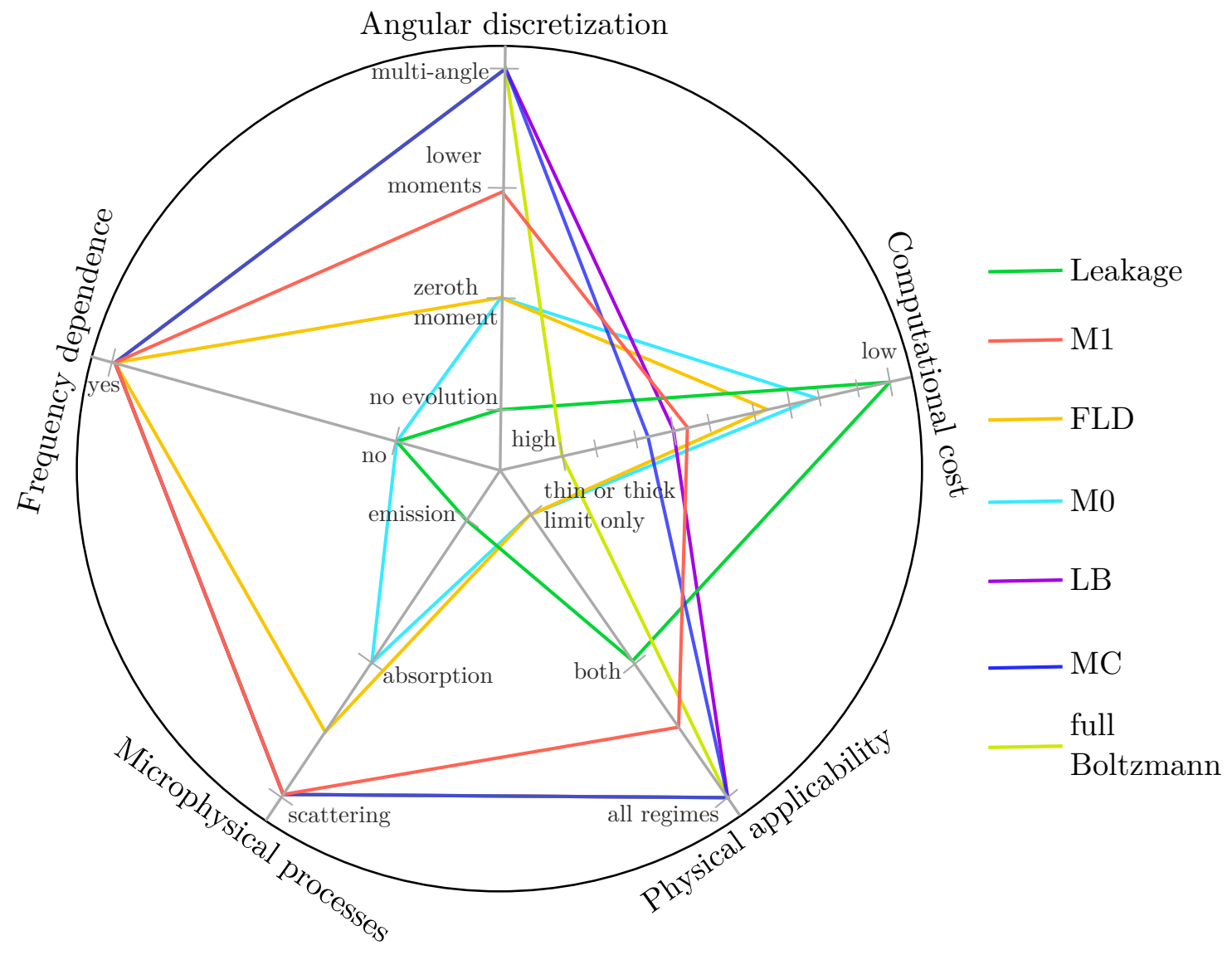

FIGURE 2.3: Summary of some of the most commonly used radiative-transfer methods in numerical astrophysics. See the main text for a detailed explanation of these methods.

common to all of the methods presented below and are thus not taken into account in Fig. 2.3.

We here briefly introduce each of the methods presented in Fig. 2.3 together with their advantages and disadvantages. As will become clear, there is no "best" method and the question about which method should be used depends on the specific problem at hand. Other methods that are not commonly used in numerical astrophysics like the spherical harmonics scheme (Radice et al., 2013), ray tracing (Deaton et al., 2018) or macroscopic streaming on Voronoi-Delauny grids (Paardekooper et al., 2010) will not be discussed here.

\section{Leakage}

The most approximate method and at the same time the one most commonly used in simulations of binary neutron star mergers is the leakage scheme (Ruffert et al., 1996; Rosswog \& Liebendörfer, 2003; Sekiguchi, 2010; Galeazzi et al., 2013; Perego et al., 2016). Within the leakage scheme, the distribution function of radiation particles is not evolved at all. Instead, the emission rates for energy and particle number are computed in the free-streaming (optically thin) and diffusion (optically thick) limits (Ruffert et al., 1996). The actual rates are then estimated via interpolating between these two limits. The resulting energy and lepton number is then subtracted from the fluid equations (2.61) via an additional source term (see also Sec. 2.3.3 for details on the coupling between fluid and radiation). Not having to evolve any additional variables makes this scheme easy to implement and computationally efficient. However, 
it only allows for the crude estimation of emission processes of monochromatic (gray) radiation, which are exact in the thin and thick limit, but not in the intermediate regime. It is typically used in simulations, where only cooling is relevant and heating via absorption of radiation energy can be neglected.

\section{M1}

The M1 method belongs to the larger family of moment-based methods (Thorne, 1981), within which the $N$ lowest moment of the distribution function are evolved using an analytic prescription for the $(N+1)$ th moment, which is required as closure relation since the evolution equation of the $N$ th moment depends on the next higher moment. M1, specifically, evolves the zeroth and first moment, i.e., the radiation energy and momentum density, and uses an analytic prescription for the pressure tensor. Evolving these moments allows to include emission, absorption and scattering effects in a selfconsistent way, making M1 much more accurate than the Leakage scheme. This comes at the prize of an increased computational cost, which is typically reduced via using the gray approximation (Rezzolla \& Miller, 1994; Zanotti et al., 2011; Fragile et al., 2012; Roedig et al., 2012; Sądowski et al., 2013; Fragile et al., 2014; Foucart et al., 2015; Sekiguchi et al., 2016; Skinner et al., 2019; Weih et al., 2020c). Nevertheless, a frequency-dependent implementation is also possible and indeed realized in O'Connor (2015); Anninos \& Fragile (2020).

Another aspect of different implementations of the M1 method is the closure relation used for obtaining the pressure tensor. A common approach is the Eddington closure (Zanotti et al., 2011; Fragile et al., 2012) allowing to only accurately treat the optically thick limit. The Levermore (Levermore, 1984) or Minerbo- ${ }^{6}$ (Minerbo, 1978) closures, on the other hand, are accurate in the thick as well as in the thin limit and provide a reasonable estimate for the intermediate regime, but come with a higher computational cost.

More details on the M1 method and how to implement it are given in Sec. 5.1 together with its application to realistic astrophysical problems.

\section{M0 and Flux-limited Diffusion}

The lowest-order approximation in the family of moment-based schemes would logically be called M0, since only the zeroth moment, i.e., the radiation energy density, is being evolved. However, this is typically referred to as flux-limited diffusion (FLD), while the only place where the term M0 is used in the literature is in Radice et al. (2016). This M0 scheme is special in the way that it splits the radiation into a trapped and free-streaming component following Liebendörfer et al. (2009). The trapped component is then treated using the leakage scheme, while for the free-streaming radiation the zeroth moment, i.e., the radiation energy density, is evolved in the optically thin limit (the closure required for the momentum density then simply follows from the assumption that radiation propagates radially along independent rays). This allows for an estimate of the radiation energy density and thus for the inclusion of absorption effects at a relatively small computational cost.

FLD, on the other hand, evolves the zeroth moment in the diffusion limit [Pomraning (1981); Levermore \& Pomraning (1981); see also Rahman et al. (2019) for

\footnotetext{
${ }^{6}$ Note that in the literature the Minerbo- 1 closure is often referred to as M1 closure. The term M1, however, denotes that the moments up to $N=1$ are being evolved. Consequently, a scheme evolving the zeroth and first moment, but not using the Minerbo-1 closure, is still an M1 scheme.
} 
an implementation of this scheme]. Due to the lack of the first moment, there is no information about the direction of propagation of the radiation. Consequently, FLD, as well as M0, are particularly well-suited for spherically symmetric problems. Within FLD radiation with different frequencies can also be coupled as is the case for M1 and is often referred to as multi-group flux-limited diffusion (MGFLD) (Bruenn et al., 1995). In contrast to M0, FLD also allows to include scattering - at least at a very approximate level given the lack of higher moments. This is due to the fact that scattering takes place in places of high optical depth, while there is no scattering for freely streaming radiation particles, as is the case in Radice's M0 scheme.

\section{Monte-Carlo Methods}

The previous methods either do not capture the angular dependence of the radiation distribution function or they do it in a limited way. Especially in simulations, where symmetries can not be applied, it is important to model the complete multi-angular dependence. One way to do so, is radiative transport via Monte-Carlo methods (MC) (Janka \& Hillebrandt, 1989; Ryan et al., 2015; Richers et al., 2017; Foucart, 2018; Miller et al., 2019b). MC is a statistical approach that samples the distribution with radiation packets. These packets are produced randomly with a probability that is derived from the underlying fluid's emissivity. Once the packet is produced, it is then propagated along geodesics until it is either absorbed or scattered, which is again modeled statistically deriving probabilities for both processes from the opacities computed from the fluid's properties. These processes are problematic for MC transport in regions of high optical depths; here the mean-free paths is much smaller than the grid resolution, so that a radiation packet would be scattered, emitted and absorbed many times during a single timestep. This can be circumvented by hybrid approaches (Foucart et al., 2018) or implicit MC (Fleck \& Cummings, 1971; Foucart et al., 2020).

For $N$ packets the distribution function $f_{(\nu)}\left(x^{i}, p^{i}, t\right)$ is then approximated at any time $t$ as

$$
f_{(\nu)} \approx \sum_{k=1}^{N} N_{k} \delta\left(x^{i}-x_{k}^{i}\right) \delta\left(p^{i}-p_{k}^{i}\right),
$$

where the $k$ th packet at location $x_{k}^{i}$ and with momentum $p_{k}^{i}$ represents $N_{\nu, k}$-many radiation particles with energy $\nu$. From the approximate distribution function, all physical quantities can then be derived.

The main advantage of this method is that it formally converges - albeit slowly - to a true solution of the RTE (Foucart, 2018). For reaching convergences and also in order to avoid statistical noise, a large number of radiation packets is necessary making MC transport unfeasible especially for three-dimensional problems. For this reason, MC transport is mostly used in simulations of core-collapse supernovae (Janka \& Hillebrandt, 1989; Janka, 1992), where symmetries can be exploited. For binary neutron star mergers MC transport has so far only been used on static snapshots neglecting a dynamical evolution and backreaction onto the fluid (Foucart et al., 2018) or in postmerger simulations with a stationary spacetime (Miller et al., 2019b). Only recently Foucart et al. (2020) have presented the first such simulation with a self-consistent evolution of neutrinos via $\mathrm{MC}$ transport for $\sim 5 \mathrm{~ms}$ after the merger. It is yet to be shown that MC transport is also feasible for the long-term evolution up to several $100 \mathrm{~ms}$.

\section{Lattice Boltzmann}


An alternative to $\mathrm{MC}$ transport that also covers the multi-angular dependence of the distribution function is the Lattice Boltzmann (LB) method for radiative transport. This method was recently developed (Weih et al., 2020a) as part of this Thesis and will be detailed in Sec. 5.2. It is based on the classical LB method, which is commonly employed in computational fluid dynamics (Krüger et al., 2017; Succi, 2018). The main idea is to discretize the momentum space in a way that allows to reconstruct the moments up to a desired order exactly, which is done via the computation of a suitable quadrature rule (see Sec. 5.2 or Gabbana et al. (2020) for more details). This discretization is then solved numerically via the stream-and-collide approach.

When applied to radiative transport, the LB method needs to be modified and loses some of its properties. It still allows for an accurate treatment of radiation in the diffusion limit and the intermediate regime at a relatively small computational cost. Even the free-streaming limit, the most challenging regime for LB, can be modeled accurately at a reasonable computational cost (Weih et al., 2020a). So far, the LB method has only been applied within the gray approximation and in special relativity. However, as for the moment-based methods the inclusion of frequency dependence is straight forward in exchange for a higher computational cost. An extension to curved spacetimes is less trivial and will be shortly addressed at the end of Sec. 5.3. Despite its limited application so far (see Sec. 5.2.4 for an astrophysical application of LB), LB has already proven to deliver more accurate results than M1 at a comparable computational cost.

\section{Full Boltzmann}

Finally, the RTE can also be solved directly. For doing so, the spatial and angular dependence of the distribution function is discretized, which is called $S_{N}$ method (Mezzacappa \& Bruenn, 1993; Yamada et al., 1999; Nagakura et al., 2018). Contrary to the LB method, the angular directions are naively discretized in the same way as space is. Furthermore, derivatives are solved using finite-differencing approaches in contrast to the stream-and-collide approach of the LB method. While direct solvers represent the most accurate method, they are limited by computational resources and thus are typically applied only in spherically (Mezzacappa \& Bruenn, 1993; Mezzacappa \& Messer, 1999) or axisymmetric (Nagakura et al., 2018) scenarios. Especially the supernova community is pushing towards fully three dimensional simulations. Indeed, recently Iwakami et al. (2020) presented the first (special-relativistic) supernova simulation in three-dimensional space using a full (low-resolution) seven-dimensional Boltzmann solver.

\subsubsection{Coupling between Radiation and Fluid}

As stressed throughout the previous sections, the inclusion of radiative transport in astrophysical simulations is important due to the backreaction of the radiation onto the fluid. This can significantly change the dynamics of the fluid and its composition (Rosswog, 2005; Perego et al., 2014; Fujibayashi et al., 2017; Miller et al., 2019a). In order to see how this affects the equations of GRMHD, we have to start again from Eq. (2.57), i.e., the conservation law for energy and momentum. In the presence of radiation the fluid energy-momentum tensor, which we so far denoted simply as $T^{\mu \nu}$, is no longer conserved. Instead, the total energy-momentum tensor will be conserved. We now use a more precise notation and write the total energy-momentum tensor

$$
T^{\mu \nu}=T_{\text {fluid }}^{\mu \nu}+T_{\text {rad }}^{\mu \nu},
$$


where we introduced the radiation energy-momentum tensor, $T_{\mathrm{rad}}^{\mu \nu 7}$, and the fluid energy-momentum tensor, which has so far simply been called $T^{\mu \nu}$ and is now more precisely denoted by $T_{\text {fluid }}^{\mu \nu}$. Note that the latter is composed into a matter and an electromagnetic contribution, i.e., $T_{\text {fluid }}^{\mu \nu}=T_{\text {matter }}^{\mu \nu}+T_{\mathrm{EM}}^{\mu \nu}$.

While Eq. (2.58) remains unchanged (baryons are neither produced nor destroyed through interactions with radiation), the energy and momentum of the whole system is now conserved. Consequently, Eq. (2.57) is rewritten as

$$
\nabla_{\mu} T^{\mu \nu}=\nabla_{\mu} T_{\text {fluid }}^{\mu \nu}+\nabla_{\mu} T_{\text {rad }}^{\mu \nu}=0 .
$$

In what is called radiation hydrodynamics the radiation and the matter are then treated as two distinct fluids described by the following equations of motion (Mihalas \& Mihalas, 1984)

$$
\begin{aligned}
\nabla_{\mu} T_{\text {fluid }}^{\mu \nu} & =-G^{\nu}, \\
\nabla_{\mu} T_{\text {rad }}^{\mu \nu} & =G^{\nu},
\end{aligned}
$$

where $G^{\nu}$ is the radiative four-force.

Radiation hydrodynamics is a macroscopic approach to treating radiation, where the radiation energy-momentum tensor is defined in terms of the macroscopic quantities $E, F_{i}$ and $P^{i j}$ being the radiation energy density, radiation momentum density and radiation pressure tensor, respectively. This is in contrast to the microscopical approach of the RTE described in Sec. 2.3.1. Since in numerical astrophysics the baryonic matter is typically treated macroscopically, i.e., via the GRMHD equations discussed in Sec. 2.2, Eq. (2.87) is solved via computing $G^{\nu}$ from the macroscopic radiation quantities. The radiation, however, can be either treated macroscopically or microscopically. The two approaches can be related to each other via the definition of the moments of the radiation distribution function, i.e.,

$$
M^{\mu_{1} \ldots \mu_{n}}=\int_{0}^{\infty} \mathrm{d} \nu \int_{4 \pi} I_{(\nu)} l^{\mu_{1}} \ldots l^{\mu_{n}} \mathrm{~d} \Omega,
$$

where $l^{\mu}$ is the radiation's direction of propagation in any reference frame (typically in the comoving fluid frame denoted by $l^{\mu}$, while $\hat{n}^{\mu}$ is the same quantity in the Eulerian frame). From a microscopic point of view $M^{\mu_{1} \ldots \mu_{n}}$ are simply the moments of the radiation distribution function, while from a macroscopic point of view, they can be identified with physical quantities: the zeroth, first and second moment correspond to $E, F_{i}$ and $P^{i j}$, while there is no more macroscopic interpretation for the higher moments. The radiation can now be evolved either via solving the RTE and then computing the moments of the distribution function, from which follows $G^{\nu}$ needed in Eq. (2.87) or via directly evolving the radiation moments according to Eq. (2.88). Both approaches will be discussed extensively in Chap. 5, where M1 represents a macroscopic and LB a microscopic method.

In any case, the explicit dependence of the radiation four-force on the moments has to be known for adding the radiative feedback as an additional source term to

\footnotetext{
${ }^{7}$ In the literature $T_{\text {rad }}^{\mu \nu}$ is often written as $R^{\mu \nu}$. We here stick to the former nomenclature in order to highlight the analogy to fluid dynamics.
} 
the conservative equations of GRMHD. Typically it is written as (Shapiro, 1996)

$$
G^{\alpha}=\int_{0}^{\infty} \mathrm{d} \nu \int_{4 \pi}\left[\eta_{(\nu)}+\eta_{s,(\nu)}-\left(\kappa_{a,(\nu)}+\kappa_{s,(\nu)}\right) I_{(\nu)}\right] l^{\alpha} \mathrm{d} \Omega .
$$

One can see that this expression is the "first moment" of the source term of the RTE. This is more evident when writing the scattering term $\mathcal{C}_{\text {scatt }}$ on the RHS of the RTE as

$$
\mathcal{C}_{\text {scat }}=\eta_{s,(\nu)}-\kappa_{s,(\nu)} I_{(\nu)}
$$

In this expression, scattering is approximated as an absorption-and-emission process, which is valid for isotropic and coherent scattering. With the definition of the total gray emissivity

$$
\eta:=\int_{0}^{\infty} \eta_{(\nu)}+\eta_{s,(\nu)} \mathrm{d} \nu
$$

and the energy-averaged total opacity

$$
\kappa:=\frac{\int_{0}^{\infty}\left(\kappa_{a,(\nu)}+\kappa_{s,(\nu)}\right) I_{(\nu)} \mathrm{d} \nu}{\int_{0}^{\infty} I_{(\nu)} \mathrm{d} \nu}
$$

the radiation four-force can be written in covariant form as (Farris et al., 2008)

$$
G^{\alpha}=\eta u^{\alpha}-\kappa_{a} E u^{\alpha}+\kappa F^{\alpha},
$$

where $\kappa_{a}$ is the gray absorption opacity defined equivalently to Eq. (2.93), but with the contribution of $\kappa_{a,(\nu)}$ alone.

The final equations of general-relativistic radiative-transport hydrodynamics (GRRTHD) following from Eq. (2.87) in conservative from and in terms of the $3+1$ decomposition of spacetime is the same as Eq. (2.61), but with the source vector now written as (Mihalas \& Mihalas, 1984; Shibata et al., 2011; Weih et al., 2020c)

$$
\boldsymbol{S}=\left(\begin{array}{c}
0 \\
\frac{1}{2} \alpha S^{i k} \partial_{j} \gamma_{i k}+S_{i} \partial_{j} \beta^{i}-E \partial_{j} \alpha-\alpha G^{\mu} \gamma_{\mu i} \\
\alpha S^{i j} K_{i j}-S^{j} \partial_{j} \alpha-\alpha G^{\nu} n_{\nu}
\end{array}\right)
$$

The emissivity and opacities depend on the exact interactions between radiation and fluid [see Rybicki \& Lightman (1986) for photons and Rampp (2000); Ruffert et al. (1996); Rosswog \& Liebendörfer (2003) for neutrinos]. Within this Thesis, the choice of interactions for different scenarios and the corresponding computation of $\eta$, $\kappa_{a}$ and $\kappa_{s}$ will be detailed throughout Chap. 5. 


\section{2 \\ Constraining the Equation of State of Nuclear Matter}

As already alluded to in Sec. 1.2, the first-ever detection of gravitational waves from a binary neutron star system, dubbed GW170817, was a milestone in that it heralded the beginning of the gravitational-wave multimessenger era. Besides this symbolic significance, the observation also yielded a plethora of information. In this chapter we review how this information can be used to infer properties of nuclear matter at densities outside the reach of earth-based experiments. In this regard, GW170817 has caused numerous publications on constraining the EOS of nuclear matter. After introducing the basic methods for deriving such constraints in Sec. 3.1, in Sec. 3.2 we disentangle this flood of information and compare all these constraints to the ones derived in Rezzolla et al. (2018a) and Most et al. (2018) (see Sec. 1.3 for the Author's contributions to these works). Furthermore, in Sec. 3.3 we show based on Weih et al. (2019) which neutron star mass ranges are optimal targets for future direct radius measurements via electromagnetic observations when combined with the gravitational-wave constraints from GW170817.

\subsection{Theoretical Neutron Star Models}

A unique characteristic of neutron stars is that they provide an environment of extreme gravity and equally extreme thermodynamic conditions. In order to describe these objects it is thus necessary to properly understand the theory of gravity and the nuclear interactions dictated by the strong force at extreme densities beyond nuclear saturation density. Vice-versa, this means that the observation of neutron stars can provide information on both of these fundamental forces of nature. For inferring this information from neutron star observables, it is necessary to model neutron stars theoretically and to compute observables from these theoretical models that can then be compared to observations. This is known as the relativistic inverse stellar structure problem (Lindblom, 2014). Long before the first detection of gravitational waves from a binary neutron star merger, a lot of research has been dedicated to solving this inverse stellar structure problem (Lindblom, 1992; Özel \& Psaltis, 2009; Read et al., 2009; Özel et al., 2010; Steiner et al., 2010; Lindblom \& Indik, 2012; Raithel et al., 2017). The main idea is that the relation between the pressure, $p$, and the density, $\rho$, of nuclear matter uniquely determines the two observables mass and radius of the neutron star (Özel \& Psaltis, 2009; Özel \& Freire, 2016; Lattimer \& Prakash, 2016). Hence, measuring these observables and working out the inverse stellar structure problem would then allow to infer the correct $p-\rho$ relation, i.e., the correct EOS 
(see Sec. 3.1.2), which in turn is determined by the fundamental interactions of the strong nuclear force.

\subsubsection{The Tolman-Oppenheimer-Volkoff equation}

The main ingredient for solving the inverse stellar structure problem is the computation of theoretical models of neutron stars, which will be detailed in this section.

We assume a neutron star to be a spherically symmetric (non-rotating), isotropic, ideal fluid body that is in hydrostatic equilibrium, i.e., the gravitational pull is balanced by the outwards directed hydrodynamic pressure. Such a model is known as Tolman-Oppenheimer-Volkoff (TOV) solution (Tolman, 1939; Oppenheimer \& Volkoff, 1939) and can be derived from the Einstein equations as follows.

Starting from a spherically symmetric ansatz for the metric with coordinates $r, \phi$ and $\theta$, i.e.,

$$
g_{\mu \nu}=\operatorname{diag}\left(-\mathrm{e}^{\nu}, \mathrm{e}^{\lambda}, r^{2}, r^{2} \sin ^{2} \theta\right)
$$

one can solve Eq. (2.10) for the metric potentials $\nu$ and $\lambda$, which are functions of the radial coordinate $r$ only.

Plugging this metric in the LHS of the Einstein equations, the energy-momentum tensor of a perfect fluid [cf., Eq. (2.60)] in the RHS and assuming a reference frame, in which the fluid is at rest, yields as only non-trivial equations (Shapiro \& Teukolsky, 1983; Weinberg, 1972; Rezzolla \& Zanotti, 2013)

$$
\begin{aligned}
\frac{\mathrm{d}\left(r \mathrm{e}^{-\lambda}\right)}{\mathrm{d} r} & =1-8 \pi e r^{2}, \\
\frac{\mathrm{d} \nu}{\mathrm{d} r} & =\mathrm{e}^{\lambda}\left(8 \pi p r+\frac{1}{r}\right)-\frac{1}{r},
\end{aligned}
$$

where we recall $e$ being the total energy density and $p$ the pressure of the perfect fluid. The first metric potential is obtained via integrating Eq. (3.2), i.e.,

$$
\mathrm{e}^{-\lambda}=1-\frac{8 \pi}{r} \int_{0}^{r} r^{2} e \mathrm{~d} r:=1-\frac{2 m(r)}{r},
$$

where $m(r)$ is for now simply a short-hand for

$$
m(r):=4 \pi \int_{0}^{r} r^{2} e \mathrm{~d} r .
$$

Furthermore, from the conservation of energy and momentum, i.e., Eq. (2.57), one can derive (Rezzolla \& Zanotti, 2013)

$$
\frac{\mathrm{d} p}{\mathrm{~d} r}=-\frac{e+p}{2} \frac{\mathrm{d} \nu}{\mathrm{d} r}
$$

Together with Eq. (3.3) and the expression for $\mathrm{e}^{-\lambda}$ the final equation of hydrostatic equilibrium reads

$$
\frac{\mathrm{d} p}{\mathrm{~d} r}=\frac{(e+p)\left[4 \pi p r^{3}+m(r)\right]}{r(2 m(r)-r)} .
$$

The set of equations (3.5) and (3.7) are referred to as TOV equations. For any EOS providing a relation between $p$ and $e$ a solution of this equation is uniquely 
determined by only one parameter. Usually this parameter is the central pressure, $p_{c}$, or the central rest-mass density, $\rho_{c}$. Starting from this central value, the TOV equations can be integrated from the star's center to its surface using any numerical method for solving ordinary first-order differential equations.

At the boundary of the star the metric given by Eq. (3.1) must be the same as that of a Schwarzschild black hole. This is a direct consequence of Birkhoff's theorem stating that the solution of a spherically symmetric metric in vacuum is unique (Birkhoff, 1923). Physically, the neutron star surface is defined by $p=0$. Comparing to the Schwarzschild metric, it is then evident that the star's gravitational mass can be computed as

$$
M=m(r=R)=4 \pi \int_{0}^{R} r^{2} e \mathrm{~d} r
$$

and that $m(r)$ represents the mass contained within a spherical shell of radius $r$. Thus, the two required observables, i.e., the neutron star mass $M$ and its radius $R$ are obtained via solving the TOV equations by integration outwards up to the point where $p=0$, which also defines $R$.

Another useful quantity is the baryon mass, $M_{\mathrm{b}}$. While this is not the mass that one would observe, it represents the sum of the rest-mass of all particles making up the neutron star. In other words, it does not include the contribution from the (negative) gravitational binding energy and is thus always larger than the gravitational mass $M$. Numerically it can be computed as (Bombaci, 1996)

$$
M_{\mathrm{b}}:=4 \pi \int_{0}^{R} \frac{r^{2} e}{\sqrt{1-\frac{2 m(r)}{r}}} \mathrm{~d} r .
$$

Subsequently, mass will always refer to gravitational mass, while the baryon mass will be stated as such explicitly. The difference between the two yields the gravitational binding energy

$$
\mathrm{BE}=M_{\mathrm{b}}-M
$$

\subsubsection{Soft and Stiff Neutron Stars: the Equation of State and Tidal Deformability}

The EOS has already been mentioned in Sec. 2.2 and in the previous subsection. In both cases it serves as the closure for a system of equations. In general, an EOS relates the thermodynamic quantities pressure $p$, rest-mass (energy) density $\rho(e)$, temperature $T$ and composition $Y_{e}$. For the high densities encountered inside neutron stars, i.e., up to $\sim 7 n_{\text {sat }}$, where $n_{\text {sat }}=0.16 \mathrm{fm}^{-3}$ is the nuclear saturation number density (Sakuragi, 2016), this EOS is determined by the strong nuclear force and many different approaches exist for modeling the EOS (see Oertel et al., 2017, for a recent review). In this section, the EOSs used for modeling cold neutron stars within this Thesis are described. It is also shown how it is connected to the tidal deformability, another quantity that can be inferred through gravitational waves. 


\section{Cold Neutron-Matter Equations of State}

Astronomically observable neutron stars are approximated to be cold, i.e., at zero temperature, because newly born neutron stars quickly cool due to the emission of neutrinos (Lattimer, 2015) reaching a temperature that is negligible compared to the typical temperatures encountered in high-energy astrophysics. Therefore, the equilibrium models described in the previous section are modeled with cold EOSs. Furthermore, neutron stars are in $\beta$-equilibrium (Alford \& Harris, 2018), so that the composition, i.e., the electron fraction, $Y_{e}$, which is equal to the proton fraction, is constant in time. The EOS for cold neutron star models is then a simple relation of the form $p=p(\rho)$.

At densities below nuclear saturation density the EOS can be constraint via chiral effective theory (CET) (Drischler et al., 2016, 2017). At ultra-high densities many times that found inside neutron stars - perturbative quantum chromodynamics (pQCD) can make exact predictions about the EOS (Kurkela et al., 2010; Fraga et al., 2014). The intermediate regime, however, is completely unknown and only restricted by a few basic physical considerations:

1.) The EOS has to be causal, i.e., the speed of sound, $c_{s}=\sqrt{\partial p / \partial e}$, is always below the speed of light.

2.) In order to ensure minimal thermodynamic stability, the pressure has to be a monotonically increasing function.

A third constraint comes from the observation of a pulsar with $M=2.01 M_{\odot}$ (Antoniadis et al., 2013). In order to be compatible with this observation, any valid EOS needs to be able to construct neutron star models (according to the TOV equations) that can yield at least this mass. A recent observation of an even heavier pulsar, i.e., PSR J0740+6620 (Cromartie et al., 2020), might provide a more stringent constraint. This measurement, however, is less precise and indeed the mass of $2.14 M_{\odot}$ has a large uncertainty.

Within the bounds of these three constraints, one can now construct an EOS as any mathematical function $p=p(\rho)$ in order to describe nuclear matter inside neutron stars. Three common approaches exist in the literature: piece-wise polytropes (e.g., Read et al., 2009; Hebeler et al., 2010; Raithel et al., 2016; Annala et al., 2018b; Most et al., 2018; Greif et al., 2019; Weih et al., 2019), constant speed-ofsound parametrizations (e.g., Alford et al., 2013; Tews et al., 2018, 2020; Capano et al., 2020; Dietrich et al., 2020a), and spectral representations (e.g., Lindblom, 2010; The LIGO Scientific Collaboration \& The Virgo Collaboration, 2017; Lindblom, 2018; Miller et al., 2020). For the work connected to this Thesis the piece-wise polytropic approach is used.

The EOSs are constructed in four parts as shown in Fig. 3.1 and detailed in the following:

1.) For the low-density part, i.e., the neutron star crust up to a number density of $n_{\text {crust }}=0.08 \mathrm{fm}^{-3}$, the EOS of Baym et al. (1971); Negele \& Vautherin (1973) is used.

2.) For densities $n>n_{\text {crust }}$ - as already detailed above - the EOS is well described by chiral effective field theory. We here use the EOS from Drischler et al. (2017), who derive the nuclear interactions for neutron matter using a nextto-next-to-next-to-leading order $\left(\mathrm{N}^{3} \mathrm{LO}\right)$ chiral expansion. This approach is unique in that it takes into account not only nucleon-nucleon interactions in a self-consistent way, but also the subleading three-nucleon (3N) interactions 
beyond the Hartree-Fock approximation. The $4 \mathrm{~N}$ interactions, on the other hand, are incorporated within the Hartree-Fock approximation (Kohno, 2013). This chiral expansion suffers from an increasing error at higher densities and the EOS only offers a reliable band (the relative error is $\lesssim 5 \%$ ) up until $n \approx$ $1.3 n_{\text {sat }}=0.21 \mathrm{fm}^{-3}$, which corresponds roughly to the densities found within the neutron star's outer core (oc). In practice, we fit the EOS of Drischler et al. (2017) with two piece-wise polytropes, i.e., we use

$$
\begin{aligned}
& p=K_{0}^{\mathrm{oc}} \rho^{\Gamma_{0}^{\mathrm{oc}}} \quad \text { for } \rho_{\text {crust }}<\rho<\rho_{0}, \\
& p=K_{1}^{\mathrm{oc}} \rho^{\Gamma_{1}^{\mathrm{oc}}} \quad \text { for } \quad \rho_{0}<\rho<\rho_{\text {match }},
\end{aligned}
$$

where $\rho_{\text {crust }}=n_{\text {crust }} m_{\mathrm{b}}$ and $\rho_{\text {match }}=1.3 n_{\text {sat }} m_{\mathrm{b}}$ with $m_{\mathrm{b}}$ being the mass of the nucleons for which we choose the atomic mass $m_{\mathrm{b}}=931.5 \mathrm{MeV}$. The polytropic constants of the outer core, $K_{i}^{\text {oc }}$, are chosen so that the first piece matches continuously to the crust, i.e.,

$$
K_{0}^{\mathrm{oc}}=\frac{\rho_{\text {crust }}^{\Gamma_{0}^{\mathrm{oc}}}}{p\left(\rho_{\text {crust }}\right)} .
$$

Following the same logic also for $K_{1}^{\text {oc }}$, the second piece then simply has to match the first one at $\rho_{0}=0.093 \mathrm{fm}^{-3}$, where $\rho_{0}$ is simply a best-fit value. The adiabatic indices, $\Gamma_{i}^{o c}$, are the free fit parameters. Using the upper and lower limits of the uncertainty band of the EOS gives as possible ranges $\Gamma_{0}^{\mathrm{oc}} \in$ $[1.31,1.58]$ and $\Gamma_{1}^{\mathrm{oc}} \in[2.08,2.38]$.

3.) For the high-density part we use

$$
p=\frac{3}{4 \pi^{2}}\left(\frac{\mu}{3}\right)^{4}\left(c_{1}-\frac{d_{1} X^{-\nu_{1}}}{\mu_{b} / \mathrm{GeV}-d_{2} X^{-\nu_{2}}}\right) .
$$

This is a fit-function for the cold quark-matter EOS derived by Fraga et al. (2014), which is based on the pQCD calculations of Kurkela et al. (2010). Note that this EOS is written in terms of the baryon chemical potential, $\mu$, instead of the density. The two are related via $\mu=(e+p) / n$. The fit parameters $c_{1}=0.9908, d_{1}=0.5034, d_{2}=1.452, \nu_{1}=0.3553$, and $\nu_{2}=0.9101$ are derived in Kurkela et al. (2014), where also the renormalization scale parameter $X$ is constrained to be within the interval $[1,4]$ and takes the uncertainty of the EOS into account, which increases when going from high to low density/chemical potential. Following Kurkela et al. (2014); Annala et al. (2018b), we define the high-density regime as the region where this error is still small $(\lesssim 5 \%)$, yielding $\mu>2.6 \mathrm{GeV}$.

4.) Finally, the low-density and high-density regimes are connected via an interpolation using piece-wise polytropes with four segments. The lowest segment is matched to the second outer-core segment at $\rho=\rho_{\text {match }}$ and the fourth segment is matched to Eq. (3.14) at $\mu=2.6 \mathrm{GeV}$. The remaining three matching points, $\rho_{i}^{\mathrm{ft}}$, between the segments of the four-trope (ft) can be chosen arbitrarily. Together with the four adiabatic indices of the four-trope, $\Gamma_{i}^{\mathrm{ft}}$, the polytropic constants, $K_{i}^{\mathrm{ft}}$, can be fixed.

In summary, the above described EOS has 10 free parameters:

2 parameters : $\quad \Gamma_{0}^{\mathrm{co}} \in[1.31 ; 1.58], \Gamma_{1}^{\mathrm{co}} \in[2.08 ; 2.38]$, 


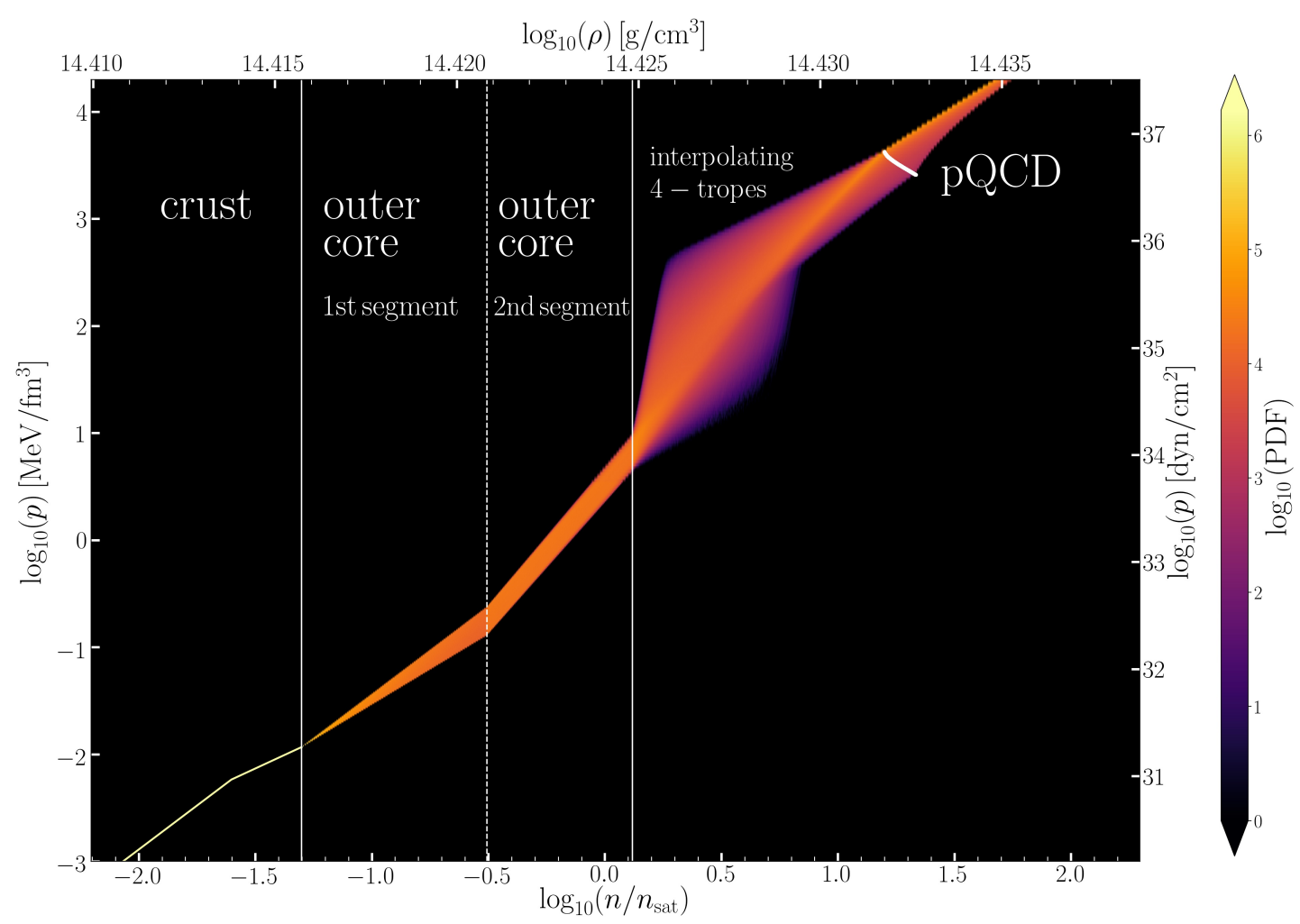

Figure 3.1: Probability distribution for uniformly sampled piece-wise polytropic EOSs in the pressure-density plane.

1 parameter : $\quad X \in[1 ; 4]$,

3 parameters : $\rho_{i}^{\mathrm{ft}} \in\left[\rho_{\text {match }} ; \rho(\mu=2.6 \mathrm{GeV})\right]$,

4 parameters : $\Gamma_{i}^{\mathrm{ft}} \in[0 ; \infty]$.

Any EOS constructed in this way automatically fulfills the criterion for minimal thermodynamic stability, i.e., $\partial p / \partial e>0$. The second condition, i.e., $c_{s}<1$, is enforced simply via discarding any combination of parameters that leads to a superluminal EOS. From each viable EOS one can construct a series of neutron star models using the TOV equations derived in Sec. 3.1.1. Computing the mass and radius of the models then yields a characteristic $M-R$ curve for each EOS. The third criterion, i.e., the EOS being required to yield a model with at least $M=2.01 M_{\odot}$, further rejects unphysical EOSs. The complete space of possible EOSs is shown in Fig. 3.1 in the $p$-n plane. The color encodes the probability distribution function (PDF) that is obtained when uniformly sampling the 10 free parameters as is done in Most et al. (2018); Weih et al. (2019) for a set of $\sim 10^{7}$ EOSs.

As a rule of thumb it can be said that EOSs at the lower edge of the distribution shown in Fig. 3.1, i.e., $p(\rho)$ having a small slope, produce more compact neutron stars with small radii. A large slope, on the other hand, leads to larger radii. This characteristic is known as softness/sitffness of the EOS and is one of the main features that distinguishes them ${ }^{1}$. Instead of thinking of the slope of the EOS, in the following it will be more convenient to think about small and compact stars (soft) versus large

\footnotetext{
${ }^{1}$ The terms stiff and soft relate to the fact that for a stiff EOS a small increase in density leads to a large increase in pressure. A fluid with this property would be difficult to compress, i.e., it would be stiff. A soft fluid, on the other hand, can easily be compressed since the pressure only increases marginally during the compression (Rezzolla \& Zanotti, 2013).
} 
stars (stiff). A quantity that is linked to this characteristic - and indeed can be used to measure the stiffness/softness - is the tidal deformability of a neutron star.

\section{The Tidal Deformability}

In this context the tidal deformability is a measure for how much a neutron star is deformed in the tidal field of a companion neutron star. To be more precise, it is the ratio of the induced quadrupole and the tidal field of its companion (Hinderer et al., 2010). The tidal field, $\mathcal{E}_{i j}$, and the quadrupole moment, $Q_{i j}$, arise from an asymptotic expansion of the metric at infinite distance from the neutron star. The $t t$-component of the asymptotic metric of a star with mass $M$ at a large distance $r$ is written as (Hinderer, 2008)

$$
g_{t t}=-1+2 \frac{M}{r}+3 \frac{Q_{i j}}{r^{3}} n^{i} n^{j}+\cdots-\mathcal{E}_{i j} r^{2} n^{i} n^{j}+\ldots,
$$

where $n^{i}=x^{i} / r, x^{i}$ being the spatial coordinates. From this expression $Q_{i j}$ can easily be identified as the quadrupole of the multipole expansion. To linear order and for static and spherically symmetric stars, this quadrupole is proportional to the tidal field, i.e.,

$$
Q_{i j}=-\lambda \mathcal{E}_{i j}
$$

where the constant $\lambda$ emerges as the tidal deformability. This constant can be computed numerically via introducing a static, even-parity, barotropic perturbation (Damour \& Nagar, 2009) to the spherically symmetric metric [cf., Eq. (3.1)] and splitting the resulting second-order differential equation of the perturbation into a set of two first-order ODEs of the perturbation $H(r)$ and the auxiliary $\beta(r)$ (Hinderer et al., 2010)

$$
\begin{aligned}
\frac{\mathrm{d} H}{\mathrm{~d} r}= & \beta, \\
\frac{\mathrm{d} \beta}{\mathrm{d} r}= & 2\left(1-2 \frac{m(r)}{r}\right)^{-1} H\left[-2 \pi\left(5 e+9 p+\frac{d e}{d p}(e+p)\right)\right. \\
& \left.+\frac{3}{r^{2}}+2\left(1-2 \frac{m(r)}{r}\right)^{-1}\left(\frac{m(r)}{r^{2}}+4 \pi r p\right)^{2}\right] \\
& +\frac{2 \beta}{r}\left(1-2 \frac{m(r)}{r}\right)^{-1}\left(-1+\frac{m(r)}{r}+2 \pi r^{2}(e-p)\right) .
\end{aligned}
$$

These two ODEs have to be solved together with the TOV equations (3.5) and (3.7) using the additional boundary conditions $H(r \rightarrow 0)=r^{2}$ and $\beta(r \rightarrow 0)=2 r$.

Once the quantity $y:=R \beta(R) / H(R)$ and the compactness $\mathcal{C}=m(R) / R$ are known, one can compute the dominant (corresponding to the $\ell=2$ mode) quadrupolar tidal Love number (Damour \& Nagar, 2009)

$$
\begin{aligned}
k_{2}= & \frac{8 \mathcal{C}^{5}}{5}(1-2 \mathcal{C})^{5}(2+2 \mathcal{C}(y-1)-y) \times\left\{3(1-2 \mathcal{C})^{2}[2-y+2 \mathcal{C}(y-1)] \ln (1-2 C)\right. \\
& \left.+4 \mathcal{C}^{3}\left[13-11 y+\mathcal{C}(3 y-2)+2 \mathcal{C}^{2}(1+y)\right]+2 \mathcal{C}[6-3 y+3 \mathcal{C}(5 y-8)]\right\}^{-1},
\end{aligned}
$$


which is related to the tidal deformability via

$$
\lambda=\frac{2}{3} k_{2} R^{5} .
$$

A small (large) value of the tidal deformability corresponds to EOSs that produce compact (large) neutron-star models. The advantage of this quantity is that it can be indirectly measured through gravitational waves. The phase, $\Psi$, of the gravitationalwave from a binary neutron-star merger is going to be affected by the finite size of the two stars once they get close enough (within the last few orbits before merger). This correction is formally of fifth post-Newtonian order and reads (Flanagan \& Hinderer, 2008)

$$
\delta \Psi=-\frac{117}{8} \frac{\left(\pi M_{\mathrm{tot}} f\right)^{5 / 3}}{\mu M_{\mathrm{tot}}^{4}} \tilde{\lambda},
$$

where $M_{\text {tot }}=m_{1}+m_{2}$ is the total binary mass, $\mu=m_{1} m_{2} / M_{\text {tot }}^{2}$ is the system's dimensionless reduced mass and $f_{\mathrm{GW}}$ the gravitational-wave frequency [cf., Eq. (2.52)]. Since the mass and frequency can be inferred already at early times, the phase correction only depends on the weighted tidal deformability

$$
\tilde{\lambda}=\frac{\left(12 m_{2}+m_{1}\right) m_{2} \lambda_{1}+\left(12 m_{1}+m_{2}\right) m_{1} \lambda_{2}}{26 m_{1} m_{2}},
$$

which, in turn, depends on the tidal deformabilities of the two component stars, $\lambda_{1}$ and $\lambda_{2}$ defined in Eq. (3.20). Note that in the literature typically the dimensionless quantities $\Lambda_{1 / 2}:=\lambda_{1 / 2} m_{1 / 2}^{-5}$ and

$$
\tilde{\Lambda}:=32 \tilde{\lambda} M_{\text {tot }}^{-5}=\frac{16}{13} M_{\text {tot }}^{-5}\left[\left(12 m_{2}+m_{1}\right) m_{1}^{4} \Lambda_{1}+\left(12 m_{1}+m_{2}\right) m_{2}^{4} \Lambda_{2}\right]
$$

are used (Favata, 2014), which will be also the quantities used in the remainder of this Thesis. The advantage of these definitions is that for equal mass ratio, $q:=$ $m 2 / m 1=1$, Eq. (3.23) reduces to $\tilde{\Lambda}=\Lambda_{1}=\Lambda_{2}$ and directly yields the desired tidal deformability, which can be related to the underlying EOS.

\subsubsection{Rotation and Stability}

Having discussed the TOV equations and the cold neutron-matter EOS, we have all ingredients for constructing neutron star models and comparing them to observations. However, there is one more property of neutron stars that will be important when analyzing the multimessenger signal of GW170817 in the next section. This property concerns the stability of neutron stars. All models computed via the TOV equations are stable in the sense that they are in hydrostatic equilibrium. This, however, is not a guarantee that these stars will be stable against small perturbations, just like a pen standing on its tip is a theoretically stable equilibrium, but in reality will fall due to the slightest perturbation. The same is true for some of the neutron star models. Specifically, the turning-point theorem by Friedman et al. (1988) states that on a line where the mass, $M$, is plotted as a function of the central rest-mass density, $\rho_{c}$, the point of maximum mass, i.e., the turning point, marks the onset of secular instability. This is visualized in Fig. 3.2, where the lower black line shows nonrotating models in the $M-\rho_{c}$ plane. All models on this line to the left of its maximum (marked with a black dot) are secularly stable models, while the ones on the right are secularly unstable and would, when perturbed, either collapse to a black hole or 


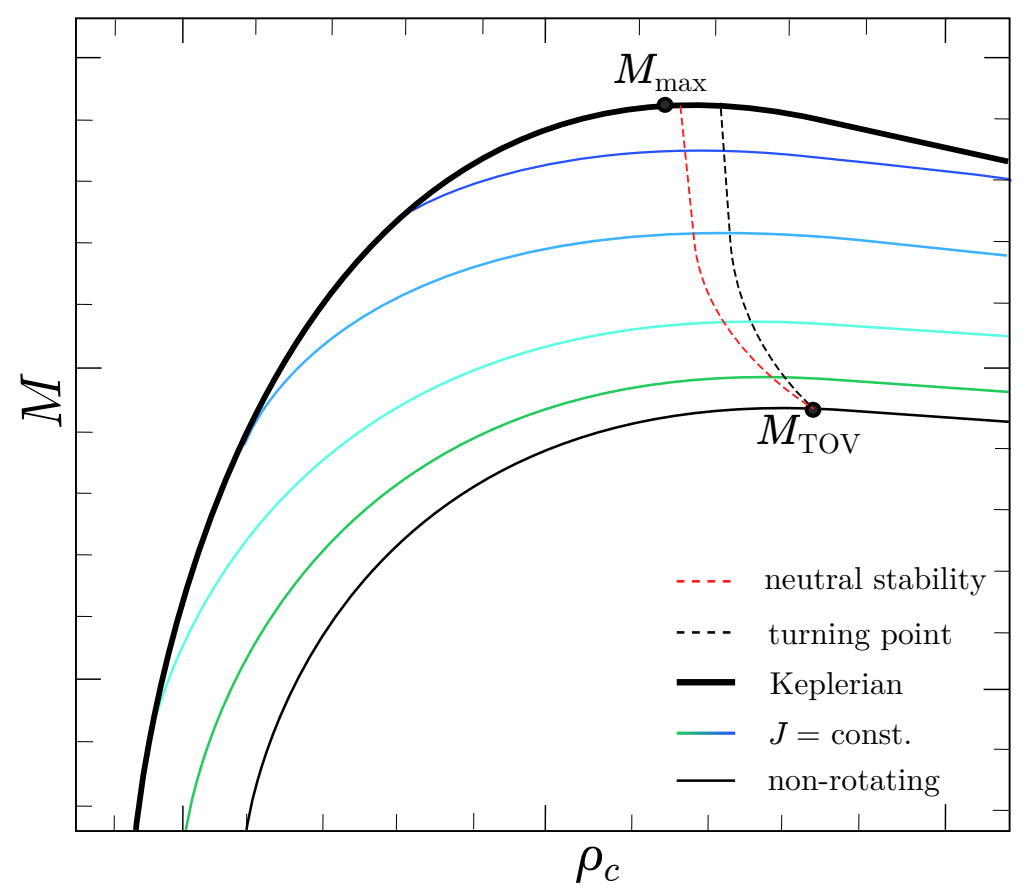

FiguRE 3.2: Mass over central rest-mass density plane for non-rotating and uniformly rotating neutron star equilibria.

migrate to the stable branch (depending on the direction of the perturbation). Secular instability refers to a timescale that is long compared to the typical dynamical time of stellar oscillations, but still much shorter than the lifetime of the star (Friedman \& Stergioulas, 2013). Since Friedman et al. (1988) also showed that dynamical instability sets in at higher densities than secular instability does, it is sufficient to consider only models on the left of the turning point as the ones to actually appear in nature.

This simple picture becomes a bit more complex after introducing rotation. The basic idea of computing equilibrium solutions of a rotating relativistic star is similar to that of non-rotating stars. Again, one uses an ansatz for the metric, this time an axisymmetric metric whose line element reads

$$
\mathrm{d} s^{2}=-\mathrm{e}^{2 \nu} \mathrm{d} t^{2}+\mathrm{e}^{2 \psi}(\mathrm{d} \phi-\omega \mathrm{d} t)^{2}+\mathrm{e}^{2 \eta}\left(\mathrm{d} r^{2}+r^{2} \mathrm{~d} \theta^{2}\right)
$$

and plugs it into the Einstein equations. This yields three elliptic equations, which can then be solved for the metric potentials $\nu, \psi, \omega$, and $\eta$ that depend only on the radial coordinate $r$ and the polar angle $\theta$, but not on the azimuthal angle $\phi$. Together with the equation for energy-momentum conservation, i.e., Eq. (2.57), where the energy-momentum tensor is again that of a perfect fluid, but this time written in the frame of a zero angular momentum observer (ZAMO), the equilibrium solution of a rotating neutron star can be obtained (see Friedman \& Stergioulas, 2013, for a detailed derivation of the equations). There are different strategies for solving these complex elliptic equations. The one used in this Thesis is the KEH scheme (Komatsu et al., 1989) implemented within the publicly available RNS code (Stergioulas \& Friedman, 1995).

In contrast to the spherically symmetric models computed from the TOV equations, rotating models have two free parameters, e.g., $\rho_{c}$ and the angular momentum, $J$. The colored lines in Fig. 3.2 show sequences of rotating neutron stars with $J$ being constant along each line and increasing from the bottom to the top line. Note that there is an upper limit on the angular momentum, i.e., an upper limit on how 
fast a neutron star can rotate. This so-called Keplerian limit is defined by the exact equilibrium between centrifugal forces on the star's surface and its gravitational pull. Were a model to rotate any faster, it would shed its mass due to the centrifugal force. This limit is marked by the top black line in Fig. 3.2.

For rotating neutron stars the turning-point criterion is only a sufficient, but not a necessary criterion and in fact the onset of instability happens at slightly smaller central densities. The exact location is marked by the neutral-stability line (see reddashed line in Fig. 3.2), which can be found via identifying the zero-frequency $F$-mode via simulating oscillating neutron stars (Takami et al., 2011). In practice, one typically uses the turning-point criterion (e.g., in Breu \& Rezzolla, 2016; Demircik et al., 2020; Dennison et al., 2019; Margaritis et al., 2020; Koliogiannis \& Moustakidis, 2020) since the difference to the neutral-stability line is only small and it is much easier to locate.

The maximum mass of rotating neutron stars, $M_{\max }$, is significantly higher than that of non-rotating stars due to the centrifugal forces that act against the gravitational pull. This maximum mass is always located on the Keplerian line, but is neither the end point of the turning-point, nor of the neutral-stability line. Instead, it is located at slightly smaller central rest-mass density (see Fig. 3.2). The slope of the $M-\rho_{c}$ curve, however, is very small around $M_{\max }$, so that for all practical purposes it is sufficient to consider the upper end of the turning-point line as the maximum.

Even higher masses can be supported when allowing for differential rotation. Differentially rotating neutron stars have a different angular velocity inside their center than they have at the surface. Depending on how the differential rotation is modeled, i.e., depending on the choice of the rotation law, many different models with different properties can be constructed (Ansorg et al., 2009; Gondek-Rosińska et al., 2017; Studzińska et al., 2016). It has been shown via numerical simulations that at least for the simple $j$-constant rotation law the neutral-stability line as well as the turning-point line can be extended for any given degree of differential rotation (Weih et al., 2018).

These stability properties of non-rotating, uniformly, and differentially rotating neutron star models are important when discussing the outcome of the binary neutron star merger GW170817, which will be subject of the next section.

\subsection{Constraints from the Gravitational Wave Event GW170817}

On August 17th, 2017, the LVC Network of gravitational-wave detectors measured for the first time the gravitational waves from the merger of a binary neutron star merger (Abbott et al., 2017). GW170817 represents a milestone in modern astrophysics and its significance has already been highlighted in Sec. 1.2. In this section we detail how the data obtained from this event was used to derive constraints on the EOS.

The relevant quantities measured by LVC are the chirp mass and the mass ratio. These quantities have been constrained to $\mathcal{M}=1.188_{-0.002}^{+0.004} M_{\odot}$ and $q=0.7-1.0$ for low-spin priors ${ }^{2}$ at a $90 \%$ confidence level (Abbott et al., 2017). From these quantities

\footnotetext{
${ }^{2}$ Low spin in this case means that the magnitude of the dimensionless spin, $\chi$, is smaller than 0.05 , which is a reasonable assumption given that most observed neutron star binaries have small spin (Lorimer, 2008; Zhu et al., 2018).
} 
the total binary mass

$$
M_{\text {tot }}=\mathcal{M}\left(\frac{q}{(1+q)^{2}}\right)^{-3 / 5}=2.74_{-0.01}^{+0.04} M_{\odot}
$$

could be inferred. What could not be determined, is the final fate of the merger product. This depends on a number of factors, most importantly the total binary mass (Bauswein et al., 2013; Koeppel et al., 2019), but also on more subtle details like the rotation profile of the remnant (Hanauske et al., 2017a), the energy transport due to neutrino radiation (Sekiguchi et al., 2016) and the role of magnetic fields in redistributing angular momentum throughout the remnant (Siegel et al., 2014; Palenzuela et al., 2015; Endrizzi et al., 2016).

This ignorance is illuminated by the electromagnetic counterpart of GW170817, highlighting the power of multimessenger astronomy. Only $\sim 1.7 \mathrm{~s}$ after the trigger for the gravitational-wave signal, a SGRB was detected of the same local origin (Goldstein et al., 2017; Savchenko et al., 2017; LIGO Scientific Collaboration et al., 2017). This not only proved the long-standing hypothesis of SGRBs being caused by binary neutron star mergers (Eichler et al., 1989; Narayan et al., 1992; Rezzolla et al., 2010; Berger, 2014), but also heavily implies that the merger remnant finally collapsed leaving behind a black hole-torus system. Indeed, most models require the collapse to a black hole as engine for powering an SGRB (Rezzolla et al., 2011; Murguia-Berthier et al., 2014), although alternative models like that of a magnetar engine (Metzger et al., 2008; Bucciantini et al., 2012; Metzger et al., 2018) can not be ruled out.

Furthermore, also the kilonova signal was detected (Coulter et al., 2017; Cowperthwaite et al., 2017; Villar et al., 2017) providing even more clues about the fate of the binary. This kilonova signal, dubbed AT2017gfo, consisted of an early blue and a late red signal, which can only be explained by two different types of mass ejecta (Barnes et al., 2016; Drout et al., 2017; Kasen et al., 2017). The early blue component is produced by the radioactive decay of lanthanide-poor material, whose formation requires an environment with high proton fraction $\left(Y_{p}=Y_{e} \gtrsim 0.25\right)$, while the red component originates from more neutron-rich material (Metzger, 2016; Bovard et al., 2017). The former is ejected from the remnant's hot surface at the poles (Metzger, 2017a,b). The large amount of blue ejecta required for modeling the observed kilonova requires a sufficiently long-lived ( $\gtrsim 100 \mathrm{~ms})$ remnant.

To summarize, the gravitational-wave detection together with its two electromagnetic counterparts provides three crucial pieces of information:

1.) From the gravitational-waves we know the total binary mass [see Eq. (3.25)].

2.) From the SGRB it can be inferred that the remnant collapsed to a black hole within at most $\sim 1.7 \mathrm{~s}$.

3.) However, the kilonova signal excludes a prompt collapse scenario, i.e., the remnant lived longer than $\sim 100 \mathrm{~ms}$.

\subsubsection{An Upper Limit on the Maximum Mass of Neutron Stars}

From these basic assumptions Rezzolla et al. (2018a) derived an upper limit on the maximum mass of a non-rotating neutron star, i.e., $M_{\text {TоV }}$, which can be translated into a constraint on the EOS. 


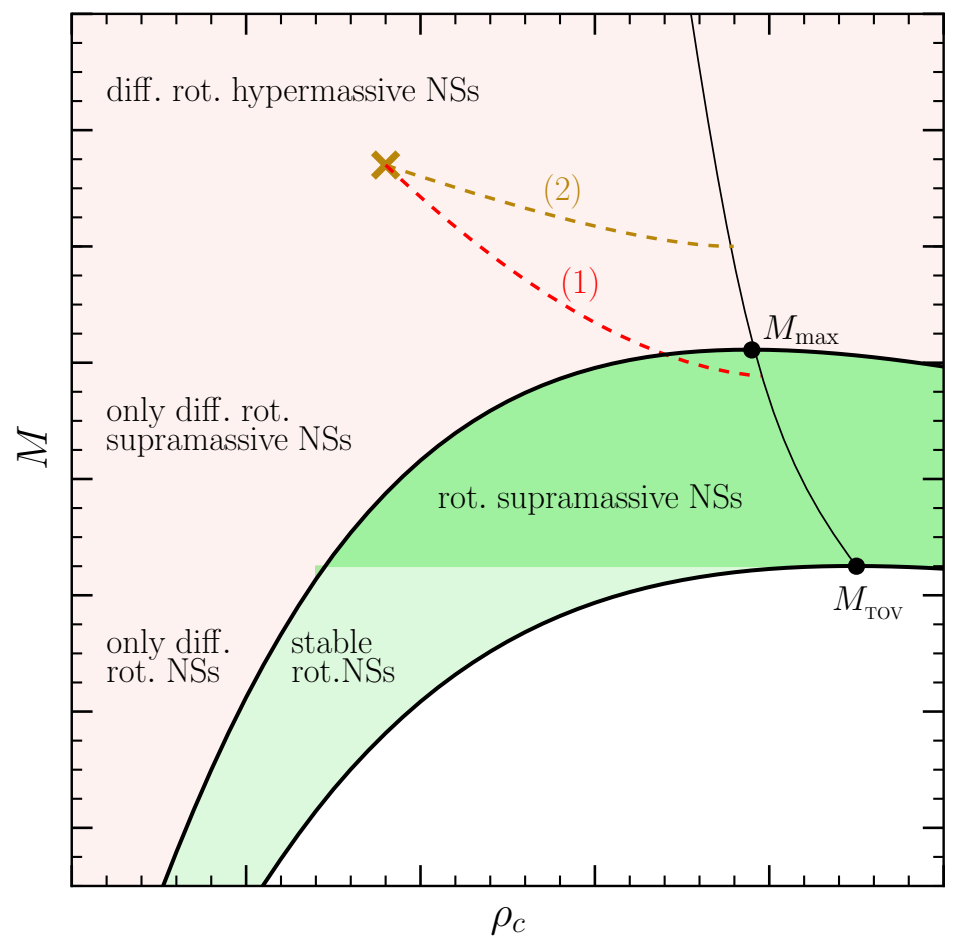

Figure 3.3: Possible trajectories (dashed lines) for the merger remnant of GW170817 in the $M-\rho_{c}$ plane. This plane is divided by the TOV and Keplerian lines (thick black lines) into regions for non-rotating stable (light green), uniformly rotating supramassive (green) and differentially rotating (red) stars. Models to the right of the stability-line (thin black line) are unstable and will collapse to a black hole. Reprinted from Rezzolla et al. (2018a) with permission by the American Astronomical Society.

The first step in deriving this upper bound requires an understanding of the trajectory of the merger remnant in the $M-\rho_{c}$ plane. This plane for equilibrium models of neutron stars was already introduced in Fig. 3.2 and is shown with additional details in Fig. 3.3. The product of the merger is a differentially rotating neutron star with mass $M_{\text {tot }}$ shown as a cross in Fig. 3.3. In order to collapse (cf., assumption 2) the remnant has to cross the stability line (thin black line) (Takami et al., 2011; Weih et al., 2018). This can happen in two different ways shown by two possible trajectories (dashed lines). Trajectory (1) shows the collapse as a uniformly rotating (supramassive) neutron star, while trajectory (2) indicates the collapse for a still differentially rotating (hypermassive) object. In both cases the remnant moves through the $M-\rho_{c}$ plane on lines of constant baryon mass. Considering the typical timescales for redistributing the angular momentum in order for the differential rotation to become uniform, trajectory (1) can be identified as the most-likely one. Indeed, from assumption 3 follows that the remnant could not have collapsed differentially rotating since the angular momentum is redistributed via effects like magnetic braking on a time scale of $\sim 100 \mathrm{~ms}$ (Shapiro, 2000; Hanauske et al., 2017a). In other words, if trajectory (2) were to be the correct one, the remnant would have collapsed much earlier, not leaving enough time to eject sufficient proton-rich material as inferred from the blue kilonova. On the other hand, the remnant can not have collapsed far below the maximum mass, $M_{\max }$, of uniformly rotating stars. This is due to the fact that, once uniformly rotating, the neutron star will only further spin down due to gravitational-wave (Usov, 1992) or magnetic-dipole (Zhang \& Mészáros, 2001) emission, both acting on time-scales much longer than the $\sim 1.7 \mathrm{~s}$ from assumption 
2.

We have now established that from assumptions 2 and 3 follows that the remnant likely moved through the $M-\rho_{c}$ plane following trajectory (1) in Fig. 3.3. From this it is possible to already derive an estimate on $M_{\max }$. For this we consider that the baryon mass, $M_{\text {tot,b }}$, along trajectory (1) is conserved, that a mass $M_{\mathrm{ej}}$ will be ejected, and that only the core of the remnant, i.e., the fraction $\xi$ of the total mass, will collapse. Since the collapse can not happen within a time of $\sim 1.7 \mathrm{~s}$ for models below $M_{\max }$, we can write as upper limit for the maximum baryon mass of uniformly rotating neutron stars

$$
M_{\max , b}<\xi M_{\mathrm{tot}, \mathrm{b}}-M_{\mathrm{ej}} .
$$

This can be converted into gravitational mass via multiplication with a factor $\eta:=M_{\mathrm{b}} / M$. This parameter only depends weakly on the EOS and can therefore be formulated in terms of a quasi-universal relation (Timmes et al., 1996; Breu \& Rezzolla, 2016). For $M=M_{\max }$ this dependence is especially weak, which can be seen from Fig. 3.4, where $\eta$ is plotted as a function of $M$ at the mass-shedding limit for six different realistic microphysical EOSs [DD2, SFHX, SFHO (Gaitanos et al., 2004; Hempel \& Schaffner-Bielich, 2010; Typel et al., 2010), SLY (Douchin \& Haensel, 2001), SK255 (Gulminelli \& Raduta, 2015), and APR4 (Akmal et al., 1998)]. The figure also shows that compared to the quasi-universal relation by Timmes et al. (1996) a much more precise relation can be obtained when concentrating on the case $M=M_{\max }$, i.e., $\eta=1.171$ with a standard deviation of $\sigma=6.8 \times 10^{-3}$. This value was also recently confirmed in an independent study by Koliogiannis \& Moustakidis (2020). Using this factor, the maximum gravitational mass of uniformly rotating neutron stars can be constraint as

$$
M_{\max }<\xi M_{\mathrm{tot}}-\eta^{-1} M_{\mathrm{ej}},
$$

where we used $M_{\text {tot }}=\eta^{-1} M_{\text {tot,b }}$.

Since it is much more difficult to connect an EOS to $M_{\max }$ than to $M_{\mathrm{TOV}}$, it is convenient to translate Eq. (3.27) into a bound on the latter. For this the quasi-universal relation by Breu \& Rezzolla (2016) can be used, connecting the two quantities via $M_{\text {max }}=\chi M_{\text {ТОV }}$, where $\chi=1.20_{-0.02}^{+0.02}$. This relation is well known and the precise value of $\chi$ has been computed by many independent studies within remarkable agreement (see e.g., Lasota et al., 1996; Lenka et al., 2017; Farajian et al., 2018; Bozzola et al., 2019; Shibata et al., 2019; Shao et al., 2020; Gao et al., 2020, for an incomplete list). Using this factor as final ingredient, the upper bound on the maximum mass of non-rotating neutron stars derived from GW170817 follows as

$$
M_{\mathrm{TOV}}<\chi^{-1}\left(\xi M_{\mathrm{tot}}-\eta^{-1} M_{\mathrm{ej}}\right) .
$$

The mas fraction in the core is estimated to be $\xi=0.95_{-0.06}^{+0.06}$ [see table II in Hanauske et al. (2017a)]. The parameter $M_{\text {ej }}$ follows from the consideration that the remnant core's mass ejecta can be associated with the blue ejecta (Metzger, 2017a; Shibata et al., 2017), which has been inferred to be $M_{\mathrm{ej}}=0.014_{-0.010}^{+0.010} M_{\odot}$ (Cowperthwaite et al., 2017; Drout et al., 2017). Furthermore, we account for the possibility of trajectory (1) to end not exactly at $M_{\max }$, but slightly below it, by using a conservatively small value of $\chi=1.15$ (see also Shibata et al., 2019, for an extended discussion on this parameter). Accumulating all parameters it follows that

$$
M_{\mathrm{TOV}}<2.16_{-0.15}^{+0.17} M_{\odot},
$$




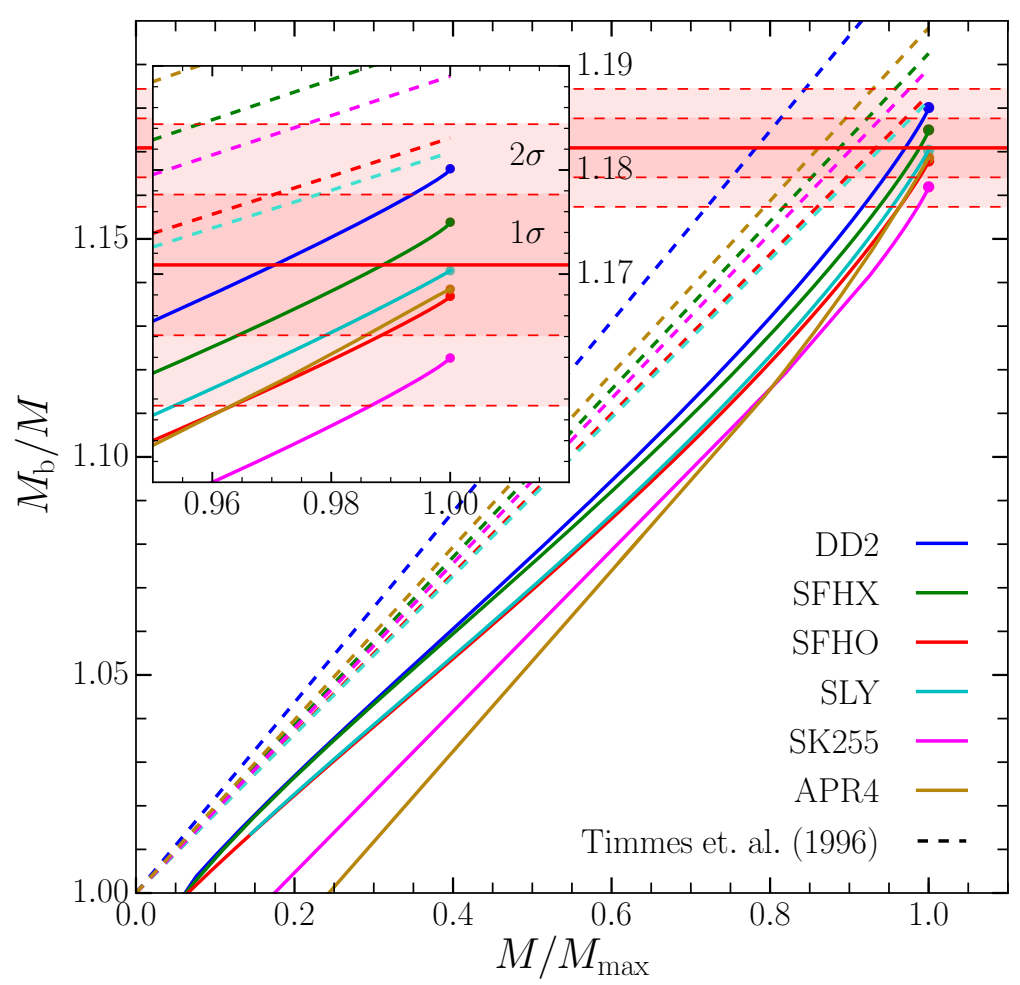

Figure 3.4: Conversion factor between baryon and gravitational mass, $\eta:=M_{\mathrm{b}} / M$ plotted as a function of gravitational mass for rotating neutron stars at the mass-shedding limit. Shown are six different realistic EOSs. The red horizontal line is the mean value over all EOSs at $M=M_{\max }$ (colored dots at the end of the curves) and the red bands denote the standard deviation. For comparison, also the quasi-universal relation by Timmes et al. (1996) is shown for the same EOSs with dashed lines. Reprinted from Rezzolla et al. (2018a) with permission by the American Astronomical Society.

where the errors correspond to twice the standard deviation computed with standard error propagation from the uncertainties in all of the above parameters.

This limit has been set by four different studies independently within a few weeks only (Margalit \& Metzger, 2017; Rezzolla et al., 2018a; Ruiz et al., 2018a; Shibata et al., 2017), all using different methods, but arriving at the same conclusion. Later on, Shibata et al. (2019) revisited the problem following a similar logic as in Rezzolla et al. (2018a), but with a detailed analysis of all the involved parameters and their error ranges. In this way, the value $M_{\text {то }} \lesssim 2.3 M_{\odot}$ was found as most conservative limit being in excellent agreement with the upper error range of Eq. (3.29).

\subsubsection{From the Tidal Deformability to the Neutron Star Radius}

Besides the chirp mass and the mass ratio, also the dimensionless weighted tidal deformability [see Sec. 3.1.2 and Eq. (3.23)] could be measured. Initially, LVC constrained its value to $\tilde{\Lambda}<800$ at a $90 \%$ confidence level (Abbott et al., 2017). From this Annala et al. (2018b) derived an estimate on the neutron star radius. Assuming a mass ratio of $q=1$ the constraint on $\tilde{\Lambda}$ is equivalent to a constraint on $\Lambda_{1.4}:=\Lambda_{1}=\Lambda_{2}$ 


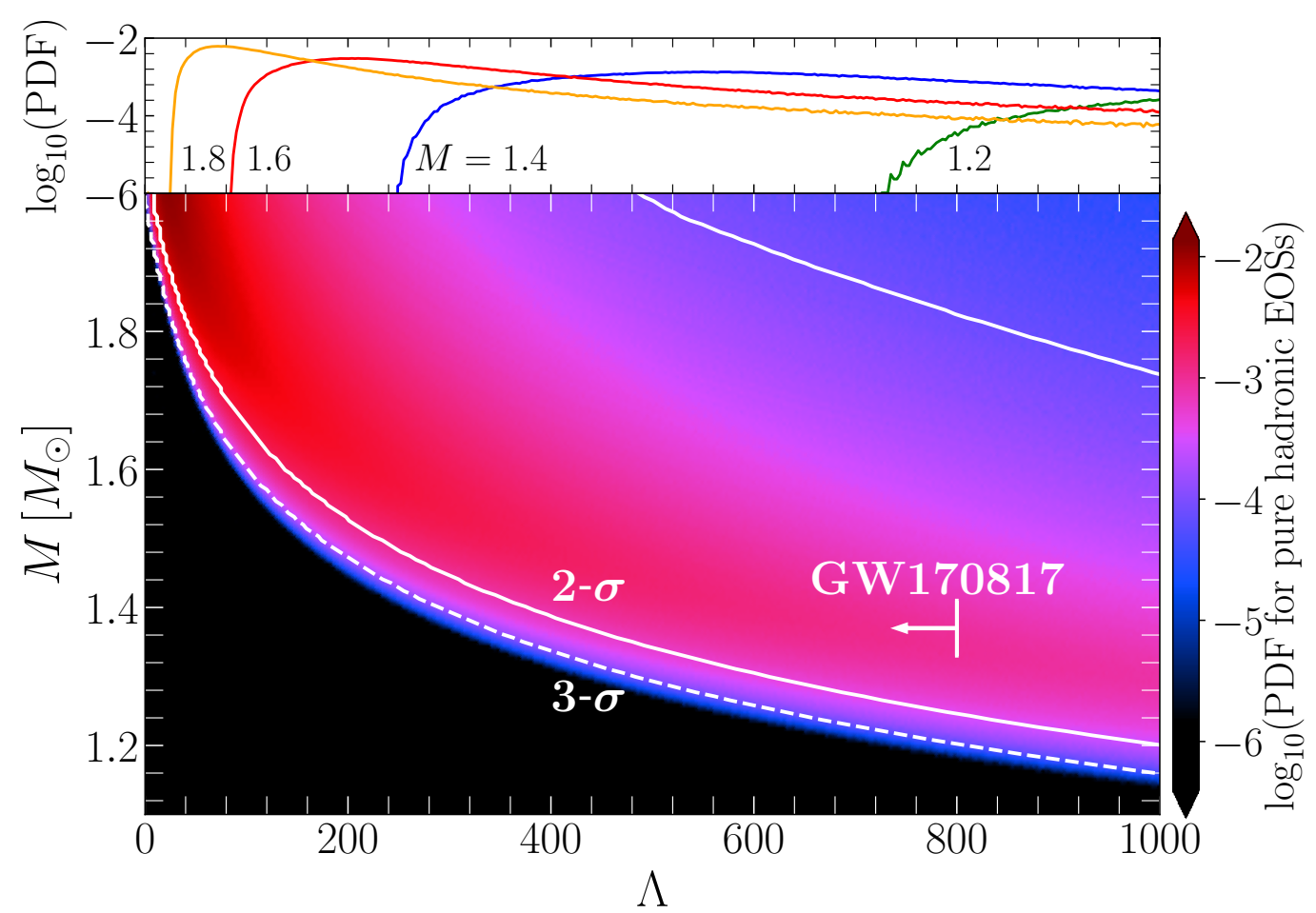

FIGURE 3.5: Probability distribution function (PDF) of a uniformly sampled dataset of EOSs constructed as outlined in Sec. 3.1.2 in the $M-\Lambda$ space. White solid (dashed) lines show the contours of the $2(3) \sigma$ confidence levels and the arrow denotes the limit of $\Lambda_{1.4}<800$ as comparison. On top, the accumulative distributions for different masses are shown. Reprinted from Most et al. (2018) with permission by the American Physical Society.

of the two component stars. Computing $\Lambda_{1.4}$ for a large set of EOSs using the extended TOV equations (see Secs. 3.1.1 and 3.1.2) they found that LVC's constraint on $\Lambda_{1.4}$ translates into the following constraint on the radius of a neutron star with a mass of $1.4 M_{\odot}: R_{1.4} \leq 13.6 \mathrm{~km}$. Shortly after this study, Most et al. (2018) used the so-far largest set of EOSs, which follows the parametrization outlined in Sec. 3.1.2, in a more detailed study. Besides the limit of $\Lambda_{1.4}<800$, we also use a lower limit of $\Lambda_{1.4}>400$, which was previously derived by Radice et al. (2018b). Using this and also applying the upper bound for the maximum mass, i.e., Eq. (3.29), as well as the lower bound of $M_{\mathrm{TOV}}>2.01 M_{\odot}($ Antoniadis et al., 2013), we find

$$
12.00 \mathrm{~km}<R_{1.4}<13.45 \mathrm{~km}
$$

at a $2 \sigma$ confidence level.

It should be noted that the lower limit $\Lambda_{1.4}>400$ is only based on a few EOSs and not a robust value. Disregarding this limit allows for somewhat smaller radii, i.e., $R_{1.4}>11.2 \mathrm{~km}$. A much more conservative lower bound on $\Lambda_{1.4}$ is found simply by plotting the entire distribution that is spanned by our set of viable EOSs. This is shown in Fig. 3.5, which color-codes the probability distribution for $\sim 2 \times 10^{7}$ uniformly sampled EOSs in the $M-\Lambda$ plane. It can be seen that there is a strict cutoff at small $\Lambda$ giving a lower bound of $\Lambda_{1.4}>375(290)$ at a $2(3) \sigma$ confidence level. This is consistent with the recent study by Kiuchi et al. (2019), in which $\Lambda_{1.4}<242$ was derived through numerical-relativity simulations.

Since the detection of GW170817 a large number of publications (see Tab. 3.1) has appeared in addition to the ones mentioned above setting constraints on the 


\begin{tabular}{|c|c|c|c|}
\hline Reference & $R_{1.4}[\mathrm{~km}]$ & $\Lambda_{1.4}$ & Note \\
\hline Annala et al. (2018b) & $<13.6$ & $>120$ & $\begin{array}{l}\text { Based on } 2.6 \times 10^{5} \text { piece-wise poly- } \\
\text { tropic EOSs. }\end{array}$ \\
\hline Most et al. (2018) & $\begin{array}{l}>11.2 \\
<13.45\end{array}$ & $>290$ & $\begin{array}{l}\text { Based on } 2 \times 10^{7} \text { piece-wise poly- } \\
\text { tropic EOSs; purely hadronic } \\
\text { EOSs. }\end{array}$ \\
\hline Most et al. (2018) & $\begin{array}{l}>8.53 \\
<13.74\end{array}$ & $>36$ & $\begin{array}{l}\text { Based on } 2 \times 10^{7} \text { piece-wise poly- } \\
\text { tropic EOSs; EOSs with phase } \\
\text { transition. }\end{array}$ \\
\hline Lim \& Holt (2018) & $\begin{array}{l}>10.42 \\
<12.80\end{array}$ & $\begin{array}{l}>136 \\
<519\end{array}$ & $\begin{array}{l}\text { Bayesian analysis with chiral effec- } \\
\text { tive field theory priors. }\end{array}$ \\
\hline Raithel et al. (2018) & $<13.0$ & - & Based on 6 realistic EOSs. \\
\hline Tews et al. (2018) & $\begin{array}{l}>9.0 \\
<13.6\end{array}$ & $\begin{array}{l}>80 \\
<580\end{array}$ & $\begin{array}{l}\text { Based on a speed of sound } \\
\text { parametrization and a minimal } \\
\text { model of the EOS. }\end{array}$ \\
\hline De et al. (2018) & $\begin{array}{l}>8.9 \\
<13.2\end{array}$ & $\begin{array}{l}>84 \\
<642\end{array}$ & $\begin{array}{l}\text { Based on a universal relation for } \Lambda \\
\text { derived from } \sim 10^{3} \text { piece-wise poly- } \\
\text { tropic EOSs. }\end{array}$ \\
\hline Malik et al. (2018) & $\begin{array}{l}>11.82 \\
<13.72\end{array}$ & - & $\begin{array}{l}\text { Based on a representative set of } 42 \\
\text { mean-field models. }\end{array}$ \\
\hline Abbott et al. (2018) & $\begin{array}{l}>10.5 \\
<13.3\end{array}$ & $<580$ & $\begin{array}{l}\text { Based on a spectral representation } \\
\text { of the EOS. }\end{array}$ \\
\hline Fattoyev et al. (2018) & $<13.76$ & - & $\begin{array}{l}\text { Based on a small set of realistic } \\
\text { EOSs. }\end{array}$ \\
\hline Radice et al. (2018b) & - & $>400$ & $\begin{array}{l}\text { Based on simulated collapse times } \\
\text { of only four EOSs. }\end{array}$ \\
\hline Montaña et al. (2019) & $\begin{array}{l}>10.1 \\
<13.11\end{array}$ & - & $\begin{array}{l}\text { Based on a parametrization that in- } \\
\text { cludes Category III twin stars. }\end{array}$ \\
\hline Burgio et al. (2018) & $\begin{array}{l}>10.7 \\
<13.1\end{array}$ & - & $\begin{array}{l}\text { Limit for } R_{1.5} \text { instead of } R_{1.4} \text {; in- } \\
\text { cludes two-family EOSs. }\end{array}$ \\
\hline Lim \& Holt (2019) & $\begin{array}{l}>10.26 \\
<12.87\end{array}$ & $\begin{array}{l}116, \\
557\end{array}$ & $\begin{array}{l}\text { Bayesian analysis with } 3 \times 10^{5} \text { en- } \\
\text { ergy density functionals as priors. }\end{array}$ \\
\hline Bauswein et al. (2013) & $>10.68$ & - & $\begin{array}{l}\text { Limit for } R_{1.6} \text { instead of } R_{1.4} \text {; } \\
\text { based on a quasi-universal relation } \\
\text { (from Newtonian simulations) for } \\
\text { the threshold mass to prompt col- } \\
\text { lapse. }\end{array}$ \\
\hline Koeppel et al. (2019) & $>10.92$ & - & $\begin{array}{l}\text { Based on a quasi-universal rela- } \\
\text { tion (from general-relativistic sim- } \\
\text { ulations) for the threshold mass to } \\
\text { prompt collapse. }\end{array}$ \\
\hline
\end{tabular}

TABLE 3.1: Constraints on neutron star radius and tidal deformability derived by various studies in the aftermath of the gravitational-wave event GW170817. Note that the credibility levels vary and are $90 \%, 2 \sigma(95.4 \%)$ or higher for all references. 
neutron star radius. Most notably, LVC themselves (Abbott et al., 2018) performed an analysis within which they refined their initial estimate to $\Lambda_{1.4}<580$ assuming both component neutron stars obey the same EOS.

A different method for inferring the neutron star radius has been used by Bauswein et al. (2017), who sets a lower limit on the threshold mass for prompt collapse (assuming the remnant in GW170817 did not collapse promptly). This threshold mass is related to the neutron star radius via a quasi-universal relation (Bauswein et al., 2013). Inverting this relations yields $R_{1.6} \gtrsim 10.68$. This estimate was later updated with a more accurate universal relation (based on fully general-relativistic simulations) by Koeppel et al. (2019) to $R_{1.6} \gtrsim 10.9 \mathrm{~km}$, which is again in agreement with Most et al. (2018).

We conclude this section by summarizing the flood of publications related to deriving constraints from GW170817 and comparing estimates on neutron star radii and tidal deformabilities in Tab. 3.1 (see also Raithel (2019) for a partial review).

\subsection{Optimal Mass Ranges for Future Constraints}

While the constraints discussed in the previous section come from a single gravitationalwave detection, there is also another way to measure astrophysical observables. The Neutron Star Interior Composition Explorer (NICER) (Arzoumanian et al., 2014; Gendreau et al., 2016) was launched in June 2017, just before the detection of GW170817, with the aim to measure neutron star radii via X-ray modeling (see Watts et al., 2016, for a review on X-ray timing). Indeed, most of the work done on the inverse stellar structure problem (Özel \& Psaltis, 2009; Read et al., 2009; Steiner et al., 2010; Raithel et al., 2017) was anticipating neutron star observables from such electromagnetic signals rather than the gravitational-wave signal discussed in the previous section. As it turned out, GW170817 came first and the lingering question of how much electromagnetic observations as the ones promised by the NICER mission could further constrain the EOS arose. In principle, a precise radius measurement NICER originally aimed at a precision with a 5\% relative error (Arzoumanian et al., 2014) - could yield equally strong constraints on the EOS as gravitational-wave detections (McNeil Forbes et al., 2019). Given GW170817 and everything that was learned from this event, the logical next step is combining the constraints of the previous section with electromagnetic observations. This is done in Fasano et al. (2019), where radius measurements from thermonuclear bursts in accreting neutron stars have been used to supplement the constraints from GW170817 (see also Özel et al., 2016; Guillot et al., 2013; Lattimer \& Steiner, 2014, for earlier studies that focussed on these radius measurements).

In this section we show how the already constrained EOS is further restricted by precise radius measurements as promised by the NICER mission. This is based on Weih et al. (2019), which was published before the publication of NICER's results (Guillot et al., 2019; Riley et al., 2019; Miller et al., 2019c). It can therefore be shown how the prediction compares to the constraints from the actual measurement. Furthermore, within this section optimal neutron star mass ranges are predicted for future radius observations.

In order to relate the observables to the EOS, we use the same dataset as described in Sec. 3.1.2 consisting of $\sim 2 \times 10^{7}$ EOSs. Note that it is important to use such a large dataset in order for the results to be convergent. A smaller dataset could still be used to infer information via Bayesian analysis which, however, sensitively depends 

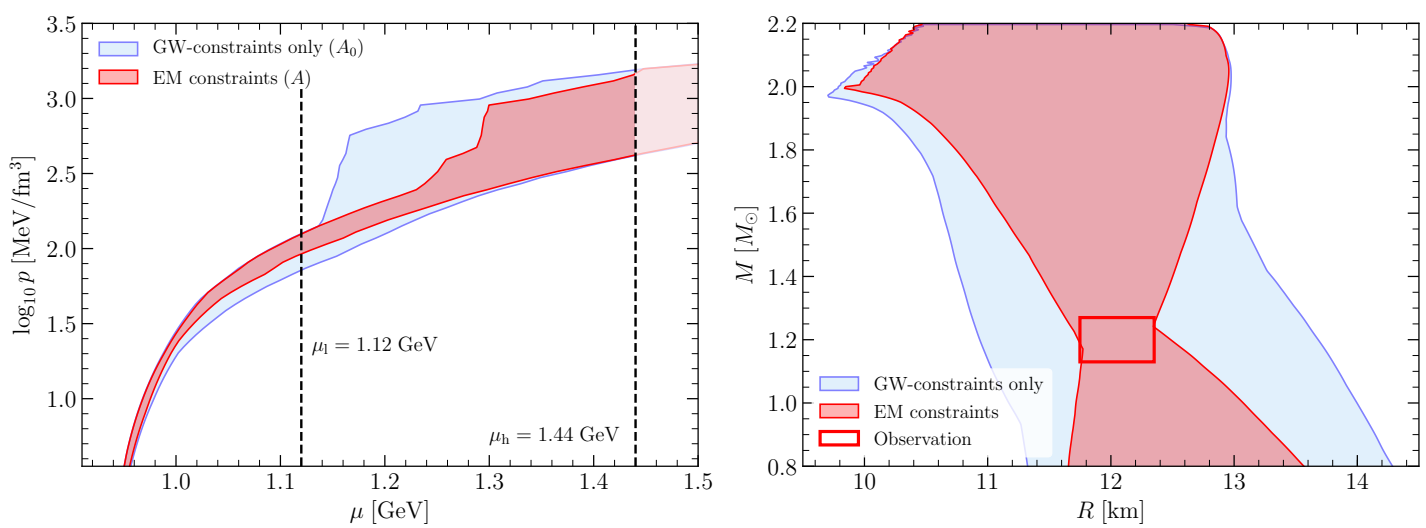

FiguRE 3.6: Left: Areas in the $p$ - $\mu$ plane spanned by all EOSs that fulfill the gravitationalwave constraints (1.) and (2.) (light-blue, $A_{0}$ ) and by EOSs that additionally fulfill a (hypothetical) constraint as expected from an electromagnetic measurement by NICER (red, $A$ ). The vertical dashed lines mark the chemical potentials that within this section define the lowand high-density regimes of the EOS. Right: The same areas, but in the $M-R$ plane, where the electromagnetic measurement is marked by a red box. An animated version of this figure is available online (Weih et al., 2019a). Reprinted from Weih et al. (2019) with permission by the American Astronomical Society.

on the prior assumptions (Greif et al., 2019). To our dataset we apply the constraints from GW170817 discussed in the previous section, i.e.,

(1.) The maximum mass reached by a sequence of non-rotating neutron stars. A conservative value that is generally agreed upon and within the bounds of Margalit \& Metzger (2017); Shibata et al. (2017); Rezzolla et al. (2018a); Ruiz et al. (2018a) is $M_{\text {тог }}<2.2 M_{\odot}$.

(2.) The tidal deformability of a $1.4 M_{\odot}$ star lies within the range $290<\Lambda_{1.4}<$ 580, where the upper limit is from LVC (The LIGO Scientific Collaboration et al., 2019) and the lower limit intrinsic to the dataset itself (see Fig. 3.5). This constraint is equivalent to the radius constraints discussed in the previous section.

Imposing these constraints reduces the set of EOSs considerably, leaving us with $\sim 20 \%$ of the complete set. This constraint-satisfying set of EOSs is publicly available (including the EOS and the neutron star observables) (Weih et al., 2019b) and shown as blue area in the pressure-chemical potential (left) and mass-radius (right) plane in Fig. 3.6.

In order to quantify how the EOS is further constrained by a potential radius measurement, we define a numerical quantity that measures the degree to which the EOS is constraint. To do so, we first define the reference area $A_{0}$, which is the area that is spanned by all EOSs that satisfy the constraints from GW170817 [see (1.) and (2.) ] in the $p-\mu$ space. Mathematically, this is written as

$$
A_{0}:=\int \mathrm{d} \mu \int \mathrm{d} p(\mu)=\int_{0}^{\mu_{h}}[\max (p(\mu)-\min (p(\mu)))] \mathrm{d} \mu,
$$

where $\mu_{h}=1.44 \mathrm{GeV}$ is the highest value for the chemical potential for which the EOS can be probed. We choose this cutoff because this corresponds to the highest density ${ }^{3}$

\footnotetext{
${ }^{3}$ The highest number density within our sample is $\sim 6.5 n_{\text {sat }}$.
} 
found within any of the neutron star models in our dataset and densities beyond that can not be probed with neutron stars. By changing the limits of the above integral the reference area can be varied. We will here probe the low- and high-density regime of the EOS with the reference areas $A_{0, l}$ and $A_{0, h}$ defined by the integration interval $\left[0 ; \mu_{l}=1.12 \mathrm{GeV}\right]$ and $\left[\mu_{l} ; \mu_{h}\right]$ (see dashed lines in Fig. 3.6), respectively.

Any radius measurement poses an additional constraint on the EOS and will thus decrease the total space spanned by all viable EOSs in the $p$ - $\mu$ plane. In other words, imposing a measurement by a box in the $M-R$ plane, whose size depends on the error of the measurement, will discard all EOSs that do not yield TOV models that fall within this box. The new area, $A$, associated with this more-constrained set can then be compared to the reference area, $A_{0}$. In this way the degree of constraint (DOC) is defined, i.e.,

$$
\chi:=1-\frac{A}{A_{0}} .
$$

The concept of comparing these two areas is visualized in Fig. 3.6, which is also available as animated version (Weih et al., 2019a). From the definition of the DOC follows that $\chi \ll 1$ corresponds to a measurement that is not constraining the EOS at all (in comparison to the constraints we already know), while $\chi \simeq 1$ would indicate a measurement that significantly constrains the EOS, i.e., causing the red area in Fig. 3.6 to shrink to a line. Since the DOC compares the total areas, which themselves are convergent for our EOS library, it presents a quantity that is independent of any prior assumptions for the construction of the EOSs.

Considering the primary targets originally aimed at by the NICER mission (Arzoumanian et al., 2014; Watts et al., 2016) the following three scenarios are considered:

Case 1: The radius of only one of the primary targets, i.e., PSR J0437-4715 (Johnston et al., 1993), with a mass of $1.44 M_{\odot}$ will be successfully measured.

Case 2: The radius of both primary targets, i.e., PSR J0437-4715 and PSR J0030+0451 (Lommen et al., 2000) will be successfully measured. The mass of the latter is unknown and we here assume a massive star with $2.0 M_{\odot}$.

Case 3: Same as Case 2, but assuming 1.2 $M_{\odot}$ for PSR J0030+0451.

Using the method outlined above we can now analyze to which degree the different cases would constrain the EOS. For this we place a box in the $M-R$ plane at the hypothetically measured radius with an extent that corresponds to a $5 \%$ relative error (Arzoumanian et al., 2014) and a fixed error of $\pm 0.07 M_{\odot}$ for the mass ${ }^{4}$, which is motivated by the typical accuracy of known neutron-star masses (Özel \& Freire, 2016).

The resulting DOC is visualized in Fig. 3.7 for all three cases (top to bottom). Focusing on the top panel (Case 1) we can see in the middle that a measurement of $R=12.28 \pm 0.31 \mathrm{~km}$, which is the most likely value reported by Most et al. (2018) for this mass, would only marginally constrain the EOS, i.e., yields a DOC of $\chi \simeq 0$. This is surprising since this measurement would clearly reduce the possible space in the $M-R$ plane (see inset), but not at all in the $p-\mu$ space, where the red and the blue areas are the same. The situation improves when the measured radius is $10 \%$ smaller (left panel), yielding $\chi \approx 0.67$, while a $10 \%$ larger radius (right panel) again only yields a marginal constraint. In other words, measuring the radius of a $1.44 M_{\odot}$ star would only provide a significant constraint if the radius turns out to be $R_{1.44} \lesssim 12 \mathrm{~km}$.

\footnotetext{
${ }^{4}$ Qualitatively similar results are also obtained with a larger error of $\pm 0.14 M_{\odot}$.
} 


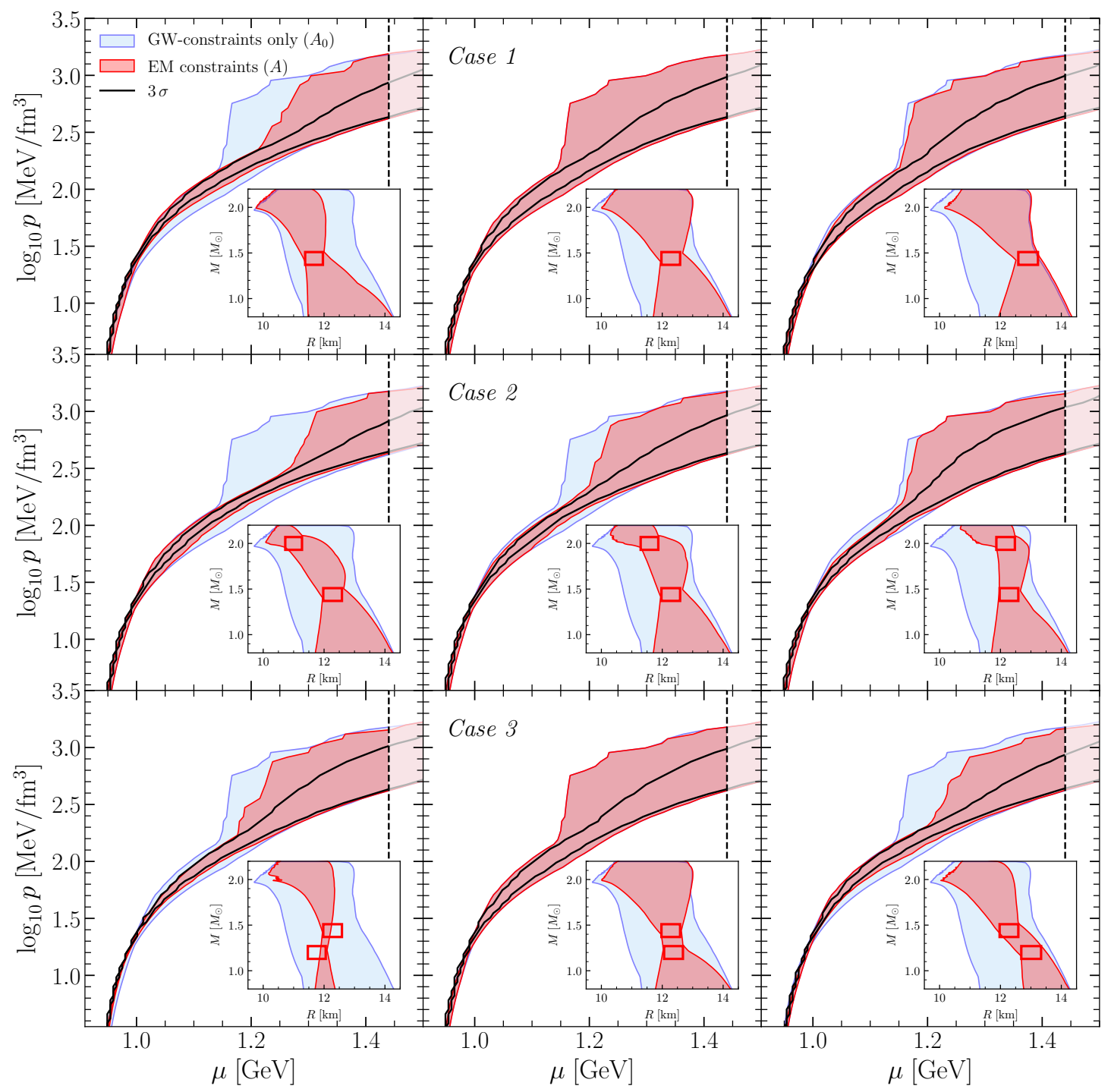

Figure 3.7: Same as Fig. 3.6 but for the three reference cases Case 1-Case 3 reported top to bottom. While the central column always assumes the measured radius to be the most likely one according to Most et al. (2018), the left (right) columns report the DOC when the measured radius is assumed to be $10 \%$ smaller (larger). The black solid lines show the $3 \sigma$ confidence levels of the EOSs' probability distribution obtained from a Bayesian analysis. Reprinted from Weih et al. (2019) with permission by the American Astronomical Society.

Similar conclusions are true also for Cases 2 and 3, which are reported respectively in the middle and bottom row of Fig. 3.7. In these cases the radius for PSR J0437-4715 is fixed at its most likely value, while for PSR J0030+0451 a small (left), most likely (middle), and large (right) measured radius is explored. As reference, we also show the $3 \sigma$ contours (black solid lines) obtained by computing the possibility distribution function via a Bayesian analysis where we use the maximum of a bivariat Gaussian distribution within the red boxes in the $M-R$ plane for the likelihoods (as done in e.g., Raithel et al. (2017)) and as prior the distribution obtained in Most et al. (2018). These confidence levels show that even in the case of $\chi \simeq 0$, constraints can still be obtained in statistical terms within a certain confidence level. As already mentioned, however, this will depend on the choice of prior entering the Bayesian analysis (Greif et al., 2019). We therefore use the DOC as defined in Eq. (3.32) that - assuming a convergent EOS library as is the case here - is free of the bias introduced by the prior 


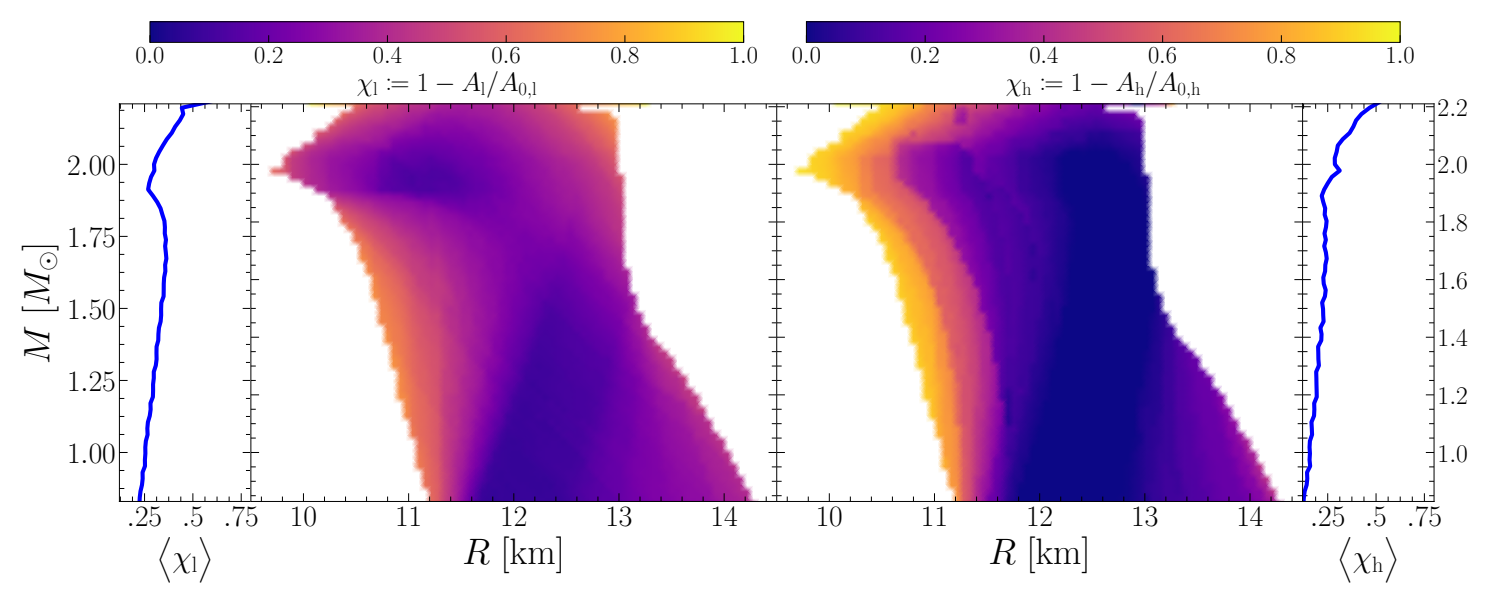

FiguRE 3.8: Degree of constraint (DOC) color-coded in the $M-R$ plane for all possible mass and radius measurements. The color at any point in the plane encodes the DOC for a measurement centered around this point. Left and right panels distinguish the DOC for the low $\left(\chi_{l}\right)$ and high-density $\left(\chi_{h}\right)$ regime of the EOS (see dashed lines in Fig. 3.6 for the definitions of these regimes). The blue lines denote the radial averages of $\chi_{l / h}$ according to Eq. (3.33). Reprinted from Weih et al. (2019) with permission by the American Astronomical Society.

since it compares the outer limits of the covered EOS space.

Fig. 3.7 only shows the DOC for specific radius measurements, but in principle a mass-radius observation could result in a box anywhere in the $M-R$ plane. Fig. 3.8 shows the DOC color-coded for all possible measurements. The figure should be understood in the way that the color at any point encodes the DOC for a measurement whose error box is centered at this point (see also the animation in Weih et al., 2019a). Note that the two panels in Fig. 3.8 distinguish the DOC for the low- (left panel) and high-density (right panel) part of the EOS. It is clear that both regimes of the EOS would best be constrained if a small radius (see the left, yellow-orange area) would be measured. Of course one can not choose beforehand which radius actually will be measured. However, it might be possible to choose which mass range to observe since often the mass of a neutron star can be determined independently, e.g., via Shapiro delay (Lattimer \& Prakash, 2004; Demorest et al., 2010). The average of the DOC for every given mass, i.e.,

$$
\left\langle\chi_{l / h}\right\rangle(M):=\frac{1}{R_{\max }-R_{\min }} \int_{R_{\min }}^{R_{\max }} \chi_{l / h}(M, R) \mathrm{d} R
$$

is shown as blue line on the left (right) in Fig. 3.8. These averages show that the best constraints can be expected from observing the most massive neutron stars. This was to be expected since the most massive neutron stars reach the highest densities for which the EOS is the least known. Interestingly, the averaged DOCs also reveal that for the low-density regime there is a local maximum around $M \sim 1.8 M_{\odot}$, meaning that - besides the most massive neutron stars - targets with $M \sim 1.7-1.8 M_{\odot}$ promise the best constraints compared to other mass ranges. This local maximum is likely connected to the already known constraint of $M_{\text {Tог }}>2.01 M_{\odot}$ (Antoniadis et al., 2013) that is incorporated into our EOS dataset. This result thus perfectly reflects the motivation of this study, i.e., which observation provides the best constraints given the constraints we already know.

Following the same logic we can now analyze the impact of additional radius 

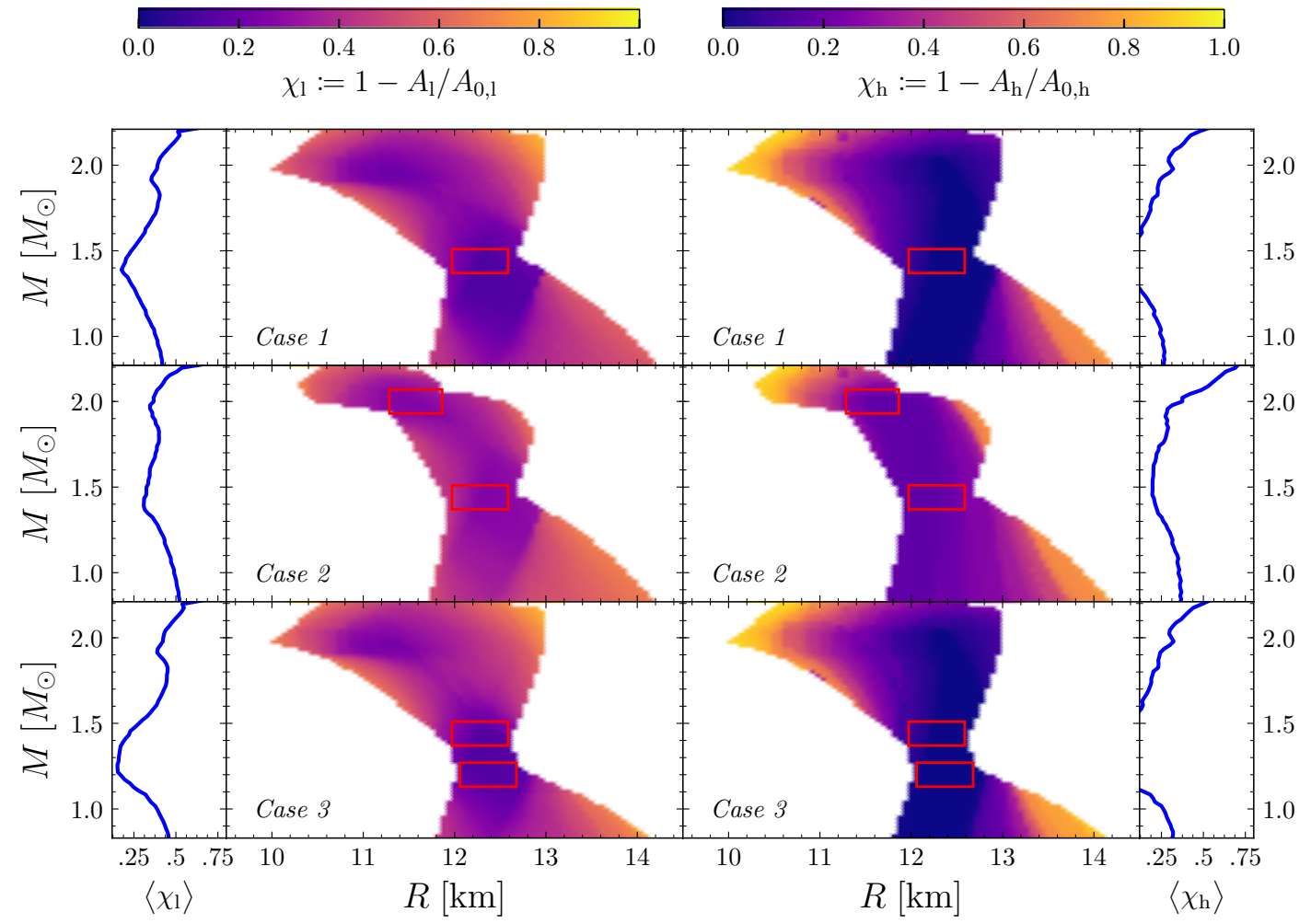

Figure 3.9: Same as Fig. 3.8, but for additional radius measurements assuming already known constraints (red boxes) from observations according to Cases 1-3 (top to bottom). Reprinted from Weih et al. (2019) with permission by the American Astronomical Society.

measurements. In particular, Fig. 3.9 shows the DOC in the same way as Fig. 3.8 does, but for Cases 1-3. The red boxes mark an observation as expected from the NICER mission and the color then encodes the additional constraints that can be obtained from a further observation beyond the NICER mission. The local maximum for $\left\langle\chi_{l}\right\rangle$ around $\sim 1.8 M_{\odot}$ is again present in all three cases.

Furthermore, the averaged DOC $\left\langle\chi_{l / h}\right\rangle$ suggests that a radius measurement of a low-mass star with $M \lesssim 1.1 M_{\odot}$ might yield constraints as promising as the ones that can be obtained from a high-mass star. These large values for the DOC can be attributed to the potential measurement of a large radius for low-mass stars (see the orange tail in the lower right, i.e., for $R>13.5 \mathrm{~km}$ that is significantly more distinct for $\chi_{h}$ than for $\chi_{l}$ ). The high values of the DOC in this area contribute to a large average. This large average, however, does not take into account that in our EOS sample such large radii are extremely rare. In other words, it is highly unlikely that such a large radius would actually be measured and thus that such a significant constraint on the EOS would be obtained.

One should then rather ask the following question: at which mass range can the best constraints be expected on average and how likely is it that a radius yielding good constraints will actually be measured? In order to answer this question, a more precise quantity to look at would be a probability weighted average of the DOC. This does again introduce the problem of the prior, which will bias the result. Notwithstanding this, Fig. 3.10 shows an example of what such a probability-weighted DOC, i.e., $\chi_{l / h} \cdot \mathcal{P}$, and its average, $\left\langle\chi_{l / h} \cdot \mathcal{P}\right\rangle$, looks like using Case 1 as an example. $\mathcal{P}$ is the probability distribution function in $M-R$ space, for which we use the information from Most et al. (2018). Comparing the weighted averages (dashed blue lines) with the 


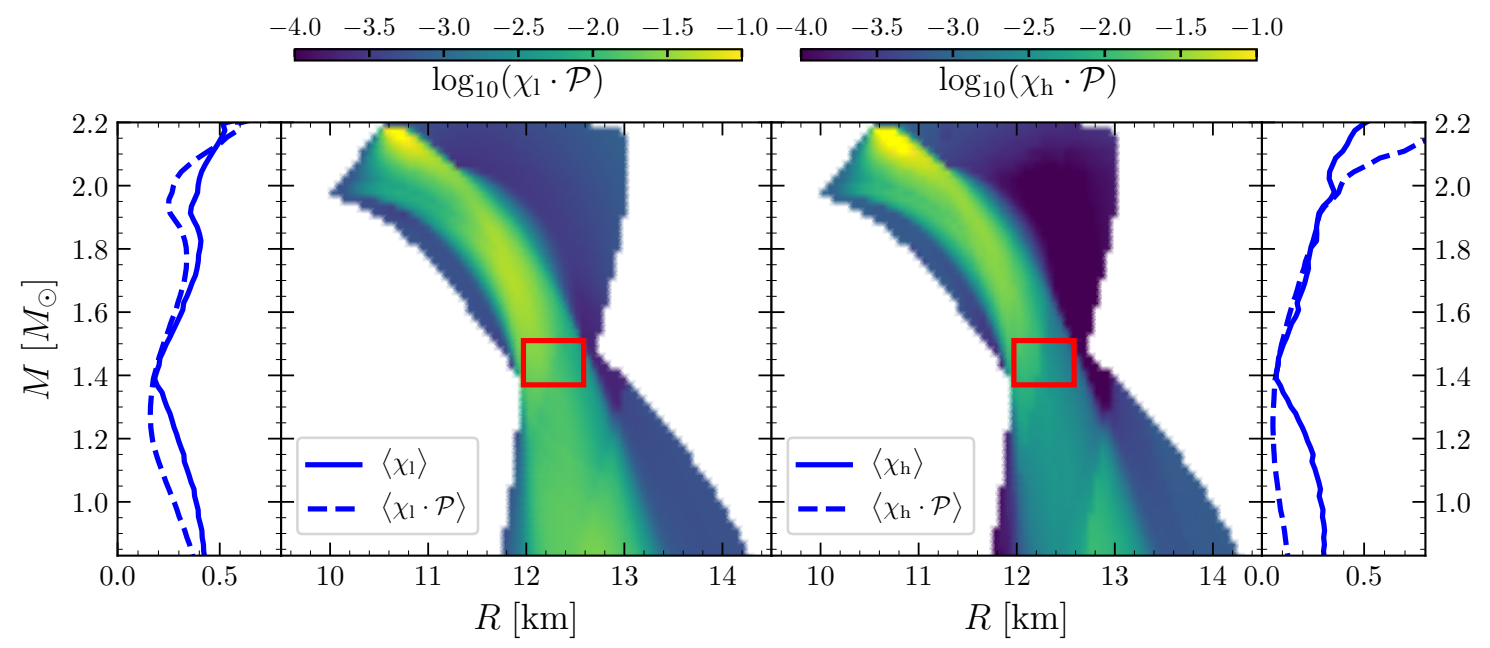

Figure 3.10: Same as the top panel of Fig. 3.9, but for a DOC convoluted with a probability distribution $\mathcal{P}$. The left and right panels this time show the probability weighted averages of the DOC (dashed lines) and compared it to averages shown in Fig. 3.9 (solid lines). Reprinted from Weih et al. (2019) with permission by the American Astronomical Society.

previously obtained averages from Fig. 3.9 (repeated as blue solid lines in Fig.3.10 for a convenient comparison) it is clear that the high average of $\chi_{h}$ for low-mass stars is much smaller when probability weighted. The local maximum for $\chi_{l}$ around $\sim 1.8 M_{\odot}$, on the other hand, is still present and thus can be considered as a robust result. This becomes also clear when looking at the DOC in the $M-R$ plane in Fig. 3.9, which is much more uniformly distributed over the entire radius range for $\chi_{l}$ around $M \sim 1.8 M_{\odot}$ as it is the case for $\chi_{h}$ for low masses, where the DOC drastically varies for different radii. Of course, the average of such a smooth distribution does not change with any probability-weighting (independent of the prior), while the weighting can have a huge impact on a non-uniformly distributed DOC. The same results have also been found for Case 2 and Case 3.

In summary, it can be said that the best constraints for the low-density regime of the already constrained EOS can be obtained when targeting neutron stars with $M \sim$ $1.8 M_{\odot}$. The high-density regime, on the other hand, will be best constrained when targeting the most massive stars, while low-density stars with $M \lesssim M_{\odot}$ might also provide significant constraints, but only when unlikely large radii will be measured.

\subsubsection{Comparison to Results from the NICER Mission}

In December 2019 the NICER collaboration published the results of their direct pulsar observations in a series of seven papers (Riley et al., 2019; Raaijmakers et al., 2019; Bilous et al., 2019; Bogdanov et al., 2019a,b; Miller et al., 2019c; Guillot et al., 2019). Indeed, the radius of PSR J0437-4715 and a simultaneous mass-radius measurement for PSR J0030+0451 could be obtained and have been reported as $R=15.3_{-1.6}^{+2.0} \mathrm{~km}$ for the former and $M=1.34 M_{\odot}, R=12.7 \mathrm{~km}$ for the latter (Bogdanov et al., 2019a,b). These values, however, are obtained from standard phase-resolved spectroscopy, which is a very limited analysis, and especially the values for PSR J0030+0451 only present fixed parameters for a Markov Chain Monte Carlo method. A more detailed analysis using full phase-channel inference has been conducted with independent codes for different models for the X-ray emitting hot spots in Riley et al. (2019) and Miller et al. (2019c). Unfortunately, these studies are restricted to PSR J0030+0451, for 
which an independent mass measurement is not available. The mass and radius have been found to be $M=1.34_{-0.16}^{+0.15} M_{\odot}, R=12.71_{-1.19}^{+1.14} \mathrm{~km}$ (Riley et al., 2019) and $M=1.44_{-0.14}^{+0.15} M_{\odot}, R=13.02_{-1.06}^{+1.24} \mathrm{~km}$ (Miller et al., 2019c), each at a $1 \sigma$ confidence level.

Combining these numbers, the error obtained over both models is much larger than the one assumed in the above analysis (Weih et al., 2019). Nevertheless, these measurements can still be used to constrain the EOS as done in Raaijmakers et al. (2019) resulting in a rather large constraining region in the $M-R$ plane. Applying the above outlined method and computing the corresponding DOC yields a value of $\chi_{l}=0=\chi_{h}$. In other words, not a single EOS in our sample could be ruled out from the NICER measurement. This is due to the fact that the EOS is already significantly constrained by GW170817 and compared to these constraints the NICER results can not provide any new information (in absolute terms; they still can in statistical terms after performing a Bayesian analysis).

While this is unfortunate, the NICER results still yield an independent observation that complements GW170817 and is completely consistent with the gravitationalwave constraints. Furthermore, the NICER mission provided also other type of information, e.g., on the shape of a pulsar's magnetic field (Bilous et al., 2019). More importantly, seven new targets could be identified (Guillot et al., 2019) providing promising candidates for future observations.

\subsection{Summary and Outlook}

In this chapter was shown that the EOS of nuclear matter could be significantly constrained in the recent years following the first detection of gravitational waves from a binary neutron star merger. Two major conclusions could be drawn from this event that provide these constraints. First, an upper limit on the maximum mass of non-rotating neutron stars was set. Following the general consensus about the merger remnant collapsing to a black hole, the value $M_{\text {то }}<2.3 M_{\odot}$ is now generally agreed upon as most conservative upper limit. Secondly, the tidal deformability of a non-rotating neutron star with mass $\sim 1.4 M_{\odot}$ is constraint to $\Lambda_{1.4}<580$, which is equivalent to a radius constraint of $R_{1.4} \lesssim 13.74 \mathrm{~km}$.

The agreement on a lower limit for tidal deformability and radius, on the other hand, is much weaker. This is mostly due to the fact that the EOS can exhibit a phase transition from purely hadronic to quark matter. This would significantly soften the EOS leading to much smaller radii (see e.g., Most et al., 2018; De et al., 2018; Montaña et al., 2019; Alvarez-Castillo et al., 2019; Christian et al., 2018; Annala et al., 2020). One major problem is that the constraints following GW170817 are mostly based on the inspiral gravitational-wave signal. A potential phase transition, however, might take place at higher densities (Christian \& Schaffner-Bielich, 2020) that are only reached during the postmerger evolution. This possibility will be discussed exhaustively in the next chapter.

Combining all currently known constraints (including GW170817, pulsar observations, chiral effective field theory, and $\mathrm{pQCD}$, but not including the vague limit of $\Lambda_{1.4}>400$ ) significantly reduces our uncertainty on the EOS of nuclear matter. This is shown in Fig. 3.11, where the left panel shows the same as Fig. 3.1, i.e., our uncertainty in the $p-\rho$ space before GW170817. The right panel shows how this uncertainty has been reduced through GW170817.

Since then at least one more binary neutron star merger could be observed, i.e., GW190425 (Abbott et al., 2020a) with a total mass of $\sim 3.4 M_{\odot}$. Such a high mass 


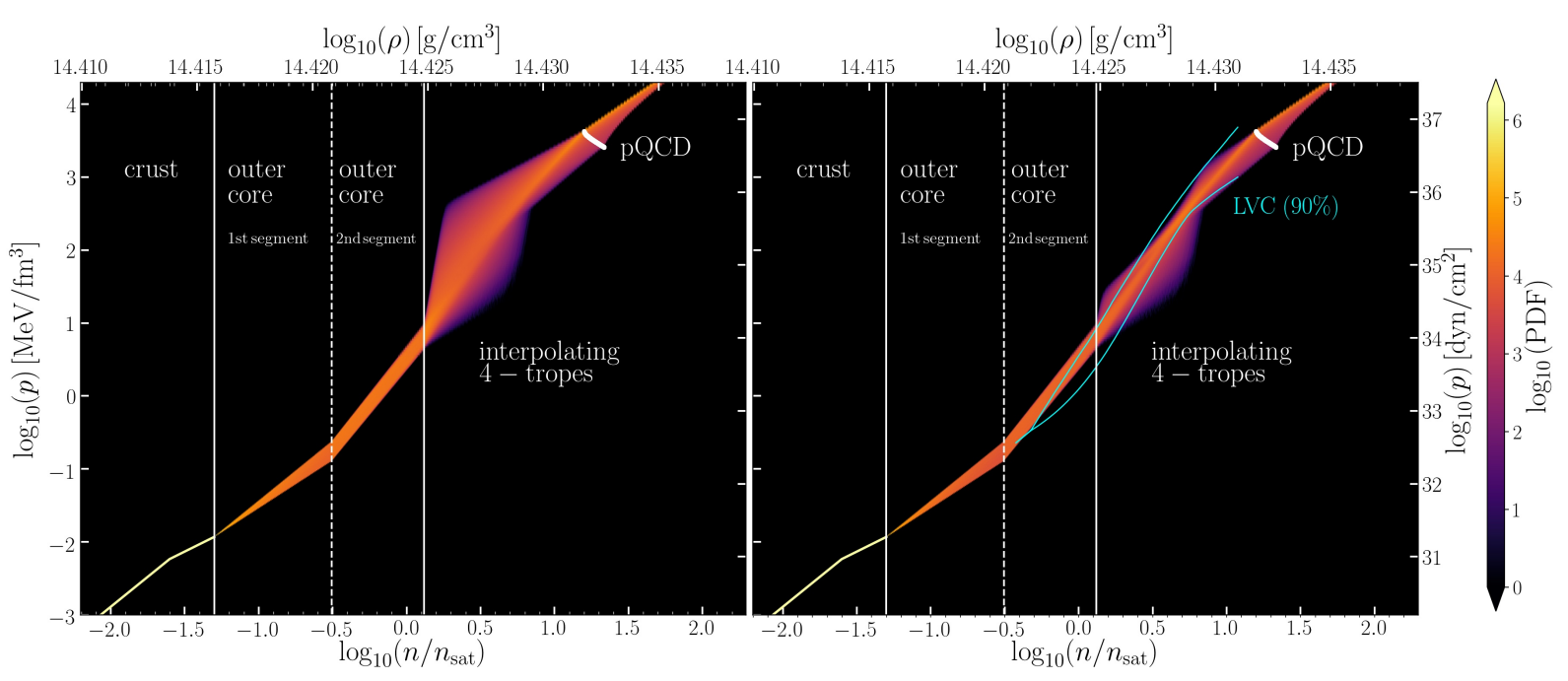

Figure 3.11: Same as Fig. 3.1, but comparing the allowed space for the EOS before (left panel) and after (right panel) GW170817. The right panel also shows the constraints derived by LVC (Abbott et al., 2018) as blue lines for comparison.

is in contradiction with the observed Galactic populations of neutron stars (Farrow et al., 2019) and thus requires to reconsider current models for neutron star formation (Romero-Shaw et al., 2020; Safarzadeh et al., 2020). Unfortunately, no electromagnetic counterpart could be connected to GW190425 and also the tidal deformability did not provide any new information. Such heavy mergers are still useful and could in principle be used to derive a new lower limit on $M_{\mathrm{TOV}}$ as shown in Most et al. (2020c). In this context, also new observations with direct mass measurements have taken place providing a potentially more constraining lower limit than the current value of $M_{\mathrm{TOV}}>2.01$. Unfortunately, this observation (Cromartie et al., 2020) has a large error so that one should take caution when applying the associated value for $M_{\mathrm{TOV}}$ as a constraint.

Recently, the merger GW190814 (The LIGO Scientific Collaboration et al., 2020) with a highly unequal mass ratio was observed, whose lighter component is either the lightest black hole or the heaviest neutron star known to date. A large number of publications has already appeared on this (see e.g., Tan et al., 2020; Essick \& Landry, 2020; Zhang \& Li, 2020; Fattoyev et al., 2020; Godzieba et al., 2020; Sedrakian et al., 2020; Dexheimer et al., 2020). Most notably, in Most et al. (2020b) we could derive a new lower limit on the maximum mass of non-rotating neutron stars, i.e., $M_{\text {то }}>2.08_{-0.04}^{+0.04}$. This limit is derived using universal relations and assuming that the secondary was at some point before merger a rapidly rotating neutron star. The value is only slightly more constraining than the one from direct pulsar observations.

The other major event that took place only two years after GW170817 was the direct observation of pulsars providing radius measurements with unprecedented accuracy. Unfortunately, no additional constraints could be obtained. Nevertheless, such observations complement and strengthen the current constraints and highlight the fact that we truly live in a golden age of astrophysics, where multimessenger observation carried through different fundamental forces (gravitational and electromagnetic force) are possible. 


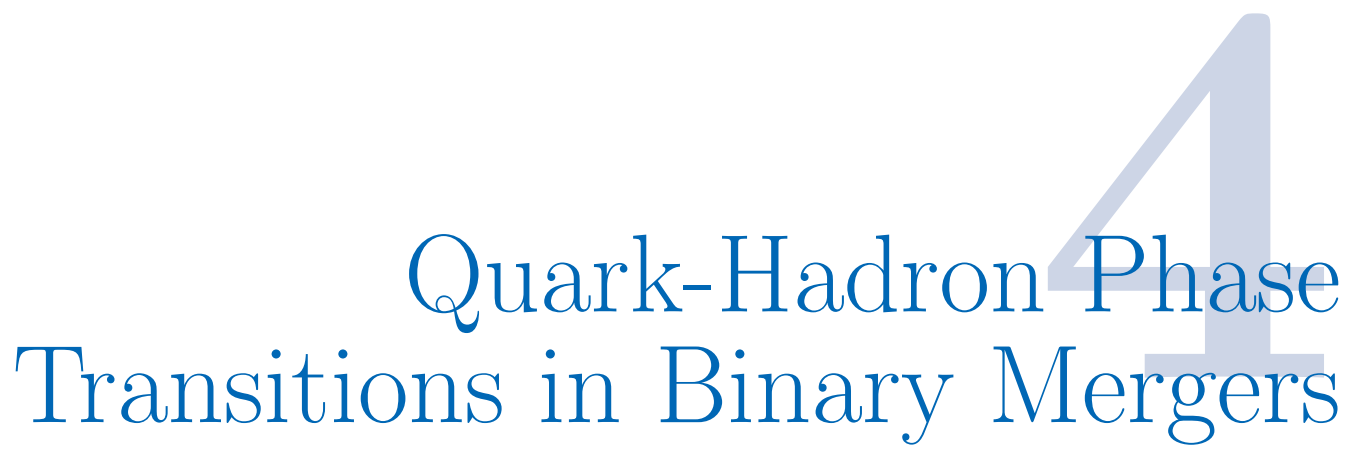

In the previous chapter, we summarized what was learned from GW170817. At this point, it should be recalled that all this information is extracted from the electromagnetic counterpart and the low-frequency inspiral signal alone. Indeed, current gravitational-wave detectors are only sensitive to signals with a frequency below the kilohertz range (Dietrich et al., 2020b). Given that no electromagnetic counterpart could be observed for any detection other than GW170817, the inspiral signal presents the only source of information for all other detections so far. The high-frequency signal from a merger's violent postmerger phase, on the other hand, is yet to be detected. Just like numerical simulations helped to understand and interpret the inspiral signal, they should also be used to prepare for future measurements of the postmerger signal. Indeed, a lot of work has already been done for simulating this signal and connecting it to the EOS. What has been long missing, however, are realistic simulations of binary neutron stars that allow for a phase transition from hadronic to deconfined quark matter during the postmerger phase, where the most extreme thermodynamic conditions are met. This has only been accomplished recently (Most et al., 2019a; Bauswein et al., 2019). In this chapter we focus on the scenario simulated in Weih et al. (2020b); Hanauske et al. (2021), which completes the picture of possible gravitational-wave signals from such phase transitions. First, the different scenarios for producing a phase transition during a binary merger and their corresponding gravitational-wave signature is reviewed in Sec. 4.1, before the delayed-phase-transition case, during which a stable hypermassive hybrid star is produced, is presented and analyzed in detail in Sec. 4.4. The Author's contributions to Weih et al. (2020b); Hanauske et al. (2021) are detailed in Sec. 1.3.

\subsection{The Postmerger Gravitational-Wave Signal}

In contrast to the inspiral, the postmerger phase is much more complicated and can only be modeled via numerical simulations. During this phase the thermodynamic conditions are also the most extreme requiring a number of physical effects to be taken into account, such as radiative neutrino transport, turbulence, heating due to shocks, and the possibility of a phase transition. The probably most important parameter governing this violent postmerger phase is the system's total mass. As already discussed in Sec. 1.2 a high mass will lead to a prompt collapse leaving behind a black 


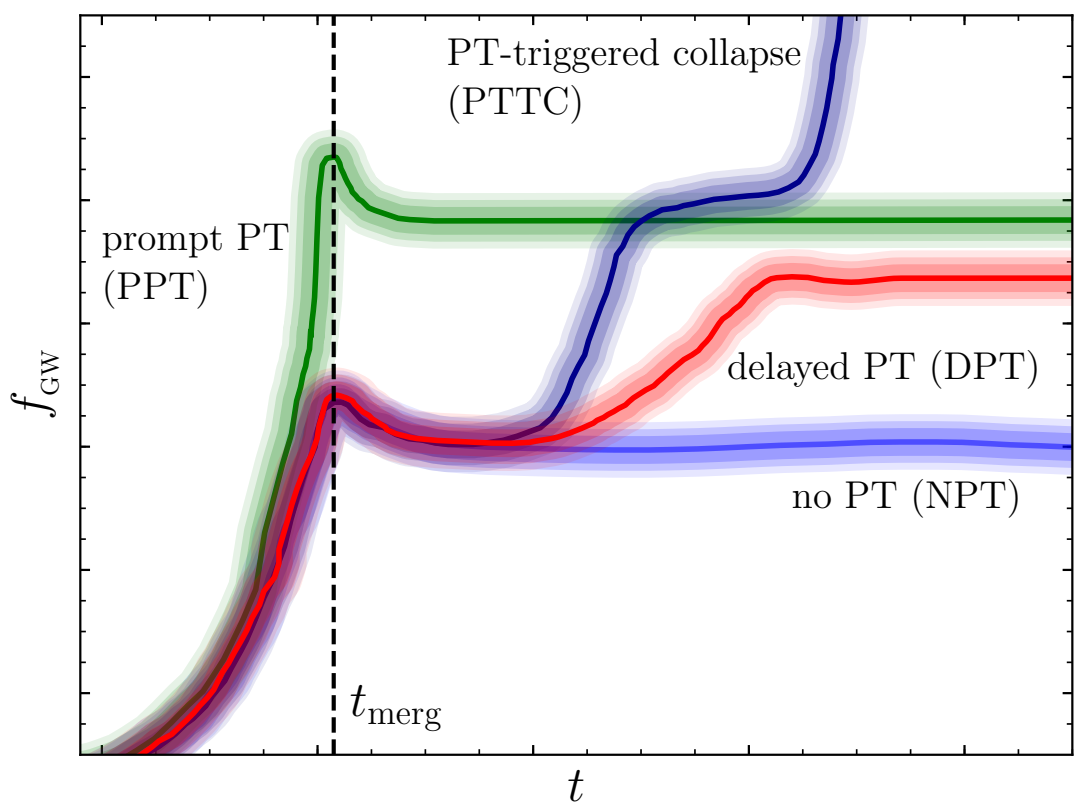

FIGURE 4.1: Schematic overview of the instantaneous gravitational-wave frequency over time for different scenarios of a binary neutron star merger. The vertical dashed line marks the point of merger. The signal during the postmerger phase, i.e., to the right of this dashed line, differs for the four different cases described in the main text. Reprinted from Weih et al. (2020b) with permission by the American Physical Society.

hole-torus system, while a mass below a certain threshold will result in a hypermassive neutron star (HMNS) ${ }^{1}$. The final result of the HMNS again depends sensitively on its total mass, mass ratio, magnetic field evolution, and on the underlying EOS (see also Fig. 1.1 in Sec. 1.2).

The latter contains as a degree of freedom the possibility of a phase transition (PT). Specifically, it is speculated whether a PT from purely hadronic to quark matter might be realized in the dense core of a neutron star (Alvarez-Castillo et al., 2016; Montaña et al., 2019; Alvarez-Castillo et al., 2019; Ayriyan et al., 2018; Christian et al., 2019; Annala et al., 2020). An ideal environment for such a PT to take place would be during the merger of two neutron stars. The high densities and hot temperatures (Perego et al., 2019) reached during the postmerger phase might trigger the PT, which then drastically alters the subsequent evolution. Naturally, the question arises whether this PT could be detected, e.g., via gravitational waves. To answer this question we begin by categorizing the different scenarios in which a PT can manifest (Weih et al., 2020b). We do so by looking at Fig. 4.1, which shows schematically the instantaneous gravitational-wave frequency, $f_{\mathrm{GW}}$, that can be obtained from numerical simulations as outlined in Sec. 2.1.3.

In general, four different cases can be identified:

\footnotetext{
${ }^{1}$ Note that the term HMNS is defined by a star with a mass above the maximum mass of a uniformly rotating neutron star, $M_{\max }$. In Fig. 3.3 this corresponds to the dark-red shaded area. In the literature, however, an HMNS usually refers to any differentially rotating remnant following the merger of two neutron stars. Even if such a remnant has a mass below $M_{\max }$, it is often still referred to as HMNS. In the picture of Fig. 3.3 this is equivalent to describing the entire red-shaded area as HMNS. Even inside the green area it is possible to find differentially rotating neutron stars that - according to the definition - are not HMNSs, but might incorrectly be called like that in the literature. In this chapter we will follow this misleading nomenclature and denote any differentially rotating remnant, independent of its mass, as HMNS for simplicity and trust that the reader keeps the aforementioned clarification in mind.
} 
No phase transition (NPT; light-blue line): This is the most commonly studied and best understood scenario (see e.g., Baiotti \& Rezzolla, 2017; Paschalidis, 2017; Dietrich et al., 2020b, for recent reviews and also Sec. 1.2). No PT sets in after the merger and the matter is purely hadronic during the entire evolution. After the inspiral phase $f_{\mathrm{GW}}$ slightly drops and then reaches a constant frequency produced by the $\ell=2=m$ mode of the merger remnant. Information on the EOS can be inferred via gravitational-wave "spectroscopy": the dominant frequency is connected to the EOS via a quasi-universal relation (Bauswein \& Janka, 2012b; Takami et al., 2014, 2015; Bernuzzi et al., 2015; Rezzolla \& Takami, 2016), so that identifying the peak in the Fourier-transform of $f_{\mathrm{GW}}$ can provide constraints on the EOS.

Prompt phase transition (PPT; green line): The PT sets in during the merger, when the density reaches a maximum. Since the PT manifests itself as a prompt softening of the EOS, the remnant's core becomes more compact so that the gravitational-wave frequency during merger is increased beyond the one reached in the NPT case. The postmerger phase then looks similar to the NPT case with $f_{\mathrm{GW}}$ reaching a constant value, only that this value is at a much higher frequency as is the case in the NPT scenario. This PPT case was first simulated in Bauswein et al. (2019) using a temperature dependent EOS that features a strong first-order PT (Fischer et al., 2018). It was shown that the high frequency in the postmerger phase does not follow the quasi-universal relations known for the NPT case, thus providing a way of identifying the PT.

Phase-transition triggered collapse (PTTC; dark-blue line): After the inspiral the PT sets in either directly at merger (as in the PPT scenario) or delayed during the postmerger phase (as in the DPT scenario). In any case, the softening due to the PT is so strong that a collapse to a black hole is triggered. This has been simulated in Most et al. (2019a) using a temperature dependent EOS derived from a chiral mean field model (Papazoglou et al., 1999; Dexheimer \& Schramm, 2008, 2010), which represents the first fully general-relativistic simulation with quarks at finite temperature. It was shown that in this scenario the PT causes a dephasing of the postmerger signal similar to what was found in Oechslin et al. (2004).

Delayed phase transition (DPT; light-red line): The PT sets in during the postmerger phase, but not immediately after merger. This delayed PT is caused by the continuous increase of the remnant's central density up until the threshold for the transition. The gravitational-wave signal is identical to that of the NPT case until the PT sets in causing the frequency of the signal to increase. Other than the PTTC case, the PT results in a stable hypermassive hybrid star (HMHS), whose subsequent evolution is similar to that of the PPT scenario. Specifically, the HMHS is either secularly stable and eventually results in a uniformly rotating (or even non-rotating) HMHS or it eventually collapses to a black hole.

In the remainder of this chapter we will focus on the DPT case, which has first been simulated in Weih et al. (2020b).

\subsection{Simulation Setup}

The realization of the above scenarios is tightly connected to the characteristic massradius curve of the EOS. This is visualized in Fig. 4.2 for exemplary EOSs. EOS-I 


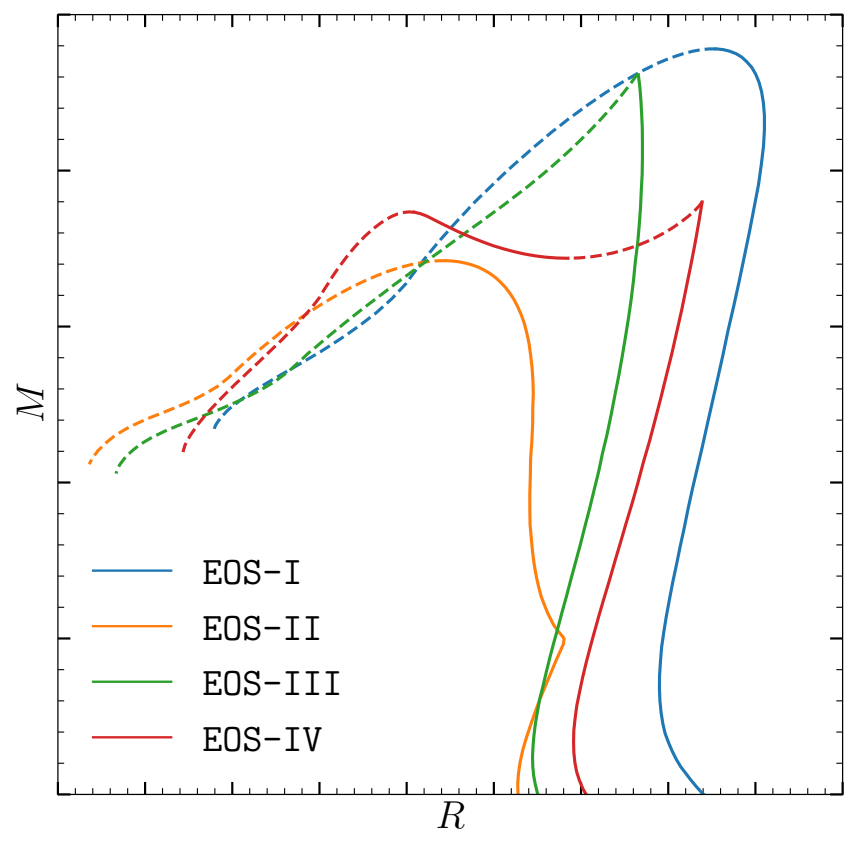

FIGURE 4.2: Schematic overview of mass-radius curves for four EOSs with different types of PTs. A kink in the curve marks the transition from purely hadronic neutron stars to hybrid stars. Solid lines correspond to the stable branches and dashed lines to unstable models. The EOSs and corresponding models are taken from Weih et al. (2019b).

is the standard case and a binary simulated with this EOS would result in the NPT scenario. EOS-II, on the other hand, includes a PT, which is evident from the kink marking the transition from purely hadronic models to hybrid stars. Since the models are not separated by an unstable branch, such an EOS would result in a PPT. The PTTC scenario is realized when using an EOS such as EOS-III, where the PT causes the stable hadronic branch to stop abruptly and all hybrid models are located on an unstable branch. Only EOSs that result in twin stars, as seen for EOS-IV, can produce a DPT scenario. Note that this is only a rule of thumb that helps to understand how these different scenarios are realized. In reality, there is no guarantee for a twin-star EOS to result in a stable HMHS as merger remnant, because the curves shown in Fig. 4.2 show idealized non-rotating equilibrium models.

We therefore use an EOS whose PT is artificially fine-tuned so that twin stars can be obtained. Specifically, for the hadronic phase we use the relativistic mean-field model FSU2H (Tolos et al., 2017a,b). We approximate this EOS with a piece-wise polytropic representation. It is then easy to include a first-order PT from hadronic to quark matter by adding a flat segment. More precisely, we assume a moderate value for the surface tension of deconfined quark matter droplets within the mixed phase, which at zero temperature leads to hadron-quark pasta phase structures (Weber et al., 2019) following the Gibbs condition (Glendenning, 1992). In the cold piece-wise EOS this segment starts at $2.085 \rho_{\text {sat }}$ with a polytropic index $\Gamma=1.04$ and ends at $4.072 \rho_{\text {sat }}$. For the pure quark phase we then match another segment with $\Gamma=5.1$. Three additional segments are used in order to ensure causality throughout the entire EOS. The segments with polytropic indexes $\Gamma=4.7,4.1$, and 3.1 are matched at $4.823,4.969$ and $5.289 \rho_{\text {sat }}$, respectively. From here on, this EOS is denoted as FSU2HPT. The overall cold EOS is shown in the left panel of Fig. 4.3 and the resulting mass-radius curve in the right panel of the same figure. It can be seen that the FSU2H-PT produces a small twin branch, which is formally of Category 3 according 

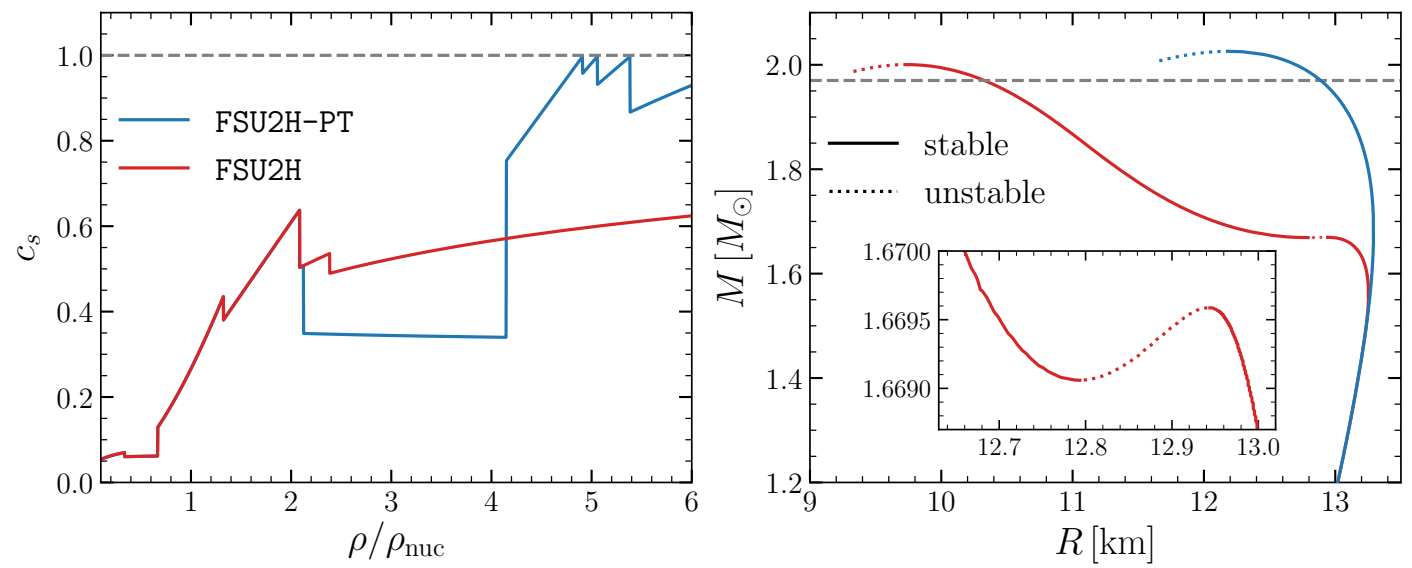

FiguRE 4.3: Sound speed as a function of density (left) and mass-radius curves (right) for the FSU2H (purely hadronic) and FSU2H-PT EOS. The dashed line in the left panel marks the limit $c_{s}=1$ and in the right panel the constraint of $1.97 M_{\odot}$ from pulsar observations. Adapted from Weih et al. (2020b) with permission by the American Physical Society.

to the classification in Christian et al. (2018).

This EOS is only valid at zero temperature. Unfortunately, a self-consistent finitetemperature EOS that leads to the DPT scenario is not available. We therefore make use of the cold EOS and account for heating effects via a hybrid approach that is commonly used in numerical simulations of binary neutron stars (for example in Baiotti et al., 2008; Kiuchi et al., 2014; Hanauske et al., 2017a; De Pietri et al., 2016; Alford et al., 2018; Endrizzi et al., 2016; Radice et al., 2018a). Within this approach an ideal-fluid component accounting for the temperature is added to the cold EOS. The pressure, $p$, and specific energy, $\epsilon$, are then composed of the cold contribution and a thermal one, i.e.,

$$
p=p_{\mathrm{c}}+p_{\mathrm{th}} \quad \text { and } \quad \epsilon=\epsilon_{\mathrm{c}}+\epsilon_{\mathrm{th}} .
$$

The thermal part follows the ideal-gas law, i.e.,

$$
p_{\mathrm{th}}=\rho \epsilon_{\mathrm{th}}\left(\Gamma_{\mathrm{th}}-1\right)
$$

with the thermal adiabatic index chosen as $\Gamma_{\text {th }}=1.75$, which has been found to be a good approximation when comparing to simulations with self-consistent finitetemperature EOSs (Bauswein et al., 2010; Figura et al., 2020). Within this prescription a temperature can be obtained during the simulation according to

$$
T=\frac{m_{\mathrm{n}} p_{\mathrm{th}}}{k_{\mathrm{B}} \rho},
$$

where $m_{\mathrm{n}}$ is the nuclear mass and $k_{\mathrm{B}}$ is the Boltzmann constant.

The simulation is performed by solving the coupled Einstein-hydrodynamic equations. For this we use the framework of the Einstein Toolkit (Loeffler et al., 2012), which implements different physics modules as thorns. The equations of hydrodynamics are solved in the Valencia formulation, i.e., written as Eq. (2.61), by the thorn WhiskyTHC (Radice \& Rezzolla, 2012; Radice et al., 2014a,b). WhiskyTHC uses a high-order finite-differencing scheme reconstructing the variables at cell interfaces via the MP5 reconstruction scheme (Suresh \& Huynh, 1997) and a positivity-preserving limiter (Rezzolla \& Zanotti, 2013). The Riemann problem is then solved via the approximate HLLE solver [see Eq. (2.74)]. 


\begin{tabular}{|l|c|c|c|r|}
\hline Identifier & EOS & $M\left[M_{\odot}\right]$ & Resolution $[\mathrm{m}]$ & Outcome \\
\hline PT-264-hi & FSU2H-PT & 2.64 & 189 & DPT \\
\hline PT-264 & FSU2H-PT & 2.64 & 237 & DPT \\
\hline PT-264-1o & FSU2H-PT & 2.64 & 369 & DPT \\
\hline NPT-264 & FSU2H & 2.64 & 237 & NPT \\
\hline PT-268 & FSU2H-PT & 2.68 & 237 & PTTC \\
\hline NPT-268 & FSU2H & 2.68 & 237 & NPT \\
\hline
\end{tabular}

TABLE 4.1: Summary of binary neutron star simulations within this chapter. Note that all binaries have a mass ratio of $q=1$.

The gravitational field is evolved in the CCZ4 formalism [see Eqs. (2.29)-(2.34)] by the thorn McLachlan (Brown et al., 2009) with the damping parameters $\kappa_{1}=0.06$, $\kappa_{2}=0$ and $\kappa_{3}=0.5$. As gauge conditions we use the ones outlined in Sec. 2.1.2, i.e., $1+\log$ slicing for the lapse and a Gamma-driver with a damping constant $\eta=0.5$ for the shift.

The simulation is performed on a grid with a total size of $\sim 1500 \mathrm{~km}$. This grid is handled by the thorn Carpet (Schnetter et al., 2004), which implements fixed boxin-box mesh refinement. We make use of fixed refinement levels centered around the two neutron stars with the finest level having a resolution of $\mathrm{d} x=0.16 M_{\odot} \simeq 237 \mathrm{~m}$.

Finally, the initial data for the simulations is computed by Lorene (Gourgoulhon et al., 2001), a code that implements multi-domain spectral methods for solving among other things elliptic equations required for initial data computation. We set up irrotational binaries with a mass ratio of $q=1$, an initial separation of $\sim 45 \mathrm{~km}$, and a total binary mass of $M=2.64 M_{\odot}$ and $M=2.68 M_{\odot}$. This setup corresponds to inspiraling stars with initial radii of 13.11 and $13.13 \mathrm{~km}$, respectively. As summarized in Tab. 4.1 we evolve four different systems, i.e., two masses for two EOSs. Since we run one of them with three resolutions in order to check our results for convergence, the total is six simulations.

\subsection{Overall Simulation Results}

The overall dynamics of the different runs can best be summarized when looking at the evolution of the maximum rest-mass density, $\rho_{\max }$, shown in the left panel in Fig. 4.4. Note that during all simulations the point of maximum density is always in the center of either the inspiral neutron stars or of the remnant. For the two runs with the purely hadronic FSU2H EOS (light red and blue lines in Fig. 4.4) the classical dynamics can be observed: during the inspiral (green shaded region) the two stars get closer and the maximum density slightly oscillates due to the tidal forces (this can not be seen in the figure due to its scale). Right before merger, the maximum density decreases and then reaches a local maximum at merger quickly dropping to the density of the inspiral again. Now the violent postmerger phase begins and strong oscillations can be seen. Overall the density of the remnant increases and the oscillations are damped until a meta-stable equilibrium is reached after $\sim 15 \mathrm{~ms}$. The matter is purely hadronic at all times since the EOS does not allow for quark matter ${ }^{2}$.

The two cases with PT (dark blue and red lines) show the same dynamics for the inspiral since here the densities are still within the purely hadronic phase, where the FSU2H-PT EOS is the same as FSU2H. At merger, however, the densities in the

\footnotetext{
${ }^{2}$ Note that while the FSU2H EOS is purely hadronic it is not purely nucleonic since it does include hyperons for densities beyond $\sim 2 \rho_{\text {sat }}$.
} 


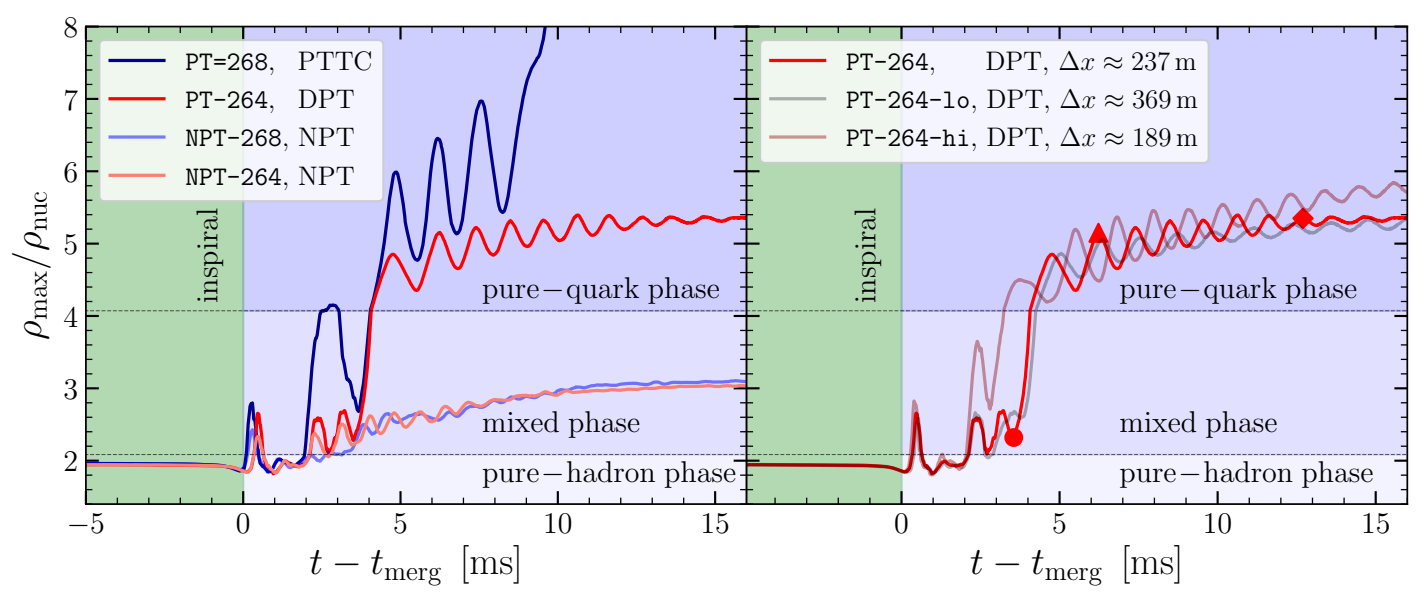

Figure 4.4: Left: Evolution of the maximum rest-mass density for the four runs listed in Tab. 4.1. The inspiral phase is shaded in green and the different phases of the FSU2H-PT EOS in different shades of blue. Note that these phases apply only to PT-268 and PT-264; the NPT-runs are always purely hadronic. Right: Same as the left panel but for PT-264hi, PT-264 and PT-264-1o for showing convergence. The different symbols (circle, triangle, diamond) mark specific times for PT-264 that are discussed in details in Sec. 4.4.1. The left panel is reprinted from Weih et al. (2020b) with permission by the American Physical Society.

core reach a value above the threshold for the mixed phase. At this point the first quarks appear. The increase of the density is mostly due to the compression of the stellar cores. It is evident that the softening of the EOS in the mixed phase slightly contributes to this compression and indeed the density peaks at merger for models PT-264 and PT-268 are slightly larger than the ones for NPT-264 and NPT268. Nevertheless, this increase is not enough to cause the PPT scenario and the densities quickly fall back below the threshold of the mixed phase and the matter is again purely hadronic. As is the case for NPT-264 and NPT-268 the violent postmerger phase is characterized by density oscillations and a gradual overall compression of the remnant. This compression causes the central densities again to exceed the threshold of the mixed phase at which point the density oscillations are drastically increased due to the strong softening of the EOS.

The subsequent evolution now depends on the total binary mass and we first focus on the heavier system, PT-268 (dark-blue line), and leave the more interesting case, i.e., PT-264 (dark-red line), to be the focus of the next section. For PT-268 the center even enters the pure quark phase $\left(\rho \gtrsim 4.1 \rho_{\text {sat }}\right)$, but quickly falls back again in the mixed phase. Since the overall density is increasing during the oscillations, the next oscillation already reaches densities high enough so that the entire core remains in the pure quark phase. At this point $\sim 20 \%$ of the hadrons have been converted into quarks, which is in agreement with what was found in Most et al. (2020a); Bauswein et al. (2019). The EOS at these high central densities $\left(\sim 5-7 \rho_{\text {sat }}\right)$ is now extremely stiff (see also the left panel in Fig. 4.3) making it difficult to further compress the star. Due to the previous rapid increase in density large oscillations occur regardless. In the end, the heavy system's gravity prevails and causes the remnant to collapse to a black hole quickly after the PT sets in. Since the quark core only survives a few milliseconds and is doomed to collapse from the moment the PT sets in, this case can clearly be characterized as PTTC. 


\subsection{The Delayed-Phase-Transition Scenario}

The binary with the lower mass of $M=2.64 M_{\odot}$, i.e., PT-264 has a similar evolution as PT-268 until $\sim 3 \mathrm{~ms}$ after merger. After this point, the central density again increases drastically due to the softening of the EOS. However, this time the subsequent stiffening within the pure quark phase is sufficient to halt the collapse and instead a metastable HMHS is produced. Since this transition happens not directly at merger, but only delayed after an HMNS is formed first, this scenario can clearly be classified as DPT. This simulation presents the first time that a DPT scenario could successfully by simulated. Therefore, the remainder of this chapter will be focused on a detailed analysis of this simulation.

\subsubsection{Thermodynamic Properties of a Hypermassive Hy- brid Star}

As evident from Fig. 4.4, the merger and the following postmerger phase is initially the same in the DPT case as discussed for the PTTC and the NPT cases. At $\sim 3.5 \mathrm{~ms}$ (see red circle in the right panel of Fig. 4.4) a few quarks have been produced inside the remnant's core. The left column of Fig. 4.5 shows from top to bottom the restmass density, $\rho$, temperature, $T$, and matter distribution in the $T-\rho$ phase space, respectively. It can be seen that the quarks are contained in two circular regions slightly off-center (see cyan colored lines). These are the region of highest density and coldest temperature. Such a "peanut" shape is typical also for mergers without PT and arises from the former cores of the two component neutron stars. It is also evident, that this peanut is slightly asymmetric.

This small asymmetry and the double-core structure is amplified during the subsequent collapse. Consequently, right after this partial collapse (red triangle in Fig. 4.4) the resulting HMHS has a sizable one-sided asymmetry. In terms of a decomposition with a spherical-harmonics basis this corresponds to an $m=1$ mode. The amplification of this asymmetry is also the reason why different grid resolutions have a visible impact in our simulations as can be seen from the right panel in Fig. 4.4: the transparent lines show the same DPT run at a higher and smaller resolution. Evidently, the overall result and most importantly the realization of the DPT scenario is a convergent behavior of our simulations. The oscillations, however, differ significantly with resolution (this is well visible for PT-264-hi). Typically the trajectory of the neutron stars already during the inspiral varies with resolution, so that the relative position of the two stars at merger varies and consequently the small asymmetry in the remnant is resolution dependent. In a typical run this effect is barely visible, but due to the amplification caused by the PT these small differences now lead to visible differences in the overall evolution.

The second panel in Fig. 4.5 shows the HMHS following the partial collapse. The previously mentioned $m=1$ mode is now well visible in the top panel. At this point the pure-quark core comprises $\sim 20-30 \%$ of the total binary mass. The middle panel and bottom panel show that two distinct regions exist, where the temperature reaches a local maximum. The outer maximum (and the hotter of the two) with $T \sim 95 \mathrm{MeV}$ is outside of the quark core and again a typical feature that is also present in an NPT merger (Hanauske et al., 2017a; Kastaun et al., 2017) resulting from the matter being shocked during the merger. The inner temperature hot-spot around $T \sim 65 \mathrm{MeV}$ is much smoother. Since this hot-spot falls within the mixed-phase region and is not present in a NPT merger, it can be attributed to the occurrence of quark matter; the 

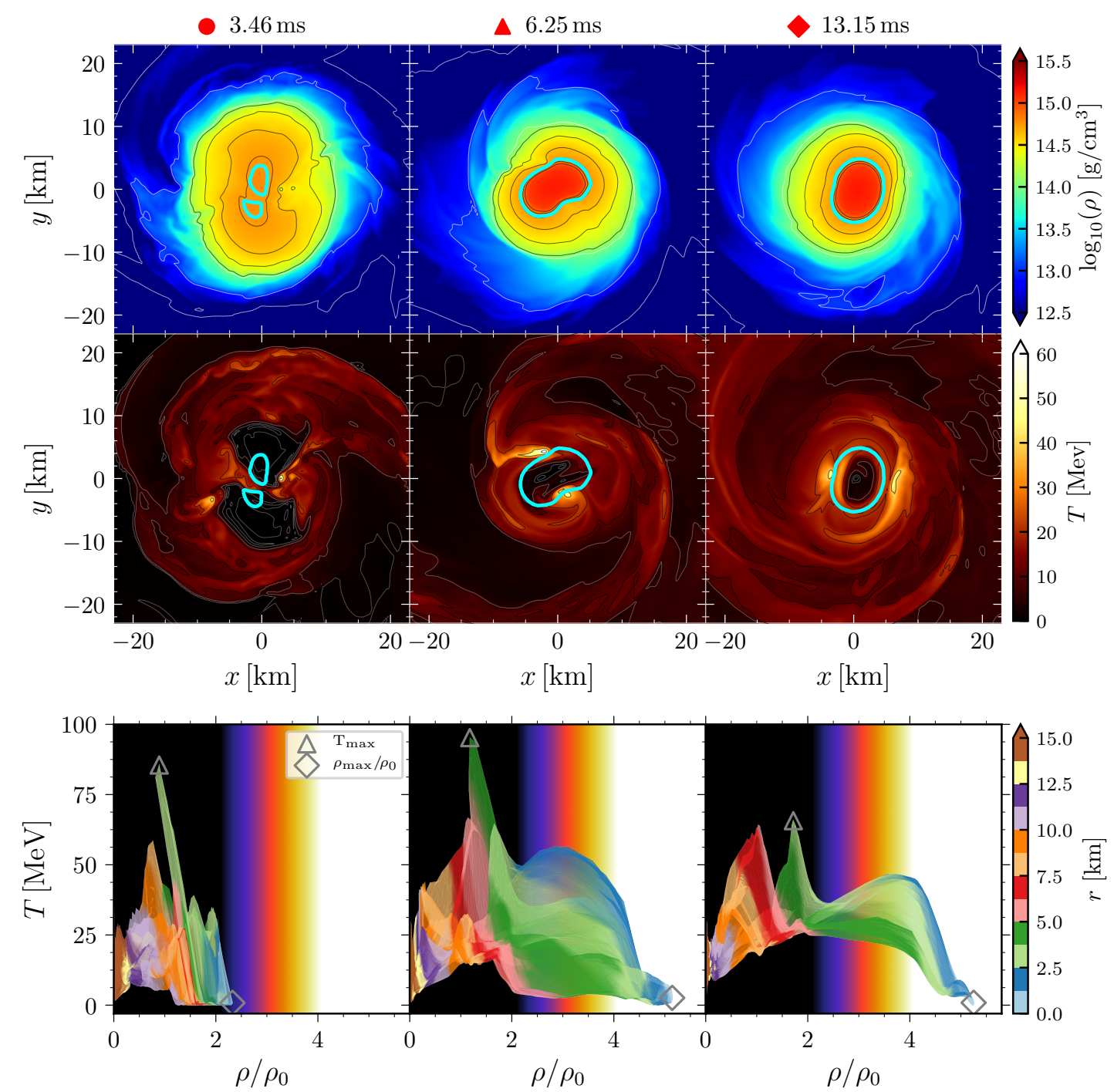

Figure 4.5: Rest-mass density (top), temperature (middle) and matter distribution in a QCD-style phase diagram (bottom) for the three representative times that are marked by different red symbols in the right panel of Fig. 4.4. The cyan lines mark the region within which quark matter exists. In the bottom panel the foreground color encodes the radial position relative to the remnant's center, while the background color shows the quark fraction (black for purely hadronic and white for pure quark matter). Also marked with a triangle and a diamond are the locations of maximum temperature and rest-mass density, respectively, in the bottom panel. Reprinted from Hanauske et al. (2021) with kind permission of the European Physical Journal (EPJ).

additional heating in this region stems from strong shocks within the soft mixed-phase region of the HMHS.

The subsequent evolution is characterized by strong density oscillations. During these oscillations the temperature hot-spots are smeared out and form a ring like structure in the equatorial plane. At $\sim 13 \mathrm{~ms}$ (see right-most panel in Fig. 4.5) the HMHS has reached a meta-stable equilibrium. The corresponding phase-space diagram shows that there is a smooth temperature hot-spot within the pure quark core and two local maxima in the hadronic part of the remnant. The location of these latter two maxima would coincide in a purely hadronic merger, but can be found at different radii for the DPT scenario due to the enhanced asymmetry discussed 


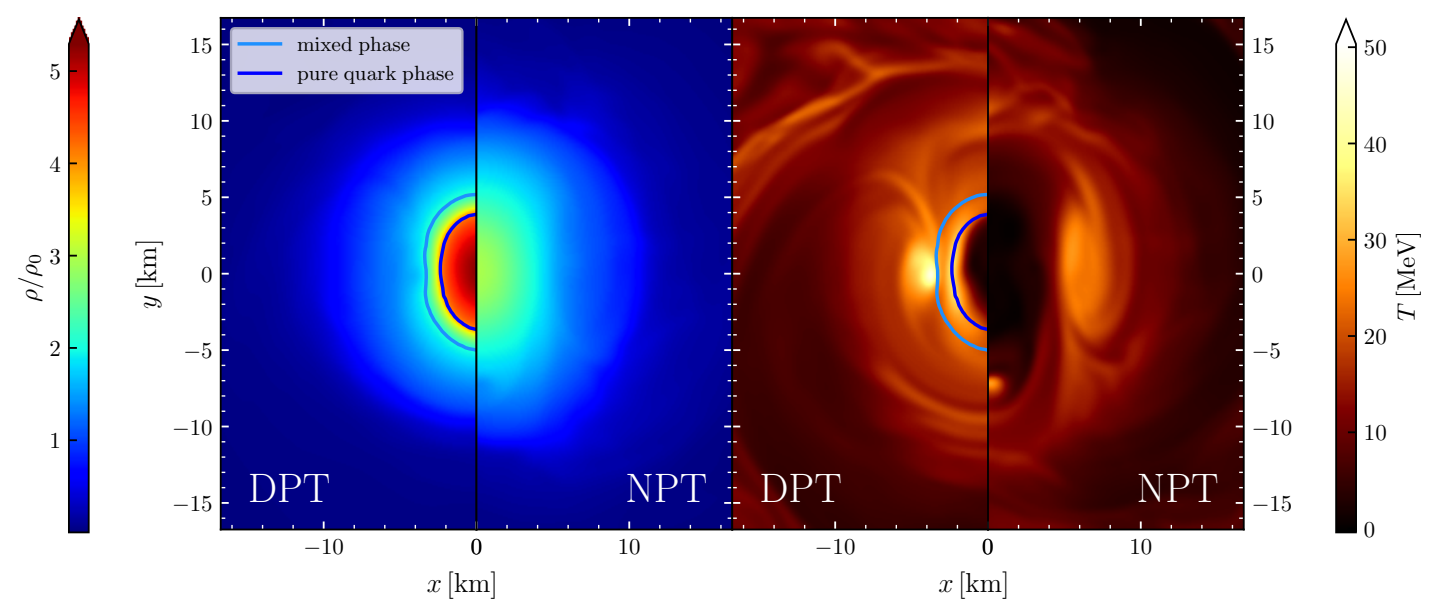

Figure 4.6: Comparison of the rest-mass density (right panels) and the temperature (left panels) for the same runs with and without PT. The left portion of the panels refers to PT264 and the right portion to NPT-264. The snapshot corresponds to the same time as the right-most panels in Fig. 4.5 (diamond in Fig. 4.4). The light and dark blue lines mark the beginning and end, respectively, of the mixed phase region for the case with PT. Reprinted from Weih et al. (2020b) with permission by the American Physical Society.

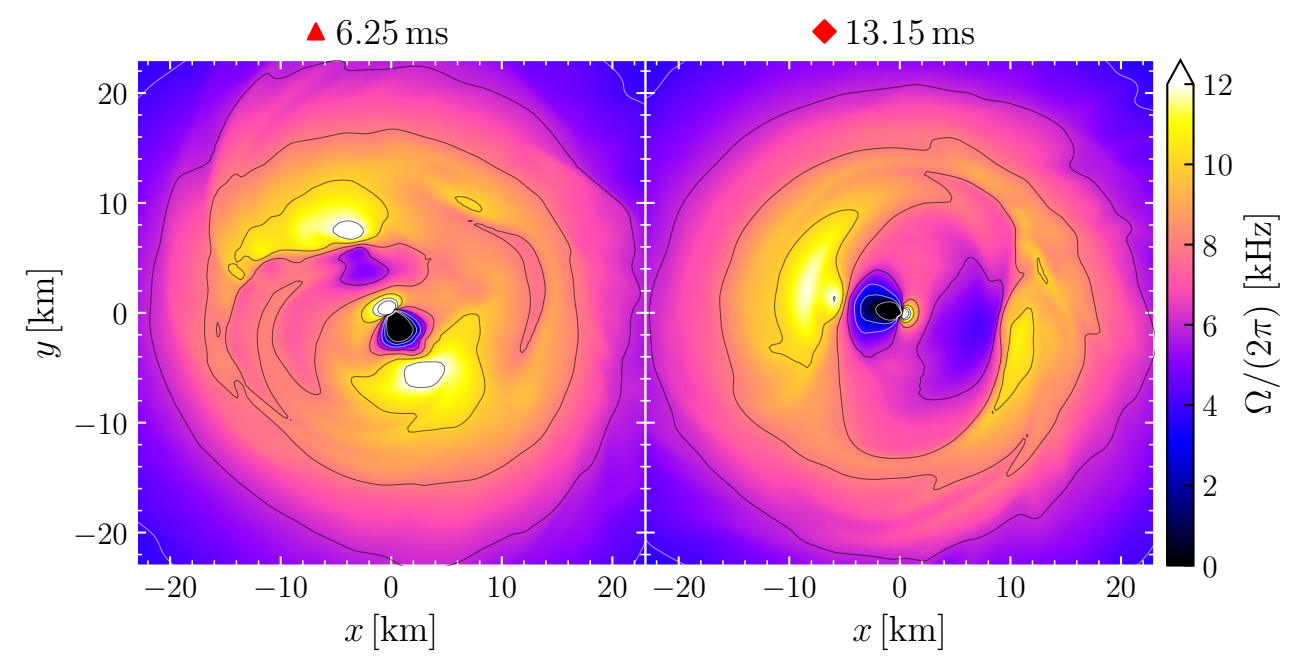

Figure 4.7: Angular velocity, $\Omega$, in the equatorial plane for PT-264 at two representative times (cf., Fig. 4.4). Contour lines are drawn for $\Omega \in[0,2,4] \mathrm{kHz}$ (white) and $\Omega \in[6,8,10,12,14] \mathrm{kHz}$ (black). Reprinted from Hanauske et al. (2021) with kind permission of the European Physical Journal (EPJ).

previously.

We also compare the state of the HMHS at this stage with that of a purely hadronic merger, i.e., with NPT-264, at the same time in Fig. 4.6. The core of the remnant from PT-264 is much denser and in general the temperature is higher compared to the purely hadronic remnant. In particular, the densities are almost twice as large inside the core, which occupies a considerable fraction of the entire remnant. As can be seen from the thin blue lines representing the beginning and end of the mixed phase, this core is surrounded by a thin shell of $\sim 1 \mathrm{~km}$ thickness marking the transition region between the inner quark core and the outer hadronic part.

Finally, we also show the distribution of the angular velocity, $\Omega:=\alpha v^{\phi}-\beta^{\phi}$, in the equatorial plane for two representative times in Fig. 4.7. Typically, the temperature 


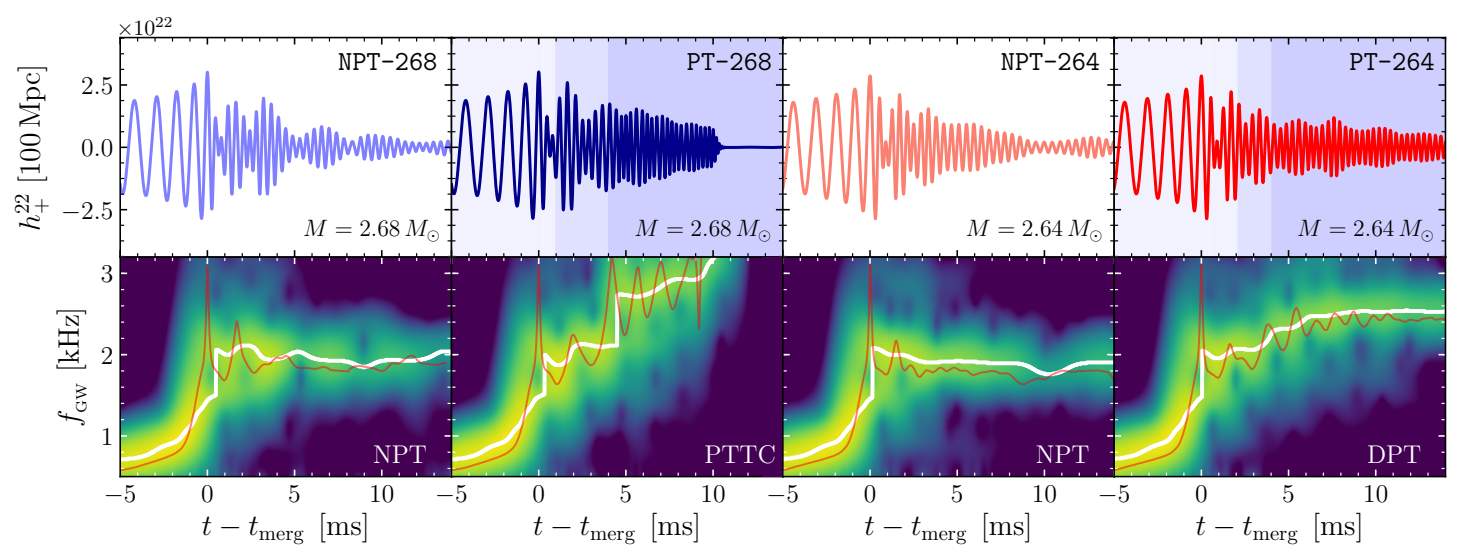

Figure 4.8: Top: Strain, $h_{+}^{22}$, for four of the binary neutron star merger simulations considered in this chapter. The blue shadings mark the times at which the remnant's core enters the different phases (as in Fig. 4.4). Bottom: The color-code shows the spectrograms of the strain in arbitrary units. The white lines trace the maximum of this spectrogram in time. The red lines show the instantaneous frequency. Reprinted from Weih et al. (2020b) with permission by the American Physical Society.

hot-spots are correlated to the differential rotation; the places of maximum temperature are also where the angular velocity reaches a maximum (Kastaun et al., 2016; Hanauske et al., 2017a,b; Kastaun et al., 2017). Comparing to the corresponding temperature panels in Fig. 4.5 reveals that this is also the case for the DPT scenario. Interestingly, the hotter one of the two outer hot-spots corresponds to the smaller one of the two maxima in $\Omega$. This can likely be explained with the fact that the conservation of the Bernoulli constant causes the evolution of $\Omega$ and the density to anticorrelate (Hanauske et al., 2017a). For the inner, smooth ring-shaped temperature maximum inside the pure quark region of the HMHS no such counterpart in $\Omega$ exists, again strengthening the conclusion that this region of increased temperature is caused by the softness of the EOS.

\subsubsection{Gravitational-Wave Signature of a Stable Hypermas- sive Hybrid Star}

The main observable from such a merger is the emitted gravitational-wave signal. In this section is shown how the previously discussed thermodynamic changes throughout the remnant's evolution create such a signal with a distinct signature that allows us to identify the PT.

We start by looking at the strain, which has been introduced in Sec. 2.1.3 [see Eq. (2.50)]. Specifically, we show the plus polarization of the $\ell=2=m$ mode, $h_{+}^{22}$ for all four runs in the top panels of Fig. 4.8. The bottom panels show the spectrograms, i.e., the Fourier transform of the strain at all times, together with the maximum of the power spectral density (white lines) and the instantaneous frequency introduced in Eq. (2.52) (red lines). For the two NPT cases, i.e., NPT-264 and NPT268 , the properties of a standard waveform can be seen. These waveforms have been studied exhaustively in the literature (Bauswein \& Janka, 2012a; Takami et al., 2014, 2015; Bernuzzi et al., 2015; Rezzolla \& Takami, 2016; Maione et al., 2017; Kawaguchi et al., 2018; Dietrich et al., 2019) and their overall form is also described in Sec. 1.2. Here, we are particularly interested in the postmerger signal, the part after the chirping frequency. In the spectrograms both NPT cases show the well known 


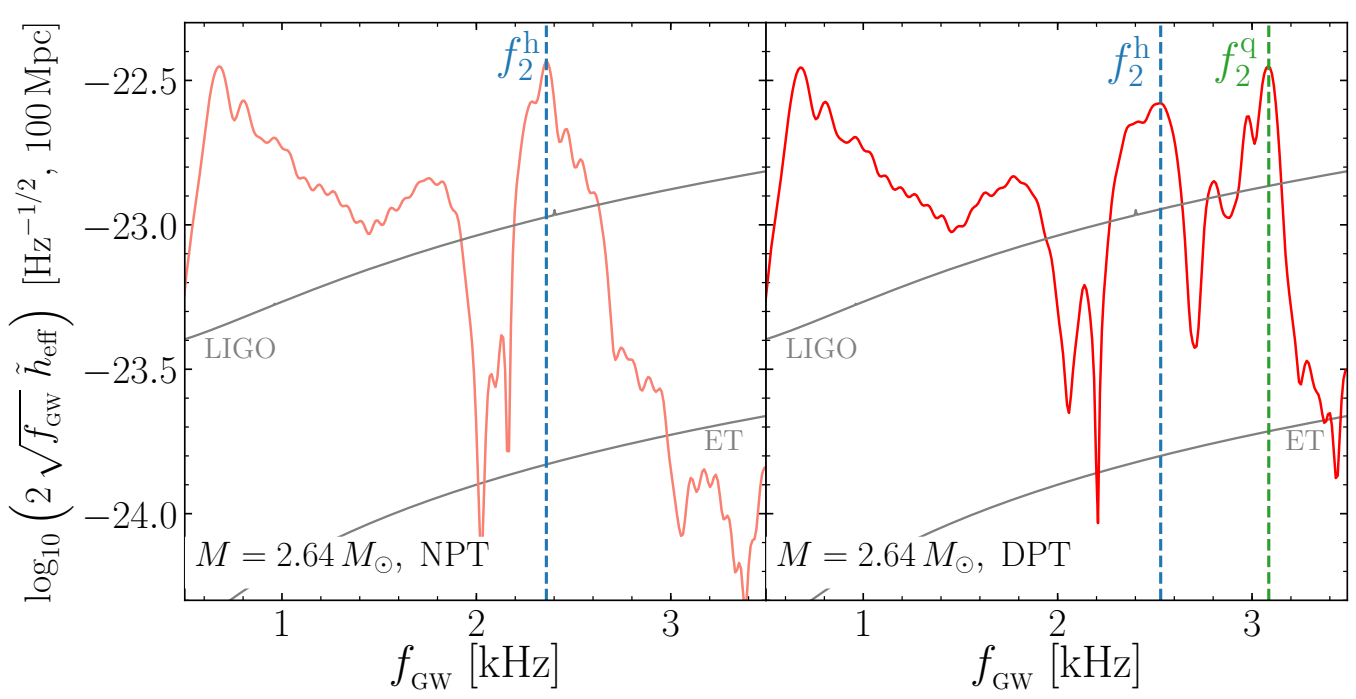

Figure 4.9: Total spectrum for the $l=2=m$ mode of the effective strain [cf., Eq. (2.51)] computed by integrating over both the inspiral and the postmerger phase. The two panels compare the NPT and DPT scenario. The former shows the typical hadronic peak, $f_{2}^{\mathrm{h}}$, with a vertical blue dashed line, while the latter has an additional quark peak, $f_{2}^{\mathrm{q}}$ marked by a vertical green dashed line. The gray lines correspond to the sensitivity curves of advanced LIGO (Barsotti et al., 2020) and ET (Punturo, 2020), respectively. Reprinted from Weih et al. (2020b) with permission by the American Physical Society.

triplet of frequencies right after the merger (Takami et al., 2014), which transitions into a single dominant frequency, $f_{2}$, after $\sim 5 \mathrm{~ms}$.

The PTTC scenario (second column from the left) also shows this behavior. However, after $\sim 5 \mathrm{~ms}$ a sudden jump in the maximum power spectral density is observed. This is exactly at the moment, when the central density increases due to the PT. The compact HMHS that is formed now rotates at a much higher frequency (due to angular momentum conservation). While this is a clear signature of the PT, in reality it would be difficult to detect since only $\sim 5 \mathrm{~ms}$ later the HMHS collapses to a black hole that only emits a weak ringdown signal.

The situation is different for the DPT scenario shown in the right-most panel of Fig. 4.8. Here the increase of the $f_{2}$ frequency during the postmerger phase is less rapid just like the increase in density due to the $\mathrm{PT}$ is smaller compared to the PTTC case. Most importantly, the resulting HMHS is stable and acts as a steady emitter of gravitational waves with this increased frequency over a significant timespan.

The effect of this continued emission becomes evident when looking at the total power spectrum and comparing it to the situation without PT. Fig. 4.9 shows the total power for the case of NPT-264 (left panel) and compares it to PT-264 (right panel). In the left panel on can see again the standard case: going from small to large frequency the power decreases, because the closer the two neutron stars get during the inspiral the less time they spent emitting at a given frequency. Around $f_{\mathrm{GW}} \approx 2.2 \mathrm{kHz}$ a peak (blue dashed line) is observed. This peak is the dominant $f_{2}$ frequency coming from the continued emission of the meta-stable HMNS. In the right panel, i.e., for the DPT scenario, a second peak at a somewhat higher frequency can be seen (green dashed line). This quasistationary frequency, $f_{2}^{\mathrm{q}}$, comes from the more compact and thus more rapidly rotating HMHS and is $\sim 25 \%$ larger than the quasistationary hadronic frequency, $f_{2}^{\mathrm{h}}$. The simple fact that two peaks (instead of just one) emerge in the power spectrum is a clear signature of the PT that led to a meta-stable HMHS. 


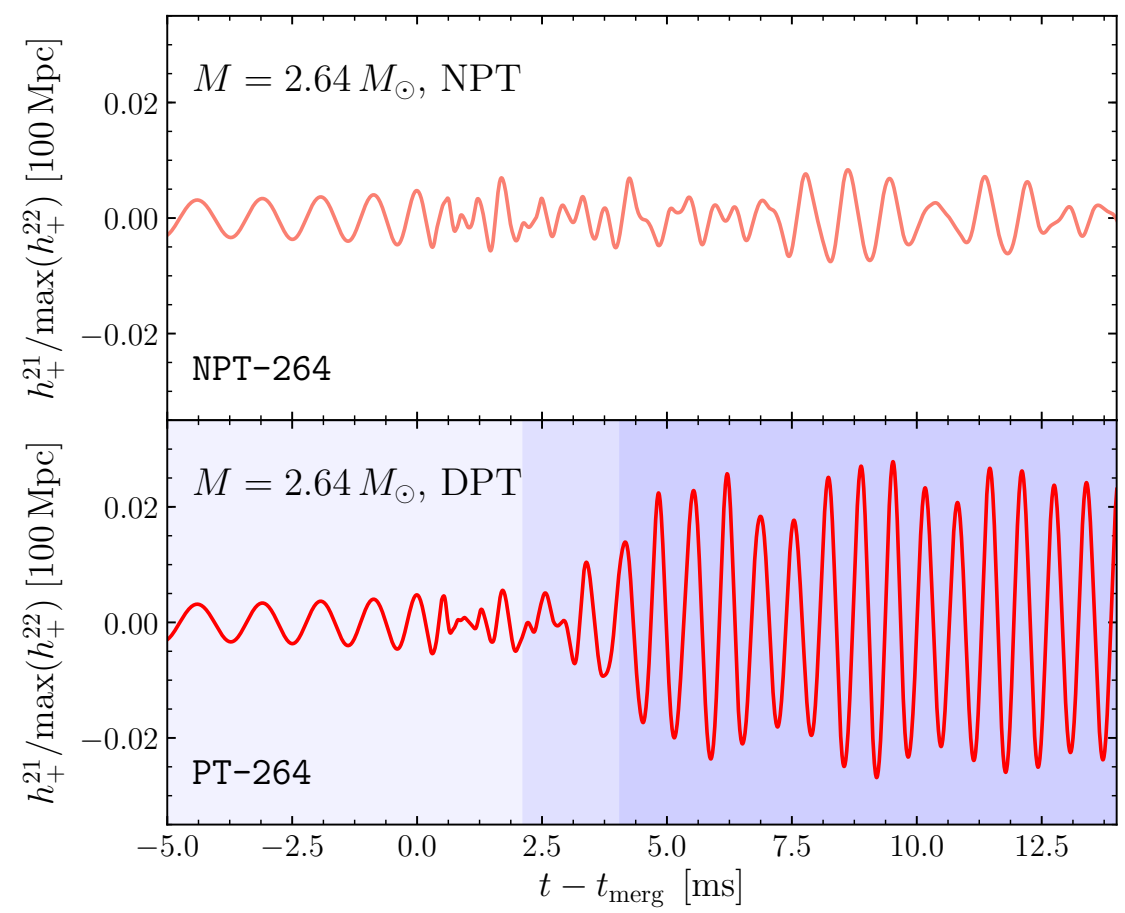

FiguRE 4.10: $\ell=2, m=1$ mode of the plus polarization of the gravitational-wave strain normalized to the maximum amplitude of $h_{+}^{22}$. The top and bottom panels compare the NPT and DPT scenarios. Reprinted from Weih et al. (2020b) with permission by the American Physical Society.

Current gravitational-wave detectors are not sensitive to the frequencies in the $\mathrm{kHz}$ range expected from the postmerger. However, third-generation detectors like advanced LIGO or the Einstein Telescope (ET) should be able to also pick up this signal (Punturo et al., 2010; Abbott \& et al., 2017). Specifically, for the DPT case analyzed here we compute an SNR of 2.43 for advanced LIGO and 6.94 for the ET according to Eq. (2.54). The gray lines in Fig. 4.9 also show that the peaks in the spectrum lie just above the sensitivity curves of these two detectors.

The "double-peak" structure of the total spectrum presents the so far clearest signature of a PT. On top of that, there is also another signature associated to the DPT scenario: the amplified asymmetry in the density distribution following the collapse to the HMHS causes the remnant to rotate in a non-spherical way. This rotation triggers a $\ell=2, m=1$ mode in the gravitational-wave signal. Typically, most of the power is in the $\ell=2=m$ mode and all other modes are negligible. Also in the DPT case most of the power is still in this $\ell=2=m$ mode. However, as is shown in Fig. 4.10 the $\ell=2, m=1$ mode is significantly enhanced in the postmerger phase for the DPT (bottom panel) case compared to the standard NTP scenario (top panel). Although this mode is still at least one order of magnitude smaller than the primary mode, i.e., $\left|h_{+}^{21}\right| /\left|h_{+}^{22}\right| \lesssim 0.1$, and is thus much harder to be detected, it represents an additional signature for the occurrence of a PT in a binary neutron star merger.

\subsection{Summary and Outlook}

In this chapter the possible manifestations of a PT in binary neutron star mergers have been classified based on Weih et al. (2020b). Following the pioneering studies that 
simulated for the first time the PTTC (Most et al., 2019a) and the PPT (Bauswein et al., 2019) scenario, we here completed the picture by presenting the DPT scenario. This scenario is unique in that during the evolution an HMNS as well as an HMHS is produced, i.e., two fundamentally different meta-stable remnants. It was shown that this happens when the PT sets in at a density that is reached only after some time in the postmerger phase. The prompt softening of the EOS leads to a collapse that is then halted by the stiff quark phase. This process results in an HMHS with a large quark core.

It was shown how the specific thermodynamic properties during the DPT evolution cause a distinct signature in the gravitational-wave signal. Specifically, a second peak emerges in the total power of the gravitational-wave strain. It should be noted that in principle these peaks can be very close and - taking into account the error from a potential measurement - they might not be distinguishable. The same would be also true for any other signature. The PPT scenario, for example, gives a single peak that breaks universal relations (Bauswein et al., 2019). Taking into account the error of this quasi-universal relation and the error of the measurement, this frequency might be indistinguishable from a hadronic peak. In both cases an unequivocal signature could only be identified if either the PT is strong or the measurement precise enough. Nevertheless, the DPT signature seems to be the clearest one and easiest to detect since it is a qualitative feature and not a quantitative one in contrast to the signatures of the other classes. While the DPT scenario promises the clearest signature for witnessing the creation of quark matter in our Universe, it should not be misunderstood that it is in any way more or less likely than the other scenarios. Ultimately Nature decides which signature - if any at all - it will present to us.

It should be remarked that the above classification only concerns the postmerger phase. Depending on the binary mass, the mass ratio, the onset and density-jump of the PT, it is also possible to imagine a scenario, in which the PT sets in already during the inspiral. This would provide further manifestations and possible signatures of a PT and is a scenario that should be explored in future studies. Also the DPT scenario itself should be further investigated to see how additional physics like viscous effects, magnetic fields or neutrino transport impact the overall signal. Especially viscosity seems to have a significant impact on the gravitational-wave emission (Radice, 2020).

Finally, numerical simulations can also be used to find out more details about a possible PT; by systematically changing the PT and analyzing the resulting signals, we could find out whether we can not only infer a PT, but even deduce quantitative features like the onset density or the type of PT. A first study in this direction focusing on the PPT scenario has recently been undertaken (Blacker et al., 2020; Bauswein \& Blacker, 2020). 


\section{ort in \\ Radiative Transport in Numerical Astrophysics}

The part of the evolution of a binary neutron star system that is so far the least understood is its long-term evolution. On one hand, this is due to the lack of computational power for fully three dimensional binary neutron star simulations up to an evolved time of seconds, and on the other hand due to the additional physics that starts to play a role during the long-term evolution. One additional part of physics that can easily be neglected for the inspiral and short-term postmerger phase, but not for the long-term evolution, is radiative transport due to neutrinos that are being produced, absorbed and scattered by the hot and dense fluid. These reactions are going to alter the system's evolution in three major ways:

- The production of neutrinos is going to cause a neutrino-driven wind leading to additional ejected material. Indeed, as mentioned in Sec. 1.2 the kilonova signal observed for GW170817 likely consisted of two components, i.e., the dynamically and secularly ejected component, respectively. The large amount of inferred ejecta for GW170817 (Cowperthwaite et al., 2017; Drout et al., 2017) is where the mismatch between observation and simulations is the largest and can likely only be explained with long-term evolutions taking radiative transport into account.

- The composition of the ejected material will also be significantly altered due to the reaction of the neutrinos with the fluid. Charged-current reactions can turn neutrons into protons, thus producing less neutron-rich ejecta. This in turn has consequences for the elements and their abundances that are produced via nucleosynthesis in the ejected material (Rosswog, 2005; Perego et al., 2014; Fujibayashi et al., 2017; Miller et al., 2019a).

- Finally, no simulation has yet shown in a self-consistent way the production of an SGRB. However, from GW170817 and the detection of an SGRB 1.7 seconds later from the same source we now have definite proof that the merger of neutron stars is linked to the production of an SGRB. For successfully simulating this phenomenon the correct treatment of magnetic fields together with radiative transport is believed to be required (Fujibayashi et al., 2017). Neutrinos might help cleaning the polar regions above the HMNS and thus provide a clean funnel through which the jet can break out.

Besides this three-fold importance for binary neutron stars, radiative transport also plays a major role in almost every other high-energy astrophysical phenomenon. Most importantly in supernova simulations, where the production of neutrinos is crucial for the explosion mechanisms. In accretion scenarios, on the other hand, radiation in the 
form of photons instead of neutrinos plays an important role. This final chapter therefore focuses on the numerical treatment of radiative transport.

First, a common method for radiative transport - the so-called M1 method - will be detailed in Sec. 5.1 based on the results of Weih et al. (2020c). After discussing how to implement this method in a general-relativistic framework, the implementation is verified with a number of standard code tests and finally the method is applied to the radiative Michel solution. In the second part of this chapter, i.e., in Sec. 5.2, an entirely new method for radiative transport is introduced. This Lattice Boltzmann method was worked out in Weih et al. (2020a) and promises to be more accurate than the commonly used M1 method at a comparable computational cost. After laying down the basic equations for this method, its implementation is verified with the same code tests as for the M1 method, this time focusing on a comparison with the latter. Finally, this new method is applied to the simulation of relativistic jets proofing its suitability for numerical high-energy astrophysics.

\subsection{The Two-Moment Scheme for General-Re- lativistic Radiation Hydrodynamics}

In Sec. 2.3.2 we briefly introduced a number of alternative methods for solving - or at least approximating - the seven-dimensional Boltzmann equation, which governs the evolution of radiation particles. The M1 method was remarked to be among the most commonly used methods due to its good compromise between feasibility and accuracy. It is used for simulating photons in accretion disks around black holes (Zanotti et al., 2011; Fragile et al., 2012; Roedig et al., 2012; Sądowski et al., 2013; Fragile et al., 2014; McKinney et al., 2014), for driving the explosion mechanism via neutrino emission in core-collapse supernova (O'Connor, 2015; Just et al., 2015b; Kuroda et al., 2016; Skinner et al., 2019) and for binary neutron star mergers (Foucart et al., 2016b; Sekiguchi et al., 2016) or black hole-neutron star mergers (Foucart et al., 2015, 2016a).

As part of this Thesis the M1 scheme has also been implemented within the stand-alone Frankfurt Radiation Code, FRAC (Weih et al., 2020c). This code can easily be coupled to already existing GRMHD codes. In this way, such a code can be extended to perform general-relativistic radiative-transfer magneto-hydrodynamics (GRRTMHD) simulations. FRAC supports evolutions with a fixed spacetime and also for dynamically evolving spacetimes.

In the following three subsections the implementation of the M1 scheme within FRAC will be detailed, verified and applied to a realistic high-energy astrophysical system. It should be noted that many different implementations of the M1 scheme exist in the literature that mainly differ in the following three aspects:

- The closure relation for computing the pressure tensor; depending on the choice for the closure the M1 method can either treat the optically thin, thick or both limits. Sophisticated closures also allow for a reasonable interpolation in the intermediate regime.

- The time evolution method; in general, the M1 equations are a stiff system of equations, i.e., the collisional source term has a numerical value much larger than that of the evolved variables. This requires an implicit time-stepper which, while providing numerical stability, might drastically increase the computational 
complexity of the implementation. Therefore, different levels of approximation can be used for solving the implicit equations.

- The inclusion of a dependence on the frequency of the radiation; while the M1 scheme downscales the angular dependence of the radiation distribution function via the evolution of moments, these moments still have a frequency dependence. Most implementations ignore this dependence and only evolve frequency-averaged moments within a gray scheme. Few implementations include also frequency dependence (O'Connor, 2015; Anninos \& Fragile, 2020) increasing the computational cost linearly by a factor $B$, where $B$ is the number of frequency bins.

For the implementation presented here, we only briefly mention frequency dependence and then focus on a gray implementation and discuss the first two points in detail throughout the following subsections.

\subsubsection{Implementation of the M1 Scheme}

Looking at the general-relativistic Boltzmann equation, i.e., Eq. (2.83), we see that the quantity of interest is the spacetime, angle and frequency dependent specific radiation intensity, $I_{(\nu)}$. Following Thorne (1981), we define the $k$ th moment of this quantity as

$$
M_{(\nu)}^{\alpha_{1} \ldots \alpha_{k}}:=\int_{4 \pi} I_{(\nu)}\left(l^{\alpha_{1}}+u^{\alpha_{1}}\right) \ldots\left(l^{\alpha_{k}}+u^{\alpha_{k}}\right) \mathrm{d} \Omega,
$$

where the four-momentum, $p^{\alpha}=\nu\left(l^{\alpha}+u^{\alpha}\right)$ has been decomposed into a component parallel to the fluid velocity, $u^{\alpha}$, and one orthogonal to it, $l^{\alpha}$. Likewise, the moments of the collisional integral are defined as

$$
S_{(\nu)}^{\alpha_{1} \ldots \alpha_{k}}:=\int_{4 \pi} \mathcal{C}_{(\nu)}^{\mathrm{rad}}\left(l^{\alpha_{1}}+u^{\alpha_{1}}\right) \ldots\left(l^{\alpha_{k}}+u^{\alpha_{k}}\right) \mathrm{d} \Omega .
$$

Taking the covariant derivative of the $k+1$ th moment, i.e., $\nabla_{\beta} M_{(\nu)}^{A_{k} \beta}$, where we follow Thorne's notation $M_{(\nu)}^{A_{k}}:=M_{(\nu)}^{\alpha_{1} \ldots \alpha_{k}}$, yields an evolution equation for this moment. The easiest way to do this is in a local Lorentz frame, where the Christoffel symbols vanish. Applying the chain rule and using the general-relativistic Boltzmann equation then results in (Thorne, 1981; Shibata et al., 2011)

$$
\nabla_{\beta} M_{(\nu)}^{A_{k} \beta}=\frac{\partial}{\partial \nu}\left(\nu M_{(\nu)}^{A_{k} \beta \gamma} \nabla_{\gamma} u_{\beta}\right)+(k-1) M_{(\nu)}^{A_{k} \beta \gamma} \nabla_{\gamma} u_{\beta}+S_{(\nu)}^{A_{k}} .
$$

It is evident that for evolving the $k$ th moment the $k+1$ th moment is required. This results in an infinite series of evolution equations. The information that is lost through the angular integration in Eq. (5.1) is now present in an infinite number of moments $^{1}$. Likewise, the evolution of the infinite hierarchy of Eq. (5.3) is equivalent to the solution of the Boltzmann equation. For creating a practicable numerical scheme, it is necessary to truncate the hierarchy of evolution equations, i.e., only evolve the $k$ lowest moments and use a closure relation for the $k+1$ th moment. The M1 scheme does exactly that for $k=1$.

So far the moments have been introduced as a purely mathematical construct. The lowest moments, however, do have a physical interpretation. Evaluating the

\footnotetext{
${ }^{1}$ Indeed the specific radiation intensity can be recovered from the moments via the infinite series $I_{(\nu)} \propto \sum_{i=0}^{\infty} M_{(\nu)}^{A_{i}}$.
} 
zeroth, first, and second moment in a frame comoving with the fluid, i.e.,

$$
\begin{aligned}
J_{(\nu)} & =\int_{4 \pi} I_{(\nu)} \mathrm{d} \Omega \\
H_{(\nu)}^{\alpha} & =\int_{4 \pi} I_{(\nu)} l^{\alpha} \mathrm{d} \Omega \\
L_{(\nu)}^{\alpha, \beta} & =\int_{4 \pi} I_{(\nu)} l^{\alpha} l^{\beta} \mathrm{d} \Omega
\end{aligned}
$$

we find the radiation field's energy density, momentum density and pressure tensor, respectively. The second moment can be expressed in terms of these fluid-frame quantities as

$$
M_{(\nu)}^{\alpha \beta}=J_{(\nu)} u^{\alpha} u^{\beta}+H_{(\nu)}^{\alpha} u^{\beta}+H_{(\nu)}^{\beta} u^{\alpha}+L_{(\nu)}^{\alpha \beta} .
$$

The total energy-momentum tensor of the radiation field is then simply obtained from the integral over all frequencies, i.e.,

$$
T_{\mathrm{rad}}^{\alpha \beta}=\int_{0}^{\infty} M_{(\nu)}^{\alpha \beta} \mathrm{d} \nu .
$$

The evolution equations for $J_{(\nu)}$ and $H_{(\nu)}$ follow from projections of the secondrank moment evolution equation (5.3) (Shibata et al., 2011). However, since $J_{(\nu)}$ and $H_{(\nu)}$ are evaluated in the fluid-frame, they are not conserved and thus their resulting evolutions equations are not in conservative form (see Sec. 2.2 for the importance of conservative equations).

Evaluating the same quantities in the Eulerian frame, on the other hand, results in a conserved set of variables (in the absence of source terms). Within the $3+1$ formulation (see Sec. 2.1.2) the Eulerian-frame quantities are obtained via temporal, spatial, and mixed spatial-temporal projections. They can be related to the fluidframe quantities via

$$
\begin{aligned}
E_{(\nu)}= & W^{2} J_{(\nu)}+2 W v_{\nu} H_{(\nu)}^{\nu}+v_{\nu} v_{\mu} L_{(\nu)}^{\mu \nu} \\
F_{(\nu)}^{\mu}= & W^{2} v^{\mu} J_{(\nu)}+W\left(g_{\nu}^{\mu}-n^{\mu} v_{\nu}\right) H_{(\nu)}^{\nu}+\left(g_{\nu}^{\mu}-n^{\mu} v_{\nu}\right) v_{\rho} L_{(\nu)}^{\nu \rho}+W v^{\mu} v_{\nu} H_{(\nu)}^{\nu} \\
P_{(\nu)}^{\mu \nu}= & W^{2} v^{\mu} v^{\nu} J_{(\nu)}+W\left(g_{\rho}^{\mu}-n^{\mu} v_{\rho}\right) v^{\nu} H_{(\nu)}^{\rho}+W\left(g_{\rho}^{\nu}-n^{\nu} v_{\rho}\right) v^{\mu} H_{(\nu)}^{\rho} \\
& +\left(g_{\rho}^{\mu}-n^{\mu} v_{\rho}\right)\left(g_{\lambda}^{\nu}-n^{\nu} n_{\lambda}\right) L_{(\nu)}^{\rho \lambda}
\end{aligned}
$$

where all quantities have been defined throughout Chapter 2 and are summarized in Tab. 1.1

The final evolution equations in terms of these Eulerian quantities in the $3+1$ formulation of general relativity then follow as (Shibata et al., 2011; Cardall et al., 2013)

$$
\begin{aligned}
\partial_{t}\left(\sqrt{\gamma} E_{(\nu)}\right) & +\partial_{j}\left(\sqrt{\gamma}\left(\alpha F_{(\nu)}^{j}-\beta^{i} E_{(\nu)}\right)\right)+\frac{\partial}{\partial \nu}\left(\nu \alpha \sqrt{\gamma} M_{(\nu)}^{\alpha \beta \gamma} \nabla_{\gamma} u_{\beta}\right) \\
& =\alpha \sqrt{\gamma}\left(P_{(\nu)}^{i j} K_{i j}-F_{(\nu)}^{j} \partial_{j} \ln \alpha+G_{(0,(\nu))}\right), \\
\partial_{t}\left(\sqrt{\gamma} F_{i(\nu)}\right) & +\partial_{j}\left(\sqrt{\gamma}\left(\alpha P_{i(\nu)}^{j}-\beta^{j} F_{i(\nu)}\right)\right)+\frac{\partial}{\partial \nu}\left(\nu \alpha \sqrt{\gamma} \gamma_{i \alpha} M_{(\nu)}^{\alpha \beta \gamma} \nabla_{\gamma} u_{\beta}\right) \\
& =\sqrt{\gamma}\left(F_{j(\nu)} \partial_{i} \beta^{j}-E_{(\nu)} \partial_{i} \alpha+\frac{\alpha}{2} P_{(\nu)}^{j k} \partial_{i} \gamma_{j k}+\alpha G_{i(\nu)}\right),
\end{aligned}
$$


where we write the collisional source terms on the RHS as

$$
\boldsymbol{G}=\left(\begin{array}{c}
G_{0} \\
G_{i}
\end{array}\right)=\left(\begin{array}{c}
-S_{(\nu)}^{\alpha} n_{\alpha} \\
S_{(\nu)}^{\alpha} \gamma_{i \alpha}
\end{array}\right)
$$

\section{The Gray M1 Scheme}

As pointed out in Sec. 2.3.2 the computational cost of any radiation method can be drastically reduced when only evolving frequency-integrated, i.e., gray, quantities. For this we define the gray moments

$$
J=\int_{0}^{\infty} J_{(\nu)} \mathrm{d} \nu, \quad H^{\alpha}=\int_{0}^{\infty} H_{(\nu)}^{\alpha} \mathrm{d} \nu, \quad L^{\alpha \beta}=\int_{0}^{\infty} L_{(\nu)}^{\alpha \beta} \mathrm{d} \nu .
$$

The same relations, Eqs. (5.9)-(5.11), hold also for the frequency-integrated variables $E, F^{\alpha}$, and $P^{\alpha \beta}$, when dropping the lowerscript $(\nu)$ in the equations. Following the same derivation as above, the frequency-integrated evolution equations are (Shibata et al., 2011)

$$
\begin{aligned}
\partial_{t}(\sqrt{\gamma} E) & +\partial_{j}\left(\sqrt{\gamma}\left(\alpha F^{j}-\beta^{i} E\right)\right) \\
& =\alpha \sqrt{\gamma}\left(P^{i j} K_{i j}-F^{j} \partial_{j} \ln \alpha+G_{0}\right), \\
\partial_{t}\left(\sqrt{\gamma} F_{i}\right) & +\partial_{j}\left(\sqrt{\gamma}\left(\alpha P_{i}^{j}-\beta^{j} F_{i}\right)\right) \\
& =\sqrt{\gamma}\left(F_{j} \partial_{i} \beta^{j}-E \partial_{i} \alpha+\frac{\alpha}{2} P^{j k} \partial_{i} \gamma_{j k}+\alpha G_{i}\right),
\end{aligned}
$$

The collisional source term, $\boldsymbol{G}=\left(-S^{\mu} n_{\mu}, S^{\mu} \gamma_{\mu i}\right)$, depends on the underlying reactions. Including absorption, emission, and iso-energetic scattering it, it takes the form (Bruenn, 1985; Rampp, 2000; Shibata et al., 2011)

$$
S^{\mu}=\eta u^{\mu}-\kappa_{a} J u^{\mu}-\kappa H^{\mu},
$$

where the frequency-integrated emissivity, $\eta$, and the frequency-averaged absorption and total opacity, $\kappa_{a}$ and $\kappa$, respectively, have already been introduced in Sec. 2.3.3. The computation of this source term requires a transformation of the variables in the Eulerian frame to the fluid frame. From the energy-momentum tensor

$$
T_{\mathrm{rad}}^{\mu \nu}=E n^{\mu} n^{\nu}+F^{\mu} n^{\nu}+F^{\nu} n^{\mu}+P^{\mu \nu}
$$

follows

$$
\begin{aligned}
J & =W^{2}\left(E-2 F^{\mu} v_{\mu}+P^{\mu \nu} v_{\mu} v_{\nu}\right) \\
H^{\mu} & =W\left(e-F^{\nu} v_{\nu}\right) h_{\rho}^{\mu} n^{\rho}+W h_{\nu}^{\mu} F^{\nu}-W h_{\nu}^{\mu} v_{\rho} P^{\nu \rho} .
\end{aligned}
$$

\section{The Closure Relation}

As mentioned before and also evident from Eqs. (5.16) and (5.17) the pressure tensor $P^{i j 2}$ is required in order to close the system of equations. The exact form of this tensor differs from problem to problem (Thorne, 1981; Rezzolla \& Miller, 1994) and

\footnotetext{
${ }^{2}$ Note that $P^{0 \nu}=P^{\mu 0}=0$, i.e., $P^{\mu \nu}$ is a purely spatial tensor. The same is true for $F^{\mu}$.
} 
is generally not known. It is, however, possible to write $P^{i j}$ as a function of the evolved variables, $E$ and $F^{i}$, in the optically infinitely thin (free streaming) and thick (diffusion) limit. The general closure then follows as a simple interpolation between these limits, i.e.,

$$
P^{i j}=\frac{3 \chi(\xi)-1}{2} P_{\text {thin }}^{i j}+\frac{3(1-\chi(\xi))}{2} P_{\text {thick }}^{i j},
$$

where $\chi(\xi)$ is the closure function. This closure function depends on the variable Eddington factor defined as

$$
\xi:=\sqrt{\frac{h_{\mu \nu} H^{\mu} H^{\nu}}{J^{2}}}=\sqrt{\frac{H^{\mu} H_{\mu}}{J^{2}}}
$$

where the second equality holds due to $H^{\mu} u_{\mu} \equiv 0$. In general relativity one can also choose

$$
\xi:=\sqrt{\frac{F^{\mu} F_{\mu}}{E^{2}}}
$$

instead. Both choices for $\xi$ lead to $\xi=1$ in the free-streaming limit. In the diffusion limit, on the other hand, $\xi=0$ (Levermore, 1984; Shibata et al., 2011), which can only be fulfilled with the former choice. Choosing Eq. (5.23) also means a substantially increased numerical cost for the method due to the necessity of a root-finding method, which will be detailed below.

Within the implementation of FRAC we choose the Minerbo closure (Minerbo, 1978), which is also referred to as maximum-entropy closure and reads

$$
\chi(\xi)=\frac{1}{3}+\xi^{2} \frac{6-2 \xi+6 \xi^{2}}{15} .
$$

Other choices for the closure can be found in the literature, one of the most common ones being the Levermore closure (Levermore, 1984). An extensive comparison of different closures can be found in Murchikova et al. (2017).

The missing ingredient for computing the pressure according to Eq. (5.22) is the pressure tensor in the thin and thick limit, i.e., $P_{\text {thin }}^{i j}$ and $P_{\text {thick }}^{i j}$, respectively. In the former case we use

$$
P_{\mathrm{thin}}^{i j}=\frac{F^{i} F^{j}}{F^{k} F_{k}} E
$$

which has been shown to violate causality when not in the free-streaming limit, but still represents the best choice considering the limitations of proposed alternatives (Shibata et al., 2011). In the optically thick limit the radiation variables are well known in the fluid frame, i.e.,

$$
\begin{aligned}
J_{\text {thick }} & =\frac{3}{2 W^{2}+1}\left[\left(2 W^{2}-1\right) E-2 W^{2} F^{i} v_{i}\right], \\
\left(H_{i}\right)_{\text {thick }} & =\frac{F_{i}}{W}+\frac{W v_{i}}{2 W^{2}+1}\left[\left(4 W^{2}+1\right) F^{j} v_{j}-4 W^{2} E\right], \\
L_{\text {thick }}^{\mu \nu} & =\frac{J_{\text {thick }}}{3} h^{\mu \nu} .
\end{aligned}
$$

The pressure, $P_{\text {thick }}^{i j}$ can then simply be computed using Eq. (5.11).

The closure for computing $P^{i j}$ needs the variable Eddington factor, $\xi$, which depends on $H^{\mu}$ and $J$, which can be computed from Eqs. (5.20) and (5.21), which in turn require $P^{i j}$. It is thus evident that a root-finding method is required for obtaining $P^{i j}$. In FRAC we follow Foucart et al. (2015) and use the following method: 
1.) Compute $P_{\text {thin }}^{i j}$ and $P_{\text {thick }}^{i j}$ from $E$ and $F_{i}$.

2.) Compute $P^{i j}$ according to Eq. (5.22) using $\xi$ from the previous timestep. Use this $P^{i j}$ for computing $J$ and $H^{\mu}$ according to Eqs. (5.21) and (5.21).

3.) If the function

$$
f(\xi)=\frac{J^{2} \xi^{2}-H^{\mu} H_{\mu}}{E^{2}}
$$

is below a specified threshold value, we have found the correct $\xi$ and hence the correct pressure tensor. If not, we adjust $\xi$ using a Newton-Raphson method, i.e.,

$$
\xi_{\text {new }}=\xi-\frac{f(\xi)}{f^{\prime}(\xi)}
$$

and cycle back to step 2 .

Alternatively, one could use Brent's method for finding the root of $f(\xi)$, which is more robust, but also computationally more expensive.

This variable Eddington factor method - albeit computationally more expensive is the most accurate method for closing the system of equations within the M1 method. It allows an exact evolution in the optically thin and thick limit ${ }^{3}$ and gives a reasonable estimate for the intermediate regime (see also Foucart et al., 2018; Sumiyoshi et al., 2020, for the error of this closure method). Other closures, like the commonly used (see e.g., Roedig et al., 2012; Fragile et al., 2012) closure given by

$$
P^{i j}=P_{\text {thick }}^{i j},
$$

are only accurate in a certain regime, like the optically thick one, where $F^{i} F_{i} \ll E^{2}$.

\section{Computation of the Fluxes}

Within the M1 scheme, the radiation is treated like a fluid (often referred to as radiation fluid), which is also evident from the evolution equations. Such a fluid can exhibit shocks, which can not be captured when using a standard finite-difference approach for computing the derivative for the flux-term. We therefore use the reconstruct-solveupdate method introduced in Sec. 2.2. Specifically, we implement the minmod slope limiter, i.e., Eq. (2.72) for reconstructing the left and right states at the interfaces according to Eqs. (2.70) and (2.71) and the HLLE Riemann solver, i.e., Eq. (2.74). Note that in order to ensure causality we reconstruct the variables $\left(E, F_{i} / E\right)$ rather than $\left(E, F_{i}\right)$.

The left- and right-going characteristic speeds, $\lambda_{L}$ and $\lambda_{R}$, are obtained as the Eigenvalues of the Jacobian matrix of the flux terms (Font et al., 1994; Banyuls et al., 1997). The solution of this eigenvalue problem depends on the pressure tensor. In the optically thin regime we find for the characteristic speeds in $i$ th direction

$$
\lambda_{R, \text { thin }}=-\beta^{i}+\alpha \frac{F^{i}}{\sqrt{F_{j} F^{j}}}, \quad \lambda_{L, \text { thin }}=-\beta^{i}-\alpha \frac{F^{i}}{\sqrt{F_{j} F^{j}}},
$$

and for the thick limit

$$
\lambda_{R, \text { thick }}=\max \left(-\beta+p^{i}, \Lambda_{+}\right), \quad \lambda_{L, \text { thick }}=\min \left(-\beta+p^{i}, \Lambda_{-}\right),
$$

\footnotetext{
${ }^{3}$ Note that for correctly reproducing the diffusion limit also the fluxes in Eqs. (5.16) and (5.17) have to be corrected, which will be discussed below.
} 
with $p^{i}:=\alpha v^{i} / W$ and

$$
\Lambda_{ \pm}=-\beta^{i}+\frac{2 W^{2} p^{i} \pm \sqrt{\alpha^{2} \gamma^{i i}\left(2 W^{2}+1\right)-2\left(W p^{i}\right)^{2}}}{2 W^{2}+1} .
$$

In order to obtain $\lambda_{R / L}$ in all regimes, we use the same closure relation as for the pressure tensor, i.e.,

$$
\lambda_{R / L}=\frac{3 \chi(\xi)-1}{2} \lambda_{R / L, \text { thin }}+\frac{3(1-\chi(\xi))}{2} \lambda_{R / L, \text { thick }} .
$$

For Eq. (5.36) we can reuse $\xi$, which has already been computed on cell-interfaces after the reconstruction step.

Using $\lambda_{R / L}$ in the HLLE-Riemann solver, yields the fluxes $\mathcal{F}_{E}^{i}$ and $\mathcal{F}_{F_{j}}^{i}$ in $i$ th direction. In the optically thick limit, these fluxes are inaccurate and need to be corrected in order to obtain the correct asymptotic behavior of the system. More specifically, Jin \& Levermore (1996) have shown that for stiff source terms, in this case $G_{0}$ and $G_{i}$ as will also be discussed below, lead to an inaccurate solution of the system on a timescale of $\mathcal{O}(1 / \kappa \Delta x)$. In the case of radiation this results in an incorrect diffusion rate, i.e., an inaccurate rate at which the radiation is scattered by the fluid. Following O'Connor (2015); Foucart et al. (2015), we thus apply a correction to the fluxes of the form

$$
\mathcal{F}_{E, \mathrm{corr}}^{i}=a \mathcal{F}_{E}^{i}+(1-a) \mathcal{F}_{E, \text { asym }}^{i}
$$

The weight function is

$$
a=\tanh \left(\frac{1}{\kappa_{\ell+1 / 2} \Delta x^{i}}\right)
$$

where we approximate $\kappa$ at the cell interface between the $\ell$ th and $\ell+1$ th cell as $\kappa=\sqrt{\kappa_{\ell} \kappa_{\ell+1}}$ and use the proper distance in direction $i$ between two cells, i.e., $\Delta x^{i}=$ $\sqrt{\left.\gamma_{i i}\left(\Delta x_{\text {grid }}^{i}\right)^{2}\right)}$, where $\Delta x_{\text {grid }}^{i}$ is the coordinate distance. The asymptotic flux is known in the fluid frame and can be used together with Eq. (5.10) in order to find the correct asymptotic flux in the Eulerian frame as

$$
\mathcal{F}_{E, \text { asym }}^{i}=\sqrt{\gamma}\left(\frac{4}{3} W^{2} \alpha v^{i} J_{\text {thick }}-\beta^{i} E\right)-\frac{\alpha W}{3 \kappa_{\ell+1 / 2}} \sqrt{\gamma}\left(\gamma^{i j}+v^{i} v^{j}\right) \frac{\mathrm{d} J_{\text {thick }}}{\mathrm{d} x^{j}} .
$$

The main difficulty in obtaining this flux lies in the computation of the total derivative of $J_{\text {thick }}$ on the cell-interfaces. As a simple approximation we simply compute

$$
\frac{\mathrm{d} J_{\text {thick }}}{\mathrm{d} x^{i}}=\frac{J_{\text {thick }, \ell+1}-J_{\text {thick }, \ell}}{\Delta x_{\text {grid }}^{i}} .
$$

For the first term in Eq. (5.39) we compute the advection speed in $i$ th direction, i.e.,

$$
c_{\mathrm{adv}}=-\beta^{i}+4 \alpha \frac{W^{2}}{2 W^{2}+1} v^{i} .
$$

If this advection speed is positive for both left and right states, we choose to compute the first term in Eq. (5.39) from the left reconstructed state. If $c_{\text {adv }}$ is negative, we compute this term from the right reconstructed state. In all other cases we simply set this term to zero. 
Finally, we also correct $\mathcal{F}_{F_{i}}^{i}$. Following Audit et al. (2002) we use

$$
\mathcal{F}_{F_{i}, \text { corr }}^{j}=a \mathcal{F}_{F_{i}}^{j}+(1-a)\left(\frac{\mathcal{F}_{F_{i}, \ell+1}^{j}-\mathcal{F}_{F_{i}, \ell}^{j}}{2}\right) \text {. }
$$

Note that in Foucart et al. (2015) the weight for correcting $\mathcal{F}_{F_{i}}^{j}$ is $b=1 /\left(\kappa_{i+1 / 2} \Delta x^{j}\right)$. This weight diverges for $\kappa \rightarrow 0$, which is why we rather choose $a$ as weight function.

\section{Implicit Treatment of Stiff Source Terms}

In general, the opacities $\kappa_{a}$ and $\kappa_{s}$ as well as the emissivity $\eta$ change over several orders of magnitude. From Eq. (5.18) it is clear that this causes the entire collisional source term, $\boldsymbol{G}$, to also have such variations. Whenever the numerical value (in code units) becomes much larger than the numerical value of the evolved variables, the evolution becomes numerically unstable when using an explicit time stepper. This can be mitigated by decreasing the time step $\Delta t$. Specifically, the time step needs to be of order $\mathcal{O}(1 / \kappa)$ and $\mathcal{O}(1 / \eta)$ in order to ensure stability. Such a small timestep would make any realistic simulation unfeasible. It is therefore desirable to use a time stepper that allows for a stable evolution with a constant time step independent of how large the source term is.

An implicit solver provides exactly this feature by treating the corresponding source term implicitly rather than explicitly. Such a solver, however, comes with the drawback that at every iteration an implicit equation needs to be solved, which is in general not trivially possible and requires costly root-finding methods. A partial solution is provided by an implicit-explicit (IMEX) scheme (Pareschi \& Russo, 2005), which only treats the stiff part of the sources implicitly and the rest explicitly. Such an IMEX method is frequently used in resistive MHD, where the resistivity causes the system of equations to become stiff (seee.g., Palenzuela et al., 2009; Dionysopoulou et al., 2013; Alic et al., 2012b; Ripperda et al., 2019).

In general, within such an IMEX scheme the state of the evolved variables at the $n+1$ th iteration is obtained from the one after $n$ iteration via $N$ substeps. Numerically, this time stepper is written as (Pareschi \& Russo, 2005)

$$
\begin{gathered}
\boldsymbol{U}^{(i)}=\boldsymbol{U}^{n}+\Delta t \sum_{j<i} \tilde{a}_{i j} \boldsymbol{X}\left(\boldsymbol{U}^{(j)}\right)+\Delta t \sum_{j \leq i} a_{i j} \boldsymbol{M}\left(\boldsymbol{U}^{(j)}\right), \\
\boldsymbol{U}^{n+1}=\boldsymbol{U}^{n}+\Delta t \sum_{i=0}^{N} \tilde{w}_{i} \boldsymbol{X}\left(\boldsymbol{U}^{(i)}\right)+\Delta t \sum_{i=0}^{N} w_{i} \boldsymbol{M}\left(\boldsymbol{U}^{(i)}\right),
\end{gathered}
$$

where the intermediate state of evolved variables $\boldsymbol{U}^{(i)}$ is computed from a combination of the explicit terms, $\boldsymbol{X}$, and the implicit terms, $\boldsymbol{M}$. The coefficient matrices $\left[\tilde{a}_{i j}\right]$ and $\left[a_{i j}\right]$ as well as the weights $\tilde{w}_{i}$ and $w_{i}$ can be expressed in form of a Butcher tableau and determine the type of the IMEX scheme. Here we use a diagonally implicit Runge-Kutta (DIRK) scheme, for which $\tilde{a}_{i j}=0$ for $j \geq i$ and $a_{i j}=0$ for $j>i$. Specifically, we use the second-order SS2 $(2,2,2)$ scheme (Pareschi \& Russo, 2005), which is written as 


$$
\begin{aligned}
\boldsymbol{U}^{(0)} & =\boldsymbol{U}^{n}+\Delta t \gamma \boldsymbol{M}\left(\boldsymbol{U}^{(0)}\right) \\
\boldsymbol{U}^{(1)} & =\boldsymbol{U}^{n}+\Delta t \gamma \boldsymbol{X}\left(\boldsymbol{U}^{(0)}\right)+\Delta t\left((1-2 \gamma) \boldsymbol{M}\left(\boldsymbol{U}^{(0)}\right)+\gamma \boldsymbol{M}\left(U^{(1)}\right)\right) \\
\boldsymbol{U}^{n+1} & =\boldsymbol{U}^{n}+\frac{1}{2} \Delta t\left(\boldsymbol{X}\left(\boldsymbol{U}^{(0)}\right)+\boldsymbol{X}\left(\boldsymbol{U}^{(1)}\right)\right)+\frac{1}{2} \Delta t\left(\boldsymbol{M}\left(\boldsymbol{U}^{(0)}\right)+\boldsymbol{M}\left(\boldsymbol{U}^{(1)}\right)\right)
\end{aligned}
$$

where $\gamma=1-1 / \sqrt{2}$. When implementing this scheme, we avoid storing the explicit source, $\boldsymbol{X}$, by rewriting the above set of equations as

$$
\begin{aligned}
\boldsymbol{U}^{(0)} & =\left\{\boldsymbol{U}^{n}\right\}+\Delta t \gamma \boldsymbol{M}^{(0)} \\
\boldsymbol{U}^{(1)} & =\left\{\frac{3 \gamma-1}{\gamma} \boldsymbol{U}^{n}+\frac{1-2 \gamma}{\gamma} \boldsymbol{U}^{(0)}+\Delta t \boldsymbol{X}^{(0)}\right\}+\Delta t \gamma \boldsymbol{M}^{(1)} \\
\boldsymbol{U}^{n+1} & =\left\{\frac{1}{2}\left(\boldsymbol{U}^{n}+\boldsymbol{U}^{(1)}+\Delta t \boldsymbol{X}^{(1)}\right)+\Delta t\left(\gamma \boldsymbol{M}^{(0)} \frac{1-\gamma}{2} \boldsymbol{M}^{(1)}\right)\right\},
\end{aligned}
$$

where we introduce $\boldsymbol{M}^{(i)} / \boldsymbol{X}^{(i)}:=\boldsymbol{M}\left(\boldsymbol{U}^{(i)}\right) / \boldsymbol{X}\left(\boldsymbol{U}^{(i)}\right)$ as more convenient notation. Furthermore, the explicit terms in Eqs. (5.48)-(5.50) are surrounded by curly braces. This notation helps to recognize that at each substep an equation of the form

$$
\boldsymbol{U}^{(i)}=\boldsymbol{U}^{\prime}+\text { const. } \cdot \boldsymbol{M}^{(i)}
$$

has to be solved with $\boldsymbol{U}^{\prime}$ being an intermediate state that includes all explicit contributions, i.e., all terms in curly braces.

For the M1 system, we can identify the explicit contributions as

$$
\boldsymbol{X}^{(i)}=\left(\begin{array}{c}
-\partial_{k} \mathcal{F}_{E, \mathrm{corr}}^{k}+\sqrt{\gamma} \alpha\left(P^{k l} K_{k l}-F^{k} \partial_{k} \ln \alpha\right) \\
-\partial_{k} \mathcal{F}_{F_{j}, \mathrm{corr}}^{k}+\sqrt{\gamma}\left(F_{k} \partial_{j} \beta^{k}-E \partial_{j} \alpha+\frac{\alpha}{2} P^{k l} \partial_{j} \gamma_{k l}\right)
\end{array}\right)
$$

which corresponds to the flux terms and the geometric source terms. The implicit sources, on the other hand, can be identified with the collisional source term

$$
\boldsymbol{M}^{(i)}=\sqrt{\gamma} \alpha\left(\begin{array}{c}
-S^{\mu} n_{\mu} \\
S^{\mu} \gamma_{\mu j}
\end{array}\right)=\sqrt{\gamma} \alpha\left(\begin{array}{c}
G_{0} \\
G_{i}
\end{array}\right)
$$

Unfortunately, in this case the implicit equation (5.51) can not be solved analytically. In order to refrain from computationally expensive root-finding methods, we linearize the implicit source term, i.e., we write

$$
\boldsymbol{M}^{(i)}=\widehat{\mathcal{M}} \boldsymbol{U}^{(i)}+\boldsymbol{b} .
$$

This linearization is only possible when assuming that the fluid velocity and the radiation pressure tensor only change marginally during each timestep, hence it is only approximate. The matrix $\widehat{\mathcal{M}}$ and the column vector $\boldsymbol{b}$ can be obtained when replacing the fluid frame variables in the collisional source term with the ones in the Eulerian frame. Using Eqs. (5.9) and (5.10) we find

$$
\begin{aligned}
S^{\mu}= & \left(\kappa_{s} W^{2} u^{\mu}-\kappa W n^{\mu}\right) E+\left(\kappa W v^{i} n^{\mu}-2 \kappa_{s} W^{2} u^{\mu} v^{i}\right) F_{i} \\
& +\left(\eta u^{\mu}-\kappa_{a} W^{2} u^{\mu} v_{i} v_{j} P^{i j}+\kappa W h_{i}^{\mu} v_{j} P^{i j}\right)+\kappa W F^{\mu} .
\end{aligned}
$$

This term can not yet be written in the desired linear form, i.e., as $A^{\mu} E+B^{\mu i} F_{i}+C^{\mu}$. This will only be possible after contracting $S^{\mu}$ with $n_{\mu}$ and $\gamma_{\mu j}$, as is required for $G_{0}$ 
and $G_{j}$, respectively. These contractions then yield the $4 \times 4$ matrix

$$
\begin{aligned}
& \widehat{\mathcal{M}}=\sqrt{\gamma} \alpha \times \\
& \left(\begin{array}{cc}
\kappa_{s} W^{3}-\kappa W & \left(\kappa-2 W^{2} \kappa_{s}\right) W v_{x} \\
\left(\kappa_{s} W^{2} u^{j}-\kappa W n^{j}\right) \gamma_{j x} & \left(\kappa n^{j}-2 \kappa_{s} W u^{j}\right) \gamma_{j x} W v^{x}-\kappa W \\
\left(\kappa_{s} W^{2} u^{j}-\kappa W n^{j}\right) \gamma_{j y} & \left(\kappa n^{j}-2 \kappa_{s} W u^{j}\right) \gamma_{j y} W v^{x} \\
\left(\kappa_{s} W^{2} u^{j}-\kappa W n^{j}\right) \gamma_{j z} & \left(\kappa n^{j}-2 \kappa_{s} W u^{j}\right) \gamma_{j z} W v^{x} \\
& \\
& \\
\left(\kappa-2 W^{2} \kappa_{s}\right) W v_{y} & \left(\kappa-2 W^{2} \kappa_{s}\right) W v_{z} \\
\left(\kappa n^{j}-2 \kappa_{s} W u^{j}\right) \gamma_{j x} W v^{y} & \left(\kappa n^{j}-2 \kappa_{s} W u^{j}\right) \gamma_{j x} W v^{z} \\
\left(\kappa n^{j}-2 \kappa_{s} W u^{j}\right) \gamma_{j y} W v^{y}-\kappa W & \left(\kappa n^{j}-2 \kappa_{s} W u^{j}\right) \gamma_{j y} W v^{z} \\
\left(\kappa n^{j}-2 \kappa_{s} W u^{j}\right) \gamma_{j z} W v^{y} & \left(\kappa n^{j}-2 \kappa_{s} W u^{j}\right) \gamma_{j z} W v^{z}-\kappa W
\end{array}\right)
\end{aligned}
$$

and the column vector

$$
\boldsymbol{b}=\sqrt{\gamma} \alpha\left(\begin{array}{c}
\eta W+\kappa_{s} W P^{\mu \nu} u_{\mu} u_{\nu} \\
\left(P^{\mu \nu} u_{\mu}\left(\kappa h_{\nu}^{\alpha}-\kappa_{a} u_{\nu} u^{\alpha}\right)+\eta u^{\alpha}\right) \gamma_{\alpha x} \\
\left(P^{\mu \nu} u_{\mu}\left(\kappa h_{\nu}^{\alpha}-\kappa_{a} u_{\nu} u^{\alpha}\right)+\eta u^{\alpha}\right) \gamma_{\alpha y} \\
\left(P^{\mu \nu} u_{\mu}\left(\kappa h_{\nu}^{\alpha}-\kappa_{a} u_{\nu} u^{\alpha}\right)+\eta u^{\alpha}\right) \gamma_{\alpha z}
\end{array}\right) .
$$

Using Eqs. (5.20) and (5.21) it can easily be verified that

$$
\widehat{\mathcal{M}}\left(\begin{array}{c}
E \\
F_{x} \\
F_{y} \\
F_{z}
\end{array}\right)+\boldsymbol{b}=\sqrt{\gamma} \alpha\left(\begin{array}{c}
-S^{\mu} n_{\mu} \\
S^{\mu} \gamma_{\mu x} \\
S^{\mu} \gamma_{\mu y} \\
S^{\mu} \gamma_{\mu z}
\end{array}\right)=\left(\begin{array}{c}
G_{0} \\
G_{x} \\
G_{y} \\
G_{z}
\end{array}\right)
$$

with $S^{\mu}$ given by Eq. (5.18).

Using this linearization of the implicit source term now allows for solving Eq. (5.51) at every substep as follows:

1.) Compute $\boldsymbol{U}^{\prime}$ from $\boldsymbol{U}^{n}, \boldsymbol{U}^{(j)}$ and $\boldsymbol{X}^{(j)}$, where $j<i$ with $i$ the number of the current substep.

2.) Compute the matrix $\widehat{\mathcal{M}}$ and the column vector $\boldsymbol{b}$ from the variables of the previous substep. Inserting Eq. (5.54) into Eq. (5.51) yields a linear equation, whose solution is

$$
\boldsymbol{U}^{(i)}=\left(\widehat{\mathcal{I}}-a_{i i} \Delta t \widehat{\mathcal{M}}\right)^{-1}\left(\boldsymbol{U}^{\prime}+a_{i i} \Delta t \boldsymbol{b}\right)
$$

with $\widehat{\mathcal{I}}$ being the unit matrix.

3.) Use the updated $\boldsymbol{U}^{(i)}$ for computing $\boldsymbol{M}^{(i)}$ according to Eq. (5.54). This $\boldsymbol{M}^{(i)}$ needs to be stored and will be required for the final substep for computing $U^{n+1}$.

Note that this procedure is general and thus works for any IMEX method of arbitrary order. This linearization is the same as used by Foucart et al. (2015), although not within an IMEX scheme.

A more accurate method, which can easily be implemented as direct extension of the above procedure, is the so-called fixed-point method. In the context of a radiation field back-reacting onto a fluid, the implicit source $\boldsymbol{M}^{(i)}$ can be used to also update 
the fluid variables (see also below for details on the coupling between radiation and fluid). The updated fluid three-velocity can then be used together with the updated radiation field to recompute the radiation pressure tensor. One then cycles back to step 2 using the new velocity and pressure for getting a more precise estimate for $\widehat{\mathcal{M}}$ and $\boldsymbol{b}$. This cycle is repeated until a desired degree of convergence is achieved. This method is implemented by Roedig et al. (2012); Melon Fuksman \& Mignone (2019) in the context of radiation and by Palenzuela et al. (2009); Dionysopoulou et al. (2013); Alic et al. (2012b); Ripperda et al. (2019) in the context of resistive MHD.

Another alternative would be a direct root-finding as used in McKinney et al. (2014); Sa̧dowski et al. (2013); Melon Fuksman \& Mignone (2019); Ripperda et al. (2019). In the case of radiation with four evolved variables, one could use, for example, a four-dimensional Newton-Raphson method. Of course, this comes with an increased computational cost, which is why we here stick to the approximate method making use of the linearization.

\section{Coupling with the Fluid}

The dependence of the radiation on the fluid variables is encoded in the emissivity and the opacities. The back-reaction of the radiation onto the fluid is modeled via an additional source term that is added to the conservative fluid equations [see Eqs. (2.61), (2.65), and (2.66)]. Specifically, we modify the energy and momentum equations by adding a source as in Eq. (2.95).

In the following we keep the previous notation for the radiation variables and mark the corresponding fluid variables explicitly with a subscript "fl" for an easy distinction. We can then write this additional fluid source term as

$$
\boldsymbol{M}_{\mathrm{fl}}=-\boldsymbol{M}=-\sqrt{\gamma} \alpha\left(\begin{array}{c}
G_{0} \\
G_{i}
\end{array}\right)=-\widehat{\mathcal{M}}\left(\begin{array}{c}
E \\
F_{i}
\end{array}\right)-\boldsymbol{b} .
$$

This source term can be treated explicitly only when the fluid dominates, i.e., when the fluid variables are numerically much larger than this source term. Since this is generally not the case, we have to also treat this term implicitly. However, since $\boldsymbol{M}$ is already known after solving the implicit equations for the radiation, $\boldsymbol{M}_{\mathrm{fl}}$ is also known and the solution of the implicit equation for the fluid is thus trivial. This is of course only the case as long as the fluid velocity is assumed to be constant during each substep. Otherwise, one would have to employ an eight-dimensional root-finding (four radiation and four fluid variables) or make use of the fixed-point method as outlined above.

Within our approximate method it is possible to recompute $P^{\mu \nu}$ after obtaining $U^{(i)}$ (i.e., the state of the radiation variables), so that a more accurate value for $\boldsymbol{M}_{\text {fluid }}^{(i)}$ could be computed. However, this would break energy-momentum conservation, because then $\boldsymbol{M}_{\mathrm{fl}} \neq \boldsymbol{M}$.

If the fluid's composition is also evolved (as is typically the case for binary neutron star simulations), the system of conservative fluid evolution equations is extended by an advection equation for the electron fraction, $Y_{e}$. In the presence of radiation in the form of neutrinos, $Y_{e}$ is no longer only advected. Instead, the neutrinos will change the composition dynamically through charged-current reactions. This is modeled via the addition of a source term to the advection equation (Foucart et al., 2015), i.e.,

$$
\partial_{t} Y_{e}=\cdots-\sum_{\nu_{i}} \operatorname{sign}\left(\nu_{i}\right) \alpha \sqrt{\gamma} \frac{\eta-\kappa_{a} J}{\bar{\epsilon}_{\nu_{i}}}
$$




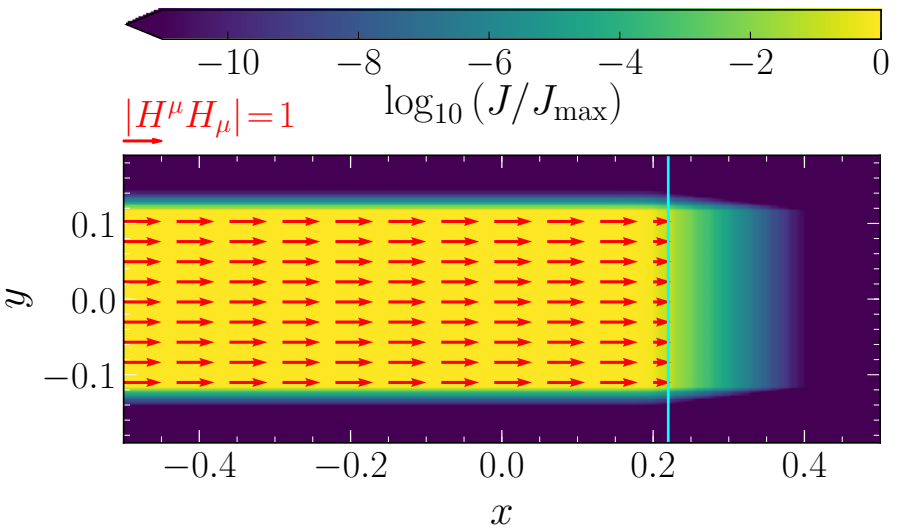

Figure 5.1: Propagating beam of radiation that is injected into the grid from the left boundary parallel to the $\mathrm{x}$-axis. While the color-code shows the radiation energy density, the momentum density is shown by red arrows. The cyan line indicates the distance $x=t$, where $t$ is the elapsed simulation time (in units of $c=1$ ). Reprint of Fig. 1 in Weih et al. (2020c).

where $\bar{\epsilon}_{\nu_{i}}$ is the mean energy of neutrinos of type $\nu_{i}$ and the sign-function evaluates to 1 for electron neutrinos, -1 for anti-electron neutrinos, and 0 for all other type of neutrinos. Again, this source term can be computed from $E$ and $F_{i}$ after solving the implicit equation for the radiation variables ${ }^{4}$. For the remainder of this Chapter we focus on fluids composed of only one type of particles, thus neglecting Eq. (5.61).

Within FRAC, which is a stand-alone code for evolving only the radiation, we solve the implicit equation (5.51) for the radiation by computing $\widehat{\mathcal{M}}$ and $\boldsymbol{b}$ for a given input of fluid and metric variables. The code then has the option to return $\boldsymbol{M}_{\mathrm{ff}}$ to a code that evolves the fluid variables. This works for both, stationary as well as dynamically evolving spacetimes, because FRAC can receive the state of the metric at every iteration.

For the simulations carried out in the remainder of Sec. 5.1 we couple FRAC to the Black Hole Accretion Code (BHAC) developed by Porth et al. (2017). BHAC solves the conservative GRMHD equations in an arbitrary, but stationary spacetime. It is based on the open-source MPI-AMRVAC framework (Keppens et al., 2012; Porth et al., 2014) and handles Cartesian as well as spherical coordinates in one, two and three dimension making use of adaptive block based oct-tree mesh refinement. For the purpose of coupling BHAC with FRAC we implement the SS2 $(2,2,2)$ IMEX method in BHAC and extend the internal arrays to hold four more variables, i.e., the radiation variables $E$ and $F_{i}$. In this way, BHAC passes the entire array of evolved variables (fluid, radiation, and metric) to FRAC at every substep and receives the updated radiation variables as well as the implicit source $\boldsymbol{M}_{\mathrm{fl}}$. The latter is than used to trivially update also the fluid variables. Note that FRAC itself does not know the underlying grid structure (it only receives the grid spacing $\Delta x_{\text {grid }}^{i}$ for computing derivatives) or the underlying coordinates, which is not necessary since Eqs. (5.16) and (5.17) are written in a covariant form. The I/O, distributed-memory parallelisation and gridstructure is completely handled by BHAC. For making this possible, FRAC also returns the radiation fluxes $\mathcal{F}_{E \text {,corr }}^{j}$ and $\mathcal{F}_{F_{i} \text {,corr }}^{j}$ to BHAC for computing the correct fluxes at interfaces between coarse and fine grids.

\footnotetext{
${ }^{4}$ In practice, the evaluation of $\bar{\epsilon}_{\nu_{i}}$ requires some effort, because in a gray scheme no information about the radiation's energy dependence is present. Possible methods for finding a good estimate within a gray scheme are discussed in Foucart et al. (2015, 2016a).
} 


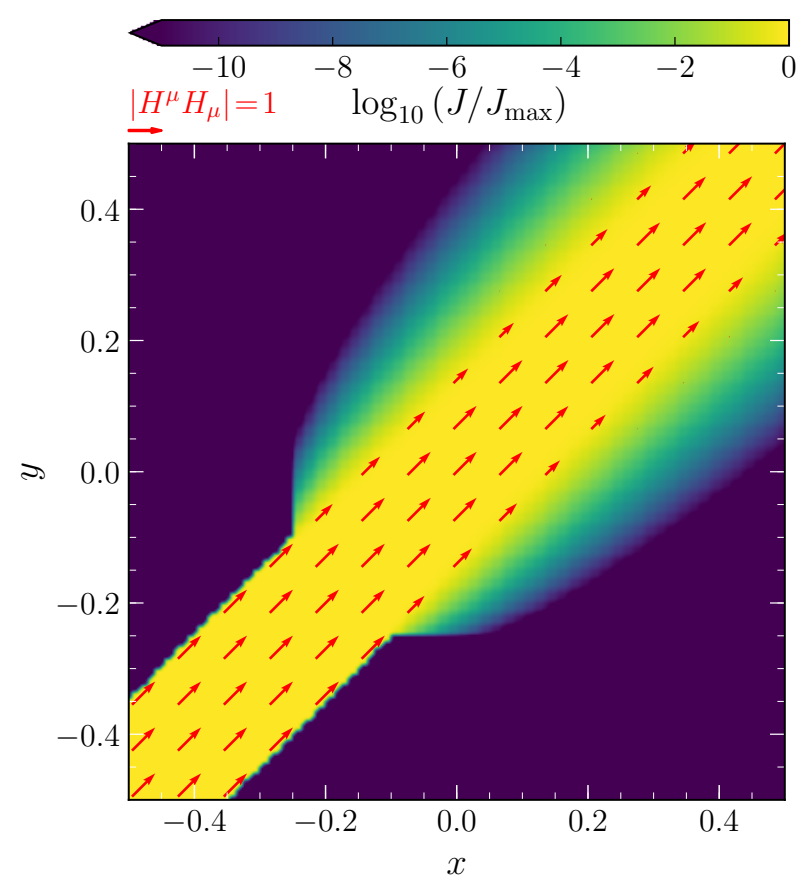

Figure 5.2: Same as Fig. 5.1, but for a beam traveling diagonally. The region defined by $x, y<-0.25$ is frozen ensuring a constant inflow of radiation. Reprint of Fig. 2 in Weih et al. (2020c).

\subsubsection{Code Tests}

We start by verifying the implementation of FRAC with a number of standard tests, first in special-relativity and later also for the general-relativistic case.

\section{Beam Tests}

The first and probably simplest test is that of a straight beam of radiation. The expectation is that a straight beam will remain collimated and propagate at the speed of light. For testing this, we fix the metric to be the Minkowski one and set all fluid variables to zero. As a consequence there is no more distinction between fluid and Eulerian frame, i.e., $E \equiv J$ and $F^{\mu} \equiv H^{\mu}$. On a two-dimensional Cartesian grid of size $-0.5<x<0.5,-0.2<y<0.2$ covered by $100 \times 40$ grid cells we initialize the radiation as

$$
J=\left\{\begin{array}{ll}
1, & x<-0.4 \text { and }|y|<0.12 \\
10^{-15}, & \text { otherwise }
\end{array}\right. \text {. }
$$

The momentum density is initialized as $H_{x}=J, H_{y}=H_{z}=10^{-15}$. This setup corresponds to a beam that is shot into the grid from the left propagating at the speed of light parallel to the x-axis. A boundary condition that simply copies the final layer of the physical grid ensures that the beam is continuously injected. Opacities and emissivity are set to zero allowing the beam to propagate in vacuum.

The result of this test is shown in Fig. 5.1, where the vertical cyan lines shows the distance the beam should have traveled if it were to propagate at the speed of light. The logarithmic presentation of the radiation energy density reveals a small amount of diffusion that propagates ahead of the beam, which is a standard behavior for finite-difference codes. 


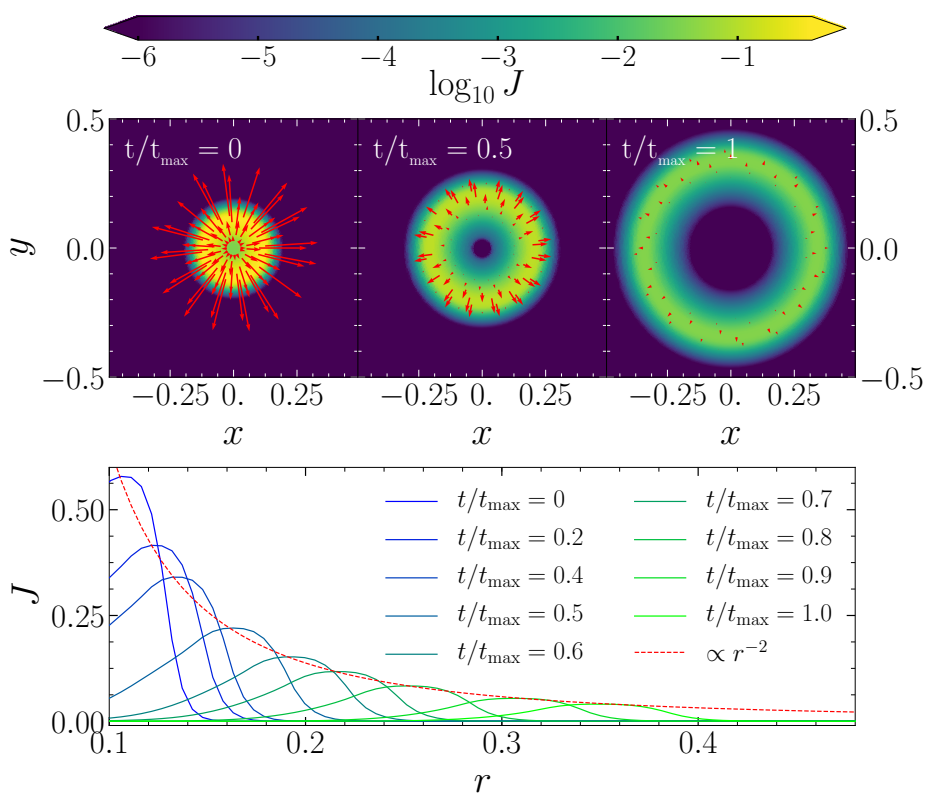

FiguRE 5.3: Top: Same as Fig. 5.1, but for a freely propagating radiation wave, at three representative times. Bottom: Colored lines show diagonal profiles of $J$ at nine different times. The red-dashed lines is a function proportional to $1 / r^{2}$ fitted to the maxima of these profiles. Reprint of Fig. 4 in Weih et al. (2020c).

A more demanding test is that of a beam traveling diagonally, i.e., not parallel to one of the grid's axes. Fig. 5.2 shows that the diffusion is much larger in this case, but the flux is still confined to the central region of the beam.

The next step would be to test the behavior of two crossing beams. It is well known that the M1 scheme can not reproduce the correct behavior in this case (Fragile et al., 2014; McKinney et al., 2014; Foucart et al., 2015; Rivera-Paleo \& Guzmán, 2019; Weih et al., 2020c). Instead of crossing beams, the M1 method yields merging beams, which is obviously not the correct physical behavior and can lead to large errors in simulations of realistic physical systems (Foucart et al., 2018). We will demonstrate this problem in detail in Sec. 5.2.3, when we present a new method for radiative transport that does not suffer from this problem.

\section{Radiation Wave in the Free-Streaming Regime}

For the next test we evolve a spherically symmetric radiation wave. In order to test our implementation also for non-Cartesian coordinates, we use BHAC's capabilities for setting up a polar grid. The radiation wave is initialized by a constant $J$ in a circular region around the grid center, where the flux is set to $H_{r}=J$. Outside of the inner region, we again set all variables to $10^{-15}$. The top panels of Fig. 5.3 show that the radiation propagates outwards in a radial manner maintaining its spherical symmetry.

Since we use polar coordinates, the energy density is expected to decrease following an inverse square law. We verify this by extracting $J$ along the diagonal of the grid at every time of the simulation. This is shown in the bottom panel of Fig. 5.3 for some representative times. A function proportional to $1 / r^{2}$ is then fitted to the maxima of these cuts, shown as red-dashed line. This fit reveals, that the maximum of the radiation energy density decreases as expected. 


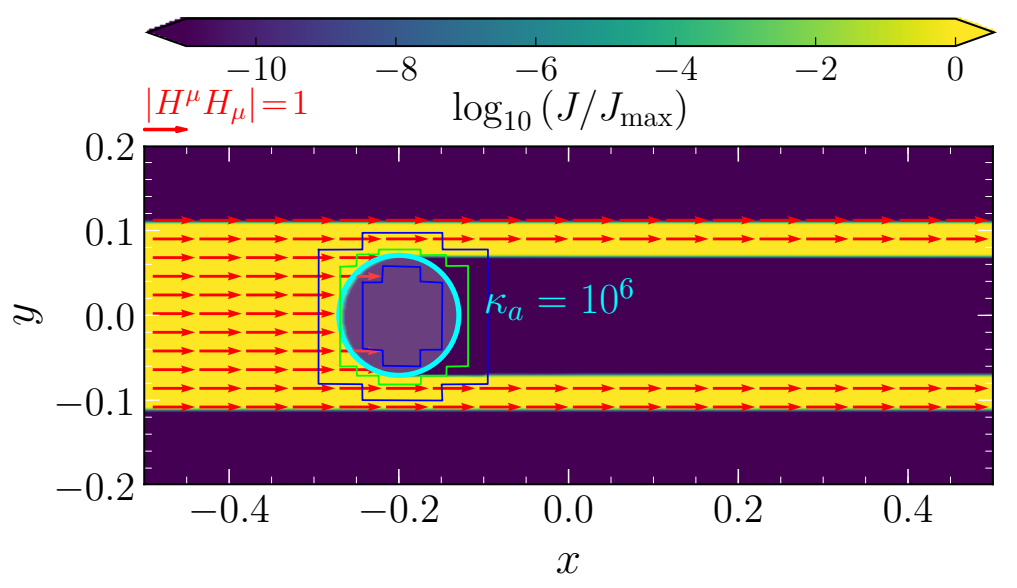

Figure 5.4: Top: Same as Fig. 5.1, but with an absorbing sphere shown by a cyan-colored circle. The colored boxes represent the refinement levels used for this simulation. Reprint of Fig. 5 in Weih et al. (2020c).

\section{Shadow Test}

The previous tests both considered radiation propagating in vacuum. We now verify that also when an absorbing fluid is present, the code performs correctly. We do so not by introducing an actual fluid (the fluid variables are still set to zero), but by choosing a finite and constant value for the absorption opacity, $\kappa_{a}$. We repeat the first beam test, but set $\kappa_{a}=10^{6}$ inside a sphere of radius $r=0.07$ centered at $(x, y)=(-0.2,0)$ (see cyan colored circle in Fig. 5.4). One would expect that the radiation is absorbed by this sphere, so that effectively this scenario describes the beam of a flash light shining on an object resulting in a shadow behind this object. As additional difficulty, we activate two refinement levels within BHAC around the absorbing sphere in order to also test the correct computation of the fluxes at the fine-coarse and coarse-fine interfaces.

Fig. 5.4 shows that the beam correctly casts a shadow behind the sphere. Even more so, the two resulting beams on top and bottom of the sphere remain collimated and only a marginal amount of radiation is found to diffuse inside the region of the shadow. This result also shows that our IMEX works as intended, because the large value chosen for $\kappa_{a}$ leads to a stiff system of evolution equations. We have checked that using an explicit time stepper for the same simulation with the same time step results in an unstable evolution.

\section{Emitting Sphere Test}

The previous tests are all based on our intuition about the behavior of radiation and can only be used to qualitatively verify our implementation. The emitting sphere test also allows for a quantitative verification. This test, first proposed by Smit et al. (1997), takes advantage of a setup whose steady-state solution can be computed analytically from the Boltzmann equation. Specifically, we consider a homogeneous sphere of radius $R$ that is in equilibrium, i.e., $\eta=\kappa_{a}$. Such a high-optical depth sphere can be thought of as an isolated neutron star with a sharp boundary to the surrounding vacuum. 


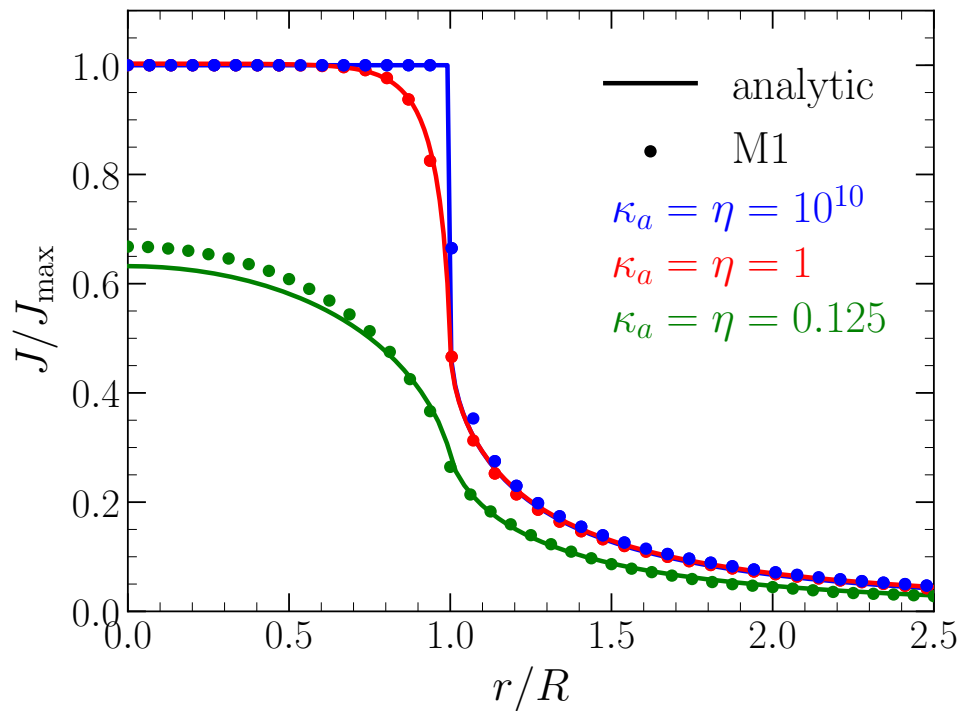

FiguRE 5.5: Emitting sphere test for high (blue), medium (red), and low (green) optical depth. Dots show the equilibrium solution obtained using the M1 scheme. Solid lines show the analytic solution according to Eq. (5.66). Reprint of Fig. 6 in Weih et al. (2020c).

For this setup any radiation field will equilibrate to the distribution

$$
f(r, \mu)=b\left(1-e^{-\kappa_{a} s(r, \mu)}\right),
$$

which depends on the distance to the sphere's center, $r$, and the azimuthal angle, $\theta$, via $\mu:=\cos \theta$. The constant $b$ is simply a scaling factor and

$$
s:= \begin{cases}r \mu+R g(r, \mu), & r<R \wedge-1<\mu<1 \\ 2 R g(r, \mu), & r \geq R \wedge \sqrt{1-R^{2} / r^{2}}<\mu<1\end{cases}
$$

with

$$
g(r, \mu):=\sqrt{1-\frac{r^{2}}{R^{2}}\left(1-\mu^{2}\right)} .
$$

In these spherical coordinates, the zeroth moment can be computed from the distribution function as

$$
J=\frac{1}{2} \int_{-1}^{1} f(r, \mu) \mathrm{d} \mu .
$$

For the simulations we initialize $J=b$ and $H_{r}=0$ inside the sphere and $J=$ $b R^{2} / r^{2}$ and $H_{r}=0.5 b R^{2} / r^{2}$ outside of it. For simplicity we choose $b=1$. We test a low, medium, and high optical depth by setting $\kappa_{a}=\eta=0.125,1,10^{10}$, respectively, inside the sphere. The results are shown as dots on Fig. 5.5. We compare all cases to the analytic solution (solid lines). In all cases the tail $(r>R)$ shows excellent agreement with the analytic solution. Note that this is due to the fact that we run the simulations in three dimension. Despite the spherically symmetric equilibrium, on a Cartesian grid fluxes will propagate also in the angular directions as already remarked in Radice et al. (2013). Indeed, this tail can not correctly be reproduced when running in only one or two dimensions.

For $\kappa_{a}=\eta=10^{10}$ (blue) and $\kappa_{a}=\eta=1$ we also find good agreement inside the high-optical depth sphere and even at the sharp transition to the surrounding vacuum. 

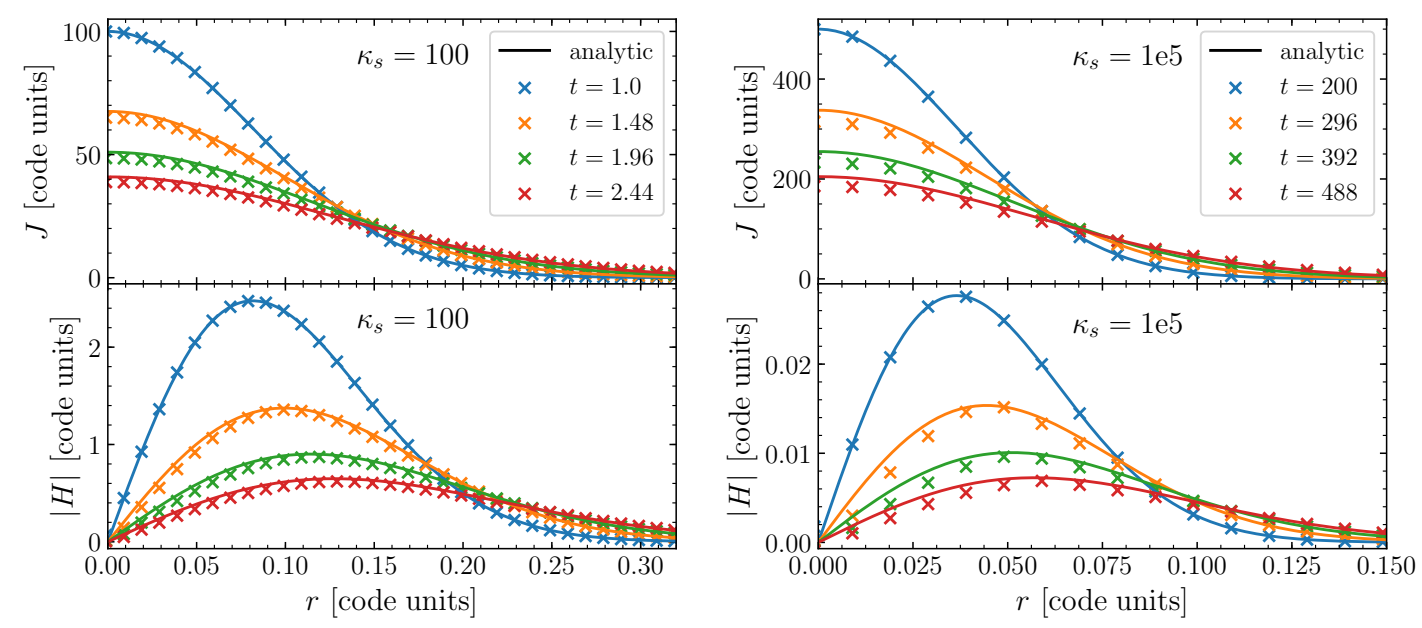

FIgURE 5.6: Diffusion of radiation pulse after four representative times for $\kappa_{s}=100$ (left) and $\kappa_{s}=10^{5}$ (right). Shown is the radiation energy density (top) and the magnitude of the momentum density (bottom). The numerical solution (crosses) is compared to the analytic one (solid lines), i.e., Eqs. (5.67) and (5.68). Reprint of Fig. 7 in Weih et al. (2020c).

This is again only possible due to the IMEX scheme. The case with $\kappa_{a}=\eta=0.125$ (green), on the other hand, shows a discrepancy w.r.t. the analytical solution for $r<R$. This is due to the approximate closure relation; while the closure is exact in the free-streaming regime $(r>R)$ and for high optical depth (red and blue cases), it is not in the intermediate regime. It seems that $\kappa_{a}=\eta=0.125$ falls exactly in this intermediate regime and thus the wrong computation of the second moment leads to an overall wrong equilibrium solution (see also Fig. 2 in Murchikova et al., 2017).

\section{Diffusion Wave}

Having verified emission and absorption, the final reaction to check is scattering. Scattering is particularly difficult to implement since it does not only require the IMEX method, but also the non-local flux corrections discussed in Sec. 5.1.1. Physically, scattering is the dominating process whenever densities are high like in the core of an HMNS. Radiation will only slowly diffuse out of the HMNS and then stream from its surface. In order to ensure that our implementation of the M1 scheme reproduces the correct diffusion rate, we perform the diffusion-wave test as done in Pons et al. (2000). The idea is that a point-like radiation pulse on top of a dense homogeneous fluid will diffuse through this fluid isotropically. The spherically symmetric time-dependent solution in $d$ dimensions is

$$
\begin{aligned}
J(r, t) & =\left(\frac{\kappa_{s}}{t}\right)^{d / 2} \exp \left(\frac{-3 \kappa_{s} r^{2}}{4 t}\right), \\
H(r, t) & =\frac{r}{2 t} J .
\end{aligned}
$$

This solution only holds when the scattering opacity, $\kappa_{s}$, does not change, i.e., only in an idealized scenario where the radiation does not backreact onto the fluid. This can easily be done numerically by turning off this backreaction and setting a constant value for $\kappa_{s}$ throughout the simulation.

We perform two simulations in two dimensions $(d=2)$ with a moderate opacity of $\kappa_{s}=100$ and a high one of $\kappa_{s}=10^{5}$. We use a Cartesian grid of size $x, y \in[-0.5 ; 0.5]$ with $100^{2}$ grid cells. Since we can not initialize a point-like pulse numerically, we 

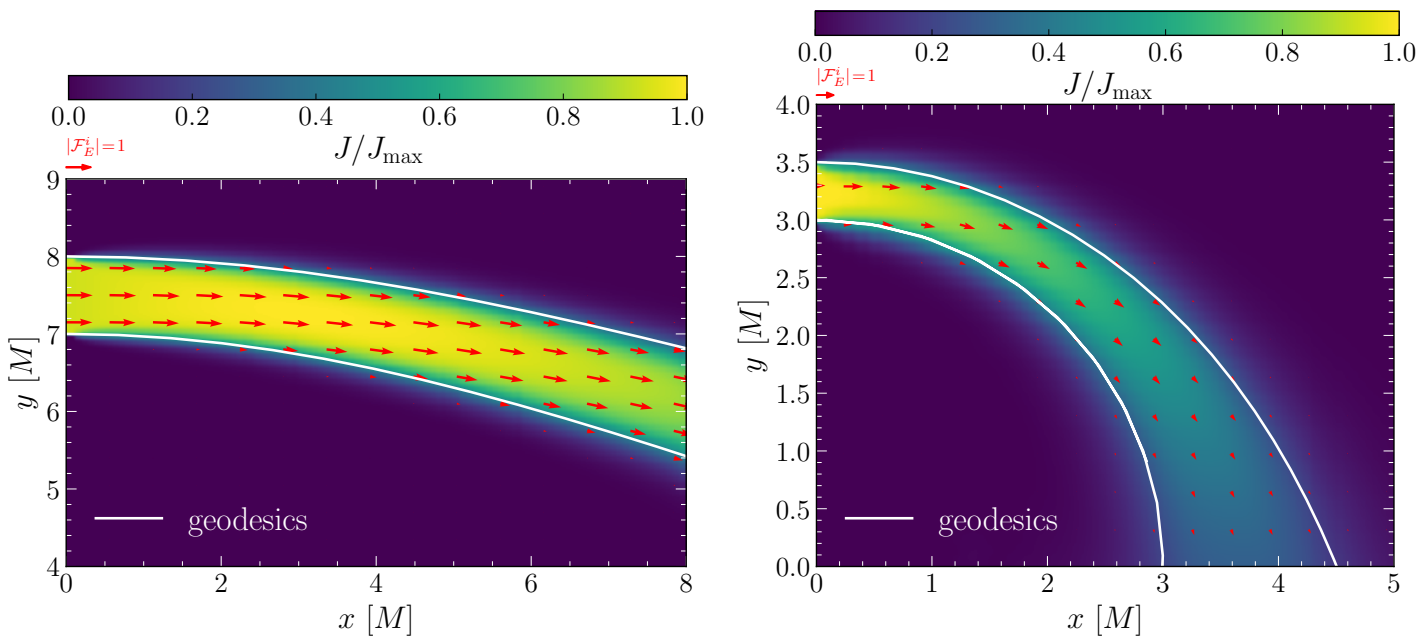

Figure 5.7: Same as Fig. 5.1, but in a curved spacetime with a black hole of mass $M$ located at $(x, y)=(0,0)$. White lines show the geodesics for the top and bottom edge of the beams. Shown is the final configuration for a beam injected at $y \in[7 M ; 8 M]$ (left) and at $y \in[3 M ; 3.5 M]$ (right). Reprint of Figs. 9 and 10 in Weih et al. (2020c).

instead use Eqs. (5.67) and (5.68) as initial data with $t=1$ for the moderate and $t=200$ for the high opacity simulation.

Fig. 5.6 shows the numerical results for $\kappa_{s}=100$ (left) and $\kappa_{s}=10^{5}$ (right) in comparison to the analytical solution. The latter case deserves special attention: despite the IMEX scheme guaranteeing a stable numerical evolution for such a high opacity, and also the correct pressure tensor in the limit of $\kappa_{s} \rightarrow \infty$ implemented in the closure, the M1 method is not expected to yield the correct diffusion behavior (see e.g., Pons et al., 2000; O'Connor, 2015). This is due to the fluxes, which only include first-order spatial derivatives instead of the second-order derivatives of a diffusion equation. The main reason for why Fig. 5.6 shows good agreement with the analytic solution nonetheless is the correction to the flux terms discussed in Sec. 5.1.1. Indeed, we find for the weight in Eq. (5.37) $a \approx 0.01$, i.e., the asymptotic correction's contribution to $\mathcal{F}_{E, \text { corr }}^{i}$ is $99 \%$. The small discrepancy between the numerical and analytic solution is then a combination of the artificial diffusion present in all gridbased codes and the remaining $1 \%$ of the flux term.

\section{Curved Beam Test}

The previous tests are all performed in the absence of gravity, i.e., in a flat spacetime. The introduction of gravity drastically complicates the evolution equations and their implementation. Due to this complexity the only standard test in a curved spacetime is that of a curved beam. This test resembles the first one in the sense that a beam of radiation is shot into the right from the left boundary. However, this time the spacetime is that of a non-rotating black hole of mass $M$ located at the grid's origin, whose metric is expressed in Cartesian Kerr-Schild coordinates.

More specifically, we inject the beam via setting $J=1$ (note that in the absence of fluid motion we still have $J \equiv E$ ) for $y \in[7 M ; 8 M]$ for the two left-most grid cells in $\mathrm{x}$-direction. In the same region we ensure free-streaming via the condition for the optically thin limit, i.e.,

$$
H^{\mu} H_{\mu}=J^{2} .
$$



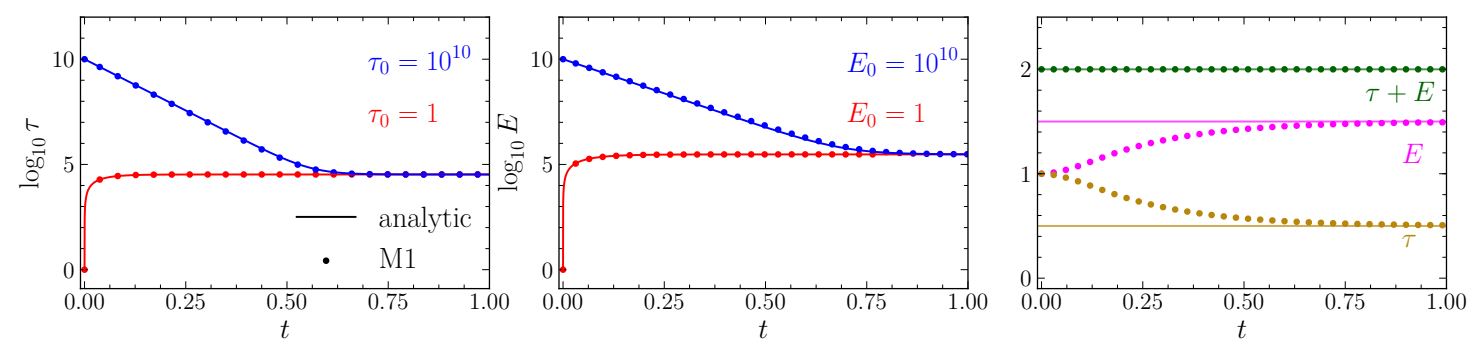

FIGURE 5.8: Left: Evolution of the rescaled total fluid energy density when the radiation energy is held constant for two initial energies. The solid lines show the analytic solution according to Eq. (5.70), while the dots show the numerical results obtained with the M1 scheme. Middle: Same as the left panel but for $E$ with $\tau$ being held constant. Right: Coupled evolution of $\tau$ (golden) and $E$ (magenta) without injecting energy into the system. The green dots show that the total energy is constant throughout the evolution. Adapted from Fig. 8 in Weih et al. (2020c).

We also ensure that the beam is initially propagating parallel to the x-axis, i.e., $\mathcal{F}_{E}^{y}=$ $0=\mathcal{F}_{E}^{z}$. Both conditions are sufficient to initialize the momentum density.

The simulation then yields a beam that curves around the central black hole, which is shown in Fig. 5.7. The top and bottom edge of the beam is expected to follow geodesics. The white lines in the same figure show that this is the case and only a small amount of radiation diffuses outside of the area enclosed by these geodesics.

We repeat the same test for a beam closer to the black hole, i.e., with the beam's inner edge at the photon ring, by initializing the region for $y \in[3 M, 3.5 M]$. The diffusion is much larger in this case. The total energies of the initial configuration (at $x=0$ ) and the final one (at $y=0)$ differ by $11.9 \%$.

\section{Fluid-Radiation Coupling Test}

The final test is for the coupling between radiation and fluid, i.e., the coupling between FRAC and BHAC, which has so far been neglected. We use the test presented in Turner \& Stone (2001); Melon Fuksman \& Mignone (2019), which consists of a static fluid and radiation field. This can be achieved by either removing the flux terms from the evolution equations or by initializing $F_{i}=v_{i}=0$ together with a spatially homogeneous value for the radiation energy density, $E$, and the rescaled fluid energy density, $\tau$. Without magnetic fields and scattering and returning to a flat spacetime the coupled evolution of the fluid and radiation then simplifies to

$$
\begin{aligned}
\partial_{t} \tau & =-G_{0}=-\eta+\kappa_{a} E, \\
\partial_{t} E & =G_{0}=\eta-\kappa_{a} E .
\end{aligned}
$$

The second equality follows from Eq. (5.18) and the fact that $J \equiv E$ and $H^{\mu} \equiv F^{\mu}$ in the absence of fluid motion.

A particularly simple - yet unphysical - system is obtained when choosing $\kappa_{a}=$ const. and $\eta=\kappa_{a} \tilde{\eta} \tau$, where $\tilde{\eta}$ takes a constant value. In fact, this choice for $\eta$ is not too far from a realistic emissivity, which according to Kirchhoff's law is directly proportional to $\kappa_{a}$ and also depends on the fluid variables (typically density and temperature, but $\tau$ in this case). Plugging these parameters in Eq. (5.70) and holding 
$E$ constant throughout the evolution yields

$$
\tau(t)=\left(\tau_{0}-\frac{E}{\tilde{\eta}}\right) \exp \left(-\kappa_{a} \tilde{\eta} t\right)+\frac{E}{\tilde{\eta}},
$$

where $\tau_{0}:=\tau(t=0)$. Likewise, for constant $\tau$, the solution of the radiation according to Eq. (5.71) is

$$
E(t)=\left(E_{0}-\tilde{\eta} \tau\right) \exp \left(-\kappa_{a} t\right)+\tilde{\eta} \tau,
$$

where $E_{0}:=E(t=0)$.

For both cases (i.e., $\tau=$ const. or $E=$ const.) we perform a simulation with a dominating fluid and a dominating radiation field, respectively. Specifically, for $\tau=$ const. we keep $E=10^{5}$ throughout the simulation and perform two tests with $\tau_{0}=1$ and $\tau_{0}=10^{10}$, respectively. The results are shown in the left panel of Fig. 5.8. In both cases $\tau$ can be seen to equilibrate to the correct steady-state solution. For $\kappa_{a}=1$ and $\tilde{\eta}=3$ this final solution is $\tau(t \rightarrow \infty)=1 / 3 \times 10^{5}$ (cf., Eq. (5.70) for $\left.G_{0} \rightarrow 0\right)$.

The second case, i.e., with a constant $\tau=10^{5}$, is shown in the middle panel. Again we choose two different initial configurations simulating a dominating fluid and radiation field, respectively. This time, Eq. (5.71) yields for the same choice of $\kappa_{a}$ and $\tilde{\eta}$ an equilibrium value of $E(t \rightarrow \infty)=3 \times 10^{5}$. The simulation results are in both cases in excellent agreement with this value.

Finally, we also perform a coupled simulation without holding one of the evolved variables constant. In other words, we do not inject energy into the system and thus expect the energy to be conserved throughout the simulation. Independent of $\kappa_{a}$ and $\tilde{\eta}$ the total energy, $\tau+E$, remains at the constant value $\tau+E=\tau_{0}+E_{0}=$ $1+1=2$. The green dots in the right panel in Fig. 5.8 proves that this is indeed the case. Furthermore, the coupled system of Eqs. (5.70) and (5.71) reveals that for $G_{0}(t \rightarrow \infty)=0$ the equilibrium values are $E(t \rightarrow \infty)=2 \tilde{\eta} /(1+\tilde{\eta})=3 / 2$ and $\tau(t \rightarrow \infty)=2 /(1+\tilde{\eta})=1 / 2$. Again the numerical results match this expectation.

\subsubsection{The Radiative Michel Solution}

The previous tests verify that all parts of the new radiation code FRAC and its coupling to BHAC work correctly. Having tested all terms separately, we now use everything together for an astrophysically realistic scenario. Specifically, in this section we present simulation results from spherically symmetric accretion onto a black hole.

This problem was first studied by Bondi (1952) in Newtonian physics. Later on, Michel (1972) extended it to a general-relativistic context, but still neglecting radiation. In reality, the accreted matter will produce photons close to the horizon, whose emission should provide an observable signature. Also magnetic fields will play a role in the dynamics of the accreted matter and in determining the correct accretion efficiency. Many works have studied this problem with different levels of realism beginning with simplified approaches for radiation (see e.g., Vitello, 1978; Begelman, 1978; Gillman \& Stellingwerf, 1980). More realistic simulations using selfconsistent methods for radiative transport come from Nobili et al. (1991) and Zampieri et al. (1996), which are the only simulations to include Comptonization. Sądowski et al. (2013); McKinney et al. (2013) probably contributed the most sophisticated simulations in terms of radiative transport making use of the Levermore closure. While Fragile et al. (2012); Roedig et al. (2012) also used a self-consistent M1 scheme, their closure was only accurate in the optically thick limit, i.e., close to the central black hole. Consequently, they could not obtain reliable results far away from the 


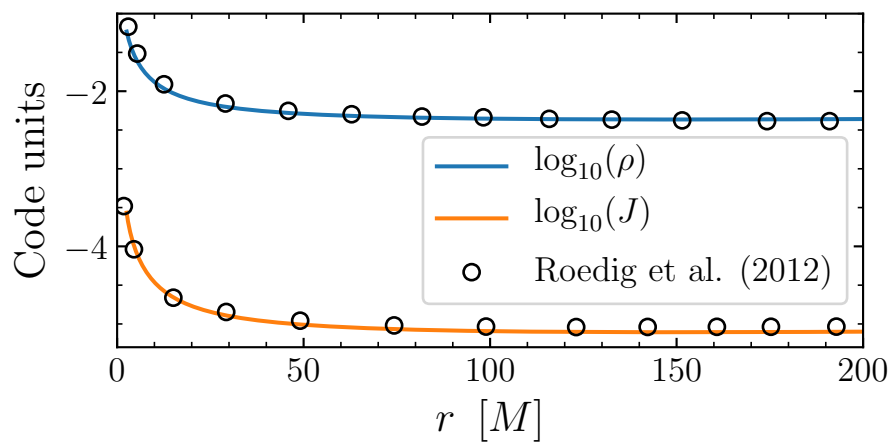

FiguRE 5.9: Equilibrium solution for spherically symmetric accretion onto a non-rotating black hole for simplified opacities (see main text). The solid lines compare the fluid rest-mass density (blue) and the radiation energy density in the fluid frame (orange) to the results obtained by Roedig et al. (2012) (circles). Reprint of Fig. 11 in Weih et al. (2020c).

black hole, which is exactly the region where an observable luminosity should be extracted. The results presented in this section are also obtained from a closure (the M1 closure described in Sec. 5.1.1) that is accurate in both, optically thick and thin limit. The results can therefore be used to find a suitable relation that describes the observable luminosity in terms of the accretion rate.

\section{Uniformly Absorbing Matter}

We start by considering a somewhat simplified setup for the radiative Michel problem. This is a scenario first introduced by Roedig et al. (2012), in which the opacities are assumed to be constant over the entire grid. In particular, we set $\kappa_{a}=10^{15}$ and $\kappa_{s}=0$, while the emissivity is that of a black body, i.e.,

$$
\eta=4 \pi \kappa_{a} \frac{\sigma_{\mathrm{SB}}}{c} T^{4}
$$

where $T$ is the fluid temperature and $\sigma_{\mathrm{SB}}$ the Stefan-Boltzmann constant. The numerical value of this constant is set to $\sigma_{\mathrm{SB}}=0.0479$. This results in a unit system that is the same as the one described in Zanotti et al. (2011) and thus allows for an easy comparison of the results. Note that the choice of $\kappa_{a}$ and $\kappa_{s}$ is physically unrealistic. However, it allows us to test all terms in our code together and compare the results to that of Roedig et al. (2012). Furthermore, the high value of $\kappa_{a}$ is yet another test for the implemented IMEX method, for which we use a CFL number of 0.2 .

The spacetime is fixed to be that of a non-rotating black hole with mass $M=$ $2.5 M_{\odot}$. The fluid is treated as a perfect one with an ideal-gas EOS with adiabatic index $\gamma=4 / 3$. The one-dimensional grid is also the same as in Roedig et al. (2012): it covers a range of $2.5<r / M<200$ with 300 cells, where $r$ is the radial coordinate of the underlying Boyer-Lindquist coordinates.

The equilibrium solution of this simplified accretion problem are shown in Fig. 5.9 for the fluid rest-mass density, $\rho$, and the radiation energy-density in the fluid frame, $J$. The circles compare the results of Roedig et al. (2012) to those obtained with FRAC. Obviously, the agreement is quite well, which is to be expected for the following reason: despite the different closures, the constant value of $\kappa_{a}=10^{15}$ ensures that the entire domain is in the optically thick limit. In this limit the M1 closure implemented in FRAC coincides with the simplified closure in Eq. (5.32), which is the one used in 


\begin{tabular}{lcccc} 
Model & $\dot{M} / \dot{M}_{\text {Edd }}$ & $T_{0}$ & $1 / \beta_{\mathrm{rad}}$ & $L / L_{\text {Edd }}$ \\
\hline E1T6 & 1.0 & $10^{6}$ & $1.2 \times 10^{-4}$ & $2.33 \times 10^{-8}$ \\
E10T5 & 10.0 & $10^{5}$ & $1.2 \times 10^{-7}$ & $6.62 \times 10^{-7}$ \\
E10T6 & 10.0 & $10^{6}$ & $1.2 \times 10^{-4}$ & $2.65 \times 10^{-6}$ \\
E10T7 & 10.0 & $10^{7}$ & $1.2 \times 10^{-1}$ & $6.41 \times 10^{-6}$ \\
E100T6 & 100.0 & $10^{6}$ & $1.2 \times 10^{-4}$ & $2.01 \times 10^{-4}$ \\
\hline
\end{tabular}

TABLE 5.1: Different models and their parameters for the simulations of the radiative Michel problem. The runs are the same ones as in Fragile et al. (2012); Sạdowski et al. (2013). The final luminosities are extracted at $r=1000 \mathrm{M}$. The profiles for the radiation and fluid quantities of these runs are shown in Fig. 5.10. Reprint of Tab. 1 in Weih et al. (2020c).

Roedig et al. (2012).

While the latter is limited to this unrealistic scenario, our variable closure allows a more realistic simulation of the radiative Michel problem.

\section{Realistic Radiation}

For a more realistic setup we follow the prescription by Sądowski et al. (2013). Instead of having constant opacities, the energy-averaged absorption opacity is that of thermal bremsstrahlung, i.e., (Rybicki \& Lightman, 1986)

$$
\kappa_{a}=6.1 \times 10^{22} T_{\mathrm{K}}^{-3.5} \rho_{\mathrm{cgs}} \mathrm{cm}^{-1},
$$

where the temperature is in Kelvin and the density in $\mathrm{g} \mathrm{cm}^{-3} 5$. Scattering is modeled via Thomson scattering given by

$$
\kappa_{s}=0.4 \rho_{\mathrm{cgs}} \mathrm{cm}^{-1} .
$$

The accreted matter is initialized as freely infalling material, for which the restmass density reads

$$
\rho=\frac{\dot{M}}{4 \pi r^{2} v},
$$

where $\dot{M}$ is the accretion rate and the magnitude of the free-fall three-velocity is $v:=\sqrt{v_{i} v^{i}}=\sqrt{2 M / r}$. Assuming an inwards radial motion of the fluid implies for the components of the three-velocity that

$$
v^{r}=-\sqrt{v^{2} / g_{r r}}, \quad v^{\phi}=0=v^{\theta},
$$

where $g_{r r}$ is the radial component of the four-metric, for which we use modified KerrSchild polar coordinates as described in Porth et al. (2017). For the fluid the initial specification of either the temperature or the pressure is required. We choose to

\footnotetext{
${ }^{5}$ Typically, simulations of accretion problems are scale-invariant, which means that for $c=G=1$ all quantities can be expressed in terms of the black hole mass, $M$. This is possible because the accreted material is assumed to be much lighter than the black hole, so that it does not contribute to the curvature of the spacetime. While this assumption is still true in the presence of radiation, the simulation loses its scale-invariance. This is because the coupling with the radiation requires an actual scale that determines the mass of, e.g., a proton compared to that of the plasma torus. This mass than enters in the computation of the opacities and the emissivity. For more details see also Appendix A on the units used for this simulation.
} 
initialize the pressure as

$$
p=\frac{k_{\mathrm{B}} T_{0}}{\mu m_{\mathrm{p}}} \frac{\rho^{\gamma}}{\rho_{0}^{1-\gamma}},
$$

where we assume a polytropic EOS of the form $p \propto \rho^{\gamma}$ with adiabatic index $\gamma . T_{0}$ and $\rho_{0}$ are the temperature and density, respectively, at a fiducial radius $r_{0}$ and $\mu=0.5$ is the mean molecular weight of fully ionized hydrogen with proton mass $m_{\mathrm{p}}$. Note that this setup is equivalent to that of Fragile et al. (2012); McKinney et al. (2013), who initialize the temperature instead of the pressure. The adiabatic index is determined by the initial ratio of fluid to radiation pressure, $\beta_{\text {rad }}$, via

$$
\gamma=1+\frac{2}{3}\left(\frac{\beta_{\mathrm{rad}}+1}{\beta_{\mathrm{rad}}+2}\right)
$$

and is constrained to be $4 / 3<\gamma<5 / 3$.

The radiation field is initialized as

$$
E=3 p / \beta_{\mathrm{rad}}
$$

and the radiation momentum density is initially set to zero. Since we are interested in finding an equilibrium state, the initial configuration of the radiation can in principle be arbitrary and we have verified that a different initialization still leads to the same result.

This setup has five free parameters in total, i.e., the black hole mass, $M$, the accretion rate, $\dot{M}$, the temperature, $T_{0}$, the fluid-to-radiation pressure ratio, $\beta_{\mathrm{rad}}$, and the fiducial radius, $r_{0}$. Fixing $r_{0}=2 \times 10^{4} M$ and $M=3 M_{\odot}$ leaves $T_{0}, \dot{M}$, and $\beta_{\mathrm{rad}}$ as parameters that determine the properties of the accreted matter. We vary these parameters to obtain five different simulations that correspond to the ones in Sądowski et al. (2013) and are summarized in Tab. 5.1.

All these simulations are performed in two dimensions on a polar grid that ranges from $1.1 r_{\mathrm{S}}$ to $10^{4} r_{\mathrm{S}}$ in the radial direction, with $r_{\mathrm{S}}=2 M$ being the Schwarzschild radius, and from 0 to $\pi / 2$ in the angular direction. We use a resolution of $600 \times 40$ grid-points and apply outflow boundary conditions at the outer edge of the domain.

The luminosity is computed as

$$
L=4 \pi r^{2} F,
$$

where $F=\sqrt{F_{i} F^{i}}$ and the extraction radius is $r=10^{3} \mathrm{M}$. We have verified that a somewhat smaller or larger extraction radius only changes our results marginally. Furthermore, using the momentum-density in the Eulerian frame or the fluid frame leads to the same luminosity, because in the far-zone the fluid velocity approaches zero and thus $F \approx H$.

Using FRAC coupled to BHAC yields the results shown in Fig. 5.10. This figure shows the equilibrium solution for the fluid rest-mass density, temperature, radiation energy density, and radiation momentum density. For all cases, we observe smooth profiles. This is in contrast to the results reported in Fragile et al. (2012), where the profiles far away from the black hole oscillate. As already described in Sagdowski et al. (2013); McKinney et al. (2013) this is due to the more sophisticated closure relation, which in our case is also accurate in the optically thin limit, i.e., far away from the black hole.

The profiles of the radiation field can be fitted by a simple power-law of the form $J(H) \propto r^{-a}\left(r^{-b}\right)$ with $a \sim 1.91(b \sim 1.78)$. Comparing to the same results obtained by Sạdowski et al. (2013); McKinney et al. (2013) shows that the radiation profiles 

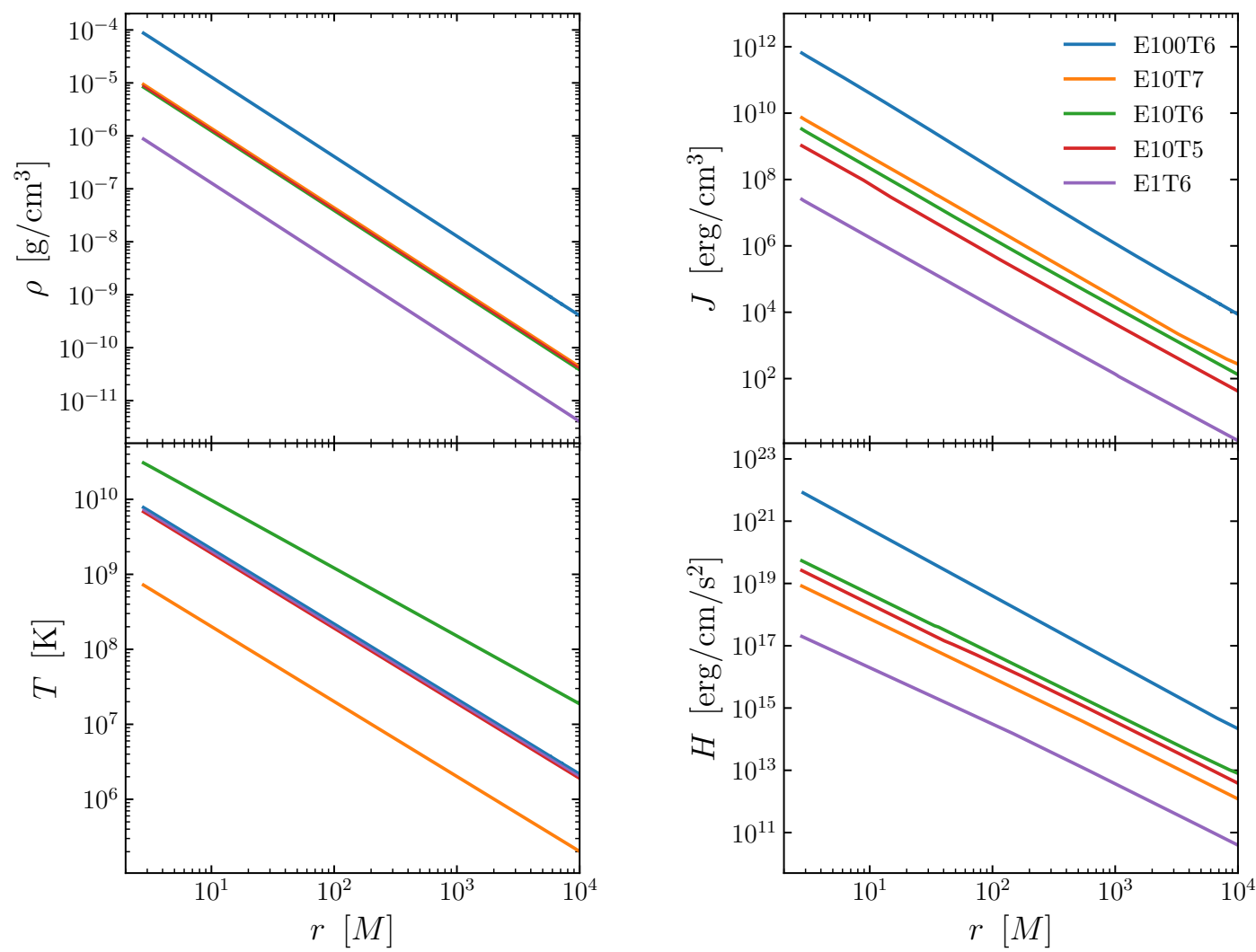

FigURE 5.10: Equilibrium profiles of the fluid and radiation quantities for the radiative Michel accretion problem. Different colors correspond to the different models listed in Tab. 5.1. Reprint of Fig. 12 in Weih et al. (2020c).

presented here are consistently lower by a factor of $\sim 4$. The same is true for the extracted luminosities. A possible reason for this might be the different implementation of the M1 scheme. While FRAC follows the moment formalism and specifically the implementation detailed in Shibata et al. (2011), the radiation in Sagdowski et al. (2013); McKinney et al. (2013) is implemented under the assumption that there is always a frame, in which the radiation flux vanishes. The radiation quantities are then computed in this so-called radiation frame, which does not exist in our implementation. Roedig et al. (2012), on the other hand, follows the same implementation as we do and indeed Fig. 5.9 shows that our results agree very well with those of Roedig et al. (2012). Nevertheless, considering that the profiles in Fig. 5.10 vary over $\sim 7$ orders of magnitude, it is still reassuring that the qualitative behavior is the same as in Sagdowski et al. (2013); McKinney et al. (2013) and that the quantitative agreement is well within the same order of magnitude.

We run additional simulations so that we cover a total of 20 runs, whose parameters are $T_{0}=10^{4}, 10^{5}, 10^{6}, 10^{7} \mathrm{~K}$ and $\dot{\mathcal{M}}:=\dot{M} / \dot{M}_{\text {Edd }}=1,3,10,30,100$, where the Eddington accretion rate is

$$
\dot{M}_{\mathrm{Edd}}:=\frac{L_{\mathrm{Edd}}}{c^{2}}=\frac{4 \pi G M m_{\mathrm{p}}}{c \sigma_{\mathrm{T}, \mathrm{e}}} \approx 1.39 \times 10^{17} \frac{M}{M_{\odot}} \frac{\mathrm{g}}{\mathrm{s}}
$$

with the Thomson cross-section of electrons $\sigma_{\mathrm{T}, \mathrm{e}}=6.65 \times 10^{-25} \mathrm{~cm}^{2}$ (see Appendix A for details on units). In Fig. 5.11 we show these runs in the $\mathcal{L}-\dot{\mathcal{M}}$ plane, where $\mathcal{L}:=L / L_{\mathrm{Edd}}$ is the extracted bolometric luminosity normalized to the Eddington luminosity. 


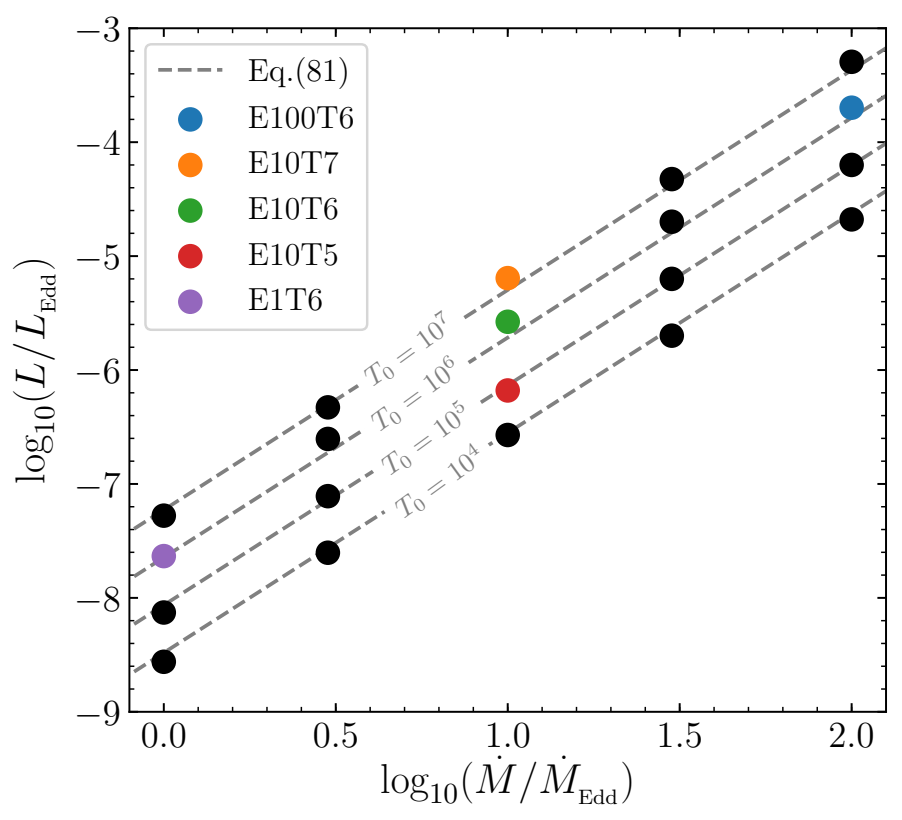

Figure 5.11: Normalized luminosity $\mathcal{L}:=L / L_{\text {Edd }}$ over normalized accretion rate $\dot{\mathcal{M}}:=$ $\dot{M} / \dot{M}_{\text {Edd }}$. The colored dots correspond to the same models as in Fig. 5.10 and Tab. 5.1, while the black ones are additional runs. The dashed lines show the relation (5.84) for different values of $T_{0}$. Reprint of Fig. 13 in Weih et al. (2020c).

The simulations cover a large enough portion of the parameter space for recognizing a clear dependence of $\mathcal{L}$ on $T_{0}$ and $\dot{\mathcal{M}}$. Specifically, we can apply a fit of the form

$$
\log _{10}\left(\mathcal{L}\left(\dot{\mathcal{M}}, T_{0}\right)\right)=a \log _{10}(\dot{\mathcal{M}})+b
$$

with fitting parameters $a$ and $b$. As evident from Fig. 5.11 these fitting parameters depend on $T_{0}$, i.e., $a=a\left(T_{0}\right)$ and $b=b\left(T_{0}\right)$. The slope $a$ is practically the same for all values of $T_{0}$ (the variation is smaller than the associated fitting error), so that for all $T_{0}$ we can write $a=1.930 \pm 0.067$. For $b$ we find a roughly linear dependence, i.e., $b=(0.418 \pm 0.026) \log _{10}\left(T_{0}\right)-(10.154 \pm 0.144)$. Using Eq. (5.84), the accretion efficiency can be obtained as

$$
\begin{aligned}
\epsilon & :=\mathcal{L} / \dot{\mathcal{M}}=10^{b / a} \mathcal{L} \approx 5.481 \times 10^{-6} T_{0}^{0.217}\left(\frac{L}{L_{\mathrm{Edd}}}\right)^{0.482} \\
& =7.41 \times 10^{-7}\left(\frac{T_{0}}{10^{6} \mathrm{~K}}\right)^{0.22}\left(\frac{L}{L_{\odot}}\right)^{0.48}\left(\frac{M}{M_{\odot}}\right)^{0.48} .
\end{aligned}
$$

This expression can be used to relate the observed luminosity to the either the mass of the black hole or the properties of the surrounding plasma.

\section{The Perturbed Radiative Michel Solution}

Going one step further, we analyze whether the equilibrium solutions obtained previously are stable equilibria (see also Waters et al., 2020, for a discussion on stability for the purely hydrodynamic Michel solution). For doing so, a perturbation in the 


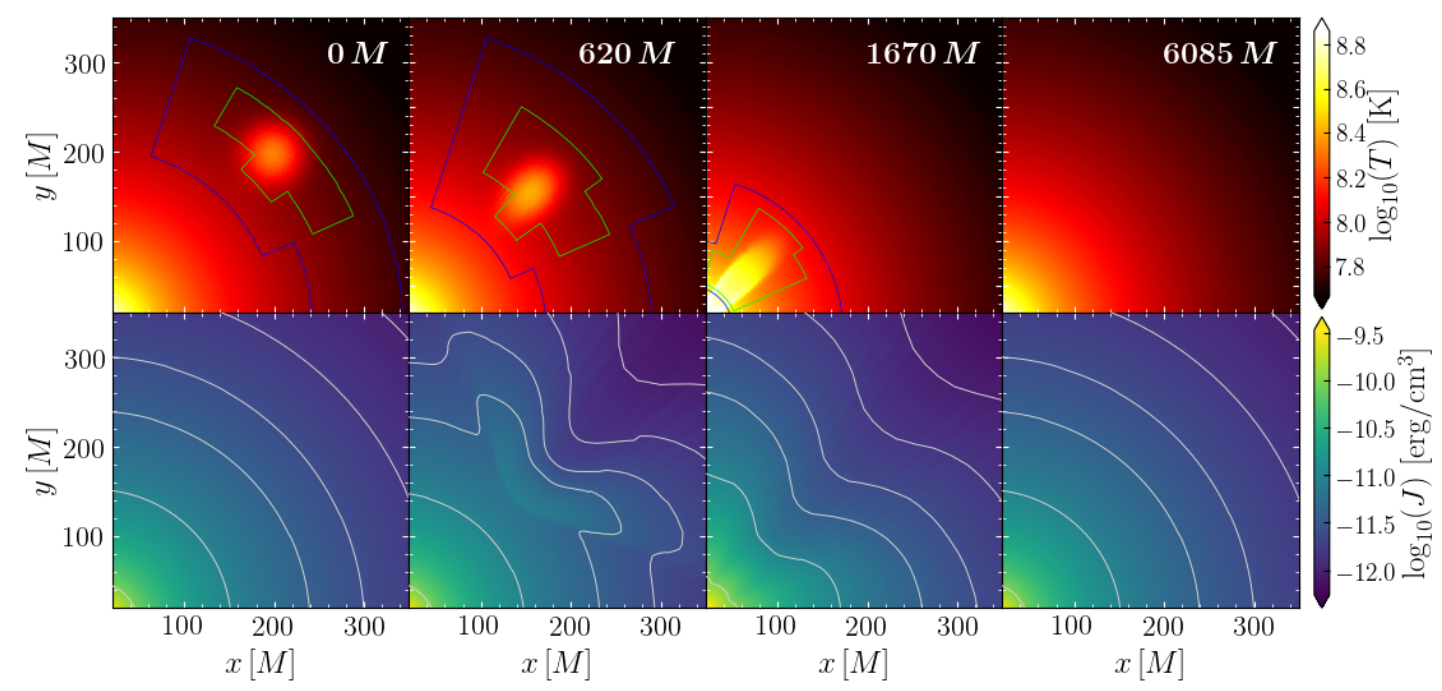

FIGURE 5.12: Evolution of the perturbed radiative Michel solution. Shown is the fluid temperature (top) and the radiation energy density in the fluid frame (bottom). From left to right the different panels show snapshots for an increasing evolution time beginning with the initial configuration on the left. Colored boxes in the top panels show the additional refinement levels (note that the asymmetry is due to the perturbation not being aligned with BHAC's block structure). In the bottom panels contour lines are shown for $\log _{10}(J)=$ $[-12,-11.8,-11.6,-11.4,-11,-10]$. Reprint of Fig. 14 in Weih et al. (2020c).

temperature is introduced modeled via a bivariat Gaussian, i.e.,

$$
\Delta T=A \exp \left(\frac{\left(x-x_{0}\right)^{2}}{\sigma^{2}}+\frac{\left(y-y_{0}\right)^{2}}{\sigma^{2}}\right)
$$

where the local size of the symmetric perturbation is $\sigma=800$, the amplitude $A=2.5$ and its central location is given by $x_{0}=y_{0}=200 \mathrm{M}$. While the pressure and density distributions are immediately affected by this perturbation via the EOS, the radiation field remains initially unaltered and only changes during the simulation through the coupling with the fluid.

The numerical setup remains the same as previously, but with now two levels of mesh-refinement around the perturbation. The left panel in Fig. 5.12 shows the temperature (top) and radiation energy density (bottom) for the initial configuration in the left-most panel. The refinement levels are shown as colored boxes.

The same figure shows the overall evolution of this perturbed system: immediately when starting the simulation, the spherically symmetric perturbation is deformed and dragged towards the black hole. At the same time the radiation field deviates from its initial spherical symmetry due to the increased emissivity and opacities at the region of increased temperature. The second panel from the left shows that the radiation density in front of the temperature hot-spot is increased and falls towards the black hole. Behind the hot-spot, on the other hand, there is a sink in radiation energy, whose cause is similar to the shadowing effect discussed in Sec. 5.1.2. The left panel of Fig. 5.13 shows the corresponding radiation luminosity extracted at $r=10^{3} \mathrm{M}$ at an angle $\theta=\pi / 4$ (diagonally). It can be seen that it drops for $t=1000-2000 \mathrm{M}$, which corresponds to the moment when this outwards propagating region of decreased energy reaches the extraction radius. At the same time, the region of increased $J$ falls towards the black hole, just like the temperature hot-spot itself does. When this excess energy reaches the horizon, it forms a ring of increased radiation energy around the black hole, which then propagates radially outwards in a spherically symmetric 

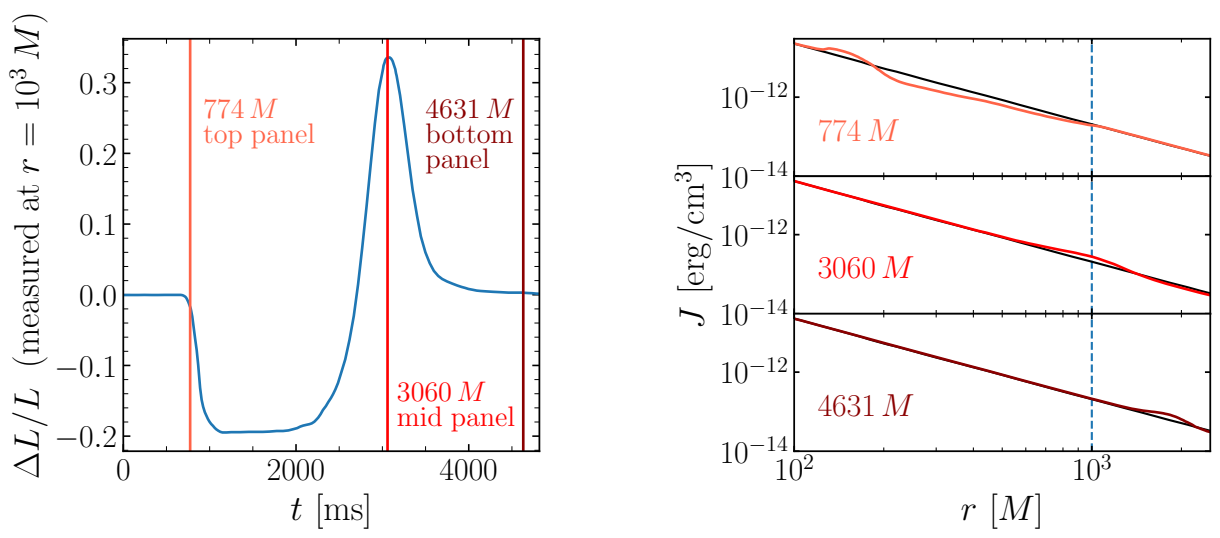

Figure 5.13: Left: Change in luminosity over time for the perturbed radiative Michel solution. Vertical lines mark three representative times, for which the diagonal profiles $(\theta=\pi / 4)$ of $J$ are shown in the right panel. Right: Profiles of the radiation field for three representative times. The vertical dashed line corresponds to the location, where the luminosity of the left panel is measured. Reprint of Fig. 15 in Weih et al. (2020c).

manner. This is the reason for the peak of the luminosity shown in Fig. 5.13 at $t \sim 3060 \mathrm{M}$. The outwards propagating increased energy density can also be seen in the right panel of the same figure, which shows the diagonal profile of $J$ at three different times. Finally, after the temperature hot-spot is completely absorbed by the black hole and after the excess radiation energy leaves the grid, both the radiation and fluid return to their initial equilibrium. This is proof for the radiative Michel solution being non-linearly stable under perturbations of the radiation field. 


\subsection{Beyond Moments: the Lattice Boltzmann Method}

Despite the M1 scheme presented in the previous section being one of the most widely used methods for radiative transport in numerical astrophysics, it has a number of intrinsic deficiencies. Some of these problems are connected to the gray approximation, which can in principle be removed by using a frequency-dependent M1 scheme (Obergaulinger et al., 2014; O'Connor, 2015; Anninos \& Fragile, 2020). Another source of error is connected to the closure relation, which can be revealed by comparison with more sophisticated schemes (e.g., with MC methods in Foucart et al. (2018) or a direct Boltzmann solver in Sumiyoshi et al. (2020)). These more sophisticated schemes, however, suffer from a high computational cost and are unfeasible for three-dimensional simulations as required for binary neutron stars. Consequently, there seems to be a gap between the approximate, but affordable, M1 method and the accurate, but more expensive methods.

We aim to fill this gap by introducing the new Lattice Boltzmann (LB) method for radiative transport. This method is based on classical LB, which is commonly used in computational fluid dynamics (Benzi et al., 1992; Krüger et al., 2017; Succi, 2018), but also for many other disciplines like biology and material science (Succi, 2008). In this section we show the extension of this classical LB method to the case of radiative transport, which has been published in Weih et al. (2020a). This extension is done with a focus on high-energy astrophysics, so that the final method will be applicable in such scenarios. A proof-of-concept simulation of relativistic jets presented in Sec. 5.2.4 demonstrates this. Beforehand, we derive the new method in Sec. 5.2.2 and verify its implementation in Sec. 5.2.3. The latter section uses a similar set of standard tests as applied for the M1 method in Sec. 5.1.2 allowing for a detailed comparison between the two methods. We start by quickly introducing the classical LB method in the following subsection 5.2.1. Note that whenever the term "classical" is used explicitly, it refers to the original LB method that is used for hydrodynamics and has nothing to do with radiation. Nevertheless, it is important to first have an understanding of this classical method before reading the derivation of the LB method for radiative transport in Sec. 5.2.2.

\subsubsection{Classical Lattice Boltzmann in a Nutshell}

This subsection only gives a brief overview of the classical LB method introducing the relevant concepts for the remainder of the section. A detailed introduction can be found in Succi et al. (2010); Krüger et al. (2017); Succi (2018). While introducing special relativity later on for the radiative version of LB, in this subsection we only touch upon the Newtonian case for simplicity. An excellent introduction to specialrelativistic LB can also be found in Gabbana et al. (2020). It will be useful for the remainder of this section to write vectors explicitly in boldface, i.e., instead of components $v^{i}$ from here on we write the entire three-vector $\boldsymbol{v}$.

The starting point is once again the Boltzmann equation, this time written in Newtonian form for particles of mass $m$ without external sources

$$
\left(\frac{\partial}{\partial t}+\frac{p}{m} \cdot \nabla\right) f=\mathcal{C}(f)
$$


where the particle distribution $f(\boldsymbol{r}, \boldsymbol{v}, t)$ depends on space, time, and the microscopic velocity $\boldsymbol{v}=\boldsymbol{p} / \mathrm{m}$. As already mentioned in Sec. 2.3.1 the problem of solving this equation lies in the additional three dimensions for the velocity space.

The main idea of LB is to discretize the velocity space with only a small number of discrete velocities in a way that preserves certain conservation laws and thus models the macroscopic behavior of the fluid exactly ${ }^{6}$. This is possible when recalling that the moments of the distribution function, i.e., the macroscopic quantities of interest, are written as integrals of $f$ and $\boldsymbol{v}$. Specifically, in the case of fluid dynamics we write the zeroth and first moment, i.e., the density and fluid velocity as

$$
\begin{aligned}
\rho(\boldsymbol{r}, t) & :=m \int f(\boldsymbol{r}, \boldsymbol{v}, t) \mathrm{d}^{(3)} \boldsymbol{v}, \\
\boldsymbol{u}(\boldsymbol{r}, t) & :=\frac{m}{\rho} \int f(\boldsymbol{r}, \boldsymbol{v}, t) \boldsymbol{v} \mathrm{d}^{(3)} \boldsymbol{v} .
\end{aligned}
$$

Using the Gauss-Hermite quadrature rule allows the exact numerical evaluation of these integrals in terms of a discrete sum, i.e.,

$$
\rho=\sum_{i}^{N_{\text {pop }}} f_{(i)}, \quad \boldsymbol{u}=\frac{1}{\rho} \sum_{i}^{N_{\text {pop }}} f_{(i)} \boldsymbol{v}_{(i)},
$$

where an appropriate scaling has been used to make the quantities dimensionless. Note the equal signs "=" instead of " $\approx$ " meaning that these discrete sums evaluate to the exact same moments as the continuous integrals do. This requires a suitable discretization of $f$ in terms of $N_{\text {pop }}$ populations $f_{(i)}\left(\boldsymbol{r}, \boldsymbol{v}_{(i)}, t\right)$. For simplicity we will write the discrete populations of the distribution function simply as $f_{(i)}$, where the subscript $(i)$ is not a tensor index, but denotes the $i$ th population. Each population is the distribution of particles in time and space that propagate only in direction $\boldsymbol{v}_{(i)}$. While the exact microscopic behavior is lost due to this discretization, it maintains the correct macroscopic behavior.

The heart of an LB scheme is now to find the correct discrete velocities $\boldsymbol{v}_{(i)}$ and the corresponding weights $w_{i}$ that connect each population to the continuous distribution function via $f_{(i)} \propto w_{i} f$. Since this discretization is derived from the exact solution of specific integrals, it is referred to as quadrature rule or simply quadrature. While other approaches are possible (He \& Luo, 1997), the use of Hermite polynomials (Shan \& He, 1998) proves particularly useful for deriving this quadrature. This is due to the fact that an expansion of the distribution function in terms of orthogonal Hermite polynomials, $H^{(k)}$, results in expansion coefficients, $a^{(k)}$, that coincide with the moments (Grad, 1949b,a). To be more precise, the quadrature is derived from the expansion of the equilibrium distribution function, $f$ eq, which in contrast to $f$ is a known function. Using the Maxwell-Boltzmann distribution as equilibrium for a classical fluid as an example, the expansion in $d$ dimensions reads

$$
f^{\mathrm{eq}} \approx(2 \pi)^{-d / 2} \exp \left(\frac{\boldsymbol{v}^{2}}{2}\right) \sum_{k}^{S} a^{(k)}(\rho, \boldsymbol{u}) H^{(k)}(\boldsymbol{v}),
$$

which is truncated at order $S$. Formally, $S$ is the highest order moment that one wants to recover exactly, which determines the number of required populations as

\footnotetext{
${ }^{6}$ This means that LB is not actually solving the Boltzmann equation. Rather, it should be considered as a numerical solution of the macroscopic Navier-Stokes equation. This is due to the fact that LB - as will be explained below - loses the microscopic information and only retains that of a few macroscopic moments.
} 
$N_{\text {pop }} \geq(S+1) / 2$ (Krüger et al., 2017).

Conservation of particle number, energy, and momentum allow to write the moments in terms of $f^{\text {eq }}$ as well as $f$, i.e.,

$$
\int f \boldsymbol{v}^{S} \mathrm{~d}^{d} v=\int f^{\mathrm{eq}} \boldsymbol{v}^{S} \mathrm{~d}^{d} \boldsymbol{v}
$$

This allows to use the same discretization that can be derived for the know equilibrium distribution also for the unknown distribution $f$. It is already noted at this point, that this is longer true in the case of radiation. The fundamental difference is that in fluid mechanics the particles interact with themselves and thus conserve particle number, energy, and momentum. This is no longer true for radiation, which instead interacts with the underlying fluid. In this case particles are destroyed and created and energy and momentum is exchanged with the fluid. Consequently, conservation laws no longer apply when considering radiation alone (of course energy and momentum is conserved over the combined system of fluid and radiation) and thus the derivation of an exact quadrature as done for classical LB is no longer possible.

Having found a suitable discretization for the velocity space then results in the LB equation

$$
f_{(i)}\left(\boldsymbol{r}+\boldsymbol{v}_{(i)} \Delta t, t+\Delta t\right)=f_{(i)}(\boldsymbol{r}, t)+\Delta \mathcal{C}_{(i)},
$$

where a simple first-order discretization for the time is used. Typically, LB uses a grid with a grid spacing of $\Delta x=\Delta t=1$, which can be arbitrarily scaled.

The collisional operator $\mathcal{C}$ is most commonly written in the form of the BGK relaxation time approximation (Bhatnagar et al., 1954)

$$
\mathcal{C}_{(i)}=\frac{1}{\tau}\left(f_{(i)}-f_{(i)}^{\mathrm{eq}}\right),
$$

where $\tau$ is the system's relaxation time. This form highlights the fact that the distribution function tends to relax towards an equilibrium, i.e., $f^{\text {eq }}$.

Eq. (5.93) is solved via the stream-and-collide approach, which splits the solution procedure in two parts. First the particle collisions are modeled via the collisional source term as

$$
f_{(i)}^{*}=f_{(i)}+\Delta t \mathcal{C}_{(i)},
$$

resulting in an intermediate distribution. In the second step the particles stream along the discrete velocity directions, $\boldsymbol{v}_{(i)}$, to their neighboring cells. Numerically, this is evaluated as

$$
f_{(i)}\left(\boldsymbol{r}+\boldsymbol{v}_{(i)} \Delta t, t+\Delta t\right)=f_{(i)}^{*}(\boldsymbol{r}, t) .
$$

Fig. 5.14 shows this graphically and clarifies that the distribution $f_{(i)}^{*}$ at time $t$ and position $\boldsymbol{r}$ will become the new distribution $f_{(i)}$ at time $t+\Delta t$ and position $\boldsymbol{r}+\boldsymbol{v}_{(i)} \Delta t$.

This stream-and-collide approach will also be adopted for the radiative LB method. It needs to be modified, however, because radiation always streams at the speed of light in all directions, i.e., all discrete velocities need to have the same magnitude. This leads to populations streaming off-grid, i.e., not terminating at a neighboring cell-center.

The described classical LB method is suitable for hydrodynamical systems, where the mean free path of particles is much shorter than the typical length scale of the system itself. The ratio of these two lengths is the Knudsen number. For small Knudsen number LB has routinely been used for several decades (e.g., in Massaioli et al., 1993; He et al., 1998; Peng et al., 2003; Karlin et al., 2013). For a large 


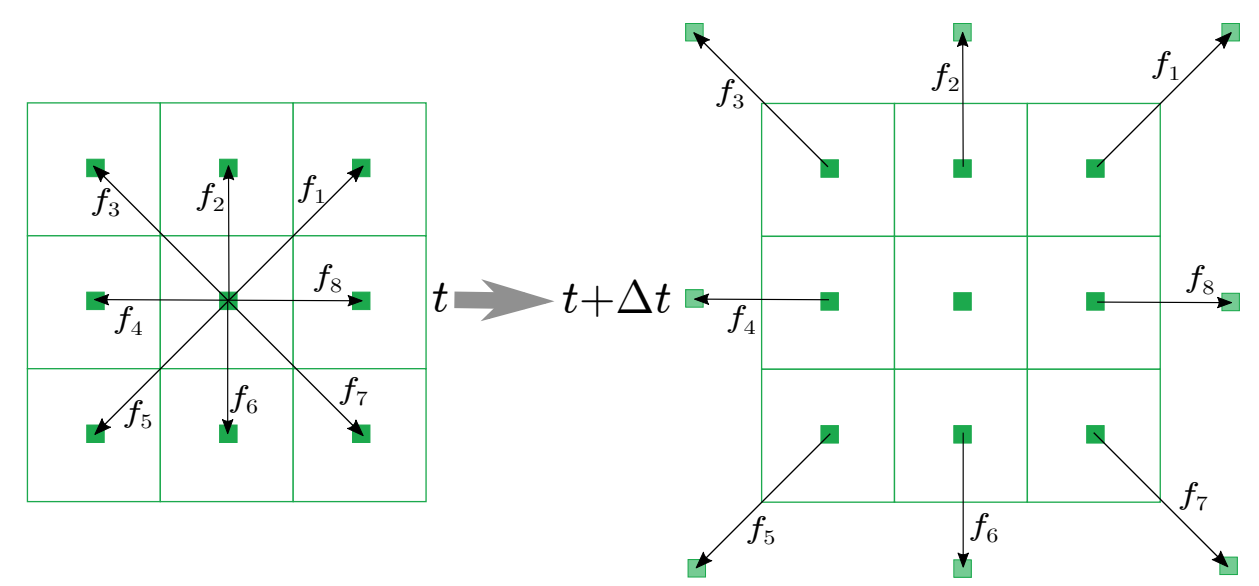

Figure 5.14: Visual representation of the streaming step in the LB method. The figure shows the streaming of eight populations from the central cell to the surrounding neighbors. Note that each of the neighbors also receives populations from other surrounding grid cells, which is not shown here. Likewise the central cell receives eight populations, one from each of its surrounding neighbors (also not shown).

Knudsen number, on the other hand, the relaxation time approximation is no longer applicable, which will become a problem for the free-streaming regime for radiation particles. Nevertheless, pioneering studies by Asinari et al. (2010); Bindra \& Patil (2012); Mishra et al. (2014); McCulloch \& Bindra (2016); Yi et al. (2016); Vernekar \& Mishra (2014) have applied LB in one and two dimensions also to radiative transport. These studies were limited by only computing steady-state solutions as a consequence of the off-grid streaming for radiation particles ${ }^{7}$. McHardy et al. (2016); Mink et al. (2020) have even used LB for three-dimensional radiative transport.

None of these schemes is suitable for radiative transport in high-energy astrophysics, where a) the time evolution of the system has to be physically realistic and b) not only the diffusion regime, but also the free-streaming regime (high Knudsen number) needs to be modeled correctly. Further details, like the inclusion of anisotropic scattering, backreaction with the underlying fluid, motion of the fluid, stiff source terms, etc. also need to be taken into account. Based on Weih et al. (2020a) we present an extension of the LB method that is suitable for radiative transport in high-energy astrophysics. Note that the scheme is by no means complete and only represents a first step in the right direction. Specifically, we ignore curved spacetime and only treat the special-relativistic case. We also restrict the derivation to the gray approximation. In principle, both general relativity and frequency-dependence can be included in the new scheme. Here, however, we only work out the basics and provide a proof-of-concept for the applicability of LB to numerical astrophysics.

\footnotetext{
7 A simple way to solve this problem is to allow for different streaming velocities, so that all populations always stream to neighboring cell centers. This is unphysical and so is the evolution of the system. The final equilibrium, however, is not impacted by this unphysical evolution.
} 


\subsubsection{Derivation of the Lattice Boltzmann Method for Ra- diative Transport}

\section{The Underlying Master Equation}

For convenience we here repeat the RTE (see Sec. 2.3.1), i.e., the Boltzmann equation for massless particles with the source term given in Eq. (2.79):

$$
\left(\frac{\partial}{\partial t}+\hat{\boldsymbol{n}} \cdot \boldsymbol{\nabla}\right) I_{(\nu)}=\eta_{(\nu)}-\kappa_{a,(\nu)} I_{(\nu)}+\nu^{3} \mathcal{C}_{\text {scat }} .
$$

In contrast to Eq. (2.82), we use vector notation, i.e., writing $\hat{\boldsymbol{n}}$ instead of individual components $\hat{n}^{i}$. We recall that $\hat{\boldsymbol{n}}$ is a unit vector in the direction of propagation of the radiation particle and $I_{(\nu)}$ is the frequency-dependent specific radiation intensity defined in Eq. (2.81). We also recall that the first, second, and third term on the RHS are for emission, absorption, and scattering, respectively.

It is now time to write an explicit expression for $\mathcal{C}_{\text {scat }}$ that can be implemented. For this we start from the generic form given by Eq. (2.80). The scattering kernels, $R^{\text {in }}\left(\nu, \nu^{\prime}\right)$ and $R^{\text {out }}\left(\nu, \nu^{\prime}\right)$ depend on the underlying physical process and in general do not allow for an analytic solution of the integrals in Eq. (2.80). A commonly used approximation is a truncated Legendre expansion for these kernels (Bruenn, 1985; Rampp, 2000; Shibata et al., 2011), i.e.,

$$
R^{\text {in } / \text { out }}\left(\nu, \nu^{\prime}\right) \approx \frac{1}{2} \Phi_{0}^{\text {in/out }}\left(\nu, \nu^{\prime}\right)+\frac{3}{2} \Phi_{1}^{\text {in } / \text { out }} \cos \theta .
$$

Here, $\theta$ is the angle between ingoing and outgoing radiation, so that $\cos \theta=\hat{\boldsymbol{n}} \cdot \hat{\boldsymbol{n}}^{\prime}$, and $\Phi_{\ell=0,1}^{\text {in } / \text { out }}$ are the first two coefficients of the Legendre expansion. Note that the second term also allows for non-isotropic scattering. A further simplification is the restriction to iso-energetic scattering, i.e., to only scattering processes during which the scattered particle's energy does not change. Consequently

$$
\Phi_{\ell}^{\text {in }}\left(\nu, \nu^{\prime}\right)=\Phi_{\ell}^{\text {out }}\left(\nu, \nu^{\prime}\right)=: \Phi_{\ell}(\nu) \delta\left(\nu-\nu^{\prime}\right) .
$$

We can now evaluate Eq. (2.80) by inserting Eqs. (5.99) and (5.98). We then obtain an expression that is written in terms of the moments as

$$
\mathcal{C}_{\text {scat }} \approx-\kappa_{0,(\nu)} f_{(\nu)}+\kappa_{0,(\nu)} E_{(\nu)}+3 \kappa_{1,(\nu)} \hat{\boldsymbol{n}} \cdot \boldsymbol{F}_{(\nu)},
$$

where $\kappa_{\ell,(\nu)}:=2 \pi \nu^{2} \Phi_{\ell}$ (Rampp, 2000) and we recall the definition of the two lowest moments

$$
E_{(\nu)}:=\frac{1}{4 \pi} \int_{4 \pi} f_{(\nu)} \mathrm{d} \Omega \quad \text { and } \quad \boldsymbol{F}_{(\nu)}:=\frac{1}{4 \pi} \int_{4 \pi} \hat{\boldsymbol{n}} f_{(\nu)} \mathrm{d} \Omega .
$$

Using the gray approximation, i.e., integrating Eq. (5.97) over frequency space yields

$$
\left(\frac{\partial}{\partial t}+\hat{\boldsymbol{n}} \boldsymbol{\nabla}\right) I=-\kappa_{a} I+\eta+\kappa_{0}(E-I)+3 \kappa_{1} \hat{\boldsymbol{n}} \cdot \boldsymbol{F},
$$

where we use the analogous definition for the frequency-averaged opacities and frequencyintegrated emissivity as in Eqs. (2.92) and (2.93). This is the final master equation that we will discretize within our LB scheme. 


\section{LB Discretization of the RTE}

Before discretizing Eq. (5.102) we rewrite it in a form closer to that of an LB scheme. Specifically we write the RHS in BGK-form with an additional source term, i.e.,

$$
\left(\frac{\partial}{\partial t}+\hat{\boldsymbol{n}} \boldsymbol{\nabla}\right) I=-\kappa_{0}\left(I-I^{\mathrm{eq}}\right)+S
$$

where $S:=-\kappa_{a} I+\eta$ and the scattering term has been rearranged in order to introduce the "equilibrium" of the radiation intensity

$$
I^{\mathrm{eq}}:=E+\lambda \hat{\boldsymbol{n}} \cdot \boldsymbol{F} \quad \text { with } \quad \lambda:=3 \frac{\kappa_{1}}{\kappa_{0}} .
$$

Next, we assume a discretization for $\hat{\boldsymbol{n}}$ in terms of $N_{\text {pop }}$ directions $\hat{\boldsymbol{n}}_{(i)}$. It will be detailed below how to choose this discretization. What should be noted already here is that a quadrature that results in an exact recovery of the moments - as for the classical LB method - is no longer possible due to the lack of conservation laws. This also means that the moments can only be computed approximately from the discrete intensities, i.e.,

$$
E \approx \sum_{i}^{N_{\text {pop }}} I_{(i)}, \quad \boldsymbol{F} \approx \sum_{i}^{N_{\text {pop }}} \hat{\boldsymbol{n}}_{(i)} I_{(i)} .
$$

Likewise, the higher gray moments can be computed as

$$
M^{j_{1} \ldots j_{m}} \approx \sum_{i}^{N_{\mathrm{pop}}} n_{(i)}^{j_{1}} \ldots n_{(i)}^{j_{m}} I_{(i)} .
$$

The populations $I_{(i)}$ are the $N_{\text {pop }}$ discrete intensities that result from the discretization of the velocity space. Consequently, also Eq. (5.102) turns into $N_{\text {pop }}$ equations

$$
\left(\frac{\partial}{\partial t}+\hat{\boldsymbol{n}}_{(i)} \cdot \boldsymbol{\nabla}\right) I_{(i)}=-\kappa_{0}\left(I_{(i)}-I_{(i)}^{\mathrm{eq}}\right)+S_{(i)} .
$$

As is the case for classical LB, the weights, $w_{(i)}$, associated to $\hat{\boldsymbol{n}}_{(i)}$ enter via the equilibrium and the external source as

$$
I_{(i)}^{\mathrm{eq}}:=w_{(i)}\left(E+\lambda \hat{\boldsymbol{n}}_{(i)} \cdot \boldsymbol{F}\right) \quad \text { and } \quad S_{(i)}:=w_{(i)} \eta-\kappa_{a} I_{(i)} .
$$

Finally, a simple first-order discretization in time yields the new radiative Lattice Boltzmann equation

$$
I_{(i)}\left(\boldsymbol{r}+\hat{\boldsymbol{n}}_{(i)} \Delta t, t+\Delta t\right)=I_{(i)}(\boldsymbol{r}, t)-\kappa_{0} \Delta t\left(I_{(i)}(\boldsymbol{r}, t)-I_{(i)}^{\mathrm{eq}}(\boldsymbol{r}, t)\right)+\Delta t S_{(i)}(\boldsymbol{r}, t),
$$

which can be solved via the same stream-and-collide approach as the classical LB equation (see below for details on the numerical procedure).

While this completes the discretization and Eq. (5.109) can be implemented for numerical simulations, the missing ingredient is the recipe for discretizing the velocity space. As explained in Sec. 5.2.1 one of the most common approaches for classical LB methods is based on the Gauss-Hermite quadrature (Shan et al., 2006; Philippi et al., 2006; Shan, 2016). It has also been mentioned already, that this approach is no longer possible for radiation, because considering radiation particles (neutrinos or photons) alone, there are no conservation laws that allow the derivation of a suitable quadrature. Fortunately, there are other possibilities like the construction of a 
suitable velocity set as $d$-dimensional projection of known $(d+1)$-dimensional velocity sets stemming from the early days of LB (D'Humières et al., 1986). The most promising approach for radiative transfer, however, consists of defining a number of conditions that need to be fulfilled by the velocity set. Specifically, we will define a number of symmetry conditions (isotropy) that apply to the different kinematic regimes encountered in numerical astrophysics, from which we can derive a suitable discretization of the velocity space.

A first condition that needs to be satisfied by the desired velocity discretization is n-th order isotropy. Mathematically, this means (Rivet \& Boon, 2001)

$$
T^{\alpha_{1} \ldots \alpha_{k}}=\left\{\begin{array}{ll}
=0 & \text { for } k \text { odd }, \\
\propto \sum_{\text {perm }}\left(\delta_{\alpha_{1} \alpha_{2}} \ldots \delta_{\alpha_{k-1} \alpha_{k}}\right) & \text { for } k \text { even }
\end{array},\right.
$$

where we define the tensor of rank $\mathrm{k}$ as

$$
T^{\alpha_{1} \ldots \alpha_{k}}:=\sum_{i}^{N_{\mathrm{pop}}} w_{(i)} n_{(i)}^{\alpha_{1}} \ldots n_{(i)}^{\alpha_{k}} .
$$

Another condition that the velocity set needs to satisfy follows from the fact that massless particles always travel at the constant speed of light. This information is already present in the fact that $\hat{\boldsymbol{n}}_{(i)}$ are unit vectors (they all have the same magnitude) and hence span the surface of a circle in two dimension and that of a sphere in three dimensions. Such a spherical velocity set is used in classical LB for simulations of ultra-relativistic hydrodynamics. Two possibilities exist for realizing this scenario. For the first one, a velocity set is defined so that each $\hat{\boldsymbol{n}}_{(i)}$ is an integer multiple of the constant propagation speed ( $c$ in this case) and ends on a cell center of the Cartesian spatial grid (see e.g., Mendoza et al., 2013; Gabbana et al., 2018). This preserves the exact-streaming property, which is also visualized in Fig. 5.14. The second possibility allows for discrete velocities that stream off-grid, i.e., do not end in cell centers (see Fig 5.15). In classical LB this is used when going beyond hydrodynamics and whenever the usage of on-lattice velocity sets would require impractically large grids (Coelho et al., 2018; Ambrus \& Blaga, 2018). We opt for the second option since the treatment of the free-streaming regime, which is present in many astrophysical simulations, requires a large number of discrete velocities anyway. This will come at the cost of requiring an interpolation for the off-lattice streaming (see also below). This also makes sense when thinking of the eventual extension to general relativity, where streaming in a curved spacetime would also require interpolation.

Finally, we also desire to reproduce the moments from the discrete sums as accurate as possible. In practice, this means that we need to reproduce spherical integrals by discrete sums, i.e.,

$$
Q(h):=\frac{1}{4 \pi} \int_{4 \pi} h(\hat{\boldsymbol{n}}) \mathrm{d} \Omega \approx \sum_{i}^{N_{\mathrm{pop}}} w_{(i)} h\left(\hat{\boldsymbol{n}}_{(i)}\right),
$$

where $h(\hat{\boldsymbol{n}})$ is a generic square-integrable function of $\hat{\boldsymbol{n}}$. Such a function can be represented in terms of a series of orthogonal spherical harmonics, $Y_{\ell}^{m}$, on the unit sphere as (Atkinson \& Han, 2012)

$$
h(\hat{\boldsymbol{n}})=h(\theta, \phi)=\sum_{\ell=0}^{\infty} \sum_{m=-\ell}^{\ell} c_{\ell m} Y_{\ell}^{m}(\theta, \phi),
$$




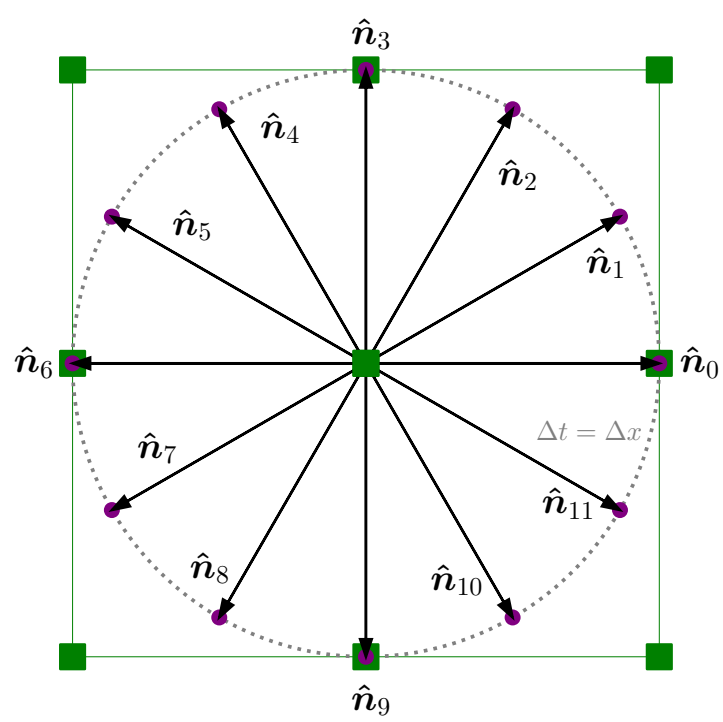

FIgURE 5.15: Example of a two-dimensional velocity stencil for the radiative LB method with twelve populations. The green squares represent cell centers, while the arrows show the discrete velocities, $\hat{\boldsymbol{n}}_{(i)}$, that are constructed according to the three conditions discussed in the main text. All velocities end on the unit circle (dotted) with radius $c \Delta t$, where $\Delta t$ is assumed to be in units of the grid-spacing. Reprint of Fig. 1 in Weih et al. (2020a).

where the coefficients $c_{\ell m}$ determine the convergence rate of the expansion and the angles $\theta$ and $\phi$ determine the velocity direction. From this we can determine the weights $w_{(i)}$ and the discrete velocities $\hat{\boldsymbol{n}}_{(i)}=\left(\theta_{(i)}, \phi_{(i)}\right)$ via the spherical quadrature rule, which is of order $p$ if it integrates all spherical harmonics $Y_{\ell}^{m}(\theta, \phi)$ up to order $\ell=p$ exactly, i.e.,

$$
\frac{1}{4 \pi} \int_{4 \pi} Y_{\ell}^{m}(\theta, \phi) \mathrm{d} \Omega=\sum_{k}^{N_{\text {pop }}} w_{(k)} Y_{\ell}^{m}\left(\theta_{(k)}, \phi_{(k)}\right), \quad \forall \ell \leq p .
$$

From this follows that all functions $Q(h)$ that correspond to integrals of functions $h(\hat{\boldsymbol{n}})$ that include products of $\hat{\boldsymbol{n}}$ up to order $p$ can be computed exactly by a discrete sum of the first $p$ harmonics. On the other hand, integrals over functions $h(\hat{\boldsymbol{n}})$ that include higher orders are only approximated by the sum of these $p$ harmonics. At the same time it can be shown that quadratures of order $p$ satisfy Eq. 5.110, which we stated as first condition for ensuring isotropy in the diffusion regime, up to order $n=p$. In other words, the quadrature order $p$ also determines the order of isotropy, which is why consequently these two terms will be used interchangeably.

While the above specification of the conditions that need to be satisfied by the velocity set seems rather complicated, it becomes much clearer when first considering the specific case of two dimensions. In two dimensions the integral in Eq. (5.112) has to be computed only on the unit circle. In this case the expansion of $h(\hat{\boldsymbol{n}})$ in Eq. (5.113) reduces to a simple Fourier series of the form

$$
h(\hat{\boldsymbol{n}})=h(\phi)=\sum_{m=\infty}^{+\infty} c_{m} e^{i m \phi},
$$


The final relation for determining the quadrature, i.e., Eq. (5.114) reduces to

$$
\frac{1}{2 \pi} \int_{0}^{2 \pi} e^{i m \phi} \mathrm{d} \phi=\sum_{k=0}^{N_{\text {pop }}-1} w_{(k)} e^{i m \phi_{(k)}} \quad \forall|m| \leq p .
$$

This relation can easily be satisfied by choosing $N_{\text {pop }}=p+1$ many vectors that are evenly distributed on the unit circle. This corresponds to $\hat{\boldsymbol{n}}_{(i)}=\left[\cos \phi_{(i)}, \sin \phi_{(i)}\right]^{T}$ with $\phi_{(i)}=2 \pi i / N_{\text {pop }}$ with equal weights $w_{(i)}=1 / N_{\text {pop }}$. A visual representation of this velocity set is shown in Fig. 5.15 for $N_{\text {pop }}=12$. Looking at this picture makes clear why in LB terminology a velocity set is also called stencil.

The derivation of such a stencil in three dimensions is far less trivial. Indeed, the definition of such a quadrature rule on a sphere is still an unsolved problem. Extensive research on this topic reveals that there are various approaches with different advantages and disadvantages (Beentjes, 2015; Gamba et al., 2017; Gross \& Atzberger, 2018; Lutsko \& Lam, 2018; Stepán, Jirí et al., 2020). For our purpose we borrow three of these spherical quadrature schemes, namely the

1.) Gauss-Legendre quadrature,

2.) Lebedev quadrature, and

3.) spherical-design quadrature.

The most straight-forward approach is that of the Gauss-Legendre quadrature. Making use of the separability of spherical harmonics into circular functions and Legendre polynomials, $P_{\ell}^{m}(\cos \theta)$, this quadrature is a trivial extension of the twodimensional case. The integral in Eq. (5.114) is then split as

$$
\int_{4 \pi} Y_{\ell}^{m}(\theta, \phi) \mathrm{d} \Omega \propto\left(\int_{0}^{\pi} P_{\ell}^{m}(\cos \theta) \sin \theta \mathrm{d} \theta\right)\left(\int_{0}^{2 \pi} e^{i m \phi} \mathrm{d} \phi\right) .
$$

Separating the two-dimensional integrals into these one-dimensional integrals allows to evaluate each with a one-dimensional quadrature. The $\theta$-integral can be evaluated via a one-dimensional Gauss-Legendre quadrature (Hildebrand, 1956) and the $\phi$-integral via the trapezoidal rule. An example of the resulting stencil is shown in the left panel of Fig. 5.16. Obviously, such a quadrature leads to an accumulation of points at the sphere's poles. Intuitively, one can think of this stencil as an extension of the two-dimensional one (Fig. 5.15) via rotating it by 180 degrees in $\phi$ direction. This naive discretization is also what is typically used in full Boltzmann solvers (e.g., in Iwakami et al., 2020).

A more isotropic discretization is achieved by the Lebedev quadrature. This more complex approach solves the integral in Eq. (5.114) as a system of non-linear equations of $w_{(i)}, \theta_{(i)}$, and $\phi_{(i)}$ instead of solving the product of two one-dimensional integrals. This system of equation is then solved by considering only the spherical harmonics that are invariant under transformation of a specific group $G$ (Sobolev, 1962). The resulting quadrature is then also invariant under these transformation, which means that for all $Q(h)$ that are computed exactly by the quadrature also $Q(g(h))$ is exact for all $g \in G$. Originally, Lebedev used the octahedral group for the derivation (Lebedev, 1975, 1976, 1977), but also other groups have been used since then (see e.g., Ahrens \& Beylkin, 2009). An example of a stencil derived from the Lebedev quadrature rule is shown in the middle panel of Fig. 5.16. Evidently, the discrete velocities are distributed much more uniformly when comparing to the Gauss-Legendre stencil. 


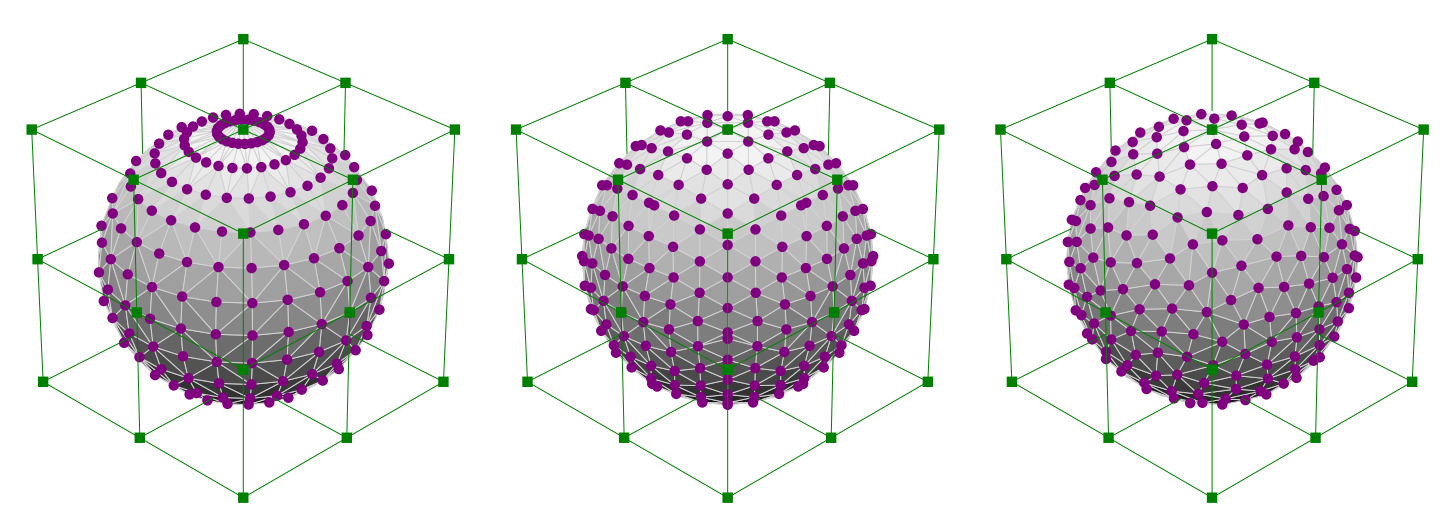

Figure 5.16: Example for the stencils derived from different types of quadrature. From left to right we compare Gauss-Legendre, Lebedev, and spherical-design stencils. As in Fig. 5.15 the green squares show the cell centers of the three-dimensional grid and the endpoints of the discrete velocities (purple) end on a sphere of radius $c \Delta t$. Reprint of Fig. 2 in Weih et al. (2020a).

Finally, we also consider the spherical-design quadrature proposed by Delsarte et al. (1977). In contrast to the previous two approaches, the spherical-design quadrature additionally requires all weights to be equal, which should in principle result in a more isotropic numerical evolution of the radiation. One then has to find a minimal set of points on the unit sphere that yields $Q(h)$ exactly up to order $p$. So far this can only be done numerically and we here use numerical results by Womersley (2018). An example of one of these stencils is shown in the right panel of Fig. 5.16. Note that while the Lebedev stencil still shows some points very close to each other (e.g., just slightly below the center), this is not the case for the spherical-design stencil.

It is not possible to identify beforehand which of these stencils will give the best results for our radiative LB method. We therefore test all of them when implementing the new method (see Sec. 5.2.3).

\section{Numerical Procedure}

We now have all the ingredients to summarize the implementation for solving Eq. (5.109) numerically. As already mentioned we use the stream-and-collide approach from the classical LB method.

We start with the streaming step, from which we obtain an intermediate value for the updated intensities, i.e.,

$$
I_{(i)}^{*}(\boldsymbol{r}, t+\Delta t)=I_{(i)}\left(\boldsymbol{r}-\hat{\boldsymbol{n}}_{(i)} \Delta t, t\right)
$$

In general, the discrete velocities will be off-grid, so that there is no value for $I_{(i)}$ at location $\left(\boldsymbol{r}-\hat{\boldsymbol{n}}_{(i)} \Delta t\right)$. We thus use an interpolation for providing this value. A simple trilinear interpolation scheme then yields

$$
\begin{aligned}
& I_{(i)}^{*}(\boldsymbol{r}, t+\Delta t)=I_{(i)}\left(\boldsymbol{r}-\hat{\boldsymbol{n}}_{(i)} \Delta t, t\right) \approx \frac{1}{\Delta x \Delta y \Delta z} \times\{ \\
& I_{(i)}(\boldsymbol{r}-\hat{\boldsymbol{x}}-\hat{\boldsymbol{y}}-\hat{\boldsymbol{z}}, t)\left(\Delta t\left|\hat{n}_{(i)}^{x}\right|\right)\left(\Delta t\left|\hat{n}_{(i)}^{y}\right|\right)\left(\Delta t\left|\hat{n}_{(i)}^{z}\right|\right)+ \\
& I_{(i)}(\boldsymbol{r}-\hat{\boldsymbol{y}}-\hat{\boldsymbol{z}}, t)\left(\Delta x-\Delta t\left|\hat{n}_{(i)}^{x}\right|\right)\left(\quad \Delta t\left|\hat{n}_{(i)}^{y}\right|\right)\left(\quad \Delta t\left|\hat{n}_{(i)}^{z}\right|\right)+ \\
& I_{(i)}(\boldsymbol{r}-\hat{\boldsymbol{x}} \quad-\hat{\boldsymbol{z}}, t)\left(\quad \Delta t\left|\hat{n}_{(i)}^{x}\right|\right)\left(\Delta y-\Delta t\left|\hat{n}_{(i)}^{y}\right|\right)\left(\quad \Delta t\left|\hat{n}_{(i)}^{z}\right|\right)+
\end{aligned}
$$




$$
\begin{aligned}
& I_{(i)}(\boldsymbol{r}-\hat{\boldsymbol{x}}-\hat{\boldsymbol{y}} \quad, t)\left(\quad \Delta t\left|\hat{n}_{(i)}^{x}\right|\right)\left(\quad \Delta t\left|\hat{n}_{(i)}^{y}\right|\right)\left(\Delta z-\Delta t\left|\hat{n}_{(i)}^{z}\right|\right)+ \\
& I_{(i)}(\boldsymbol{r} \quad-\hat{z}, t)\left(\Delta x-\Delta t\left|\hat{n}_{(i)}^{x}\right|\right)\left(\Delta y-\Delta t\left|\hat{n}_{(i)}^{y}\right|\right)\left(\quad \Delta t\left|\hat{n}_{(i)}^{z}\right|\right)+
\end{aligned}
$$

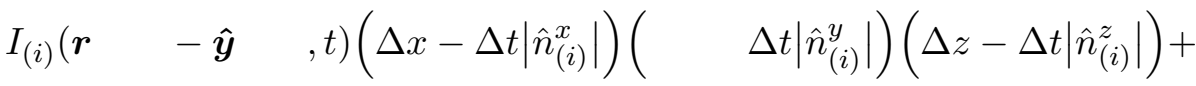

$$
\begin{aligned}
& I_{(i)}(\boldsymbol{r}-\hat{\boldsymbol{x}} \quad, t)\left(\quad \Delta t\left|\hat{n}_{(i)}^{x}\right|\right)\left(\Delta y-\Delta t\left|\hat{n}_{(i)}^{y}\right|\right)\left(\Delta z-\Delta t\left|\hat{n}_{(i)}^{z}\right|\right)+ \\
& \left.I_{(i)}(\boldsymbol{r} \quad, t)\left(\Delta x-\Delta t\left|\hat{n}_{(i)}^{x}\right|\right)\left(\Delta y-\Delta t\left|\hat{n}_{(i)}^{y}\right|\right)\left(\Delta z-\Delta t\left|\hat{n}_{(i)}^{z}\right|\right)\right\} \text {, }
\end{aligned}
$$

where

$\hat{\boldsymbol{x}}=\left(\operatorname{sign}\left(\hat{n}_{(i)}^{x}\right) \Delta x, 0,0\right)^{T}, \quad \hat{\boldsymbol{y}}=\left(0, \operatorname{sign}\left(\hat{n}_{(i)}^{y}\right) \Delta y, 0\right)^{T}, \quad \hat{\boldsymbol{z}}=\left(0,0, \operatorname{sign}\left(\hat{n}_{(i)}^{z}\right) \Delta z\right)^{T}$,

In this way the unknown intensities at $\left(\boldsymbol{r}-\hat{\boldsymbol{n}}_{(i)} \Delta t\right)$ can be approximated in terms of the known intensities at the surrounding cell-centers. The two-dimensional case follows equivalently from a bilinear interpolation.

Next, the collision step is performed, during which the intermediate $I_{(i)}^{*}$ is updated to the new value at time $t+\Delta t$. According to Eq. (5.109) we compute

$$
I_{(i)}(\boldsymbol{r}, t+\Delta t)=I_{(i)}^{*}(\boldsymbol{r}, t)-\kappa_{0} \Delta t\left(I_{(i)}^{*}(\boldsymbol{r}, t)-I_{(i)}^{\mathrm{eq}}(\boldsymbol{r}, t)\right)+\Delta t S_{(i)}(\boldsymbol{r}, t) .
$$

Note that according to Eq. (5.108) for the equilibrium intensity the macroscopic moments, $E$ and $\boldsymbol{F}$, need to be computed first. This can be done via Eq. (5.105).

In most astrophysical scenarios the opacities and emissivity can have numerical values that are much larger than the value of the evolved variables, $I_{(i)}$. This is the same problem as already discussed for the M1 method in Sec. 5.1 that leads to a stiff system of equations and consequently to an unstable evolution when using an explicit time stepper. An implicit treatment of the source terms is again the solution to this problem. Instead of Eq. (5.121) we then solve

$$
\begin{aligned}
I_{(i)}(\boldsymbol{r}, t+\Delta t) & =I_{(i)}^{*}(\boldsymbol{r}, t)-\kappa_{0} \Delta t\left(I_{(i)}^{*}(\boldsymbol{r}, t+\Delta t)-I_{(i)}^{\mathrm{eq}}(\boldsymbol{r}, t+\Delta t)\right) \\
& +\Delta t S_{(i)}(\boldsymbol{r}, t+\Delta t) .
\end{aligned}
$$

Since $I_{(i)}^{\text {eq }}$ depends via the moments on all populations $I_{(i)}$, we now have a system of $N_{\text {pop }}$ linear equations with unknowns $I_{(i)}$. In principle this could be solved, e.g., via the inversion of an $N_{\text {pop }} \times N_{\text {pop }}$ matrix at every grid point or any other numerical method for solving systems of linear equations. This, however, becomes unfeasible for large $N_{\text {pop }}$, especially in the three-dimensional case. At the latest when going to a coupled frequency-dependent scheme, which breaks the linearity of the system, such methods would no longer be usable. We therefore use a different approach known as Lambda iteration (Rampp, 2000). Within this method we do the following at every grid cell:

1.) Start with an initial guess for $E(\boldsymbol{r}, t+\Delta t)$ and $\boldsymbol{F}(\boldsymbol{r}, t+\Delta t)$. We simply use the moments at time $t$ as initial guess.

2.) Using these moments, compute $I_{(i)}^{\mathrm{eq}}(\boldsymbol{r}, t+\Delta t)$.

3.) With the known equilibrium intensities, Eq. (5.122) reduces to a set of $N_{\text {pop }}$ decoupled linear equations that can each be solved analytically to obtain a first estimate of $I_{(i)}(\boldsymbol{r}, t+\Delta t)$. 
4.) Use this estimate to obtain an improved guess for $E(\boldsymbol{r}, t+\Delta t)$ and $\boldsymbol{F}(\boldsymbol{r}, t+\Delta t)$.

5.) Cycle back to step 2 and repeat the procedure until all $I_{(i)}(\boldsymbol{r}, t+\Delta t)$ converge to a value within the desired accuracy.

When coupling the radiation with a fluid (where the fluid is handled by a standard relativistic hydrodynamics code like BHAC), the backreaction onto the fluid in form of the collisional source term can easily be computed from the radiation moments at every time step. Returning this source term to the fluid code allows it to complete the update of the fluid variables. At the beginning of the next time step the parameters $\kappa_{0}$, $\kappa_{1}, \kappa_{a}$, and $\eta$ can then be computed from the new fluid variables. Special attention has to be paid to the case of a fluid that is in motion, which will be discussed in Sec. 5.2.4 and Appendix B.

Finally, we note that while we here use a simple first-order time stepper, the method can be easily extended to higher orders with an IMEX scheme (Pareschi \& Russo, 2005) as done for the M1 method (see Sec. 5.1.1).

\subsubsection{Code Tests and Comparison to M1}

After implementing the LB scheme as described in the previous section, we now verify not only this implementation, but also the methods capabilities in general. All tests are performed having an application to numerical astrophysics in mind. Specifically, this means that we perform tests in the optically thick, intermediate, and thin regime. Furthermore, we compare to the M1 scheme and the results obtained with M1 for the same tests (see also Sec. 5.1.2).

\section{Beam Tests}

We begin with the same beam tests as for the M1 method in the beginning of Sec. 5.1.2. For this we assume a vacuum background by setting $\kappa_{0}=\kappa_{1}=\kappa_{a}=\eta=0$. While the grid is the same (two dimensions) as for M1, the initialization differs. Instead of initializing the moments, we now have to initialize the $N_{\text {pop }}$ populations. We do so according to the condition

$$
I_{(i)}=\left\{\begin{array}{ll}
1 & \text { for } i=0, \\
0 & \text { for } i \neq 0
\end{array},\right.
$$

where $I_{(0)}$ corresponds to the population streaming in direction $\hat{\boldsymbol{n}}_{(0)}=(1,0)$, i.e., parallel to the x-axis. For this we use a simple stencil as shown in Fig. 5.15 with $N_{\text {pop }}=8$.

The top left panel of Fig. 5.17 shows the beam after $t=0.7$ with $\Delta t=\Delta x=\Delta y$. This choice is the most common one in classical LB methods and as can be seen from the figure results in perfect streaming, i.e., there is no diffusion ahead of the beam. Looking at Eq. (5.119), one can see that such a timestep leads to

$$
I_{(i)}^{*}(\boldsymbol{r}, t+\Delta t)=\frac{\left|\hat{n}_{(i)}^{x}\right|}{\Delta x} \times I_{(i)}(\boldsymbol{r}-\boldsymbol{x}, t) .
$$

Considering that $\left|\hat{n}_{(i)}^{x}\right| / \Delta x=1$ for $i=0$, makes clear that during the simulation the only non-zero intensity is simply propagated from the left to its neighboring cell-center to the right.

The situation changes when using $\Delta t<\Delta x$. This is indeed a more common choice in finite-differencing and finite-volume codes encountered in astrophysics, where the 


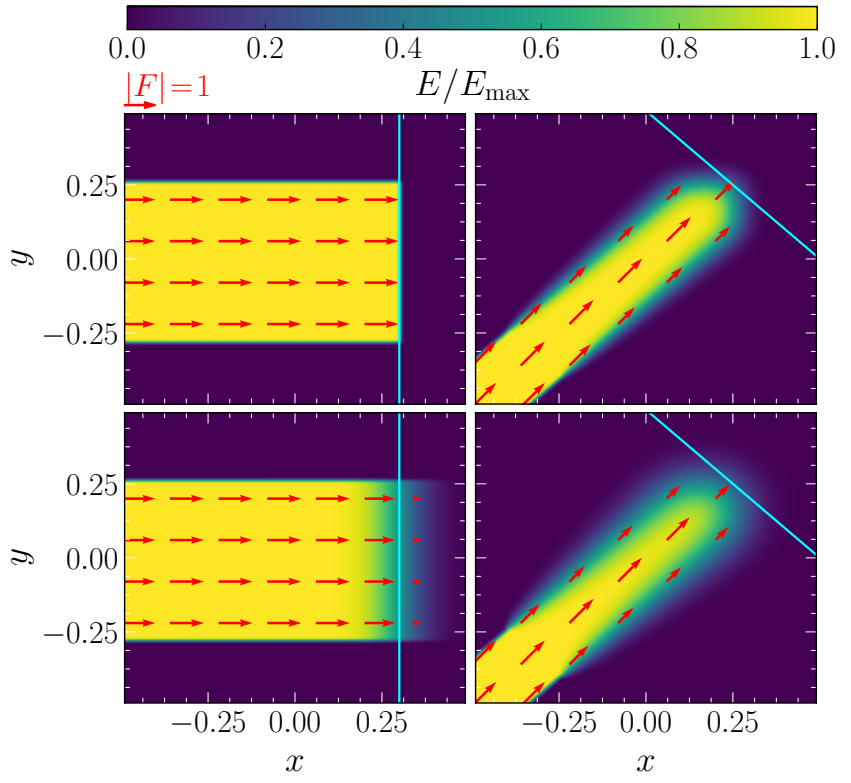

Figure 5.17: Left: Same propagating beam test as in Fig. 5.1 with a CFL-number of 1 (top) and 0.2 (bottom). Color coded is the radiation energy density, while red arrows show the momentum density. The cyan lines mark how far the beam should have traveled until $t=0.7$. Right: The same as the left panel, but for a diagonally propagating beam. Reprint of Fig. 3 in Weih et al. (2020a).

time step is limited by the CFL-condition (Rezzolla \& Zanotti, 2013, see also Sec. 2.2). Given that any realistic astrophysical simulation will require a coupling to such a code, we also perform the test for $\Delta t=0.2 \Delta x$. In the picture of Fig. 5.15 this corresponds to a squeezed stencil ${ }^{8}$. The result for this choice of $\Delta t$ is shown in the bottom left panel of Fig. 5.17. This time, one can see the same diffusion ahead of the beam as in the case of M1 (cf., Fig. 5.1). Due to the simple first-order time discretization the diffusion is slightly more than for M1.

We also perform the same test for a diagonal beam by initializing $I_{(1)}$ instead of $I_{0}$, where the former corresponds to the population streaming in direction $\hat{\boldsymbol{n}}=$ $[\cos (\pi / 4), \sin (\pi / 4)]$. The right panels of Fig. 5.17 show the results again for both choices of $\Delta t$ with the same results that could be observed previously. Note that the beam can only propagate in a direction that is provided by the stencil. This is indeed the biggest drawback of the LB method and will be further discussed in the next test.

Finally, we also analyze the case of two crossing beams. The M1 method is known to not correctly reproduce the behavior expected from such a scenario (Fragile et al., 2014; McKinney et al., 2014; Foucart et al., 2015; Rivera-Paleo \& Guzmán, 2019; Weih et al., 2020c); the beams will merge and result in a single beam in the average direction of the original beams, which is not what one would realistically expect from radiation ${ }^{9}$. We compare the results for LB with those obtained by FRAC in Fig. 5.18. Evidently, the LB method is able to reproduce the correct behavior of two crossing beams, while the M1 method is not. This is due to the fact, that M1 only evolves the two lowest

\footnotetext{
${ }^{8}$ Note that while this image of a squeezed stencil helps visualizing what happens, it is not what is actually implemented. The discrete velocities are always unit vectors, i.e., they are not affected by the choice of the time step.

${ }^{9}$ This is due to radiation not interacting with itself. While there are interactions between different neutrino species, in the case of neutrinos of the same flavor no reaction is expected so that - just like for photons - the beams should simply cross.
} 


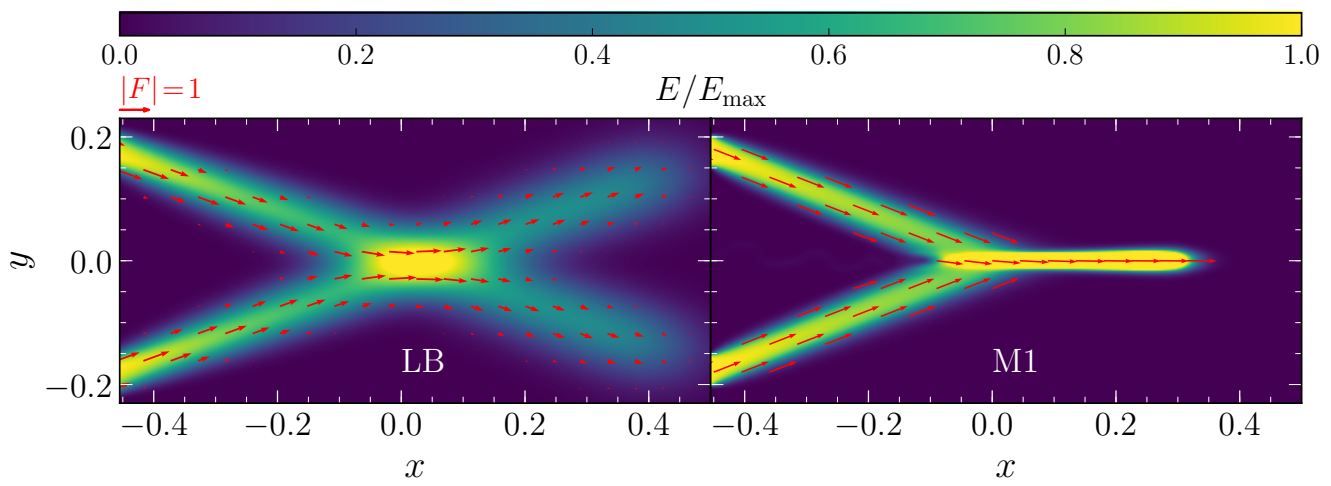

Figure 5.18: Comparison of the crossing-beam test between LB (left) and M1 (right). For LB the beams are initialized by setting the populations in the correct directions to a non-zero value. For M1 the momentum density is initialized to point towards the center. Reprint of Fig. 4 in Weih et al. (2020a).

moments, which allows for only one direction of propagation (radiation is basically treated like a fluid). LB, on the other hand, evolves the individual populations, which can cross without interacting.

\section{Radiation Wave in the Free-Streaming Regime}

Remaining in the free-streaming regime, we now test the expansion of a spherically symmetric radiation wave. This time, we use a three-dimensional grid in order to test the different types of stencil introduced in Sec. 5.2.2. The grid is made of $200^{3}$ uniformly sized grid-cells. We use $\Delta t=0.2$ and initialize all populations as $I_{(i)}=1$ within a sphere of radius $R=16 \Delta x$ and to zero everywhere else.

First, we show the simulation results when using a Lebedev stencil of order $p=23$ $\left(N_{\text {pop }}=194\right)$ in Fig. 5.19. As was the case for the M1 method, a ring-like structure can be seen propagating radially outwards (top panels). The same figure shows in the bottom panel, that the maximum radiation energy density falls off according to an inverse-square law.

Next, we perform the same test for all three types of quadrature introduced in Sec. 5.2.2. For each type we use three different quadrature orders. Note that the same order corresponds to different $N_{\text {pop }}$ for different types (the Gauss-Legendre quadrature, for example, requires more populations than the Lebedev quadrature for achieving the same order). The results for all $3 \times 3=9$ stencils are compared in Fig. 5.20 after 200 iterations. It can be seen that for all quadrature types a higher order gives smoother and more symmetric results. Specifically, we observe that $N_{\text {pop }} \sim 400$ is necessary in order to achieve spherical symmetry in the freestreaming regime. This is expected, when considering that the LB method is originally designed to work particularly well in the diffusion limit. In this sense free-streaming is the methods Achilles' heel. We will show below that the same test in the diffusion regime requires significantly less populations for satisfactory results. Nevertheless, $N_{\text {pop }} \sim 400$ is still a reasonable number of discrete velocities and - as will be shown requires a comparable computational effort as the M1 method does.

Between the different quadratures, we find no appreciable differences. From a mathematical point of view the Lebedev and spherical-design quadratures yield higher isotropy than the Gauss-Legendre quadrature. This difference, however, does not manifest in this simple test. On the other hand, the Lebedev quadrature yields 


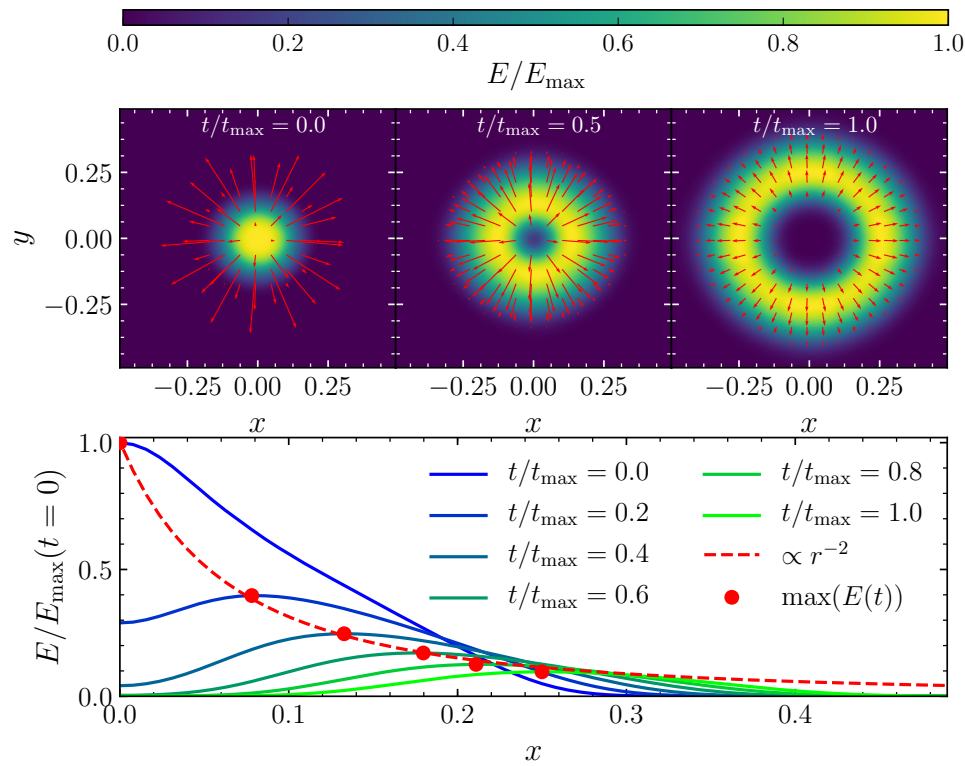

Figure 5.19: Freely propagating radiation wave. Top: Radiation energy (color-coded) and momentum density (red arrows) for three representative times. Bottom: Profiles of the radiation energy density at six different times. The red dots mark the maximum for each time. The red-dashed line is a fit of the form $\propto r^{-2}$ to these maxima. Reprint of Fig. 6 in Weih et al. (2020a).

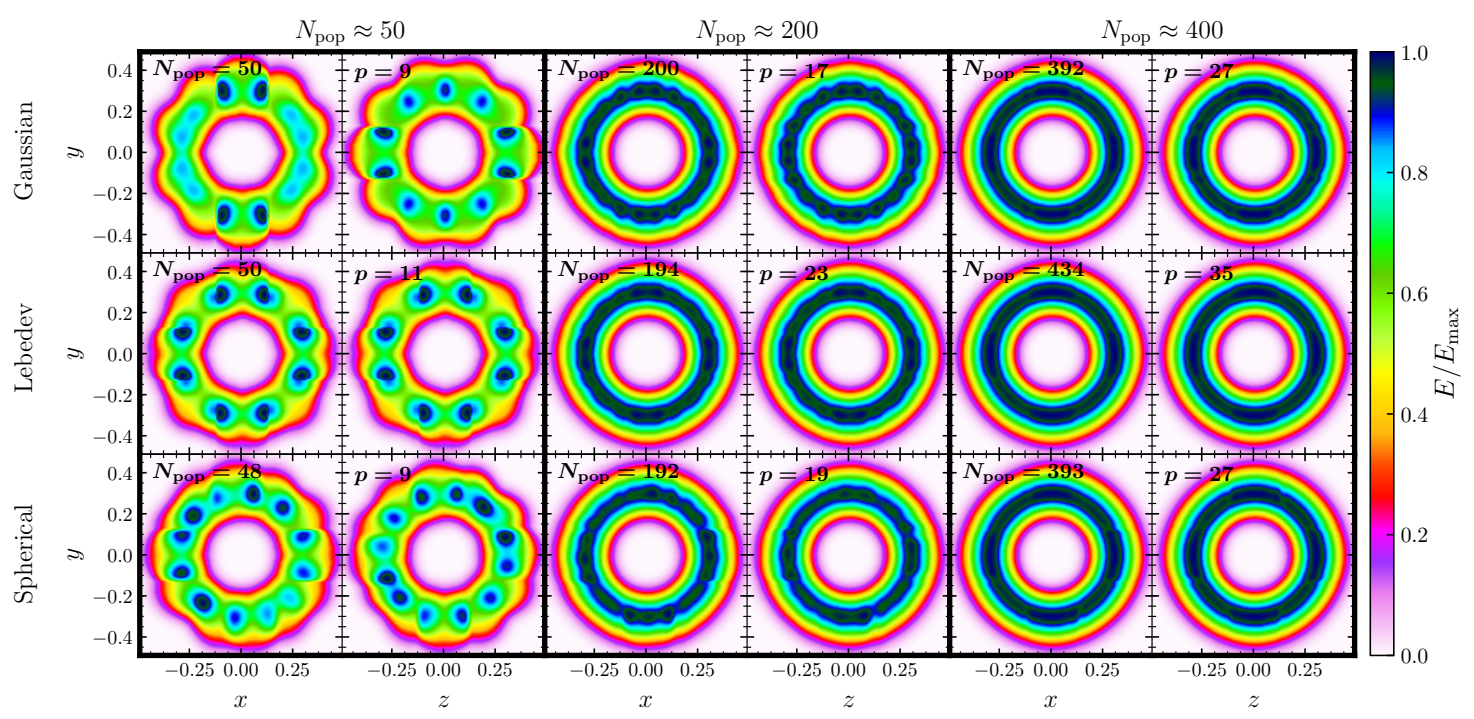

Figure 5.20: Freely-streaming radiation wave in the $(x, y)$ and $(x, z)$ plane for three different types of quadrature. From top to bottom we show results for the Gauss-Legendre, Lebedev, and spherical-design quadratures, respectively. For each type of quadrature stencils of different order are shown from left to right. Note that we always compare the (roughly) same $N_{\text {pop }}$, which means that we compare different quadrature orders $p$. Reprint of Fig. 5 in Weih et al. (2020a).

consistently the same symmetry as the other two quadratures, but for a smaller $N_{\text {pop }}$.

\section{Shadow Test}

The previous tests are all performed in vacuum. We now consider a fluid background 


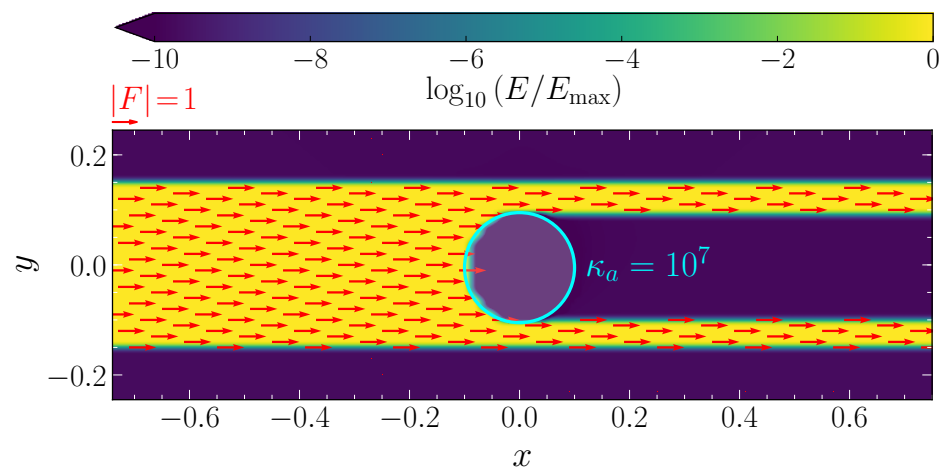

Figure 5.21: Shadow test for the LB method. The cyan colored circle shows the region within which the radiation is absorbed. Reprint of Fig. 7 in Weih et al. (2020a).

that absorbs radiation. Similar to the test of our implementation of the M1 method we simply set $\kappa_{a}=10^{7}$ within a sphere of radius $R=0.1$ at the origin of a grid of size $-0.75<x<0.75,-0.25<y, z<0.25$ covered by $150 \times 50 \times 50$ cells. The beam is initialized just like the straight beam in vacuum. For this we choose a stencil, which provides a velocity direction parallel to the x-axis. All other directions do not matter, because they will be set to zero.

Fig. 5.21 shows the results of this test. Evidently the absorbing material, marked by a cyan colored circle, absorbs the radiation and correctly produces a shadow as expected. Only a marginal amount of radiation diffuses inside the sphere. Note that the high absorption opacity causes the set of equations to become very stiff. The stable solution for a time step of $\Delta t=0.2 \Delta x$ is obtained thanks to the Lambda iteration method described at the end of Sec. 5.2.2. For this simple test we find the method to converge after at most three cycles within an accuracy of $\Delta E / E=10^{-14}$.

\section{Emitting Sphere Test}

Next, we repeat the emitting-sphere test (Smit et al., 1997), which we have already performed for the M1 method. We use a three-dimensional grid with $128^{3}$ uniformly sized cells. We choose a spherical-design stencil of order $20\left(N_{\text {pop }}=222\right)$. The test is performed for three different opacities, i.e., $\kappa_{a}=\eta=R^{-1}, 10 R^{-1}, 10^{10} R^{-1}$, where $R$ is the sphere's radius, which we choose to be an eighth of the total grid size.

The 222 populations' initialization only depends on the distance to the grid's center, $r$. We initialize

$$
I_{(i)}(r, t=0)=w_{(i)}\left\{\begin{array}{ll}
1 & \text { for } r<R \\
r^{-2} & \text { for } r \geq R
\end{array},\right.
$$

where we recall that $w_{(i)}$ are the weights associated to the stencil.

The results of these simulations are shown in terms of $E$ and $\boldsymbol{F}$ in Fig. 5.22. The left portion of the panels shows profiles for the LB method. As comparison we also show the results obtained for the same simulations with the M1 method in the right portions. For all three cases we find excellent agreement between the analytic solution [see Eqs. (5.63)-(5.66)] and the LB method. For the high-opacity case (blue) this is again thanks to the implicit time stepper. As already discussed in Sec. 5.1.2 the M1 method also performs well for high and moderate opacities, but not for a small one (green). The LB method, on the other hand, also yields the correct result in this 

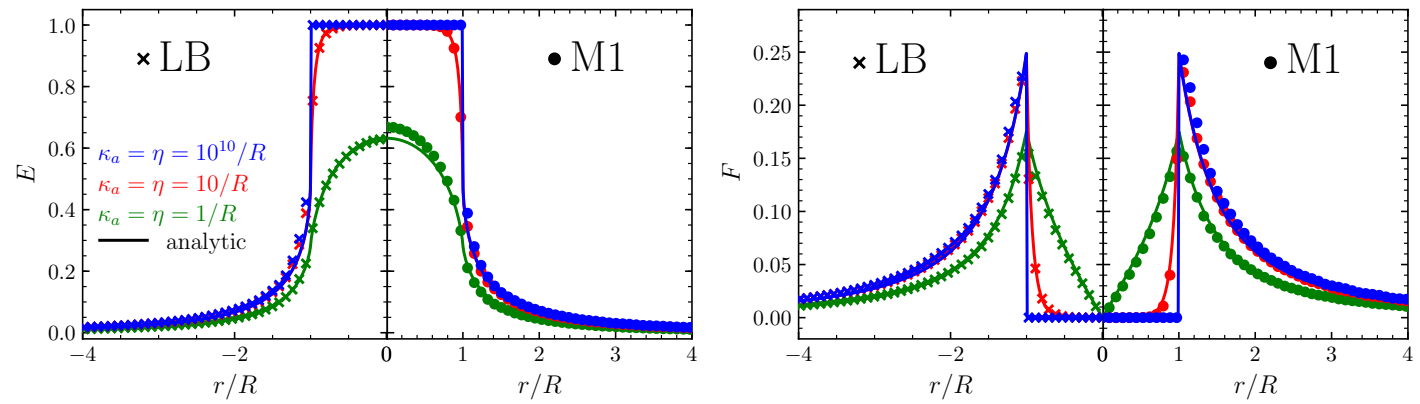

Figure 5.22: Diagonal profiles for the equilibrium of the radiating-sphere test. The left panels show the radiation energy density and the right ones the magnitude of the momentum density. Different colors compare the analytic solutions (solid lines) to the numerical results obtained with LB (crosses) or M1 (dots). Reprint of Fig. 8 in Weih et al. (2020a).

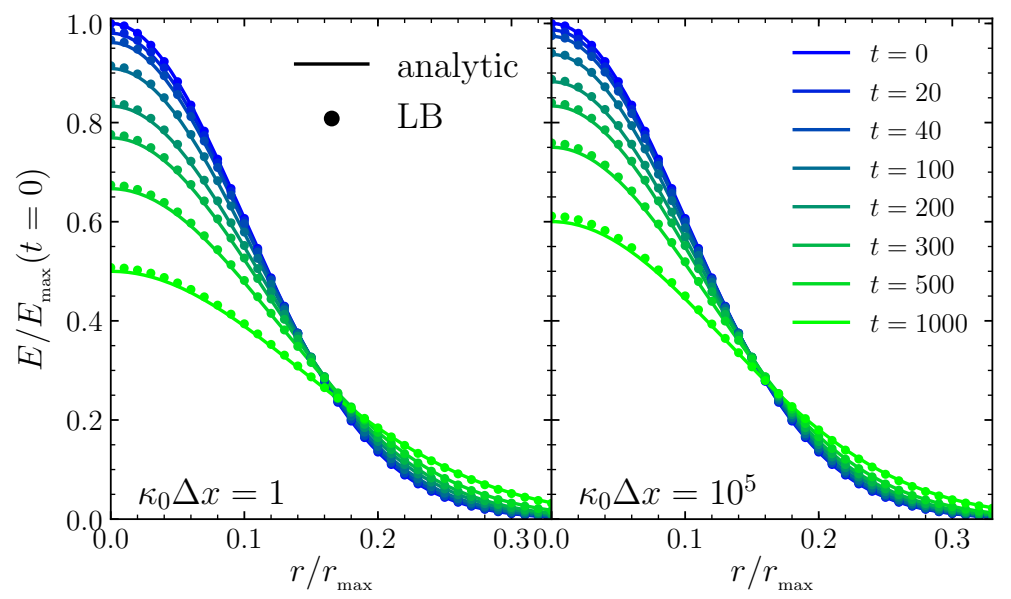

FIGURE 5.23: Diagonal profiles of the radiation energy density for the scattering wave test. The dots compare the numerical solution at different times with the analytic solution (solid lines). The two panels show the case for a low (left) and high (right) Peclet number. Reprint of Fig. 9 in Weih et al. (2020a).

case. This is due to the fact that the LB method does not rely on a closure method and is thus equally accurate for all regimes. Especially in the intermediate regime, where M1 only interpolates the pressure tensor, LB is much more accurate, which is exactly the regime, in which the green case falls.

\section{Diffusion Wave}

Finally, we also test scattering via the same test as for the M1 method, i.e., the diffusion wave test (Pons et al., 2000; O'Connor, 2015). We recall that the initial delta function is avoided by initializing the radiation energy density as

$$
E(r, t=0)=A \exp \left(-\frac{\left(r-r_{0}\right)^{2}}{2 \sigma_{0}^{2}}\right),
$$

where $A, r_{0}$, and $\sigma_{0}$ are the initial amplitude, local center, and width of the Gaussian energy distribution. For the LB method we simply distribute this energy density over all populations weighted by $w_{(i)}$. 


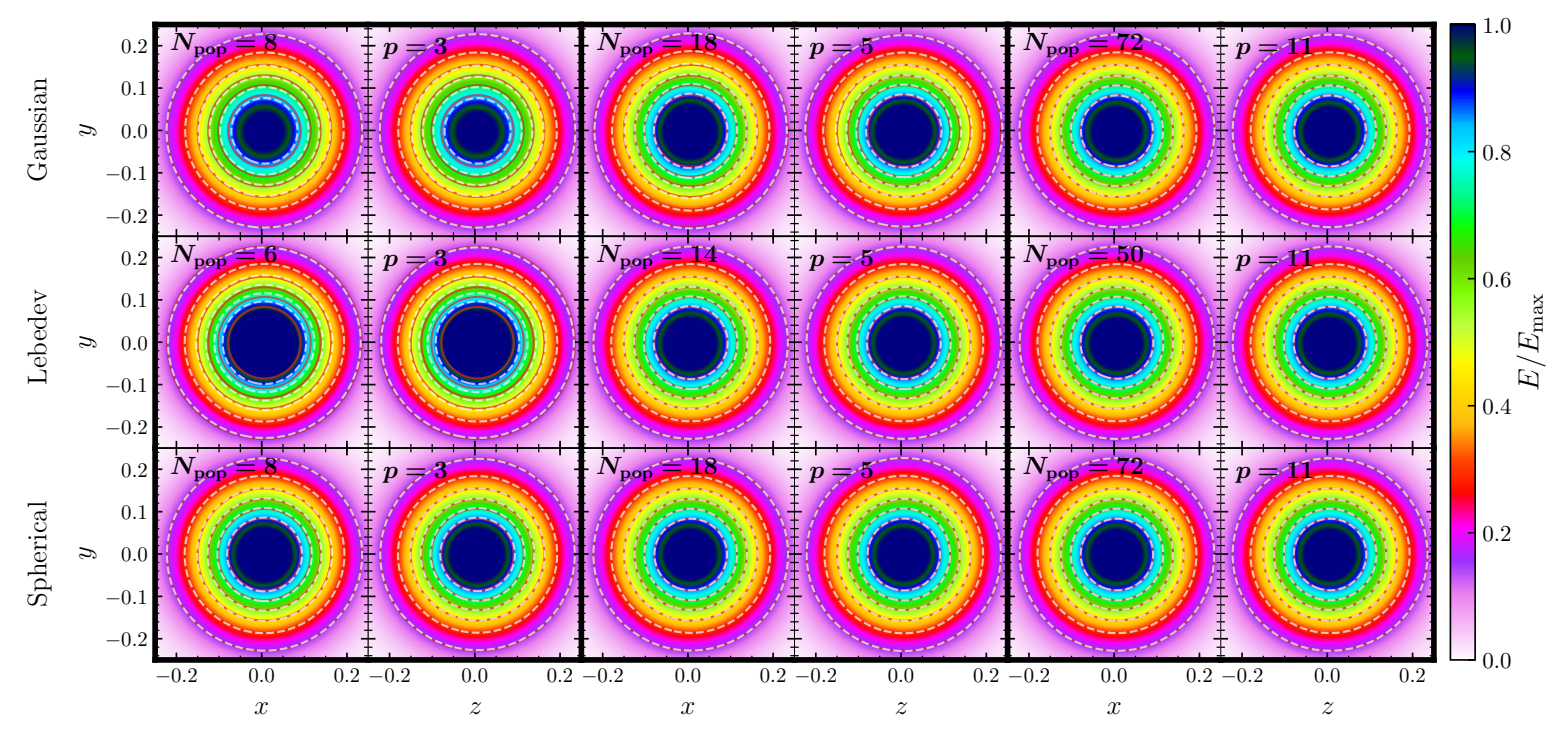

Figure 5.24: Same as Fig. 5.20, but in the diffusion regime. Contour lines compare the analytic solution (solid red) given by Eq. (5.127) with the numerical solution (white dashed). Reprint of Fig. 10 in Weih et al. (2020a).

For a uniform background with no emission or absorption the evolution then follows

$$
E(r, t)=A \frac{\sigma_{0}^{2}}{\sigma_{0}^{2}+\sigma_{D}^{2}} \exp \left(-\frac{\left(r-r_{0}\right)^{2}}{2\left(\sigma_{0}^{2}+\sigma_{D}^{2}\right)}\right)
$$

with $\sigma_{D}=\sqrt{2 D t}$. Within the LB scheme the macroscopic diffusion coefficient $D$ is modeled via the microscopic scattering opacities $\kappa_{0}$ and $\kappa_{1}$. These quantities can be linked via an asymptotic analysis also known as Chapman-Enskog expansion (Chapman \& Cowling, 1970). This link and the consequent choices for $\kappa_{0}$ and $\kappa_{1}$ are derived in Appendix C.

We perform two simulations, one for low Peclet number, i.e., $\kappa_{0} \Delta x=1$ and one with $\kappa_{0} \Delta x=10^{5}$. For both cases we set $\lambda:=3 \kappa_{1} / \kappa_{0}=0.5$. We choose a twodimensional grid with $100 \times 100$ cells. The results are shown in terms of $E(r, t)$ in Fig. 5.23. In both cases the diffusion rate is reproduces correctly.

We repeat the same test also in three dimensions with Peclet number 1. As done for the free-streaming wave test, we again test all three types of quadratures at different orders. The results are compared in Fig. 5.24. In contrast to Fig. 5.20, we can now see perfect symmetry even at small orders. Specifically, we find accurate results with stencils with as few as $N_{\text {pop }} \sim 6-8$ populations. This highlights the fact that LB performs extremely well in the diffusion regime, which is the regime for which it was originally designed. In fact, the performance in the diffusion regime is so good that the type of quadrature has no impact on the accuracy of the results.

\section{Performance}

Having verified that the new LB scheme simulates the correct physics, the question of how efficiently it does so emerges. The benchmark here is the M1 method, which we aim to replace by LB. We have already shown that LB outperforms M1 in various scenarios. Now it is left to show that this does not come at an increased computational cost.

We start by assessing the parallel efficiency of the code. Classical LB is known for 


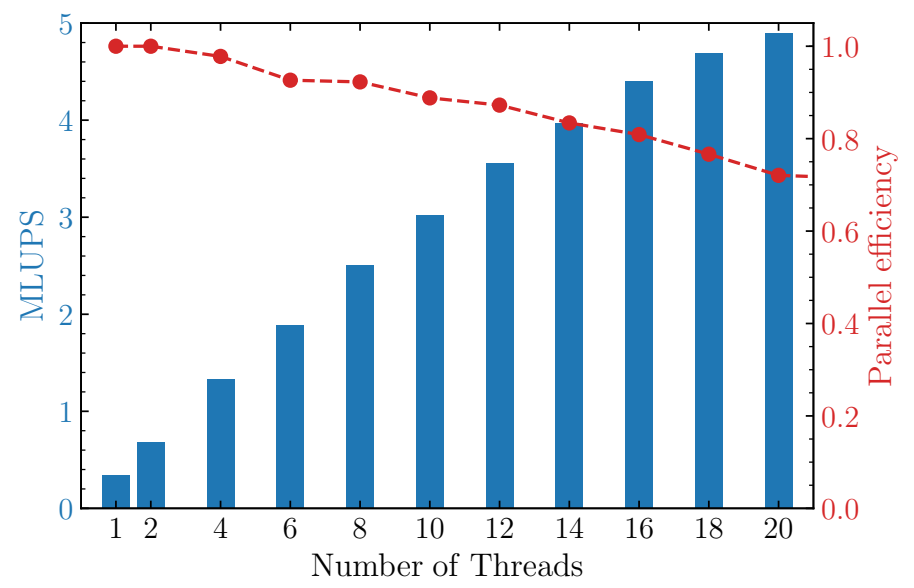

Figure 5.25: Scaling of the LB method on a single 20-core Skylake node. Shown is the performance in terms of MLUPS [see Eq. (5.128)] and the efficiency as function of the number of threads. Reprint of Fig. 13 in Weih et al. (2020a).

its universal applicability on modern computer clusters due to its efficient algorithmic flow stemming from the stream-and-collide paradigm. Details on parallel LB can be found, e.g., in Mazzeo \& Coveney (2008); Bernaschi et al. (2009, 2010); Godenschwager et al. (2013); Succi et al. (2019). W.r.t. classical LB our new method has two major changes that might impact the performance. First, while still adopting the stream-and-collide approach, we use off-lattice stencils requiring interpolation. Second, the number of populations in our new scheme is significantly higher than for classical LB.

We assess the performance in terms of Million Lattice Updates per Second (MLUPS) defined as

$$
\text { MLUPS }=10^{6} \frac{L^{3} N_{\text {iter }}}{t_{\text {exec }}} .
$$

According to this definition, MLUPS means that on a cubic grid of size $L^{3}$ simulating $N_{\text {iter }}$ many iterations requires a time $t_{\text {exec }}$. We fix the problem size, $L=128$, and the number of iterations, $N_{\text {iter }}=200$, for the emitting-sphere test with a $p=20$ sphericaldesign stencil. Fixing these parameters, we expect that increasing the number of computation cores ideally reduces $t_{\text {exec }}$ linearly and thus increases MLUPS.

We test the single-node performance on a single Intel Skylake 20-core processor. For the parallelisation we use OpenMP directives, which allow for easy portability. The results are shown in Fig. 5.25. As expected the measure of MLUPS increases with an increasing number of threads. We find a single-node efficiency of $\gtrsim 70 \%$ up to 20 cores. While these numbers are already good, there is still space for improvements. The limiting factor is the memory access, which ideally should always fully exploit the available cache and vector units of the processor. Typically, LB employs two different strategies for achieving this. The array of structures (AoS) memory layout stores all $N_{\text {pop }}$ populations contiguously at every grid point. The structure of arrays (SoA) layout, on the other hand, stores all grid points for one population $(i)$ contiguously, while different populations at the same grid point are stored far away from each other. There are also more modern and sophisticated approaches (Shet et al., 2013; Calore et al., 2019) that should be explored for our new LB scheme in the future.

Another limiting factor for the LB method is the number of populations. The advantage of classical LB is that it achieves a high accuracy already with a small number of discrete velocities. As shown previously the free-streaming regime requires a rather large number of velocities. We show in Fig. 5.26 how the performance 


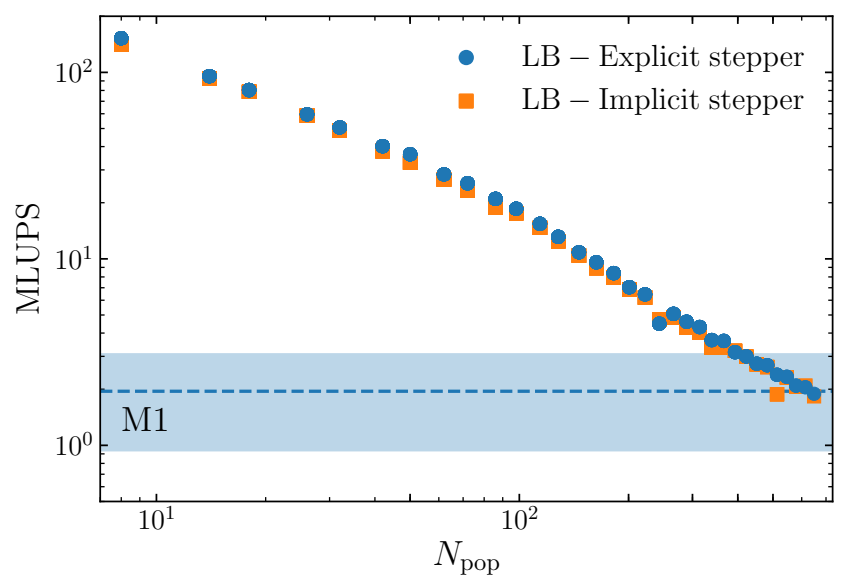

Figure 5.26: Performance of the LB method as function of $N_{\text {pop }}$. We compare the runtime when using an implicit Lambda-iteration method (orange squares) with that of an explicit Euler time stepper (blue dots). Also shown as comparison is the performance of the M1 method for the same standard test. The blue shaded region corresponds to a range that is set by the accuracy of the closure. The dashed line corresponds to a reasonable standard choice for this accuracy. Reprint of Fig. 14 in Weih et al. (2020a).

decreases as a function of $N_{\text {pop }}$. Shown are the results for the implicit solver (orange squares) and a simple explicit Euler solver (blue dots). For having consistent results we fix the number of cycles within the Lambda iteration to five. Since the code is completely memory bound, the difference in runtime between implicit and explicit method are negligible.

The same figure also shows the performance of M1 for the same test as comparison. Note that the LB code and the special-relativistic version of FRAC feature a similar level of optimizations. For the M1 method the runtime is mostly bound by the closure, which requires a root-finding (see Sec. 5.1.1). The accuracy of this closure then determines the runtime. We show results for a standard setup, i.e., with a relative accuracy of $10^{-9}$ for the closure, as dashed line; the blue shaded region corresponds to the performance when considering higher $\left(10^{-14}\right)$ or lower $\left(10^{-4}\right)$ accuracy. In any case, the comparison with LB shows that the LB method outperforms M1 for $N_{\text {pop }} \lesssim 400$. Only above this value does LB become computationally more expensive. Considering that $N_{\text {pop }} \approx 400$ has been shown to be enough populations for achieving high accuracy even in the free-streaming regime, indicates that the new LB method is indeed feasible.

This is especially true when considering that LB is particularly well suited for a GPU implementation (Bernaschi et al., 2010; Calore et al., 2016), which might yield an order of magnitude improvement in terms of performance. We have tested a GPU-optimized version of our code on an NVIDIA V100 GPU by implementing simple OpenACC statements. Indeed, we found a consistent speed-up of roughly one order of magnitude for $N_{\text {pop }} \in[0 ; 200]$. Beyond that value no more tests could be performed due to the limited memory provided by the GPU, which is a general problem of such a GPU implementation.

\subsubsection{Relativistic Jets}

We have shown that the LB method is accurate in all physical regimes relevant for numerical astrophysics simulations. Furthermore, we have shown that this comes at 
a reasonable computational cost. The final step for establishing this new method is a realistic simulation, where the radiation is coupled to a dynamically evolving fluid.

We do so by simulating relativistic jets, which are important in the study of active galactic nuclei (Perucho, 2019), where supermassive black holes produce jets through accretion. This is a commonly modeled problem in relativistic astrophysics (see Porth et al., 2019, for an overview). Usually radiation is only introduced during postprocessing and only Rivera-Paleo \& Guzmán (2019) simulated such jets with radiation dynamically reacting with the fluid via an M1 scheme. SGRBs are also associated to the merger of two neutron stars, where the strong magnetic field together with radiative interactions might cause such highly energetic jets (Rezzolla et al., 2011).

We simulate this problem by coupling our new LB code to BHAC (Porth et al., 2017). Technically, the coupling is almost the same as for the M1 method with the difference that instead of the evolved moments we now introduce $N_{\text {pop }}$ new evolved variables to BHAC, one for each intensity population. In summary the total procedure is as follows:

1.) At every iteration we pass the fluid rest-mass density, $\rho$, and the temperature, $T$, together with the fluid three-velocity, $v^{i}$, to the LB code. From these variables we compute the opacities and emissivity as will be detailed below.

2.) While BHAC advances the conservative fluid variables, the LB code solves Eq. (5.109) as detailed in Sec. 5.2.2.

3.) From the updated populations the LB code computes the zeroth, first and second moment.

4.) From these moments the collisional sources $S_{0}=W\left(\tilde{\kappa}_{a} J-\tilde{\eta}\right)+\tilde{\kappa} H_{0}$ and $S_{j}=$ $W\left(\tilde{\kappa}_{a} J-\tilde{\eta}\right) v_{j}+\tilde{\kappa} H_{j}$ can be computed, which are returned to BHAC. We recall that $W$ is the Lorentz factor and $J$ and $H_{\mu}$ are the first two moments in the comoving fluid frame. In special relativity they can be obtained from the moments in the Eulerian frame via

$$
\begin{aligned}
J & =W^{2}\left(E-2 F^{i} v_{i}+P^{i j} v_{i} v_{j}\right), \\
H_{j} & =W^{3}\left(F^{i} v_{i}-E\right) v_{j}+W h_{i j} F^{i}-W h_{i j} v_{k} P^{i k},
\end{aligned}
$$

where $h_{i j}=W^{2} v_{i} v_{j}+\delta_{i j}$ is the projection operator orthogonal to the fluid velocity. The component $H_{0}$ can be obtained from the condition $H_{\mu} u^{\mu}=0$.

5.) Within BHAC these sources are then simply subtracted from the energy and momentum equations, respectively, which completes the time step.

The missing ingredient is the computation of the opacities and emissivity. Since the LB equation (5.109) is written in the Eulerian (lab) frame, we also need to compute the microphysical parameters $\kappa_{a}, \kappa_{0}, \kappa_{1}$, and $\eta$ in this frame. However, the microphysical derivation of these quantities is only well defined in a frame, where the fluid is at rest, i.e., in the fluid frame. We thus compute the corresponding quantities in the fluid frame, $\tilde{\kappa}_{a}, \tilde{\kappa}_{0}, \tilde{\kappa}_{1}, \tilde{\eta}$ and then transform them to the corresponding quantities in the lab frame. This is known as mixed-frame scheme (see for example Mihalas \& Auer, 2001) and how this transformation works is outlined in Appendix B.

For the general simulation setup we follow Martí et al. (1997), who have extensively analyzed relativistic jets in the purely hydrodynamical case. Within this setup the jet is injected through a circular nozzle of radius $r_{\text {jet }}=1$ at the bottom of the domain of a Cartesian grid. The fluid velocity is chosen to point in z-direction, so 


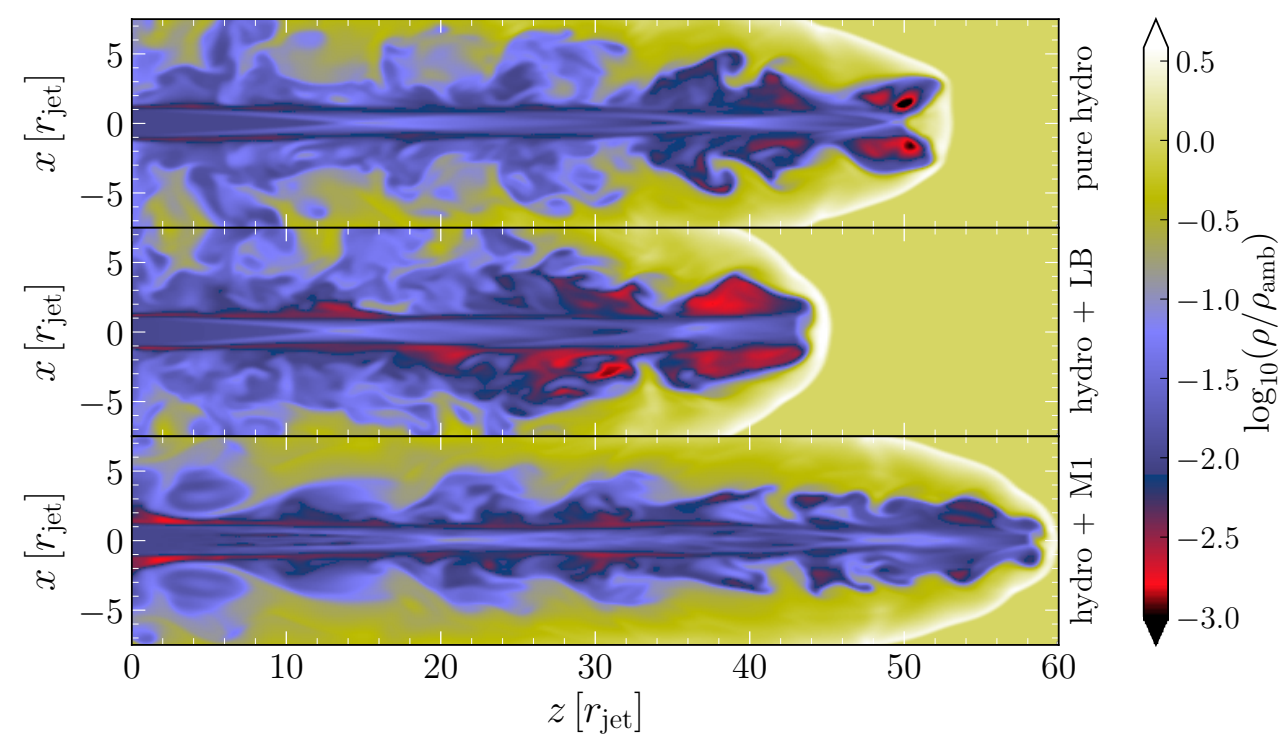

Figure 5.27: Normalized rest-mass density of a relativistic jet after $t=125 r_{\text {jet }}$. The panels show the results for a purely hydrodynamical simulation (top), and a coupled hydro-radiation simulation where the radiation is treated with either LB (middle) or M1 (bottom). Reprint of Fig. 11 in Weih et al. (2020a).

that the jet will travel from bottom to top parallel to the z-axis. For this setup there are four free parameters:

1.) The Newtonian Mach number, $\mathcal{M}:=v_{\text {jet }} / c_{s}$, where $c_{s}$ is the local speed of sound and $v_{\text {jet }}$ the velocity of the jet.

2.) The Lorentz factor of the jet, $W_{\text {jet }}=\sqrt{1-v_{\text {jet }}^{2}}$.

3.) The ratio of the rest-mass density inside the jet to that of the ambient medium, $\mathcal{R}:=\rho_{\text {jet }} / \rho_{\text {amb }}$.

4.) The ratio of the pressure inside the jet to that of the ambient medium, $\mathcal{K}:=$ $p_{\text {jet }} / p_{\text {amb }}$.

Here, we simulate a simple pressure-matched jet, for which $\mathcal{K}=1$. For the other parameters we choose $\mathcal{M}=42, W_{\text {jet }}=7$, and $\mathcal{R}=0.01$. All evolved variables can be initialized from these parameters together with an ideal-gas EOS.

Note that this setup is not the most sophisticated one. A more realistic scenario with a non-homogeneous ambient medium is simulated in Fromm et al. (2018). In order to simulate a truly three-dimensional problem (which is an important test for our new LB method) we include the helical perturbation introduced in Aloy et al. (1999). This leads to a jet that is no longer axisymmetric.

The radiation - in this case photons produced by the hot fluid - computed within our LB scheme lives on a Lebedev stencil with $N_{\text {pop }}=154$. We initialize all intensities, $I_{(i)}$, as zero and let the photons be produced self-consistently by the fluid. For this we impose a black-body emissivity, i.e.,

$$
\tilde{\eta}=\frac{\sigma_{\mathrm{SB}}}{\pi} \tilde{\kappa}_{a} T^{4},
$$

with an absorption opacity $\tilde{\kappa}_{a}=\rho^{2} T^{-3.5}$. The functional form of this opacity is inspired by thermal bremsstrahlung (Rybicki \& Lightman, 1986, see also Eq. (5.75)), 

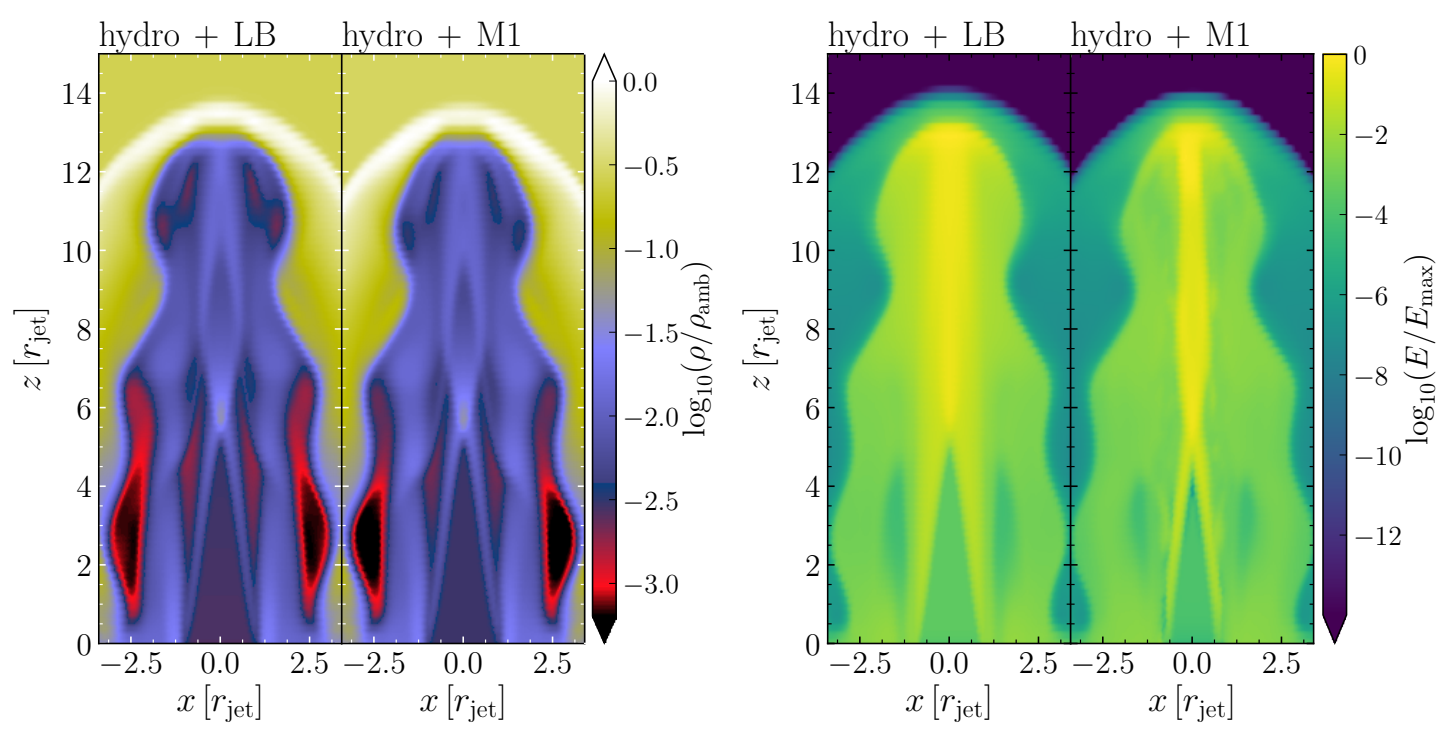

Figure 5.28: Close-up of the jet after $t=30 r_{\text {jet }}$. The left panels show the fluid rest-mass density for LB (left) and M1 (right). The right panels show the radiation energy density of the same snapshot. Reprint of Fig. 12 in Weih et al. (2020a).

but in arbitrary units. These arbitrary units are set by the choice of the numerical value of the Stefan-Boltzmann constant, $\sigma_{\mathrm{SB}}$, and the density of the ambient medium, $\rho_{\mathrm{amb}}$. Instead of physically realistic values, we choose $\rho_{\mathrm{amb}}=1$ and $\sigma_{\mathrm{SB}}=0.1$, which allows us to control the amount of radiation. Specifically, the chosen values lead to a jet with a moderate amount of radiation that is neither fluid nor radiation dominated. Scattering is added by $\tilde{\kappa}_{0}=10^{-3} \rho$ and $\tilde{\kappa}_{1}=0$. This choice is motivated by Thomson scattering, for which the opacity is proportional to the number of scatters in the medium (hence, the choice for $\tilde{\kappa}_{0}$ ) and which is isotropic (hence, the choice for $\tilde{\kappa}_{1}$ ).

Let us first consider the purely hydrodynamical case (without any radiation coupling), for which the result after a evolution time $t=125 r_{\text {jet }}$ is shown in the top panel of Fig. 5.27. The morphology of this jet with its different kind of shocks is well understood and is the same for our jet as was found in Martí et al. (1997); Aloy et al. (1999). The same jet, when coupled to the LB code, is shown in the middle panel of the same figure. Clearly, this jet propagates slower than the purely hydrodynamic one. Specifically, we find a Lorentz factor that is $\sim 15 \%$ smaller. This makes sense when considering that the fluid produces radiation and thus loses some of its energy to the radiation field.

For verifying the correct behavior of the radiation-coupled jet, we also simulate the same setup with the M1 method (using FRAC) instead of LB coupled to BHAC. We use the same opacities and emissivity and this time initialize the radiation energy and momentum density to zero. Surprisingly, the results shown in the bottom panel of Fig. 5.27 significantly differ from the LB results.

The cause of this difference can be seen when looking at the radiation energy density. We show this in the right panels of Fig. 5.28 at an earlier time, i.e., at $t=$ $30 r_{\text {jet }}$. At this time one can see particularly well what is the reason for the different propagation speeds between M1 and LB. While the fluid (left panels) looks almost identical at this point, the radiation energy density starts to differ. In both cases there is a triangular region of low energy. This region comes from the recollimation shock produced by the injection of the jet (Aloy \& Rezzolla, 2006; Mizuno et al., 2015). Around this region, one can see on either side beams of increased radiation energy. In the case of M1, these beams merge at the peak of the recollimation shock 
and form a single beam of increased energy density along the z-axis. This is the same unphysical phenomenon as analyzed for the crossing-beam problem in Sec. 5.2.3. This artificially enhanced radiation then exerts an additional pressure on the fluid causing the jet to propagate faster. In the case of LB, on the other hand, this merging is not present and instead the energy density above of this triangular region is distributed much more uniformly. This wide distribution is also what causes the LB jet to be less pointed. While this difference in the radiation does not yet have a visible effect at $t=30 r_{\text {jet }}$, it accumulates during the simulation, eventually leading to drastically different propagations.

\subsection{Summary and Outlook}

In this chapter two methods have been presented for radiative transport in numerical high-energy astrophysics. The first method is the commonly used M1 method and the second one the radiative Lattice Boltzmann method.

First, an implementation of the M1 method was presented in Sec. 5.1. The implementation was verified via a number of standard tests and applied to the radiative Michel problem. A comparison to the existing literature yielded good agreement (Fragile et al., 2012; Roedig et al., 2012; Sądowski et al., 2013; McKinney et al., 2014). A large parameter space was explored, from which a simple relation could be derived that allows to compute the accretion efficiency from observables like the black hole mass or its luminosity [see Eq. (5.85)]. Furthermore, in Sec. 5.1.3 was shown for the first time that the radiative Michel solution is non-linearly stable under perturbations of the radiation field.

FRAC implements the M1 method in full general-relativity, handling stationary as well as dynamic spacetimes. Furthermore, it takes into account an accurate closure for the optically thick and thin limit with a reasonable interpolation in the intermediate regime. Together with the IMEX for handling the stiff source terms all ingredients are present that are required for simulations of binary neutron star mergers. Having this type of simulations in mind for future applications, FRAC has also been coupled to the Einstein Toolkit (ET), which provides a computational infrastructure for all kinds of high-energy astrophysical simulations (Loeffler et al., 2012). It offers a modular framework via a code basis called flesh, to which physics modules, so-called thorns, can be coupled. For coupling FRAC the same SS2 $(2,2,2)$ IMEX method (Pareschi \& Russo, 2005) as presented in Sec. 5.1.1 has been implemented in the thorn MoL, which is responsible for the time update within the ET. Furthermore, FRAC has been extended to evolve the radiation variable for not only one, but three species corresponding to electron, anti-electron and heavy neutrinos, where the latter includes a combination of myon, anti-myon, tau, and anti-tau neutrinos. The coupling between FRAC and the thorn responsible for the evolution of the hydro-variables is the same as the coupling with BHAC outlined in Sec. 5.1.1 with the difference that the collisional source terms of the three neutrino species are added to a single collisional source term for the corresponding conserved fluid variables. Furthermore, the collisional source term for the electron fraction, i.e., Eq. (5.61), is also provided. The reactions are implemented as in Foucart et al. (2015), which in turn is based on Ruffert et al. (1996); Burrows et al. (2006); Rosswog \& Liebendörfer (2003). For this we use the thorn Margherita, which already implements these reactions for a Leakage scheme (Most et al., 2019b). This coupling of FRAC and the ET will allow state-of-the-art binary neutron star merger simulations in the future. 
It was also shown that M1 suffers from inaccuracies that are intrinsic to the method. One major problem is the unknown pressure tensor, which is only provided via an ad-hoc closure relation. It was shown that this leads to an incorrect radiation field in the regime between the optically thick and thin limit. Furthermore, the merging of two radiation beams has been demonstrated as unphysical behavior. This can potentially have drastic effects in simulations of binary neutron stars. Indeed, in such a system radiation will be emitted from the edges of the torus towards the poles, where they meet and incorrectly merge when using the M1 method. Unfortunately, a correct treatment of the radiation at the merger remnant's poles is particularly important. It is connected to the production of SGRBs from such a merger, which is believed to be only possible with the correct treatment of magnetic fields and neutrino radiation (Fujibayashi et al., 2017).

Since more accurate methods for radiative transport are computationally unfeasible (at least in three dimensions), a new accurate and feasible method has been presented in Sec. 5.2. This radiative LB method is a variation of the classical LB method and has been adapted for numerical astrophysics by taking into account stiff source terms, coupling to a moving fluid, and a physical propagation of radiation. It has been shown in Sec. 5.2.3 that the new LB method does correctly pass all standard tests and in some cases even outperforms the M1 method at a comparable computational cost. Furthermore, we performed the first astrophysically realistic simulation that makes use of the LB method. These simulations of relativistic jets provide not only a proof of concept for the applicability of the LB method in numerical astrophysics, but also further revealed the systematic inaccuracies of the M1 method. While this is only a first step, more work has to be put into the development of this new method. Specifically, an extension to general relativity is indispensable for an application to black holes and neutron stars. For doing so, the streaming step has to be generalized to a curved spacetime. Since an interpolation is already required for the streaming, this should not introduce any further complications. Also a gravitational source term has to be added on the RHS of the LB equation. The starting point for deriving these gravitational source terms should be the general-relativistic Boltzmann equation in $3+1$ form (Cardall et al., 2013) making a general-relativistic LB scheme compatible with Einstein solvers written in $3+1$ form (see Sec. 2.1.2).

Further improvements concern adaptive mesh refinement, for which streaming from coarse to fine (and vice versa) has to be accounted for. For an extension to a frequency-dependent scheme one would have to tackle the same obstacles and accept the same increase in computational cost as for the M1 method. The same can be said about the inclusion of more complex (beyond iso-energetic) scattering processes (see also the appendix of Bruenn, 1985, for a summary of these scattering processes). 


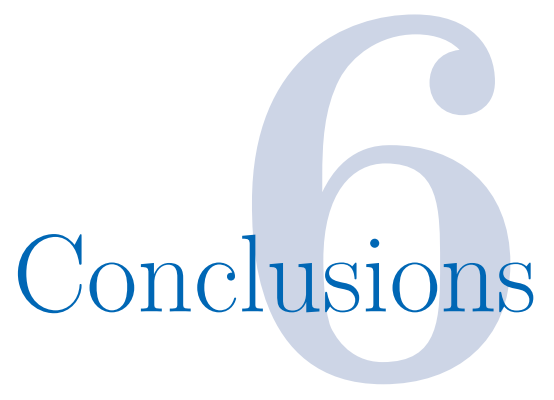

In this Thesis a number of results have been presented in three chapters that will be summarized here. Chronologically the work on this Thesis started with GW170817. This is also the starting point in Chap. 3. Based on Rezzolla et al. (2018a) it was shown how the multimessenger signal of GW170817 could be used to set an upper bound on the maximum mass of neutron stars. This upper limit was confirmed by several groups (Margalit \& Metzger, 2017; Ruiz et al., 2018a; Shibata et al., 2017) and has since then been revisited with a robust value of $\sim 2.3 M_{\odot}$ (Shibata et al., 2019). Furthermore, the neutron star radius could be constraint following the measurement of the inspiraling neutron stars' tidal deformability. These constraints have been presented in a flood of publications and are summarized in Tab. 3.1. All this information could be translated on constraints on the EOS of nuclear matter as shown in Fig. 3.11. In general, conclusions from GW170817 indicate that softer EOSs are favored.

On the other hand, the direct observations of the NICER mission favor rather stiff EOSs, but still in agreement with GW170817 within a $2 \sigma$ error range. Given that both type of observations will become more common and more accurate in the future, we also present a combination of the corresponding constraints in Sec. 3.3 based on Weih et al. (2019). Simulating all possible radius measurements coming from future X-ray observations, it was shown that in combination with the currently known constraints, the biggest improvement could be achieved when measuring the most massive stars. For the lower density regime of the EOS also neutron stars with $\sim 1.8 M_{\odot}$ were found to be promising candidates for future observations. The dataset used for this analysis is the largest of its kind to date and is publicly available (Weih et al., 2019b). Once future detections are available (from gravitational or electromagnetic waves) this dataset can be used to directly translate these observations into constraints on the EOS. In the most optimistic scenario, astrophysical observations together with theoretical modeling will be able to constrain the EOS to a line in the $p-\rho$ plane given enough future observation. This would provide the exact EOS for densities up to $\sim 6-7$ times nuclear saturation density. However, the fundamental physics that leads to this exact EOS will then still be unknown, which is the point where nuclear physics needs to take over.

The next chapter, i.e., Chap. 4 focused on the postmerger signal of binary neutron star mergers. While this signal is already well understood for the classical case of purely hadronic mergers (Baiotti \& Rezzolla, 2017; Dietrich et al., 2020b), this is not the case for the scenario where a quark-hadron PT occurs during the merger. Only recent simulations (Most et al., 2019a; Bauswein et al., 2019) with new realistic EOSs that allowed for such a PT made the modeling of this scenario possible. The presentation of the DPT scenario (Weih et al., 2020b) completed the picture of possible merger outcomes in the presence of a PT. This scenario was analyzed in detail and the corresponding gravitational-wave signal was presented. 
This signal shows a clear signature for the occurrence of the PT (see Fig. 4.9) in form of a double-peak structure in the total power spectrum. Future detectors with increased sensitivity at high frequencies might be able to pick up this signal (Barsotti et al., 2020; Punturo, 2020; Punturo et al., 2010; Abbott \& et al., 2017). If the DPT scenario is indeed the one realized in nature, then the detection with a signature as the one presented in this Thesis will be the evidence for the creation of quark matter in our universe.

A systematic study via numerical simulations will reveal more details and potentially even universal relations in these signatures (see Blacker et al., 2020; Bauswein \& Blacker, 2020, for first studies in this direction). Currently, these studies are limited by the availability of suitable EOSs. In fact the EOSs used in Most et al. (2019a); Bauswein et al. (2019) are the only temperature-dependent EOSs that include a PT and are suitable for binary neutron star simulations. While waiting for more such EOSs to become available, the hybrid approach used in Chap. 4, where the cold part of the EOS can be suitably adjusted for the desired outcome of the merger, presents a viable approach. In fact, Figura et al. (2020) has recently shown that hybrid EOSs yield results comparable to these of tabulated temperature-dependent EOSs. This hybrid approach then allows for a systematic study of a large parameter space for the PT. Optimistically, gravitational-wave detections well then not only reveal whether there is a PT or not, but also shed light on the PT's properties like the onset density or the jump in energy.

In the final chapter of this Thesis, i.e., Chap. 5, two methods for radiative transport suitable for numerical astrophysics have been presented. The M1 method and its implementation in FRAC have been detailed based on Weih et al. (2020c). This method is already used in astrophysical simulations. Here it was applied to spherically symmetric accretion onto black holes revealing a relation [Eq. (5.85)] that relates the black hole accretion efficiency to astrophysical observables. FRAC provides a new infrastructure that allows for future simulations of different astrophysical systems (black hole-torus or binary neutron star systems) with the inclusion of a sophisticated treatment for radiation (in form of photons or neutrinos). These simulations will be of great importance for the understanding of the long-term evolution of the merger remnant of two neutron stars. Together with an accurate treatment of magnetic fields (see Most, 2020) these simulations might reveal the mechanism responsible for the production of SGRBs. Fortunately, since GW170817 we have definite proof that neutron star binary mergers are connected to these jets (LIGO Scientific Collaboration et al., 2017). However, such a jet has never been produced in a self-consistent way in a simulation ${ }^{1}$. Due to numerical relativity codes becoming more and more sophisticated, to which FRAC is a small contribution, it is merely a question of time until the first simulations will show the production of an SGRB.

The second method presented in Chap. 5 is the radiative LB method based on Weih et al. (2020a). The development of this method was motivated by the fact that currently used radiative transport methods are either too inaccurate or computationally unfeasible. This new method is based on the classical LB method, which is known for its efficiency (Calore et al., 2016) and at the same time provides a numerical solution to the Boltzmann equation, the governing equation also for radiative transport. While the exact recovery of moments from a low-order quadrature had to be abandoned due to the lack of suitable conservation laws, a number of quadratures

\footnotetext{
${ }^{1}$ Mildly relativistic jet-like structures have been observed when seeding a strong magnetic field by hand right before merger (Paschalidis et al., 2015; Ruiz et al., 2018b, 2020a), while in other cases even this artificial magnetic field is not sufficient to drive a relativistic outflow (Ruiz et al., 2020b).
} 
based on isotropy conditions was analyzed for the new radiative LB scheme. Furthermore, the efficient stream-and-collide approach could also be used for this new method. As a first step, the derivation presented in this Thesis is gray and restricted to special relativity. Nonetheless, it could be shown that LB outperforms the M1 method. Two major improvements are the correct crossing of radiation beams and the correct treatment of the intermediate regime between the optically thin and thick limits. A coupling to the GRMHD code BHAC allowed for the first LB simulations with a dynamic fluid background. On one hand, these simulations proof the applicability of LB in high-energy astrophysics. On the other hand, they further revealed the deficiencies intrinsic to the M1 method. These results together with the runtime comparison at the end of Sec. 5.2.3 raise hope that this new method might become widely used in future simulations of not only binary neutron star mergers, but in general for all kinds of astrophysical scenarios. For this to happen, however, an extension to general relativity is strictly necessary.

As mentioned numerous time, this Thesis greatly benefited from the gravitationalwave event GW170817 marking the beginning of the modern multimessenger era. Since GW170817 at least one more neutron star binary merger, i.e., GW190425 (Abbott et al., 2020a), has been detected by LVC. Unfortunately and in contrast to GW170817, this event could only be detected in gravitational waves and without an electromagnetic counterpart. Consequently, much less insights could be gained from this event and a lot is left to speculation (Most et al., 2020c; Han et al., 2020; Kyutoku et al., 2020; Romero-Shaw et al., 2020; Safarzadeh et al., 2020). In fact, LVC just ended their third observation run and published the results of the run's first six months (Abbott et al., 2020b) shortly before the conclusion of this Thesis. Improving their detectors in preparation of the next run, they project a bright future of gravitational-wave astronomy (The LIGO Scientific Collaboration \& the Virgo Collaboration, 2020). The results obtained within this Thesis will hopefully contribute to this future. 


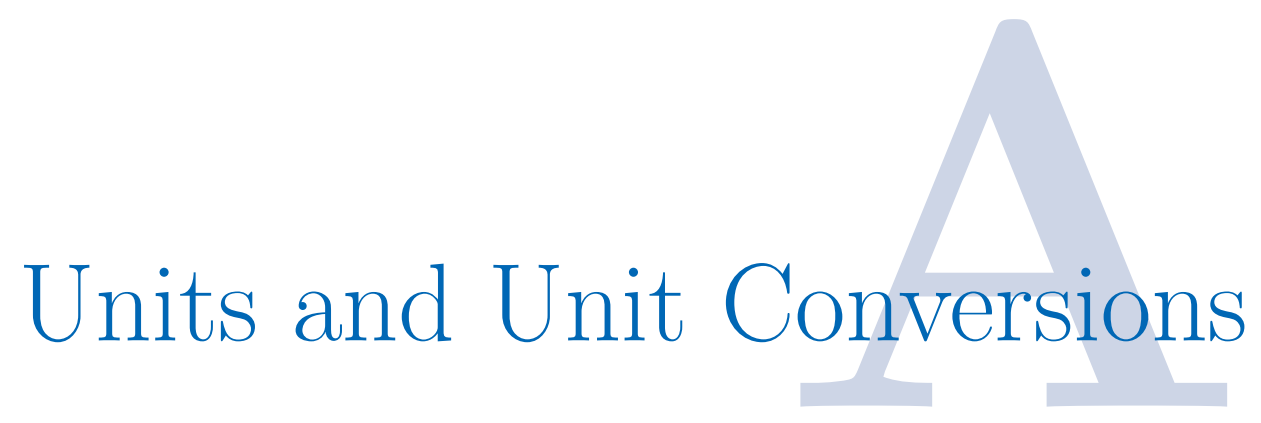

In Sec. 5.1 the radiative transfer code FRAC is introduces and coupled to the GRMHD code BHAC in order to perform GRRTMHD simulations in Sec. 5.1.3. One technical problem that arises from this coupling concerns the system of units. When coupling radiation with matter, at some point we have to introduce information about the scale between the two. In other words, we have to prescribe the ratio between the mass of, e.g., a proton, and the mass of the plasma. In a GRMHD simulation without radiation this is not necessary, which makes these simulations scale-invariant. This invariance is broken by the introduction of radiation.

For describing the unit system we start by recalling that within this entire thesis and also within BHAC units with $c=G=1$ are used. Within BHAC these geometrized units together with the black hole mass determining the stationary metric result in a conversion factor for the length, i.e.,

$$
[L]=\frac{G M}{c^{2}},
$$

where we use the convention of writing units in square brackets. Together with the trivial unit conversion for velocity, $[V]=c$, one can derive the conversion for time as $[T]=[L] / c$. Evidently, the length and time units scale with the black hole mass and one typically simply uses $M=1$.

Strictly speaking, $M$ would set the mass scale. This, however, would lead to numerical values of $\mathcal{O}\left(10^{-22}\right)$ for the fluid, which is below floating-point precision. For avoiding such small values for the evolved variables, we make use of the fact that the torus around the black hole is much lighter than the black hole itself and does not contribute to the spacetime curvature. This allows the definition of an independent mass scale. For the problem simulated in Sec. 5.1.3 it is convenient to derive this mass scale from the Eddington accretion rate, $\dot{M}_{\text {Edd }}$, which in code units becomes one. The mass scale then follows as

$$
[M]=\dot{M}_{\mathrm{Edd}} \times[T] .
$$

All other units can be derived from $[M],[L]$, and $[T]$ except for the temperature, for which we simply use Kelvin, K. The rest-mass density, for example, follows as $[\rho]=[M] /[L]^{3}$.

For easy reference, we here summarize some useful conversions for the type of GRRTMHD simulation performed in Sec. 5.1.3. The frequently used Boltzmann constant and proton mass are converted from CGS to code units as

$$
k_{\mathrm{B}}^{\text {code }}=\frac{k_{\mathrm{B}}^{\mathrm{CGS}}}{[M][V]^{2} \mathrm{~K}}=2.22992 \times 10^{-49}\left(\frac{M}{M_{\odot}}\right)^{-2} \mathrm{~K}^{-1},
$$




$$
m_{\mathrm{p}}^{\text {code }}=\frac{m_{\mathrm{p}}^{\mathrm{CGS}}}{[M]}=2.42798 \times 10^{-36}\left(\frac{M}{M_{\odot}}\right)^{-2} .
$$

Another important quantity is the radiation constant, $a_{\mathrm{R}}:=4 \sigma_{\mathrm{SB}} / c=7.5657 \times$ $10^{-15} \mathrm{erg} \mathrm{cm}^{-2} \mathrm{~K}^{-4}$. This constant enters in the computation of the emissivity. The expressions for the emissivity as well as for the opacities is usually given in CGS units. The conversion to code units follows as

$$
\begin{aligned}
& \eta^{\text {code }}=\eta^{\mathrm{CGS}}\left(\frac{[L]^{2}[T]^{2}}{[M]}\right)=7.67822 \times 10^{-13}\left(\frac{M}{M_{\odot}}\right)^{2} \eta^{\mathrm{CGS}}, \\
& \kappa^{\text {code }}=\kappa^{\mathrm{CGS}} \times[L]=1.47760 \times 10^{5}\left(\frac{M}{M_{\odot}}\right) \kappa^{\mathrm{CGS}} .
\end{aligned}
$$




\section{Emissivity and Opacities in the Laboratory Frame}

The opacities and emissivity required for radiative transfer simulations are usually derived and expressed in the comoving fluid frame, so that the fluid is at rest. The radiative LB equation (5.109), on the other hand, is written in the lab frame, thus requiring the lab frame quantities. These lab frame quantities can be obtained from the fluid ones by a transformation as derived in Mihalas \& Auer (2001). We here summarize this transformation for the gray approximation and specifically for the microphysical quantities used in Sec. 5.2.4. We use a tilde to denote the quantities in the fluid frame and write quantities in the lab frame without tilde, which is the same convention as used in Sec. 5.2.4.

We start by analyzing how the frequency of a radiation particle changes for a moving fluid background. For a fluid velocity $v_{i}$ and Lorentz factor $W$, the lab-frame frequency $\nu$ of a particle propagating in direction $\hat{\boldsymbol{n}}$ is transformed to the fluid frame via

$$
\tilde{\nu}=W \nu\left(1-v_{i} \hat{n}^{i}\right) .
$$

Mihalas \& Auer (2001) then show that the frequency-dependent quantities, which are isotropic in the fluid frame, transform like

$$
\begin{aligned}
\eta_{(\nu)} & =\frac{\nu^{2}}{\tilde{\nu}^{2}} \tilde{\eta}_{(\tilde{\nu})}=\frac{\tilde{\eta}_{(\tilde{\nu})}}{W^{2}\left(1-v_{i} \hat{n}^{i}\right)^{2}}, \\
\kappa_{a,(\nu)} & =\frac{\nu}{\tilde{\nu}} \tilde{\kappa}_{a,(\tilde{\nu})}=W\left(1-v_{i} \hat{n}^{i}\right) \tilde{\kappa}_{a,(\tilde{\nu})} .
\end{aligned}
$$

For the frequency-averaged absorption opacity we then find [see also Eq. (2.93)]

$$
\kappa_{a}=\frac{\int_{0}^{\infty} W\left(1-v_{i} \hat{n}^{i}\right) \tilde{\kappa}_{a,(\tilde{\nu})} I_{(\nu)} \mathrm{d} \nu}{\int_{0}^{\infty} I_{(\nu)} \mathrm{d} \nu}=W\left(1-v_{i} \hat{n}^{i}\right) \tilde{\kappa}_{a} .
$$

The frequency-integrated emissivity [see Eq. (2.92)] in the lab frame follows as

$$
\eta=\int_{0}^{\infty} \nu^{3} \eta_{(\nu)} \mathrm{d} \nu=\frac{\int_{0}^{\infty} \tilde{\nu}^{3} \tilde{\eta}_{(\tilde{\nu})} \mathrm{d} \nu}{W^{2}\left(1-v_{i} \hat{n}^{i}\right)^{2}}=\frac{\int_{0}^{\infty} \tilde{\nu}^{3} \tilde{\eta}_{(\tilde{\nu})} \mathrm{d} \tilde{\nu}}{W^{3}\left(1-v_{i} \hat{n}^{i}\right)^{3}}=\frac{\tilde{\eta}}{W^{3}\left(1-v_{i} \hat{n}^{i}\right)^{3}}
$$

The transformation of the scattering opacities is more complicated. We here only consider iso-energetic isotropic scattering (see Sec. 5.2.4, where we use Thomson scattering). From Eq. (5.102) we can see that there are two terms through which $\kappa_{0}$ enters. One is proportional to the specific intensity and thus acts like an absorption 
term. Accordingly it transforms like $\kappa_{a}$ in Eq. (B.4), i.e.,

$$
\kappa_{0} I=W\left(1-v_{i} \hat{n}^{i}\right) \tilde{\kappa}_{0} I .
$$

The second term proportional to $E$ acts like an emission term and transforms accordingly as

$$
\kappa_{0} E=\frac{\tilde{\kappa}_{0} J}{W^{3}\left(1-v_{i} \hat{n}^{i}\right)^{3}},
$$

where we recall $J$ being the radiation energy density in the fluid frame [see Eq. (5.129)].

Replacing all the lab-frame opacities as well as the lab-frame emissivity in Eq. (5.102) results in the mixed-frame equation

$$
\left(\frac{\partial}{\partial t}+\hat{\boldsymbol{n}} \cdot \boldsymbol{\nabla}\right) I=-W\left(1-v_{i} \hat{n}^{i}\right)\left(\tilde{\kappa}_{a}+\tilde{\kappa}_{0}\right) I+\frac{\tilde{\eta}+\tilde{\kappa}_{0} J}{W^{3}\left(1-v_{i} \hat{n}^{i}\right)^{3}} .
$$

This is the equation that we solve for the simulation of the relativistic jet in Sec. 5.2.4. 


\section{Link Between Microscopic and Macroscopic Parameters}

The scattering coefficients $\kappa_{0}$ and $\kappa_{1}$ in the RTE equation (5.102) describe the microscopic interactions with the fluid. In this appendix is shown how these microscopic quantities are linked to the macroscopic ones within the LB method. Specifically, we perform an asymptotic analysis in the limit of zero absorption and emissivity. In this way we show that the radiative LB equation (5.109) is a solution to the macroscopic diffusion equation

$$
\partial_{t} E=D \Delta E
$$

with diffusion coefficient $D$.

We first establish the link between $D$ and $\left(\kappa_{0}, \kappa_{1}\right)$ via a Chapman-Enskog expansion (Chapman \& Cowling, 1970) in Sec. C.1. Then, the analytic expressions are extended in Sec. C.2 via a fit derived from numerical simulations in order to also account for numerical corrections.

\section{C.1 Chapman-Enskog Analysis}

For zero emission and absorption, the radiative LB equation reads

$$
I_{(i)}\left(\boldsymbol{r}+\hat{\boldsymbol{n}}_{(i)} \Delta t, t+\Delta t\right)-I_{(i)}(\boldsymbol{r}, t)=-\kappa_{0}\left(I_{(i)}(\boldsymbol{r}, t)-I_{(i)}^{\mathrm{eq}}(\boldsymbol{r}, t)\right) .
$$

A Taylor expansion of the LHS of this equation yields up to second order

$$
\begin{aligned}
& I_{(i)}\left(\boldsymbol{r}+\hat{\boldsymbol{n}}_{(i)} \Delta t, t+\Delta t\right)-I_{(i)}(\boldsymbol{r}, t)= \\
& \quad \Delta t\left(\partial_{t}+\hat{n}_{(i)}^{\mu} \partial_{\mu}\right) I_{(i)}(\boldsymbol{r}, t)+\frac{1}{2} \Delta t^{2}\left(\partial_{t}+\hat{n}_{(i)}^{\mu} \partial_{\mu}\right)^{2} I_{(i)}(\boldsymbol{r}, t)+\mathcal{O}\left(\Delta t^{3}\right),
\end{aligned}
$$

where we have written vector components with Greek indices.

In order to see how this expansion becomes the macroscopic diffusion equation, we first need to collect a number of equations. We start this collection by expanding the differential operators and the specific intensities as follows:

$$
\begin{aligned}
\partial_{t} & =\epsilon \partial_{t}^{(1)}+\epsilon^{2} \partial_{t}^{(2)}+\mathcal{O}\left(\epsilon^{3}\right) \\
\partial_{\mu} & =\epsilon \partial_{\mu}^{(1)}+\mathcal{O}\left(\epsilon^{2}\right) \\
I_{(i)} & \left.=I_{(i)}^{(0)}+\epsilon I_{(i)}^{(1)}+\epsilon^{2} I_{(i)}^{(2)}+\right)\left(\epsilon^{3}\right),
\end{aligned}
$$

where $\epsilon \ll 1$ and $I_{(i)}^{(0)} \equiv I_{(i)}^{\mathrm{eq}}$. 
For the numerical moments of the equilibrium specific intensity we find

$$
\begin{aligned}
\sum_{i} I_{(i)}^{\mathrm{eq}} & =E \\
\sum_{i} I_{(i)}^{\mathrm{eq}} \hat{n}_{(i)}^{\mu} & =\frac{\lambda}{d} F^{\mu}, \\
\sum_{i} I_{(i)}^{\mathrm{eq}} \hat{n}_{(i)}^{\mu} \hat{n}_{(i)}^{\nu} & =\frac{1}{d} \delta_{\mu \nu} E,
\end{aligned}
$$

where we used the $d$-dimensional isotropy conditions

$$
\begin{array}{cl}
\sum_{i} w_{(i)} & =1, \quad \sum_{i} w_{(i)} \hat{n}_{(i)}^{\mu}=0, \\
\sum_{i} w_{(i)} \hat{n}_{(i)}^{\mu} \hat{n}_{(i)}^{\nu}=\frac{1}{d} \delta_{\mu \nu}, & \sum_{i} w_{(i)} \hat{n}_{(i)}^{\mu} \hat{n}_{(i)}^{\nu} \hat{n}_{(i)}^{\rho}=0 .
\end{array}
$$

Further, we know that in the diffusion limit the radiation is in thermodynamic equilibrium with the fluid background. Without absorption and emission we can thus write

$$
\int_{4 \pi}\left(I-I^{\mathrm{eq}}\right) \mathrm{d} \Omega=0
$$

from which follows

$$
\int_{4 \pi} I \mathrm{~d} \Omega=\int_{4 \pi} I^{\mathrm{eq}} \mathrm{d} \Omega
$$

For the discrete LB equation we then have the conditions

$$
\begin{aligned}
& \sum_{i} I_{(i)}=\sum_{i} I_{(i)}^{\mathrm{eq}}=E, \\
& \sum_{i} I_{(i)}^{(k)}=0 \quad \forall k \geq 1 .
\end{aligned}
$$

We now have all the ingredients to complete the expansion. Inserting Eq. (C.3) into the RTE yields

$$
\Delta t\left(\partial_{t}+\hat{n}_{(i)}^{\mu} \partial_{\mu}\right) I_{(i)}(\boldsymbol{r}, t)+\frac{1}{2} \Delta t^{2}\left(\partial_{t}+\hat{n}_{(i)}^{\mu} \partial_{\mu}\right)^{2} I_{(i)}(\boldsymbol{r}, t)=-\kappa_{0}\left(I_{(i)}(\boldsymbol{r}, t)-I_{(i)}^{\mathrm{eq}}(\boldsymbol{r}, t)\right) .
$$

Via inserting Eqs. (C.4)-(C.6) we obtain a multi-scale expansion. Collection all terms of order $\epsilon$ and $\epsilon^{2}$ results in

$$
\begin{aligned}
\mathcal{O}(\epsilon): & \left(\partial_{t}^{(1)}+\hat{n}_{(i)}^{\mu} \partial_{\mu}^{(1)}\right) I_{(i)}^{(0)} \approx-\kappa_{0} I_{(i)}^{(1)} \\
\mathcal{O}\left(\epsilon^{2}\right): & \left(1-\frac{\Delta t}{2} \kappa_{0}\right)\left(\partial_{t}^{(1)}+\hat{n}_{(i)}^{\mu} \partial_{\mu}^{(1)}\right) I_{(i)}^{(1)}+\partial_{t}^{(2)} I_{(i)}^{(0)} \approx \kappa_{0} I_{(i)}^{(2)} .
\end{aligned}
$$

Summing the first of these equations over all populations and using the conservation law Eq. (C.15) together with the definition of the moments yields

$$
\partial_{t}^{(1)} E+\frac{\lambda}{d} \partial_{\mu}^{(1)} F^{\mu}=0
$$


Likewise from Eq. (C.18) we obtain after multiplying by $\hat{\boldsymbol{n}}_{(i)}$ and summing over all populations

$$
\frac{\lambda}{d} \partial_{t}^{(1)} F^{\mu}+\frac{1}{d} \partial_{\mu} E=-\kappa_{0} \sum_{i} \hat{n}_{(i)}^{\mu} I_{(i)}^{(1)} .
$$

Integrating also the second-order equation (C.19), we find

$$
\partial_{t}^{(2)} E=-\left(1-\frac{\Delta t}{2} \kappa_{0}\right) \partial_{\mu}^{(1)} \sum_{i} \hat{n}_{(i)}^{\mu} I_{(i)}^{(1)} .
$$

Inserting Eq. (C.21) into the RHS of this equation and rearranging terms finally yields the desired diffusion equation

$$
\partial_{t}^{(2)} E=D \Delta^{(1)} E+C,
$$

where the diffusion coefficient is linked to the microscopic parameters via

$$
D=\frac{1}{d \kappa_{0}}\left(1-\frac{\Delta t}{2} \kappa_{0}\right)
$$

and the correction term is

$$
C=D \lambda \partial_{\mu}^{(1)} \partial_{t}^{(1)} F^{\mu} .
$$

Note that in three dimensions $(d=3)$ the diffusion coefficient takes the well-known value $D=1 /\left(3 \kappa_{0}\right)$ with an error coming from the time discretization $\Delta t$. Note also that the equations of the M1 system, i.e., Eqs. (5.16) and (5.17) in the absence of absorption and emission and in the limit of high optical depth result in the same diffusion equation with the same correction term as Eq. (C.25). Considering that M1 consists of a set of macroscopic equations, while the LB equation is a microscopic one, it is remarkable the both result in the same macroscopic behavior.

\section{C.2 Numerical Fit for Scattering Opacities}

The Chapman-Enskog analysis of the previous section provides a link between the macroscopic and microscopic parameters. However, we have neglected higher order terms in the final derivation of the diffusion coefficient. Furthermore, we also expect some additional dissipative effects due to the spatial discretization and the interpolation used for populations that do not fall on our Cartesian grid. Instead of deriving all these corrections analytically, we simply perform a numerical fit in this section.

First, we take into consideration the parameter $\lambda$ introduced in Eq. (5.104) and which is not present in the diffusion coefficient, Eq. (C.24). Performing the same diffusion-wave test as in Sec. 5.2.3 and varying the value for $\lambda$ shows that the diffusion speed indeed depends on this parameter. This is also shown in Fig. C.1. We take this into account via a linear dependence, i.e., via the additional term

$$
\chi=\kappa_{0}\left(1+\alpha_{1} \frac{\lambda}{d}\right) .
$$

Additionally, we also take into account the artificial diffusion from the interpolation scheme. In total, we then propose the following extension to Eq. (C.24)

$$
D=\frac{1}{d \chi}\left[1-\left(\frac{1}{2}+\alpha_{2}\right) \Delta t \chi\right]+\frac{\Delta x^{2}}{\Delta t} \alpha_{3},
$$




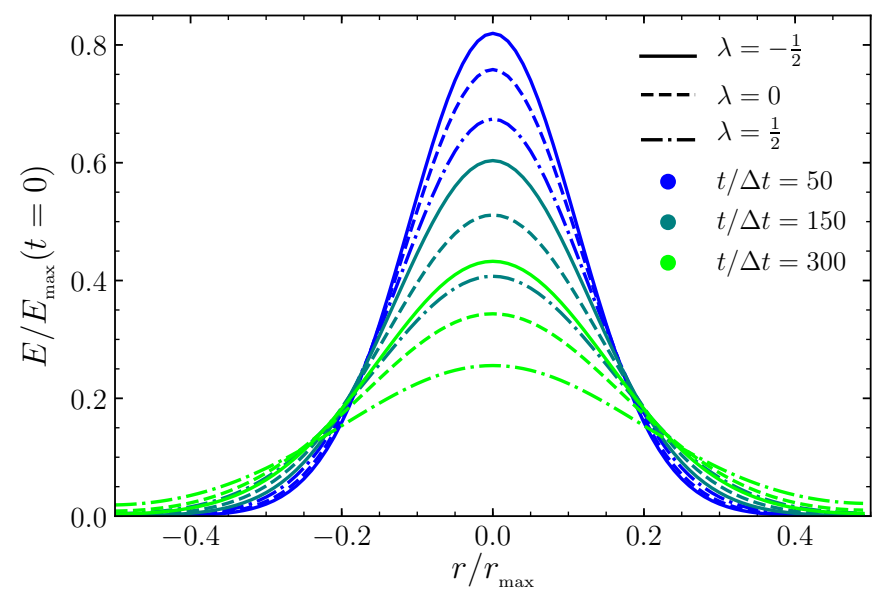

Figure C.1: Impact of the choice of $\lambda$ on the diffusion rate of a scattering wave for fixed $\kappa_{0} \Delta x=1$. Shown are three different values of $\lambda$, each at three different times during the simulation. Reprint of Fig. A1 in Weih et al. (2020a).

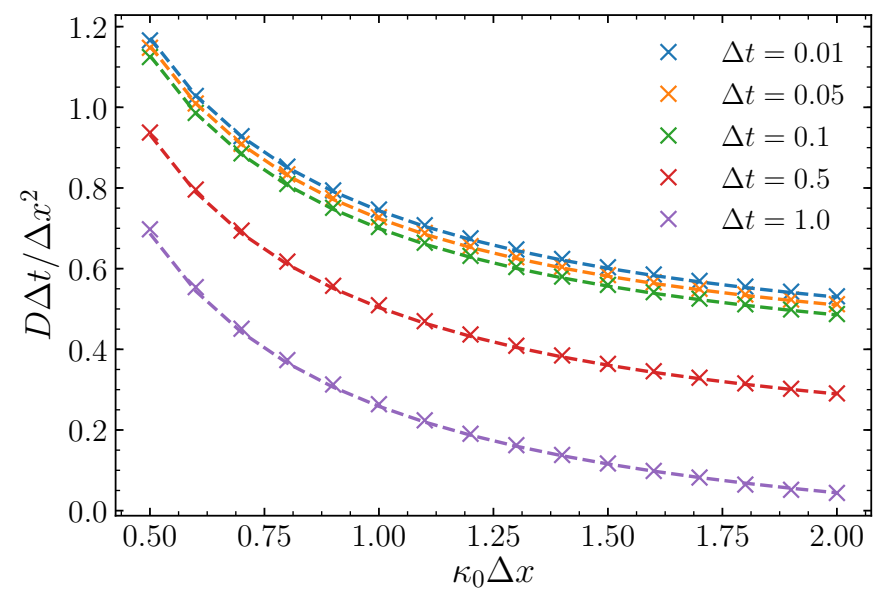

Figure C.2: Example of the numerical procedure for fitting the coefficients $\alpha_{1}$, $\alpha_{2}$, and $\alpha_{3}$ in Eq. (C.27). The crosses show values for the diffusion rate that are estimated by requiring the numerical simulation results to match the analytic ones within a given error range (the L2-norm of the relative error is smaller than 1\%). The dashed lines show the fitted diffusion coefficient, Eq. (C.27). Reprint of Fig. A2 in Weih et al. (2020a).

where $\alpha_{1}, \alpha_{2}$, and $\alpha_{3}$ are parameters of order unity that need to be determined. Note that when explicitly writing the speed of light in this equation, one will find the $\alpha_{2}$ term to show a linear dependence on it. This linear dependence on the propagation speed is also present in other off-lattice LB schemes (Coelho et al., 2018). Also, these additional corrections vanish for $\Delta t$ and $\Delta x$ going to zero.

For obtaining the missing parameters, $\alpha_{i}$, we again perform the diffusion-wave test and vary $\kappa_{0} \Delta x, \Delta t$, and $\lambda$. We then estimate the diffusion coefficient from each simulation by comparing to the analytic solutions [see Eq. (5.127)]. Fig. C.2 shows this exemplary for $\lambda=0.25$. In this way we can fit the missing parameters. We find $\alpha_{1} \approx-4 / 3$, which is a robust value in two and three dimensions. The other parameters depend on the stencil and dimensionality, but as expected seem to converge for large $N_{\text {pop. }}$. To give an example, we obtain $\alpha_{2} \approx 0.48$ and $\alpha_{3} \approx 0.65$ in 2 D with $N_{\text {pop }}=120$. In $3 \mathrm{D}$ we find $\alpha_{2} \approx 0.58$ and $\alpha_{3} \approx 0.77$ for a spherical-design stencil with $N_{\text {pop }}=222$. 


\section{Bibliography}

Abbott, B. P., \& et al., Exploring the sensitivity of next generation gravitational wave detectors, Classical and Quantum Gravity, 34, 044001, arXiv:1607.08697 [astroph.IM], 2017. (Cited on pages 72, 128, and 175.)

Abbott, B. P., Abbott, R., Abbott, T. D., et al., Observation of Gravitational Waves from a Binary Black Hole Merger, Phys. Rev. Lett., 116, 061102, arXiv:1602.03837 [gr-qc], 2016. (Cited on page 1.)

Abbott, B. P., Abbott, R., Abbott, T. D., et al., Gw17081\%: Observation of gravitational waves from a binary neutron star inspiral, Phys. Rev. Lett., 119, 161101, arXiv:1710.05832 [gr-qc], 2017. (Cited on pages 5, 44, 48, 172, and 173.)

Abbott, B. P., Abbott, R., Abbott, T. D., et al., GW170817: Measurements of Neutron Star Radii and Equation of State, Physical Review Letters, 121, 161101, arXiv:1805.11581 [gr-qc], 2018. (Cited on pages 50, 51, and 59.)

Abbott, B. P., Abbott, R., Abbott, T. D., et al., GW190425: Observation of a Compact Binary Coalescence with Total Mass $3.4 M_{\odot}$, Astrophys. J. Lett., 892, L3, arXiv:2001.01761 [astro-ph.HE], 2020. (Cited on pages 58 and 129.)

Abbott, R., Abbott, T. D., Abraham, S., et al., GWTC-2: Compact Binary Coalescences Observed by LIGO and Virgo During the First Half of the Third Observing Run, arXiv e-prints, arXiv:2010.14527, arXiv:2010.14527 [gr-qc], 2020. (Cited on pages 129 and 177.)

Adler, R., Bazin, M., \& Schiffer, M., Introduction to general relativity., 1975. (Cited on page 11.)

Ahrens, C., \& Beylkin, G., Rotationally invariant quadratures for the sphere, Proceedings of the Royal Society A: Mathematical, Physical and Engineering Sciences, 465, 3103, 2009. (Cited on page 110.)

Aker, M., Altenmüller, K., Arenz, M., et al., Improved Upper Limit on the Neutrino Mass from a Direct Kinematic Method by KATRIN, Phys. Rev. Lett., 123, 221802, arXiv:1909.06048 [hep-ex], 2019. (Cited on page 26.)

Akmal, A., Pandharipande, V. R., \& Ravenhall, D. G., Equation of state of nucleon matter and neutron star structure, Phys. Rev. C, 58, 1804, arXiv:nucl-th/9804027 [nucl-th], 1998. (Cited on page 47.)

Alcubierre, M., Introduction to $3+1$ Numerical Relativity (Oxford, UK: Oxford University Press), 2008. (Cited on pages 10, 15, and 19.)

Alcubierre, M., Brügmann, B., Diener, P., et al., Gauge conditions for long-term numerical black hole evolutions without excision, Phys. Rev. D, 67, 084023, arXiv:grqc/0206072, 2003. (Cited on page 19.)

Alford, M., \& Sedrakian, A., Compact Stars with Sequential QCD Phase Transitions, Physical Review Letters, 119, 161104, arXiv:1706.01592 [astro-ph.HE], 2017. (Cited on page 3.) 
Alford, M. G., Bovard, L., Hanauske, M., Rezzolla, L., \& Schwenzer, K., Viscous Dissipation and Heat Conduction in Binary Neutron-Star Mergers, Phys. Rev. Lett., 120, 041101, arXiv:1707.09475 [gr-qc], 2018. (Cited on page 64.)

Alford, M. G., Han, S., \& Prakash, M., Generic conditions for stable hybrid stars, Phys. Rev. D, 88, 083013, arXiv:1302.4732 [astro-ph.SR], 2013. (Cited on page 38.)

Alford, M. G., \& Harris, S. P., $\beta$ equilibrium in neutron-star mergers, Phys. Rev. C, 98, 065806, arXiv:1803.00662 [nucl-th], 2018. (Cited on page 38.)

Alic, D., Bona-Casas, C., Bona, C., Rezzolla, L., \& Palenzuela, C., Conformal and covariant formulation of the Z4 system with constraint-violation damping, Phys. Rev. D, 85, 064040, arXiv:1106.2254 [gr-qc], 2012. (Cited on pages 16 and 17.)

Alic, D., Kastaun, W., \& Rezzolla, L., Constraint damping of the conformal and covariant formulation of the Z4 system in simulations of binary neutron stars, Phys. Rev. D, 88, 064049, arXiv:1307.7391 [gr-qc], 2013. (Cited on page 17.)

Alic, D., Moesta, P., Rezzolla, L., Zanotti, O., \& Jaramillo, J. L., Accurate Simulations of Binary Black Hole Mergers in Force-free Electrodynamics, Astrophys. J., 754, 36, arXiv:1204.2226 [gr-qc], 2012. (Cited on pages 82 and 85.)

Aloy, M. A., Ibáñez, J. M. ., Martí, J. M. ., Gómez, J.-L., \& Müller, E., HighResolution Three-dimensional Simulations of Relativistic Jets, Astrophys. J., 523, L125, arXiv:arXiv:astro-ph/9906428, 1999. (Cited on pages 123 and 124.)

Aloy, M. A., \& Rezzolla, L., A powerful hydrodynamic booster for relativistic jets, Astrophys. J., 640, L115, 2006. (Cited on page 124.)

Alvarez-Castillo, D., Ayriyan, A., Benic, S., et al., New class of hybrid EoS and Bayesian $M-R$ data analysis, European Physical Journal A, 52, 69, arXiv:1603.03457 [nucl-th], 2016. (Cited on page 61.)

Alvarez-Castillo, D. E., Blaschke, D. B., Grunfeld, A. G., \& Pagura, V. P., Third family of compact stars within a nonlocal chiral quark model equation of state, Phys. Rev. D, 99, 063010, arXiv:1805.04105 [hep-ph], 2019. (Cited on pages 2, 58, and 61.)

Ambruş, V. E., \& Blaga, R., High-order quadrature-based lattice boltzmann models for the flow of ultrarelativistic rarefied gases, Phys. Rev. C, 98, 035201, 2018. (Cited on page 108.)

Annala, E., Ecker, C., Hoyos, C., et al., Holographic compact stars meet gravitational wave constraints, Journal of High Energy Physics, 2018, 78, arXiv:1711.06244 [astro-ph.HE], 2018. (Cited on page 3.)

Annala, E., Gorda, T., Kurkela, A., Nättilä, J., \& Vuorinen, A., Evidence for quarkmatter cores in massive neutron stars, Nature Physics, 16, 907, 2020. (Cited on pages 2,58 , and 61 .)

Annala, E., Gorda, T., Kurkela, A., \& Vuorinen, A., Gravitational-Wave Constraints on the Neutron-Star-Matter Equation of State, Phys. Rev. Lett., 120, 172703, arXiv:1711.02644 [astro-ph.HE], 2018. (Cited on pages 38, 39, 48, and 50.)

Anninos, P., \& Fragile, P. C., Multi-frequency General Relativistic Radiationhydrodynamics with $M_{1}$ Closure, Astrophys. J., 900, 71, arXiv:2007.12195 [astroph.IM], 2020. (Cited on pages 30, 76, and 102.) 
Ansorg, M., Brügmann, B., \& Tichy, W., A single-domain spectral method for black hole puncture data, Phys. Rev. D, 70, 064011, arXiv:gr-qc/0404056, 2004. (Cited on page 15.)

Ansorg, M., Gondek-Rosińska, D., \& Villain, L., On the solution space of differentially rotating neutron stars in general relativity, Mon. Not. R. Astron. Soc., 396, 2359, arXiv:0812.3347 [gr-qc], 2009. (Cited on page 44.)

Antoniadis, J., Freire, P. C. C., Wex, N., et al., A Massive Pulsar in a Compact Relativistic Binary, Science, 340, 448, arXiv:1304.6875 [astro-ph.HE], 2013. (Cited on pages $2,38,49,55$, and 173 .)

Arnowitt, R., Deser, S., \& Misner, C. W., Republication of: The dynamics of general relativity, General Relativity and Gravitation, 40, 1997, arXiv:gr-qc/0405109, 2008. (Cited on page 15.)

Arzoumanian, Z., Gendreau, K. C., Baker, C. L., et al., The neutron star interior composition explorer (NICER): mission definition, in Proceedings of the SPIE, Vol. 9144, Space Telescopes and Instrumentation 2014: Ultraviolet to Gamma Ray 914420, 2014. (Cited on pages 51 and 53.)

Asinari, P., Mishra, S., \& Borchiellini, R., A lattice boltzmann formulation for the analysis of radiative heat transfer problems in a participating medium, Numerical Heat Transfer, Part B: Fundamentals, 57, 126, 2010. (Cited on page 105.)

Atkinson, K., \& Han, W., Spherical harmonics and approximations on the unit sphere: An introduction (Springer Berlin Heidelberg), 2012. (Cited on page 108.)

Audit, E., Charrier, P., Chièze, J. P., \& Dubroca, B., A radiation-hydrodynamics scheme valid from the transport to the diffusion limit, arXiv e-prints, astro, arXiv:astro-ph/0206281 [astro-ph], 2002. (Cited on page 82.)

Ayriyan, A., Bastian, N.-U., Blaschke, D., et al., Robustness of third family solutions for hybrid stars against mixed phase effects, Physical Review C, 97, 045802, arXiv:1711.03926 [nucl-th], 2018. (Cited on page 61.)

Baiotti, L., Giacomazzo, B., \& Rezzolla, L., Accurate evolutions of inspiralling neutron-star binaries: Prompt and delayed collapse to a black hole, Phys. Rev. D, 78, 084033, arXiv:0804.0594 [gr-qc], 2008. (Cited on page 64.)

Baiotti, L., \& Rezzolla, L., Binary neutron-star mergers: a review of Einstein's richest laboratory, Rept. Prog. Phys., 80, 096901, arXiv:1607.03540 [gr-qc], 2017. (Cited on pages $2,3,62$, and 127 .)

Banyuls, F., Font, J. A., Ibáñez, J. M., Martí, J. M., \& Miralles, J. A., Numerical 3+1 general-relativistic hydrodynamics: A local characteristic approach, Astrophys. J., 476, 221, 1997. (Cited on pages 22 and 80.)

Barnes, J., Kasen, D., Wu, M.-R., \& Martínez-Pinedo, G., Radioactivity and Thermalization in the Ejecta of Compact Object Mergers and Their Impact on Kilonova Light Curves, Astrophys. J., 829, 110, arXiv:1605.07218 [astro-ph.HE], 2016. (Cited on page 45.)

Barsotti, L., Fritschel, P., Evans, M., \& Gras, S., Ligo document t1800044-v5, https://dcc.ligo.org/LIGO-T1800044/public, 2020. (Cited on pages 71 and 128.) 
Baumgarte, T. W., \& Shapiro, S. L., Numerical integration of Einstein's field equations, Phys. Rev. D, 59, 024007, arXiv:gr-qc/9810065, 1999. (Cited on page 15.)

Baumgarte, T. W., \& Shapiro, S. L., General Relativistic Magnetohydrodynamics for the Numerical Construction of Dynamical Spacetimes, Astrophys. Journal, 585, 921, arXiv:arXiv:astro-ph/0211340, 2003. (Cited on page 23.)

Baumgarte, T. W., \& Shapiro, S. L., Numerical Relativity: Solving Einstein's Equations on the Computer (Cambridge, UK: Cambridge University Press), 2010. (Cited on pages 10,13, 19, 20, and 21.)

Bauswein, A., Bastian, N.-U. F., Blaschke, D. B., et al., Identifying a First-Order Phase Transition in Neutron-Star Mergers through Gravitational Waves, Physical Review Letters, 122, 061102, arXiv:1809.01116 [astro-ph.HE], 2019. (Cited on pages $60,62,66,73,127,128$, and 174 .)

Bauswein, A., Baumgarte, T. W., \& Janka, H.-T., Prompt Merger Collapse and the Maximum Mass of Neutron Stars, Phys. Rev. Lett., 111, 131101, arXiv:1307.5191 [astro-ph.SR], 2013. (Cited on pages 3, 45, 50, and 51.)

Bauswein, A., \& Blacker, S., Impact of quark deconfinement in neutron star mergers and hybrid star mergers, arXiv e-prints, arXiv:2006.16183, arXiv:2006.16183 [astroph.HE], 2020. (Cited on pages 73 and 128.)

Bauswein, A., Janka, H., \& Oechslin, R., Testing approximations of thermal effects in neutron star merger simulations, Phys. Rev. D, 82, 084043, arXiv:1006.3315 [astro-ph.SR], 2010. (Cited on page 64.)

Bauswein, A., \& Janka, H.-T., Measuring Neutron-Star Properties via Gravitational Waves from Neutron-Star Mergers, Phys. Rev. Lett., 108, 011101, arXiv:1106.1616 [astro-ph.SR], 2012. (Cited on pages 5 and 70.)

Bauswein, A., \& Janka, H.-T., -, Phys. Rev. Lett., 108, 011101, arXiv:1106.1616 [astro-ph.SR], 2012. (Cited on pages 62 and 174.)

Bauswein, A., Just, O., Janka, H.-T., \& Stergioulas, N., Neutron-star Radius Constraints from GW170817 and Future Detections, Astrophys. J. Lett., 850, L34, arXiv:1710.06843 [astro-ph.HE], 2017. (Cited on page 51.)

Baym, G., Pethick, C., \& Sutherland, P., The Ground State of Matter at High Densities: Equation of State and Stellar Models, Astrophys. J., 170, 299, 1971. (Cited on page 38.)

Beentjes, C. H. L., Quadrature on a Spherical Surface, International Conference on Mathematics and Computational Methods Applied to Nuclear Science and Engineering, 1, 2015. (Cited on page 110.)

Begelman, M. C., Black holes in radiation-dominated gas: an analogue of the Bondi accretion problem., Mon. Not. R. Astron. Soc., 184, 53, 1978. (Cited on page 94.)

Benzi, R., Succi, S., \& Vergassola, M., The lattice Boltzmann equation: theory and applications, Phys. Rep., 222, 145, 1992. (Cited on page 102.)

Berger, E., Short-Duration Gamma-Ray Bursts, Annual Review of Astron. and Astrophys., 52, 43, arXiv:1311.2603 [astro-ph.HE], 2014. (Cited on page 45.) 
Bernaschi, M., Fatica, M., Melchionna, S., Succi, S., \& Kaxiras, E., A flexible highperformance lattice boltzmann gpu code for the simulations of fluid flows in complex geometries, Concurrency and Computation: Practice and Experience, 22, 1, 2010. (Cited on pages 120 and 121.)

Bernaschi, M., Melchionna, S., Succi, S., et al., Muphy: A parallel multi physics/scale code for high performance bio-fluidic simulations, Computer Physics Communications, 180, 1495, 2009. (Cited on page 120.)

Bernuzzi, S., Dietrich, T., \& Nagar, A., Modeling the Complete Gravitational Wave Spectrum of Neutron Star Mergers, Phys. Rev. Lett., 115, 091101, arXiv:1504.01764 [gr-qc], 2015. (Cited on pages 5, 62, 70, and 175.)

Bernuzzi, S., \& Hilditch, D., Constraint violation in free evolution schemes: comparing BSSNOK with a conformal decomposition of Z4, Phys. Rev. D, 81, 084003, arXiv:0912.2920 [gr-qc], 2010. (Cited on page 17.)

Bethe, H. A., \& Wilson, J. R., Revival of a stalled supernova shock by neutrino heating, Astrophys. J., 295, 14, 1985. (Cited on page 2.)

Bezares, M., Palenzuela, C., \& Bona, C., Final fate of compact boson star mergers, Phys. Rev. D, 95, 124005, arXiv:1705.01071 [gr-qc], 2017. (Cited on page 18.)

Bhatnagar, P. L., Gross, E. P., \& Krook, M., A model for collision processes in gases. i. small amplitude processes in charged and neutral one-component systems, Phys. Rev., 94, 511, 1954. (Cited on page 104.)

Bilous, A. V., Watts, A. L., Harding, A. K., et al., A NICER View of PSR J0030+0451: Evidence for a Global-scale Multipolar Magnetic Field, Astrophys. J. Lett., 887, L23, arXiv:1912.05704 [astro-ph.HE], 2019. (Cited on pages 57, 58, and 173.)

Bindra, H., \& Patil, D. V., Radiative or neutron transport modeling using a lattice boltzmann equation framework, Phys. Rev. E, 86, 016706, 2012. (Cited on page 105.)

Birkhoff, G. D., Relativity and modern physics (Cambridge, Mass., U.S.A.: Harvard University Press), 1923. (Cited on page 37.)

Blacker, S., Bastian, N.-U. F., Bauswein, A., et al., Constraining the onset density of the hadron-quark phase transition with gravitational-wave observations, arXiv e-prints, arXiv:2006.03789, arXiv:2006.03789 [astro-ph.HE], 2020. (Cited on pages 73 and 128.)

Bogdanov, S., Guillot, S., Ray, P. S., et al., Constraining the Neutron Star MassRadius Relation and Dense Matter Equation of State with NICER. I. The Millisecond Pulsar X-Ray Data Set, Astrophys. J. Lett., 887, L25, arXiv:1912.05706 [astro-ph.HE], 2019. (Cited on pages 3, 57, and 173.)

Bogdanov, S., Lamb, F. K., Mahmoodifar, S., et al., Constraining the Neutron Star Mass-Radius Relation and Dense Matter Equation of State with NICER. II. Emission from Hot Spots on a Rapidly Rotating Neutron Star, Astrophys. J. Lett., 887, L26, arXiv:1912.05707 [astro-ph.HE], 2019. (Cited on pages 3, 57, and 173.)

Bombaci, I., The maximum mass of a neutron star., Astron. Astrophys., 305, 871, 1996. (Cited on page 37.) 
Bona, C., Ledvinka, T., Palenzuela, C., \& Zácek, M., General-covariant evolution formalism for numerical relativity, Phys. Rev. D, 67, 104005, arXiv:gr-qc/0302083, 2003. (Cited on page 16.)

Bona, C., Massó, J., Seidel, E., \& Stela, J., First order hyperbolic formalism for numerical relativity, Phys. Rev. D, 56, 3405, arXiv:gr-qc/9709016, 1997. (Cited on page 19.)

Bondi, H., On spherically symmetric accretion, Mon. Not. R. Astron. Soc., 112, 195, 1952. (Cited on page 94.)

Bovard, L., Martin, D., Guercilena, F., et al., On r-process nucleosynthesis from matter ejected in binary neutron star mergers, Phys. Rev. D, 96, 124005, arXiv:1709.09630 [gr-qc], 2017. (Cited on pages 5 and 45.)

Bozzola, G., Espino, P. L., Davis Lewin, C., \& Paschalidis, V., Maximum mass and universal relations of rotating relativistic hybrid hadron-quark stars, arXiv e-prints, arXiv:1905.00028, arXiv:1905.00028 [astro-ph.HE], 2019. (Cited on page 47.)

Breu, C., \& Rezzolla, L., Maximum mass, moment of inertia and compactness of relativistic stars, Mon. Not. R. Astron. Soc., 459, 646, arXiv:1601.06083 [gr-qc], 2016. (Cited on pages 44, 47, and 172.)

Brown, D., Diener, P., Sarbach, O., Schnetter, E., \& Tiglio, M., Turduckening black holes: an analytical and computational study, Phys. Rev. D, 79, 044023, arXiv:arXiv:0809.3533 [gr-qc], 2009. (Cited on page 65.)

Bruenn, S. W., Stellar core collapse - Numerical model and infall epoch, Astrophys. J., Supp., 58, 771, 1985. (Cited on pages 27, 78, 106, and 126.)

Bruenn, S. W., Mezzacappa, A., \& Dineva, T., Dynamic and diffusive instabilities in core collapse supernovae., Physics Reports, 256, 69, 1995. (Cited on page 31.)

Brügmann, B., González, J. A., Hannam, M., et al., Calibration of moving puncture simulations, Phys. Rev. D, 77, 024027, arXiv:gr-qc/0610128, 2008. (Cited on pages 19 and 20.)

Bucciantini, N., Metzger, B. D., Thompson, T. A., \& Quataert, E., Short gamma-ray bursts with extended emission from magnetar birth: jet formation and collimation, Mon. Not. R. Astron. Soc., 419, 1537, arXiv:1106.4668 [astro-ph.HE], 2012. (Cited on page 45.$)$

Burgio, G. F., Drago, A., Pagliara, G., Schulze, H.-J., \& Wei, J.-B., Are Small Radii of Compact Stars Ruled out by GW170817/AT2017gfo?, Astrophys. J., 860, 139, 2018. (Cited on page 50.)

Burrows, A., Reddy, S., \& Thompson, T. A., Neutrino opacities in nuclear matter, Nuclear Physics A, 777, 356, arXiv:astro-ph/0404432, 2006. (Cited on page 125.)

Calore, E., Gabbana, A., Kraus, J., Schifano, S. F., \& Tripiccione, R., Performance and portability of accelerated lattice Boltzmann applications with OpenACC, Concurrency and Computation: Practice and Experience, 28, 3485, 2016. (Cited on pages 121 and 128.)

Calore, E., Gabbana, A., Schifano, S., \& Tripiccione, R., Optimization of lattice boltzmann simulations on heterogeneous computers, The International Journal of High Performance Computing Applications, 33, 124, 2019. (Cited on page 120.) 
Capano, C. D., Tews, I., Brown, S. M., et al., Stringent constraints on neutron-star radii from multimessenger observations and nuclear theory, Nature Astronomy, arXiv:1908.10352 [astro-ph.HE], 2020. (Cited on page 38.)

Cardall, C. Y., Endeve, E., \& Mezzacappa, A., Conservative $3+1$ general relativistic Boltzmann equation, Phys. Rev. D, 88, 023011, arXiv:1305.0037 [astro-ph.HE], 2013. (Cited on pages 28, 77, and 126.)

Carroll, B., \& Ostlie, D., An introduction to modern astrophysics (Pearson AddisonWesley), 2007. (Cited on page 1.)

Carroll, S. M., Spacetime and geometry. An introduction to general relativity, 2004. (Cited on page 10.)

Chapman, S., \& Cowling, T. G., The mathematical theory of non-uniform gases, 3rd ed (Cambridge University Press), 1970. (Cited on pages 119 and 134.)

Christian, J.-E., \& Schaffner-Bielich, J., Twin Stars and the Stiffness of the Nuclear Equation of State: Ruling Out Strong Phase Transitions below $1.7 n_{0}$ with the New NICER Radius Measurements, Astrophys. J. Lett., 894, L8, arXiv:1912.09809 [astro-ph.HE], 2020. (Cited on page 58.)

Christian, J.-E., Zacchi, A., \& Schaffner-Bielich, J., Classifications of twin star solutions for a constant speed of sound parameterized equation of state, European Physical Journal A, 54, 28, arXiv:1707.07524 [astro-ph.HE], 2018. (Cited on pages 2, 58 , and 64 .)

Christian, J.-E., Zacchi, A., \& Schaffner-Bielich, J., Signals in the tidal deformability for phase transitions in compact stars with constraints from GW170817, Phys. Rev. D, 99, 023009, arXiv:1809.03333 [astro-ph.HE], 2019. (Cited on page 61.)

Ciolfi, R., Kastaun, W., Giacomazzo, B., et al., General relativistic magnetohydrodynamic simulations of binary neutron star mergers forming a long-lived neutron star, Phys. Rev. D, 95, 063016, arXiv:1701.08738 [astro-ph.HE], 2017. (Cited on page 5.)

Coelho, R. C., Mendoza, M., Doria, M. M., \& Herrmann, H. J., Fully dissipative relativistic lattice boltzmann method in two dimensions, Computers \& Fluids, 172, 318 , 2018. (Cited on pages 108 and 137.)

Colgate, S. A., \& White, R. H., The Hydrodynamic Behavior of Supernovae Explosions, Astrophys. J., 143, 626, 1966. (Cited on page 2.)

Collins, J. C., \& Perry, M. J., Superdense matter: Neutrons or asymptotically free quarks?, Phys. Rev. L, 34, 1353, 1975. (Cited on page 2.)

Coulter, D. A., Foley, R. J., Kilpatrick, C. D., et al., Swope Supernova Survey 2017a (SSS17a), the optical counterpart to a gravitational wave source, Science, 358, 1556, arXiv:1710.05452 [astro-ph.HE], 2017. (Cited on page 45.)

Courant, R., \& Hilbert, D., Methods of mathematical physics (New York: John Wiley and Sons, Inc), 1962. (Cited on page 24.)

Cowperthwaite, P. S., Berger, E., Villar, V. A., et al., The Electromagnetic Counterpart of the Binary Neutron Star Merger LIGO/Virgo GW170817. II. UV, Optical, and Near-infrared Light Curves and Comparison to Kilonova Models, Astrophys. 
J. Lett., 848, L17, arXiv:1710.05840 [astro-ph.HE], 2017. (Cited on pages 5, 45, 47, 74, 172,173 , and 175.)

Cromartie, H. T., Fonseca, E., Ransom, S. M., et al., Relativistic Shapiro delay measurements of an extremely massive millisecond pulsar, Nature Astronomy, 4, 72, arXiv:1904.06759 [astro-ph.HE], 2020. (Cited on pages 2, 38, and 59.)

Cutler, C., \& Thorne, K. S., An Overview of Gravitational-Wave Sources, arXiv eprints, gr, arXiv:gr-qc/0204090 [gr-qc], 2002. (Cited on page 21.)

Damour, T., \& Nagar, A., Relativistic tidal properties of neutron stars, Phys. Rev. D, 80, 084035, arXiv:0906.0096 [gr-qc], 2009. (Cited on page 41.)

Daverio, D., Dirian, Y., \& Mitsou, E., Apples with Apples comparison of $3+1$ conformal numerical relativity schemes, arXiv e-prints, arXiv:1810.12346, arXiv:1810.12346 [gr-qc], 2018. (Cited on page 18.)

De, S., Finstad, D., Lattimer, J. M., et al., Tidal Deformabilities and Radii of Neutron Stars from the Observation of GW170817, Physical Review Letters, 121, 091102, arXiv:1804.08583 [astro-ph.HE], 2018. (Cited on pages 2, 50, and 58.)

De Pietri, R., Feo, A., Maione, F., \& Löffler, F., Modeling equal and unequal mass binary neutron star mergers using public codes, Phys. Rev. D, 93, 064047, arXiv:1509.08804 [gr-qc], 2016. (Cited on page 64.)

Deaton, M. B., O'Connor, E., Zhu, Y. L., et al., Elastic scattering in general relativistic ray tracing for neutrinos, Phys. Rev. D, 98, 103014, arXiv:1806.10255 [astro-ph.HE], 2018. (Cited on page 29.)

Del Zanna, L., Zanotti, O., Bucciantini, N., \& Londrillo, P., ECHO: a Eulerian conservative high-order scheme for general relativistic magnetohydrodynamics and magnetodynamics, Astron. Astrophys., 473, 11, arXiv:0704.3206, 2007. (Cited on page 23.)

Delsarte, P., Goethals, J. M., \& Seidel, J. J., Spherical codes and designs, Geometriae Dedicata, 6, 363, 1977. (Cited on page 111.)

Demircik, T., Ecker, C., \& Järvinen, M., Rapidly Spinning Compact Stars with Deconfinement Phase Transition, arXiv e-prints, arXiv:2009.10731, arXiv:2009.10731 [astro-ph.HE], 2020. (Cited on page 44.)

Demorest, P. B., Pennucci, T., Ransom, S. M., Roberts, M. S. E., \& Hessels, J. W. T., A two-solar-mass neutron star measured using Shapiro delay, Nature, 467, 1081, arXiv:1010.5788 [astro-ph.HE], 2010. (Cited on pages 2 and 55.)

Dennison, K. A., Baumgarte, T. W., \& Shapiro, S. L., Maximally rotating supermassive stars at the onset of collapse: effects of gas pressure, Mon. Not. R. Astron. Soc., 488, 4195, arXiv:1906.04190 [astro-ph.HE], 2019. (Cited on page 44.)

Dexheimer, V., Gomes, R. O., Klähn, T., Han, S., \& Salinas, M., GW190814 as a massive rapidly-rotating neutron star with exotic degrees of freedom, arXiv e-prints, arXiv:2007.08493, arXiv:2007.08493 [astro-ph.HE], 2020. (Cited on page 59.)

Dexheimer, V., \& Schramm, S., Proto-Neutron and Neutron Stars in a Chiral SU(3) Model, Astrophys. J., 683, 943, arXiv:0802.1999 [astro-ph], 2008. (Cited on page 62.) 
Dexheimer, V. A., \& Schramm, S., A Novel Approach to Model Hybrid Stars, Phys. Rev., C81, 045201, arXiv:0901.1748 [astro-ph.SR], 2010. (Cited on page 62.)

D'Humières, D., Lallemand, P., \& Frisch, U., Lattice gas models for 3D hydrodynamics, EPL (Europhysics Letters), 2, 291, 1986. (Cited on page 108.)

Dietrich, T., Coughlin, M. W., Pang, P. T. H., et al., New Constraints on the Supranuclear Equation of State and the Hubble Constant from Nuclear Physics - Multi-Messenger Astronomy, arXiv e-prints, arXiv:2002.11355, arXiv:2002.11355 [astro-ph.HE], 2020. (Cited on page 38.)

Dietrich, T., Hinderer, T., \& Samajdar, A., Interpreting Binary Neutron Star Mergers: Describing the Binary Neutron Star Dynamics, Modelling Gravitational Waveforms, and Analyzing Detections, arXiv e-prints, arXiv:2004.02527, arXiv:2004.02527 [gr-qc], 2020. (Cited on pages 3, 60, 62, and 127.)

Dietrich, T., Ujevic, M., Tichy, W., Bernuzzi, S., \& Brügmann, B., Gravitational waves and mass ejecta from binary neutron star mergers: Effect of the mass ratio, Phys. Rev. D, 95, 024029, arXiv:1607.06636 [gr-qc], 2017. (Cited on page 5.)

Dietrich, T., Khan, S., Dudi, R., et al., Matter imprints in waveform models for neutron star binaries: Tidal and self-spin effects, Phys. Rev. D, 99, 024029, arXiv:1804.02235 [gr-qc], 2019. (Cited on pages 5 and 70.)

Dionysopoulou, K., Alic, D., Palenzuela, C., Rezzolla, L., \& Giacomazzo, B., Generalrelativistic resistive magnetohydrodynamics in three dimensions: Formulation and tests, Phys. Rev. D, 88, 044020, arXiv:1208.3487 [gr-qc], 2013. (Cited on pages 82 and 85.)

Douchin, F., \& Haensel, P., A unified equation of state of dense matter and neutron star structure, Astron. Astrophys., 380, 151, arXiv:arXiv:astro-ph/0111092, 2001. (Cited on page 47.)

Drago, A., Lavagno, A., \& Parenti, I., Burning of a Hadronic Star into a Quark or a Hybrid Star, Astrophys. J., 659, 1519, arXiv:arXiv:astro-ph/0512652, 2007. (Cited on page 2.)

Drischler, C., Carbone, A., Hebeler, K., \& Schwenk, A., Neutron matter from chiral two- and three-nucleon calculations up to $N^{3} L O$, Phys. Rev. C, 94, 054307, arXiv:1608.05615 [nucl-th], 2016. (Cited on page 38.)

Drischler, C., Hebeler, K., \& Schwenk, A., Chiral interactions up to $N^{3} L O$ and nuclear saturation, ArXiv e-prints, arXiv:1710.08220 [nucl-th], 2017. (Cited on pages 38 and 39.)

Drout, M. R., Piro, A. L., Shappee, B. J., et al., Light curves of the neutron star merger GW170817/SSS17a: Implications for r-process nucleosynthesis, Science, 358, 1570, arXiv:1710.05443 [astro-ph.HE], 2017. (Cited on pages 5, 45, 47, 74, 172, 173 , and 175.)

Ecker, C., Järvinen, M., Nijs, G., \& van der Schee, W., Gravitational waves from holographic neutron star mergers, Phys. Rev. D, 101, 103006, arXiv:1908.03213 [astro-ph.HE], 2020. (Cited on page 3.)

Eichler, D., Livio, M., Piran, T., \& Schramm, D. N., Nucleosynthesis, neutrino bursts and gamma-rays from coalescing neutron stars, Nature, 340, 126, 1989. (Cited on page 45.) 
Einfeldt, B., On Godunov-type methods for gas dynamics, SIAM J. Numer. Anal., 25, 294, 1988. (Cited on page 24.)

Endrizzi, A., Ciolfi, R., Giacomazzo, B., Kastaun, W., \& Kawamura, T., General relativistic magnetohydrodynamic simulations of binary neutron star mergers with the APR4 equation of state, Classical and Quantum Gravity, 33, 164001, arXiv:1604.03445 [astro-ph.HE], 2016. (Cited on pages 45 and 64.)

Essick, R., \& Landry, P., Discriminating between Neutron Stars and Black Holes with Imperfect Knowledge of the Maximum Neutron Star Mass, arXiv e-prints, arXiv:2007.01372, arXiv:2007.01372 [astro-ph.HE], 2020. (Cited on page 59.)

Etienne, Z. B., Paschalidis, V., Haas, R., Mösta, P., \& Shapiro, S. L., IllinoisGRMHD: an open-source, user-friendly GRMHD code for dynamical spacetimes, Class. Quantum Grav., 32, 175009, arXiv:1501.07276 [astro-ph.HE], 2015. (Cited on page 23.)

Etienne, Z. B., Paschalidis, V., Liu, Y. T., \& Shapiro, S. L., Relativistic magnetohydrodynamics in dynamical spacetimes: Improved electromagnetic gauge condition for adaptive mesh refinement grids, Phys. Rev. D, 85, 024013, arXiv:1110.4633 [astro-ph.HE], 2012. (Cited on page 23.)

Event Horizon Telescope Collaboration, Akiyama, K., Alberdi, A., et al., First M87 Event Horizon Telescope Results. I. The Shadow of the Supermassive Black Hole, Astrophys. J. Lett., 875, L1, 2019. (Cited on page 1.)

Event Horizon Telescope Collaboration, Akiyama, K., Alberdi, A., et al., First M87 Event Horizon Telescope Results. II. Array and Instrumentation, Astrophys. J. Lett., 875, L2, 2019. (Cited on page 1.)

Event Horizon Telescope Collaboration, Akiyama, K., Alberdi, A., et al., First M87 Event Horizon Telescope Results. III. Data Processing and Calibration, Astrophys. J. Lett., 875, L3, 2019. (Cited on page 1.)

Event Horizon Telescope Collaboration, Akiyama, K., Alberdi, A., et al., First M87 Event Horizon Telescope Results. IV. Imaging the Central Supermassive Black Hole, Astrophys. J. Lett., 875, L4, 2019. (Cited on page 1.)

Event Horizon Telescope Collaboration, Akiyama, K., Alberdi, A., et al., First M87 Event Horizon Telescope Results. V. Physical Origin of the Asymmetric Ring, Astrophys. J. Lett., 875, L5, 2019. (Cited on page 1.)

Event Horizon Telescope Collaboration, Akiyama, K., Alberdi, A., et al., First M87 Event Horizon Telescope Results. VI. The Shadow and Mass of the Central Black Hole, Astrophys. J. Lett., 875, L6, 2019. (Cited on page 1.)

Farajian, A. H., Bigdeli, M., \& Belbasi, S., LOCV calculation of the equations of state and properties of rapidly rotating neutron stars, Chinese Physics C, 42, 065102, arXiv:1804.04325 [nucl-th], 2018. (Cited on page 47.)

Farris, B. D., Li, T. K., Liu, Y. T., \& Shapiro, S. L., Relativistic radiation magnetohydrodynamics in dynamical spacetimes: Numerical methods and tests, Phys. Rev. D, 78, 024023, arXiv:0802.3210, 2008. (Cited on page 34.)

Farrow, N., Zhu, X.-J., \& Thrane, E., The Mass Distribution of Galactic Double Neutron Stars, Astrophys. J., 876, 18, arXiv:1902.03300 [astro-ph.HE], 2019. (Cited on page 59.) 
Fasano, M., Abdelsalhin, T., Maselli, A., \& Ferrari, V., Constraining the Neutron Star Equation of State Using Multiband Independent Measurements of Radii and Tidal Deformabilities, Phys. Rev. L, 123, 141101, arXiv:1902.05078 [astro-ph.HE], 2019. (Cited on page 51.)

Fattoyev, F. J., Horowitz, C. J., Piekarewicz, J., \& Reed, B., GW190814: Impact of a 2.6 solar mass neutron star on nucleonic equations of state, arXiv e-prints, arXiv:2007.03799, arXiv:2007.03799 [nucl-th], 2020. (Cited on page 59.)

Fattoyev, F. J., Piekarewicz, J., \& Horowitz, C. J., Neutron Skins and Neutron Stars in the Multimessenger Era, Physical Review Letters, 120, 172702, arXiv:1711.06615 [nucl-th], 2018. (Cited on page 50.)

Favata, M., Systematic Parameter Errors in Inspiraling Neutron Star Binaries, Phys. Rev. Lett., 112, 101101, arXiv:1310.8288 [gr-qc], 2014. (Cited on page 42.)

Fernández, R., Tchekhovskoy, A., Quataert, E., Foucart, F., \& Kasen, D., Long-term GRMHD simulations of neutron star merger accretion discs: implications for electromagnetic counterparts, Mon. Not. R. Astron. Soc., 482, 3373, arXiv:1808.00461 [astro-ph.HE], 2019. (Cited on page 5.)

Figura, A., Lu, J. J., Burgio, G. F., Li, Z. H., \& Schulze, H. J., Hybrid equation of state approach in binary neutron-star merger simulations, arXiv e-prints, arXiv:2005.08691, arXiv:2005.08691 [gr-qc], 2020. (Cited on pages 64 and 128.)

Fischer, T., Bastian, N.-U. F., Wu, M.-R., et al., Quark deconfinement as a supernova explosion engine for massive blue supergiant stars, Nature Astronomy, 2, 980, arXiv:1712.08788 [astro-ph.HE], 2018. (Cited on page 62.)

Flanagan, É. É., \& Hinderer, T., Constraining neutron-star tidal Love numbers with gravitational-wave detectors, Physical Review D, 77, 021502, arXiv:0709.1915 [astro-ph], 2008. (Cited on page 42.)

Fleck, Jr., J. A., \& Cummings, Jr., J. D., An implicit monte carlo scheme for calculating time and frequency dependent nonlinear radiation transport, J. Comput. Phys., 8, 313, 1971. (Cited on page 31.)

Font, J. A., Ibanez, J. M., Marquina, A., \& Marti, J. M., Multidimensional relativistic hydrodynamics: Characteristic fields and modern high-resolution shock-capturing schemes, Astron. Astrophys., 282, 304, 1994. (Cited on page 80.)

Fortin, M., Oertel, M., \& Providência, C., Hyperons in hot dense matter: what do the constraints tell us for equation of state?, Pub. Astron. Soc. Aust., 35, E044, arXiv:1711.09427 [astro-ph.HE], 2018. (Cited on page 2.)

Foucart, F., Monte Carlo closure for moment-based transport schemes in general relativistic radiation hydrodynamic simulations, Mon. Not. R. Astron. Soc., 475, 4186, arXiv:1708.08452 [astro-ph.HE], 2018. (Cited on page 31.)

Foucart, F., Duez, M. D., Hebert, F., et al., Monte-carlo neutrino transport in neutron star merger simulations, arXiv:2008.08089 [astro-ph.HE], 2020. (Cited on page 31.)

Foucart, F., Duez, M. D., Kidder, L. E., et al., Evaluating radiation transport errors in merger simulations using a Monte Carlo algorithm, Phys. Rev. D, 98, 063007, arXiv:1806.02349 [astro-ph.HE], 2018. (Cited on pages 31, 80, 88, and 102.) 
Foucart, F., O'Connor, E., Roberts, L., et al., Impact of an improved neutrino energy estimate on outflows in neutron star merger simulations, Phys. Rev. D, 94, 123016, arXiv:1607.07450 [astro-ph.HE], 2016. (Cited on pages 75 and 86.)

Foucart, F., Deaton, M. B., Duez, M. D., et al., Black-hole-neutron-star mergers at realistic mass ratios: Equation of state and spin orientation effects, Phys. Rev. D, 87, 084006, arXiv:1212.4810 [gr-qc], 2013. (Cited on page 23.)

Foucart, F., O'Connor, E., Roberts, L., et al., Post-merger evolution of a neutron star-black hole binary with neutrino transport, Phys. Rev. D, 91, 124021, arXiv:1502.04146 [astro-ph.HE], 2015. (Cited on pages 30, 75, 79, 81, 82, 84, 85, 86, $88,114,125,175$, and 176.)

Foucart, F., Haas, R., Duez, M. D., et al., Low mass binary neutron star mergers: Gravitational waves and neutrino emission, Phys. Rev. D, 93, 044019, arXiv:1510.06398 [astro-ph.HE], 2016. (Cited on page 75.)

Fraga, E. S., Kurkela, A., \& Vuorinen, A., Interacting Quark Matter Equation of State for Compact Stars, Astrophys. J. Lett., 781, L25, arXiv:1311.5154 [nucl-th], 2014. (Cited on pages 38 and 39.)

Fragile, P. C., Gillespie, A., Monahan, T., Rodriguez, M., \& Anninos, P., Numerical Simulations of Optically Thick Accretion onto a Black Hole. I. Spherical Case, Astrophys. J., Supp., 201, 9, arXiv:1204.5538 [astro-ph.IM], 2012. (Cited on pages 30, $75,80,94,96,97,125$, and 175.$)$

Fragile, P. C., Olejar, A., \& Anninos, P., Numerical Simulations of Optically Thick Accretion onto a Black Hole. II. Rotating Flow, Astrophys. J., 796, 22, arXiv:1408.4460 [astro-ph.IM], 2014. (Cited on pages 30, 75, 88, 114, and 175.)

Friedman, J. L., Ipser, J. R., \& Sorkin, R. D., Turning-point method for axisymmetric stability of rotating relativistic stars, Astrophys. J., 325, 722, 1988. (Cited on pages 42 and 43.)

Friedman, J. L., \& Stergioulas, N., Rotating Relativistic Stars, 2013. (Cited on page 43.)

Fromm, C. M., Perucho, M., Porth, O., et al., Jet-torus connection in radio galaxies. Relativistic hydrodynamics and synthetic emission, Astron. Astrophys., 609, A80, arXiv:1705.01300 [astro-ph.HE], 2018. (Cited on page 123.)

Fujibayashi, S., Sekiguchi, Y., Kiuchi, K., \& Shibata, M., Properties of Neutrinodriven Ejecta from the Remnant of a Binary Neutron Star Merger: Pure Radiation Hydrodynamics Case, Astrophys. J., 846, 114, 2017. (Cited on pages 5, 6, 32, 74, 126, and 175.)

Gabbana, A., Mendoza, M., Succi, S., \& Tripiccione, R., Numerical evidence of electron hydrodynamic whirlpools in graphene samples, Computers \& Fluids, 172, 644 , 2018. (Cited on page 108.)

Gabbana, A., Simeoni, D., Succi, S., \& Tripiccione, R., Relativistic lattice Boltzmann methods: Theory and applications, Physics Reports, 863, 1, arXiv:1909.04502, 2020. (Cited on pages 32 and 102.)

Gaitanos, T., Di Toro, M., Typel, S., et al., On the Lorentz structure of the symmetry energy, Nuclear Physics A, 732, 24, arXiv:nucl-th/0309021, 2004. (Cited on page 47.) 
Galeazzi, F., Kastaun, W., Rezzolla, L., \& Font, J. A., Implementation of a simplified approach to radiative transfer in general relativity, Phys. Rev. D, 88, 064009, arXiv:1306.4953 [gr-qc], 2013. (Cited on page 29.)

Gamba, I. M., Haack, J. R., Hauck, C. D., \& Hu, J., A fast spectral method for the boltzmann collision operator with general collision kernels, SIAM Journal on Scientific Computing, 39, B658, 2017. (Cited on page 110.)

Gao, H., Ai, S.-K., Cao, Z.-J., et al., Relation between gravitational mass and baryonic mass for non-rotating and rapidly rotating neutron stars, Frontiers of Physics, 15, 24603, arXiv:1905.03784 [astro-ph.HE], 2020. (Cited on page 47.)

Gendreau, K. C., Arzoumanian, Z., Adkins, P. W., et al., The Neutron star Interior Composition Explorer (NICER): design and development, in Proceedings of the SPIE, Vol. 9905, Space Telescopes and Instrumentation 2016: Ultraviolet to Gamma Ray 99051H, 2016. (Cited on page 51.)

Giacomazzo, B., \& Rezzolla, L., WhiskyMHD: a new numerical code for general relativistic magnetohydrodynamics, Class. Quantum Grav., 24, 235, arXiv:grqc/0701109, 2007. (Cited on page 23.)

Gill, R., Nathanail, A., \& Rezzolla, L., When Did the Remnant of GW170817 Collapse to a Black Hole?, Astrophys. J., 876, 139, arXiv:1901.04138 [astro-ph.HE], 2019. (Cited on page 5.)

Gillman, A. W., \& Stellingwerf, R. F., Relativistic accretion - The optically thick case, Astrophys. J., 240, 235, 1980. (Cited on page 94.)

Glendenning, N., Compact stars: Nuclear physics, particle physics, and general relativity (Springer New York), 2000. (Cited on page 1.)

Glendenning, N. K., First order phase transitions with more than one conserved charge: Consequences for neutron stars, Phys. Rev., D46, 1274, 1992. (Cited on page 63.)

Godenschwager, C., Schornbaum, F., Bauer, M., Köstler, H., \& Rüde, U., in Proceedings of the International Conference on High Performance Computing, Networking, Storage and Analysis, SC '13A Framework for Hybrid Parallel Flow Simulations with a Trillion Cells in Complex Geometries, (New York, NY, USA: Association for Computing Machinery) 35, 2013. (Cited on page 120.)

Godunov, S. K., A difference method for numerical calculations of discontinuous solutions of the equations of hydrpdynamics, Mat. Sb., 47, 271, 1959. (Cited on page 24.)

Godzieba, D. A., Radice, D., \& Bernuzzi, S., On the maximum mass of neutron stars and GW190814, arXiv e-prints, arXiv:2007.10999, arXiv:2007.10999 [astro-ph.HE], 2020. (Cited on page 59.)

Goldstein, A., Veres, P., Burns, E., et al., An Ordinary Short Gamma-Ray Burst with Extraordinary Implications: Fermi-GBM Detection of GRB 170817A, Astrophys. J. Letters, 848, L14, arXiv:1710.05446 [astro-ph.HE], 2017. (Cited on page 45.)

Gómez, R., Lehner, L., Papadopoulos, P., \& Winicour, J., The eth formalism in numerical relativity, Class. Quantum Grav., 14, 977, arXiv:gr-qc/9702002, 1997. (Cited on page 21.) 
Gondek-Rosińska, D., Kowalska, I., Villain, L., Ansorg, M., \& Kucaba, M., A New View on the Maximum Mass of Differentially Rotating Neutron Stars, Astrophys. J., 837, 58, arXiv:1609.02336 [astro-ph.HE], 2017. (Cited on page 44.)

Gourgoulhon, E., Grandclement, P., Taniguchi, K., Marck, J.-A., \& Bonazzola, S., Quasiequilibrium sequences of synchronized and irrotational binary neutron stars in general relativity. I. Method and tests, Phys. Rev. D, 63, 064029, arXiv:grqc/0007028, 2001. (Cited on page 65.)

Grad, H., Note on n-dimensional hermite polynomials, Communications on Pure and Applied Mathematics, 2, 325, 1949. (Cited on page 103.)

Grad, H., On the kinetic theory of rarefied gases, Communications on Pure and Applied Mathematics, 2, 331, 1949. (Cited on page 103.)

Greif, S. K., Raaijmakers, G., Hebeler, K., Schwenk, A., \& Watts, A. L., Equation of state sensitivities when inferring neutron star and dense matter properties, Mon. Not. R. Astron. Soc., 485, 5363, arXiv:1812.08188 [astro-ph.HE], 2019. (Cited on pages 38,52 , and 54 .)

Gross, B., \& Atzberger, P., Hydrodynamic flows on curved surfaces: Spectral numerical methods for radial manifold shapes, Journal of Computational Physics, 371, 663 , 2018. (Cited on page 110.)

Guillot, S., Servillat, M., Webb, N. A., \& Rutledge, R. E., Measurement of the Radius of Neutron Stars with High Signal-to-noise Quiescent Low-mass X-Ray Binaries in Globular Clusters, Astrophys. J., 772, 7, arXiv:1302.0023 [astro-ph.HE], 2013. (Cited on pages 3 and 51.)

Guillot, S., Kerr, M., Ray, P. S., et al., NICER X-Ray Observations of Seven Nearby Rotation-powered Millisecond Pulsars, Astrophys. J. Lett., 887, L27, arXiv:1912.05708 [astro-ph.HE], 2019. (Cited on pages 51, 57, 58, and 173.)

Gulminelli, F., \& Raduta, A. R., Unified treatment of subsaturation stellar matter at zero and finite temperature, Phys. Rev. C, 92, 055803, arXiv:1504.04493 [nucl-th], 2015. (Cited on page 47.)

Gundlach, C., Martin-Garcia, J. M., Calabrese, G., \& Hinder, I., Constraint damping in the Z4 formulation and harmonic gauge, Class. Quantum Grav., 22, 3767, arXiv:gr-qc/0504114, 2005. (Cited on page 16.)

Hairer, E., Norsett, S. P., \& Wanner, G., Solving ordinary differential equations $i$ nonstiff problems (Springer), 1993. (Cited on page 25.)

Han, M.-Z., Tang, S.-P., Hu, Y.-M., et al., Is GW190425 consistent with being a neutron star-black hole merger?, arXiv e-prints, arXiv:2001.07882, arXiv:2001.07882 [astro-ph.HE], 2020. (Cited on page 129.)

Hanauske, M., Takami, K., Bovard, L., et al., Rotational properties of hypermassive neutron stars from binary mergers, Phys. Rev. D, 96, 043004, arXiv:1611.07152 [gr-qc], 2017. (Cited on pages 4, 45, 46, 47, 64, 67, 70, 172, and 174.)

Hanauske, M., Weih, L. R., Stöcker, H., \& Rezzolla, L., Metastable hypermassive hybrid stars as neutron-star merger remnants, European Physical Journal, in press, 2021. (Cited on pages 60, 68, 69, and 174.) 
Hanauske, M., Steinheimer, J., Bovard, L., et al., Concluding Remarks: Connecting Relativistic Heavy Ion Collisions and Neutron Star Mergers by the Equation of State of Dense Hadron- and Quark Matter as signalled by Gravitational Waves, in Journal of Physics Conference Series, Vol. 878, Journal of Physics Conference Series 012031, 2017. (Cited on page 70.)

Hanauske, M., Bovard, L., Steinheimer, J., et al., MAGIC - how MAtter's extreme phases can be revealed in Gravitational wave observations and in relativistic heavy Ion Collision experiments, in Journal of Physics Conference Series, Vol. 1271, Journal of Physics Conference Series 012023, 2019. (Cited on page 174.)

Harris, S., An introduction to the theory of the Boltzmann equation. (New York: Holt, Rinehart and Winston, Inc.), 1971. (Cited on page 26.)

Harten, A., Lax, P. D., \& van Leer, B., On upstream differencing and godunov-type schemes for hyperbolic conservation laws, SIAM Rev., 25, 35, 1983. (Cited on page 24.)

He, X., Chen, S., \& Doolen, G. D., A novel thermal model for the lattice boltzmann method in incompressible limit, Journal of Computational Physics, 146, 282 , 1998. (Cited on page 104.)

He, X., \& Luo, L.-S., Theory of the lattice Boltzmann method: From the Boltzmann equation to the lattice Boltzmann equation, Phys. Rev. E, 56, 6811, 1997. (Cited on page 103.)

Hebeler, K., Lattimer, J. M., Pethick, C. J., \& Schwenk, A., Constraints on Neutron Star Radii Based on Chiral Effective Field Theory Interactions, Phys. Rev. Lett., 105, 161102, arXiv:1007.1746 [nucl-th], 2010. (Cited on page 38.)

Hempel, M., \& Schaffner-Bielich, J., A statistical model for a complete supernova equation of state, Nuclear Physics A, 837, 210, arXiv:0911.4073 [nucl-th], 2010. (Cited on page 47.)

Hessels, J. W., Ransom, S. M., Stairs, I. H., et al., A Radio Pulsar Spinning at 716 $H z$, Science, 311, 1901, 2006. (Cited on page 2.)

Hildebrand, F. B., Introduction to numerical analysis (New York : McGraw-Hill), includes bibliography, 1956. (Cited on page 110.)

Hilditch, D., An Introduction to Well-Posedness and Free-Evolution, International Journal of Modern Physics A, 28, 1340015, arXiv:1309.2012 [gr-qc], 2013. (Cited on page 15.)

Hilditch, D., Bernuzzi, S., Thierfelder, M., et al., Compact binary evolutions with the Z4c formulation, Phys. Rev. D, 88, 084057, arXiv:1212.2901 [gr-qc], 2013. (Cited on page 17.)

Hilditch, D., \& Ruiz, M., The initial boundary value problem for free-evolution formulations of general relativity, Classical and Quantum Gravity, 35, 015006, arXiv:1609.06925 [gr-qc], 2018. (Cited on page 18.)

Hinderer, T., Tidal Love Numbers of Neutron Stars, Astrophys. J., 677, 1216, arXiv:0711.2420, 2008. (Cited on page 41.) 
Hinderer, T., Lackey, B. D., Lang, R. N., \& Read, J. S., Tidal deformability of neutron stars with realistic equations of state and their gravitational wave signatures in binary inspiral, Phys. Rev. D, 81, 123016, arXiv:0911.3535 [astro-ph.HE], 2010. (Cited on page 41.)

Hou, T. Y., \& LeFloch, P. G., Why Nonconservative Schemes Converge to Wrong Solutions: Error Analysis, Math. Comp., 62, 497, 1994. (Cited on page 22.)

Iwakami, W., Okawa, H., Nagakura, H., et al., Simulations of the Early PostBounce Phase of Core-Collapse Supernovae in Three-Dimensional Space with Full Boltzmann Neutrino Transport, arXiv e-prints, arXiv:2004.02091, arXiv:2004.02091 [astro-ph.HE], 2020. (Cited on pages 32 and 110.)

Janka, H. T., Flux-limited neutrino diffusion versus Monte Carlo neutrino transport, Astron. Astrophys., 256, 452, 1992. (Cited on page 31.)

Janka, H. T., \& Hillebrandt, W., Monte Carlo simulations of neutrino transport in type II supernovae., Astronomy and Astrophysics, Supplement, 78, 375, 1989. (Cited on page 31.)

Janka, H.-T., Langanke, K., Marek, A., Martínez-Pinedo, G., \& Müller, B., Theory of core-collapse supernovae, Physics Reports, 442, 38, arXiv:arXiv:astro-ph/0612072, 2007. (Cited on page 2.)

Jin, S., \& Levermore, C. D., Numerical Schemes for Hyperbolic Conservation Laws with Stiff Relaxation Terms., Journal of Computational Physics, 126, 449, 1996. (Cited on page 81.)

Johnston, S., Lorimer, D. R., Harrison, P. A., et al., Discovery of a very bright, nearby binary millisecond pulsar, Nature, 361, 613, 1993. (Cited on page 53.)

Just, O., Bauswein, A., Pulpillo, R. A., Goriely, S., \& Janka, H.-T., Comprehensive nucleosynthesis analysis for ejecta of compact binary mergers, Mon. Not. R. Astron. Soc., 448, 541, arXiv:1406.2687 [astro-ph.SR], 2015. (Cited on pages 5 and 172.)

Just, O., Obergaulinger, M., \& Janka, H. T., A new multidimensional, energydependent two-moment transport code for neutrino-hydrodynamics, Mon. Not. R. Astron. Soc., 453, 3386, arXiv:1501.02999 [astro-ph.HE], 2015. (Cited on page 75.)

Karlin, I. V., Sichau, D., \& Chikatamarla, S. S., Consistent two-population lattice boltzmann model for thermal flows, Phys. Rev. E, 88, 063310, 2013. (Cited on page 104.)

Kasen, D., Metzger, B., Barnes, J., Quataert, E., \& Ramirez-Ruiz, E., Origin of the heavy elements in binary neutron-star mergers from a gravitational-wave event, Nature, 551, 80, arXiv:1710.05463 [astro-ph.HE], 2017. (Cited on page 45.)

Kastaun, W., Ciolfi, R., Endrizzi, A., \& Giacomazzo, B., Structure of stable binary neutron star merger remnants: Role of initial spin, Phys. Rev. D, 96, 043019, arXiv:1612.03671 [astro-ph.HE], 2017. (Cited on pages 67, 70, and 174.)

Kastaun, W., Ciolfi, R., \& Giacomazzo, B., Structure of stable binary neutron star merger remnants: A case study, Phys. Rev. D, 94, 044060, arXiv:1607.02186 [astroph.HE], 2016. (Cited on page 70.) 
Kastaun, W., \& Galeazzi, F., Properties of hypermassive neutron stars formed in mergers of spinning binaries, Phys. Rev. D, 91, 064027, arXiv:1411.7975 [gr-qc], 2015. (Cited on page 4.)

Kawaguchi, K., Kiuchi, K., Kyutoku, K., et al., Frequency-domain gravitational waveform models for inspiraling binary neutron stars, Phys. Rev. D, 97, 044044, arXiv:1802.06518 [gr-qc], 2018. (Cited on pages 5 and 70.)

Keppens, R., Meliani, Z., van Marle, A. J., et al., Parallel, grid-adaptive approaches for relativistic hydro and magnetohydrodynamics, Journal of Computational Physics, 231, 718, 2012. (Cited on page 86.)

Kiuchi, K., Kyutoku, K., Sekiguchi, Y., Shibata, M., \& Wada, T., High resolution numerical relativity simulations for the merger of binary magnetized neutron stars, Phys. Rev. D, 90, 041502, arXiv:1407.2660 [astro-ph.HE], 2014. (Cited on page 64.)

Kiuchi, K., Kyutoku, K., Shibata, M., \& Taniguchi, K., Revisiting the Lower Bound on Tidal Deformability Derived by AT 2017gfo, Astrophys. J., 876, L31, arXiv:1903.01466 [astro-ph.HE], 2019. (Cited on page 49.)

Kiuchi, K., Sekiguchi, Y., Kyutoku, K., \& Shibata, M., Gravitational waves, neutrino emissions and effects of hyperons in binary neutron star mergers, Class. Quantum Grav., 29, 124003, arXiv:1206.0509 [astro-ph.HE], 2012. (Cited on page 2.)

Knapp, A. M., Walker, E. J., \& Baumgarte, T. W., Illustrating stability properties of numerical relativity in electrodynamics, Phys. Rev. D, 65, 064031, 2002. (Cited on page 15.)

Koeppel, S., Bovard, L., \& Rezzolla, L., A General-relativistic Determination of the Threshold Mass to Prompt Collapse in Binary Neutron Star Mergers, Astrophys. J. Lett., 872, L16, arXiv:1901.09977 [gr-qc], 2019. (Cited on pages 3, 45, 50, and 51.)

Kohno, M., Nuclear and neutron matter G-matrix calculations with a chiral effective field theory potential including effects of three-nucleon interactions, Physical Review C, 88, 064005, arXiv:1309.4556 [nucl-th], 2013. (Cited on page 39.)

Kolgan, V. P., Application of the minimum-derivative principle in the construction of finite-difference schemes for numerical analysis of discontinuous solutions in gas dynamics, Transactions of the Central Aerohydrodynamics Institute, 3, 68, 1972. (Cited on page 24.)

Koliogiannis, P. S., \& Moustakidis, C. C., Effects of the equation of state on the bulk properties of maximally rotating neutron stars, Phys. Rev. C, 101, 015805, arXiv:1907.13375 [nucl-th], 2020. (Cited on pages 44 and 47.)

Komatsu, H., Eriguchi, Y., \& Hachisu, I., Rapidly rotating general relativistic stars. I - Numerical method and its application to uniformly rotating polytropes, Mon. Not. R. Astron. Soc., 237, 355, 1989. (Cited on page 43.)

Krüger, T., Kusumaatmaja, H., Kuzmin, A., et al., The lattice boltzmann method (Springer International Publishing), 2017. (Cited on pages 32, 102, 104, and 176.)

Kurkela, A., Fraga, E. S., Schaffner-Bielich, J., \& Vuorinen, A., Constraining Neutron Star Matter with Quantum Chromodynamics, Astrophys. J., 789, 127, 2014. (Cited on page 39.) 
Kurkela, A., Romatschke, P., \& Vuorinen, A., Cold quark matter, Phys. Rev. D, 81, 105021, arXiv:0912.1856 [hep-ph], 2010. (Cited on pages 38 and 39.)

Kuroda, T., Takiwaki, T., \& Kotake, K., A New Multi-energy Neutrino RadiationHydrodynamics Code in Full General Relativity and Its Application to the Gravitational Collapse of Massive Stars, Astrophys. J., Supp., 222, 20, arXiv:1501.06330 [astro-ph.HE], 2016. (Cited on page 75.)

Kyutoku, K., Fujibayashi, S., Hayashi, K., et al., On the possibility of GW190425 being a black hole-neutron star binary merger, arXiv e-prints, arXiv:2001.04474, arXiv:2001.04474 [astro-ph.HE], 2020. (Cited on page 129.)

Lasota, J.-P., Haensel, P., \& Abramowicz, M. A., Fast Rotation of Neutron Stars, Astrophys. J., 456, 300, arXiv:astro-ph/9508118, 1996. (Cited on page 47.)

Lattimer, J. M., Introduction to neutron stars, in American Institute of Physics Conference Series, Vol. 1645, American Institute of Physics Conference Series 61, 2015. (Cited on page 38.)

Lattimer, J. M., \& Prakash, M., Neutron Star Structure and the Equation of State, Astrophys. J., 550, 426, arXiv:astro-ph/0002232, 2001. (Cited on page 2.)

Lattimer, J. M., \& Prakash, M., The Physics of Neutron Stars, Science, 304, 536, arXiv:arXiv:astro-ph/0405262, 2004. (Cited on page 55.)

Lattimer, J. M., \& Prakash, M., The equation of state of hot, dense matter and neutron stars, Physics Reports, 621, 127, arXiv:1512.07820 [astro-ph.SR], 2016. (Cited on page 35.)

Lattimer, J. M., \& Steiner, A. W., Neutron Star Masses and Radii from Quiescent Low-mass X-Ray Binaries, Astrophys. J., 784, 123, arXiv:1305.3242 [astro-ph.HE], 2014. (Cited on pages 3 and 51.)

Lax, P. D., \& Wendroff, B., Systems of conservation laws, Commun. Pure Appl. Math., 13, 217, 1960. (Cited on page 22.)

Lebedev, V., Values of the nodes and weights of ninth to seventeenth order gaussmarkov quadrature formulae invariant under the octahedron group with inversion, USSR Computational Mathematics and Mathematical Physics, 15, 44, 1975. (Cited on page 110.)

Lebedev, V., Quadratures on a sphere, USSR Computational Mathematics and Mathematical Physics, 16, 10 , 1976. (Cited on page 110.)

Lebedev, V. I., Spherical quadrature formulas exact to orders 25-29, Siberian Mathematical Journal, 18, 99, 1977. (Cited on page 110.)

Lenka, S. S., Char, P., \& Banik, S., Critical mass, moment of inertia and universal relations of rapidly rotating neutron stars with exotic matter, International Journal of Modern Physics D, 26, 1750127, arXiv:1704.07113 [astro-ph.HE], 2017. (Cited on page 47.)

Levermore, C. D., Relating Eddington factors to flux limiters., Journal of Quantitative Spectroscopy and Radiative Transfer, 31, 149, 1984. (Cited on pages 30, 79, and 175.)

Levermore, C. D., \& Pomraning, G. C., A flux-limited diffusion theory, Astrophys. J., 248, 321, 1981. (Cited on page 30.) 
Lewin, W. H. G., van Paradijs, J., \& Taam, R. E., X-Ray Bursts, Space Sci. Rev., 62, 223, 1993. (Cited on page 3.)

Liebendörfer, M., Whitehouse, S. C., \& Fischer, T., The Isotropic Diffusion Source Approximation for Supernova Neutrino Transport, Astrophys. J., 698, 1174, arXiv:0711.2929, 2009. (Cited on page 30.)

LIGO Scientific Collaboration, Virgo Collaboration, Gamma-Ray Burst Monitor, et al., Gravitational waves and gamma-rays from a binary neutron star merger: Gw170817 and grb 170817a, Astrophys. J. Lett., 848, L13, arXiv:1710.05834 [astroph.HE], 2017. (Cited on pages 5, 45, 128, and 172.)

Lim, Y., \& Holt, J. W., Neutron Star Tidal Deformabilities Constrained by Nuclear Theory and Experiment, Phys. Rev. Lett., 121, 062701, arXiv:1803.02803 [nucl-th], 2018. (Cited on page 50.)

Lim, Y., \& Holt, J. W., Bayesian modeling of the nuclear equation of state for neutron star tidal deformabilities and GW170817, arXiv e-prints, arXiv:1902.05502, arXiv:1902.05502 [nucl-th], 2019. (Cited on page 50.)

Lindblom, L., Determining the nuclear equation of state from neutron-star masses and radii, Astrophys. J., 398, 569, 1992. (Cited on page 35.)

Lindblom, L., Spectral representations of neutron-star equations of state, Phys. Rev. D, 82, 103011, arXiv:1009.0738 [astro-ph], 2010. (Cited on page 38.)

Lindblom, L., The relativistic inverse stellar structure problem, in American Institute of Physics Conference Series, Vol. 1577, American Institute of Physics Conference Series 153, arXiv:1402.0035 [astro-ph.HE], 2014. (Cited on page 35.)

Lindblom, L., Causal representations of neutron-star equations of state, Phys. Rev. D, 97, 123019, arXiv:1804.04072 [astro-ph.HE], 2018. (Cited on page 38.)

Lindblom, L., \& Indik, N. M., Spectral approach to the relativistic inverse stellar structure problem, Phys. Rev. D, 86, 084003, arXiv:1207.3744 [astro-ph.HE], 2012. (Cited on page 35.)

Lindquist, R. W., Relativistic transport theory, Annals of Physics, 37, 487, 1966. (Cited on page 28.)

Loeffler, F., Faber, J., Bentivegna, E., et al., The Einstein Toolkit: a community computational infrastructure for relativistic astrophysics, Class. Quantum Grav., 29, 115001, arXiv:1111.3344 [gr-qc], 2012. (Cited on pages 64 and 125.)

Lommen, A. N., Zepka, A., Backer, D. C., et al., New Pulsars from an Arecibo Drift Scan Search, Astrophys. J., 545, 1007, arXiv:astro-ph/0008054 [astro-ph], 2000. (Cited on page 53.)

Lorimer, D. R., Binary and millisecond pulsars, Living Rev. Relativ., 8, 2008. (Cited on page 44.)

Lorimer, D. R., \& Kramer, M., Handbook of Pulsar Astronomy, 2012. (Cited on page 2.)

Lutsko, J. F., \& Lam, J., Classical density functional theory, unconstrained crystallization, and polymorphic behavior, Phys. Rev. E, 98, 012604, 2018. (Cited on page 110.) 
Maione, F., De Pietri, R., Feo, A., \& Löffler, F., Spectral analysis of gravitational waves from binary neutron star merger remnants, Phys. Rev. D, 96, 063011, arXiv:1707.03368 [gr-qc], 2017. (Cited on pages 5 and 70.)

Malik, T., Alam, N., Fortin, M., et al., GW170817: Constraining the nuclear matter equation of state from the neutron star tidal deformability, Physical Review C, 98, 035804, arXiv:1805.11963 [nucl-th], 2018. (Cited on page 50.)

Margalit, B., \& Metzger, B. D., Constraining the Maximum Mass of Neutron Stars from Multi-messenger Observations of GW170817, Astrophys. J. Lett., 850, L19, arXiv:1710.05938 [astro-ph.HE], 2017. (Cited on pages 48, 52, 127, and 173.)

Margaritis, C., Koliogiannis, P. S., \& Moustakidis, C. C., Speed of sound constraints on maximally rotating neutron stars, Phys. Rev. D, 101, 043023, arXiv:1910.05767 [nucl-th], 2020. (Cited on page 44.)

Martí, J. M., Ibáñez, J. M., \& Miralles, J. A., Numerical relativistic hydrodynamics: Local characteristic approach, Phys. Rev. D, 43, 3794, 1991. (Cited on page 22.)

Martí, J. M., Müller, E., Font, J. A., Ibáñez, J. M., \& Marquina, A., Morphology and dynamics of relativistic jets, Astrophys. J., 479, 151, 1997. (Cited on pages 122 and 124.)

Massaioli, F., Benzi, R., \& Succi, S., Exponential tails in two-dimensional rayleighbénard convection, Europhysics Letters (EPL), 21, 305, 1993. (Cited on page 104.)

Mazzeo, M., \& Coveney, P., Hemelb: A high performance parallel lattice-boltzmann code for large scale fluid flow in complex geometries, Computer Physics Communications, 178, 894 , 2008. (Cited on page 120.)

McCulloch, R., \& Bindra, H., Coupled radiative and conjugate heat transfer in participating media using lattice boltzmann methods, Computers \& Fluids, 124, 261, 2016. (Cited on page 105.)

McHardy, C., Horneber, T., \& Rauh, C., New lattice Boltzmann method for the simulation of three-dimensional radiation transfer in turbid media, Optics Express, 24, 16999, 2016. (Cited on page 105.)

McKinney, J. C., Tchekhovskoy, A., \& Blandford, R. D., Alignment of Magnetized Accretion Disks and Relativistic Jets with Spinning Black Holes, Science, 339, 49, arXiv:1211.3651 [astro-ph.CO], 2013. (Cited on pages 94, 97, and 98.)

McKinney, J. C., Tchekhovskoy, A., Sadowski, A., \& Narayan, R., Three-dimensional general relativistic radiation magnetohydrodynamical simulation of super-Eddington accretion, using a new code HARMRAD with M1 closure, Mon. Not. R. Astron. Soc., 441, 3177, arXiv:1312.6127, 2014. (Cited on pages 75, 85, 88, 114, 125, and 175.)

McNeil Forbes, M., Bose, S., Reddy, S., et al., Constraining the neutron-matter equation of state with gravitational waves, arXiv e-prints, arXiv:1904.04233, arXiv:1904.04233 [astro-ph.HE], 2019. (Cited on page 51.)

Melon Fuksman, J. D., \& Mignone, A., A Radiative Transfer Module for Relativistic Magnetohydrodynamics in the PLUTO Code, Astrophys. J., Supp., 242, 20, arXiv:1903.10456 [astro-ph.IM], 2019. (Cited on pages 85 and 93.)

Mendoza, M., Karlin, I., Succi, S., \& Herrmann, H. J., Relativistic lattice boltzmann model with improved dissipation, Phys. Rev. D, 87, 065027, 2013. (Cited on page 108.) 
Metzger, B. D., The Kilonova Handbook, ArXiv e-prints, arXiv:1610.09381 [astroph.HE], 2016. (Cited on page 45.)

Metzger, B. D., Kilonovae, Living Reviews in Relativity, 20, 3, arXiv:1610.09381 [astro-ph.HE], 2017. (Cited on pages 45 and 47.)

Metzger, B. D., Welcome to the Multi-Messenger Era! Lessons from a Neutron Star Merger and the Landscape Ahead, ArXiv e-prints, arXiv:1710.05931 [astro-ph.HE], 2017. (Cited on pages 5, 45, and 172.)

Metzger, B. D., \& Fernández, R., Red or blue? A potential kilonova imprint of the delay until black hole formation following a neutron star merger, Mon. Not. R. Astron. Soc., 441, 3444, arXiv:1402.4803 [astro-ph.HE], 2014. (Cited on pages 5 and 6.)

Metzger, B. D., Quataert, E., \& Thompson, T. A., Short-duration gamma-ray bursts with extended emission from protomagnetar spin-down, Mon. Not. R. Astron. Soc., 385, 1455, arXiv:0712.1233, 2008. (Cited on page 45.)

Metzger, B. D., Thompson, T. A., \& Quataert, E., A Magnetar Origin for the Kilonova Ejecta in GW170817, Astrophys. J., 856, 101, arXiv:1801.04286 [astro-ph.HE], 2018. (Cited on page 45.)

Mezzacappa, A., \& Bruenn, S. W., A Numerical Method for Solving the Neutrino Boltzmann Equation Coupled to Spherically Symmetric Stellar Core Collapse, Astrophys. J., 405, 669, 1993. (Cited on page 32.)

Mezzacappa, A., \& Matzner, R. A., Computer Simulation of Time-dependent, Spherically Symmetric Spacetimes Containing Radiating Fluids: Formalism and Code Tests, Astrophys. J., 343, 853, 1989. (Cited on page 28.)

Mezzacappa, A., \& Messer, O. E. B., Neutrino transport in core collapse supernovae., Journal of Computational and Applied Mathematics, 109, 281, 1999. (Cited on page 32.)

Michel, F. C., Accretion of Matter by Condensed Objects, Astrophysics and Space Science, 15, 153, 1972. (Cited on pages 94 and 176.)

Mihalas, D., \& Auer, L. H., On laboratory-frame radiation hydrodynamics, Journal of Quantitative Spectroscopy and Radiative Transfer, 71, 61, 2001. (Cited on pages 122 and 132.)

Mihalas, D., \& Mihalas, B., Foundations of radiation hydrodynamics, 1984. (Cited on pages 33 and 34.)

Miller, J. M., Ryan, B. R., \& Dolence, J. C., $\nu$ bhlight: Radiation GRMHD for Neutrino-driven Accretion Flows, Astrophys. J., Supp., 241, 30, arXiv:1903.09273 [astro-ph.IM], 2019. (Cited on pages 6, 32, and 74.)

Miller, J. M., Ryan, B. R., Dolence, J. C., et al., Full transport model of GW170817like disk produces a blue kilonova, Phys. Rev. D, 100, 023008, arXiv:1905.07477 [astro-ph.HE], 2019. (Cited on page 31.)

Miller, M. C., Chirenti, C., \& Lamb, F. K., Constraining the Equation of State of High-density Cold Matter Using Nuclear and Astronomical Measurements, Astrophys. J., 888, 12, arXiv:1904.08907 [astro-ph.HE], 2020. (Cited on page 38.) 
Miller, M. C., Lamb, F. K., Dittmann, A. J., et al., PSR J0030+0451 Mass and Radius from NICER Data and Implications for the Properties of Neutron Star Matter, Astrophys. J. Lett., 887, L24, arXiv:1912.05705 [astro-ph.HE], 2019. (Cited on pages $51,57,58,173$, and 174 .)

Minerbo, G. N., Maximum entropy Eddington factors., Journal of Quantitative Spectroscopy and Radiative Transfer, 20,541, 1978. (Cited on pages 30, 79, and 175.)

Mink, A., McHardy, C., Bressel, L., Rauh, C., \& Krause, M. J., Radiative transfer lattice boltzmann methods: $3 d$ models and their performance in different regimes of radiative transfer, Journal of Quantitative Spectroscopy and Radiative Transfer, 243, 106810, 2020. (Cited on page 105.)

Mishra, S. C., Poonia, H., Vernekar, R. R., \& Das, A. K., Lattice boltzmann method applied to radiative transport analysis in a planar participating medium, Heat Transfer Engineering, 35, 1267, 2014. (Cited on page 105.)

Misner, C. W., Thorne, K. S., \& Wheeler, J. A., Gravitation (San Francisco: W. H. Freeman), 1973. (Cited on page 10.)

Mizuno, Y., Gómez, J. L., Nishikawa, K.-I., et al., Recollimation Shocks in Magnetized Relativistic Jets, Astrophys. J., 809, 38, arXiv:1505.00933 [astro-ph.HE], 2015. (Cited on page 124.)

Montaña, G., Tolós, L., Hanauske, M., \& Rezzolla, L., Constraining twin stars with GW170817, Phys. Rev. D, 99, 103009, arXiv:1811.10929 [astro-ph.HE], 2019. (Cited on pages $2,50,58$, and 61 .)

Most, E. R., Probing dense matter with binary neutron star mergers, PhD thesis, Frankfurt, 2020. (Cited on pages 23 and 128.)

Most, E. R., Jens Papenfort, L., Dexheimer, V., et al., On the deconfinement phase transition in neutron-star mergers, European Physical Journal A, 56, 59, arXiv:1910.13893 [astro-ph.HE], 2020. (Cited on page 66.)

Most, E. R., Papenfort, L. J., Dexheimer, V., et al., Signatures of Quark-Hadron Phase Transitions in General-Relativistic Neutron-Star Mergers, Physical Review Letters, 122, 061101, arXiv:1807.03684 [astro-ph.HE], 2019. (Cited on pages 60, 62, 73, 127,128 , and 174.)

Most, E. R., Papenfort, L. J., \& Rezzolla, L., Beyond second-order convergence in simulations of magnetized binary neutron stars with realistic microphysics, Mon. Not. R. Astron. Soc., 490, 3588, arXiv:1907.10328 [astro-ph.HE], 2019. (Cited on pages 23 and 125.)

Most, E. R., Papenfort, L. J., Tsokaros, A., \& Rezzolla, L., Impact of High Spins on the Ejection of Mass in GW170817, Astrophys. J., 884, 40, arXiv:1904.04220 [astro-ph.HE], 2019. (Cited on page 6.)

Most, E. R., Papenfort, L. J., Weih, L. R., \& Rezzolla, L., A lower bound on the maximum mass if the secondary in GW190814 was once a rapidly spinning neutron star, arXiv e-prints, arXiv:2006.14601, arXiv:2006.14601 [astro-ph.HE], 2020. (Cited on page 59.)

Most, E. R., Weih, L. R., \& Rezzolla, L., The heavier the better: how to constrain mass ratios and spins of high-mass neutron star mergers, Mon. Not. R. Astron. Soc., 496, L16, arXiv:2003.10391 [astro-ph.HE], 2020. (Cited on pages 59 and 129.) 
Most, E. R., Weih, L. R., Rezzolla, L., \& Schaffner-Bielich, J., New Constraints on Radii and Tidal Deformabilities of Neutron Stars from GW170817, Phys. Rev. Lett., 120, 261103, arXiv:1803.00549 [gr-qc], 2018. (Cited on pages 2, 35, 38, 40, 49, 50, $51,53,54,56,58$, and 173.)

Murchikova, E. M., Abdikamalov, E., \& Urbatsch, T., Analytic closures for M1 neutrino transport, Mon. Not. R. Astron. Soc., 469, 1725, arXiv:1701.07027 [astroph.HE], 2017. (Cited on pages 79 and 91.)

Murguia-Berthier, A., Montes, G., Ramirez-Ruiz, E., De Colle, F., \& Lee, W. H., Necessary Conditions for Short Gamma-Ray Burst Production in Binary Neutron Star Mergers, Astrophys. J., 788, L8, arXiv:1404.0383 [astro-ph.HE], 2014. (Cited on page 45.)

Murguia-Berthier, A., Ramirez-Ruiz, E., De Colle, F., et al., The Fate of the Merger Remnant in GW170817 and its Imprint on the Jet Structure, arXiv e-prints, arXiv:2007.12245, arXiv:2007.12245 [astro-ph.HE], 2020. (Cited on page 5.)

Nagakura, H., Iwakami, W., Furusawa, S., et al., Simulations of Core-collapse Supernovae in Spatial Axisymmetry with Full Boltzmann Neutrino Transport, Astrophys. J., 854, 136, arXiv:1702.01752 [astro-ph.HE], 2018. (Cited on page 32.)

Nakamura, T., Oohara, K., \& Kojima, Y., General Relativistic Collapse to Black Holes and Gravitational Waves from Black Holes, Progress of Theoretical Physics Supplement, 90, 1, 1987. (Cited on page 15.)

Narayan, R., Paczynski, B., \& Piran, T., Gamma-ray bursts as the death throes of massive binary stars, Astrophys. J. Lett., 395, L83, arXiv:astro-ph/9204001, 1992. (Cited on page 45.)

Nättilä, J., Miller, M. C., Steiner, A. W., et al., Neutron star mass and radius measurements from atmospheric model fits to X-ray burst cooling tail spectra, Astron. Astrophys., 608, A31, arXiv:1709.09120 [astro-ph.HE], 2017. (Cited on page 3.)

Negele, J. W., \& Vautherin, D., Neutron star matter at sub-nuclear densities, Nuclear Physics A, 207, 298, 1973. (Cited on page 38.)

Newman, E., \& Penrose, R., Errata: An Approach to Gravitational Radiation by a Method of Spin Coefficients, Journal of Mathematical Physics, 4, 998, 1963. (Cited on page 20.)

Newman, E. T., \& Penrose, R., An approach to gravitational radiation by a method of spin coefficients, J. Math. Phys., 3, 566, 1962. (Cited on page 20.)

Nobili, L., Turolla, R., \& Zampieri, L., Spherical accretion onto black holes - A complete analysis of stationary solutions, Astrophys. J., 383, 250, 1991. (Cited on page 94.)

Obergaulinger, M., Just, O., Janka, H. T., Aloy, M. A., \& Aloy, C., A New Twomoment Scheme with Algebraic Closure for Energy-dependent Multi-flavor Neutrino Transport in Supernovae, in Astronomical Society of the Pacific Conference Series, Vol. 488, 8th International Conference of Numerical Modeling of Space Plasma Flows (ASTRONUM 2013), ed. N. V. Pogorelov, E. Audit, \& G. P. Zank 255, 2014. (Cited on page 102.)

O'Connor, E., An Open-source Neutrino Radiation Hydrodynamics Code for Corecollapse Supernovae, Astrophys. J., Supp., 219, 24, arXiv:1411.7058 [astro-ph.HE], 2015. (Cited on pages 28, 30, 75, 76, 81, 92, 102, 118, and 175.) 
Oechslin, R., Uryū, K., Poghosyan, G. S., \& Thielemann, F. K., The Influence of quark matter at high densities on binary neutron star mergers, Mon. Not. Roy. Astron. Soc., 349, 1469, arXiv:astro-ph/0401083 [astro-ph], 2004. (Cited on page 62.)

Oertel, M., Hempel, M., Klähn, T., \& Typel, S., Equations of state for supernovae and compact stars, Reviews of Modern Physics, 89, 015007, arXiv:1610.03361 [astroph.HE], 2017. (Cited on pages 2 and 37.)

Oppenheimer, J. R., \& Volkoff, G. M., On Massive Neutron Cores, Physical Review, 55, 374, 1939. (Cited on page 36.)

Özel, F., Baym, G., \& Güver, T., Astrophysical measurement of the equation of state of neutron star matter, Phys. Rev. D, 82, 101301, arXiv:1002.3153 [astro-ph.HE], 2010. (Cited on page 35.)

Özel, F., \& Freire, P., Masses, Radii, and the Equation of State of Neutron Stars, Annual Review of Astronomy and Astrophysics, 54, 401, arXiv:1603.02698 [astroph.HE], 2016. (Cited on pages 35 and 53.)

Özel, F., \& Psaltis, D., Reconstructing the neutron-star equation of state from astrophysical measurements, Phys. Rev. D, 80, 103003, arXiv:0905.1959 [astro-ph.HE], 2009. (Cited on pages 35 and 51.)

Özel, F., Psaltis, D., Güver, T., et al., The Dense Matter Equation of State from Neutron Star Radius and Mass Measurements, Astrophys. J., 820, 28, arXiv:1505.05155 [astro-ph.HE], 2016. (Cited on pages 3 and 51.)

Paardekooper, J. P., Kruip, C. J. H., \& Icke, V., SimpleX2: radiative transfer on an unstructured, dynamic grid, Astron. Astrophys., 515, A79, arXiv:0912.4273 [astroph.CO], 2010. (Cited on page 29.)

Palenzuela, C., Lehner, L., Reula, O., \& Rezzolla, L., Beyond ideal MHD: towards a more realistic modelling of relativistic astrophysical plasmas, Mon. Not. R. Astron. Soc., 394, 1727, arXiv:0810.1838, 2009. (Cited on pages 25, 82, and 85.)

Palenzuela, C., Liebling, S. L., Neilsen, D., et al., Effects of the microphysical equation of state in the mergers of magnetized neutron stars with neutrino cooling, Phys. Rev. D, 92, 044045, arXiv:1505.01607 [gr-qc], 2015. (Cited on page 45.)

Papazoglou, P., Zschiesche, D., Schramm, S., et al., Nuclei in a chiral SU(3) model, Phys. Rev., C59, 411, arXiv:nucl-th/9806087 [nucl-th], 1999. (Cited on page 62.)

Pareschi, L., \& Russo, G., Implicit-explicit runge-kutta schemes and applications to hyperbolic systems with relaxation, Journal of Scientific Computing, 25, 129, 2005. (Cited on pages 25, 82, 113, 125, and 176.)

Paschalidis, V., General relativistic simulations of compact binary mergers as engines for short gamma-ray bursts, Classical and Quantum Gravity, 34, 084002, arXiv:1611.01519 [astro-ph.HE], 2017. (Cited on page 62.)

Paschalidis, V., Ruiz, M., \& Shapiro, S. L., Relativistic Simulations of Black HoleNeutron Star Coalescence: The Jet Emerges, Astrophys. J. Lett., 806, L14, arXiv:1410.7392 [astro-ph.HE], 2015. (Cited on page 128.)

Peng, Y., Shu, C., \& Chew, Y. T., Simplified thermal lattice boltzmann model for incompressible thermal flows, Phys. Rev. E, 68, 026701, 2003. (Cited on page 104.) 
Perego, A., Bernuzzi, S., \& Radice, D., Thermodynamics conditions of matter in neutron star mergers, Eur. Phys. J., A55, 124, arXiv:1903.07898 [gr-qc], 2019. (Cited on pages 4 and 61 .)

Perego, A., Cabezón, R. M., \& Käppeli, R., An Advanced Leakage Scheme for Neutrino Treatment in Astrophysical Simulations, The Astrophysical Journal Supplement Series, 223, 22, arXiv:1511.08519 [astro-ph.IM], 2016. (Cited on page 29.)

Perego, A., Rosswog, S., Cabezón, R. M., et al., Neutrino-driven winds from neutron star merger remnants, Mon. Not. R. Astron. Soc., 443, 3134, arXiv:1405.6730 [astroph.HE], 2014. (Cited on pages 6, 32, and 74.)

Perucho, M., Dissipative Processes and Their Role in the Evolution of Radio Galaxies, Galaxies, 7, 70, arXiv:1907.13599 [astro-ph.HE], 2019. (Cited on page 122.)

Peters, P. C., Gravitational Radiation and the Motion of Two Point Masses, Physical Review, 136, 1224, 1964. (Cited on page 3.)

Philippi, P. C., Hegele, L. A., dos Santos, L. O. E., \& Surmas, R., From the continuous to the lattice boltzmann equation: The discretization problem and thermal models, Phys. Rev. E, 73, 056702, 2006. (Cited on page 107.)

Pomraning, G. C., Maximum entropy Eddington factors and flux limited diffusion theory., Journal of Quantitiative Spectroscopy and Radiative Transfer, 26, 385, 1981. (Cited on page 30.)

Pons, J. A., Ibáñez, J. M., \& Miralles, J. A., Hyperbolic character of the angular moment equations of radiative transfer and numerical methods, Mon. Not. R. Astron. Soc., 317, 550, arXiv:astro-ph/0005310 [astro-ph], 2000. (Cited on pages 91, 92, and 118.)

Porth, O., Olivares, H., Mizuno, Y., et al., The black hole accretion code, Computational Astrophysics and Cosmology, 4, 1, arXiv:1611.09720 [gr-qc], 2017. (Cited on pages $23,86,96,122$, and 176 .)

Porth, O., Xia, C., Hendrix, T., Moschou, S. P., \& Keppens, R., MPI-AMRVAC for Solar and Astrophysics, Astrophys. J., Supp., 214, 4, arXiv:1407.2052 [astro-ph.IM], 2014. (Cited on page 86.)

Porth, O., Chatterjee, K., Narayan, R., et al., The Event Horizon General Relativistic Magnetohydrodynamic Code Comparison Project, Astrophys. J. Supp., 243, 26, arXiv:1904.04923 [astro-ph.HE], 2019. (Cited on page 122.)

Punturo, M., Et sensitivities page, http://www.etgw.eu/index.php/etsensitivities, 2020. (Cited on pages 71 and 128.)

Punturo, M., et al., The third generation of gravitational wave observatories and their science reach, Class. Quantum Grav., 27, 084007, 2010. (Cited on pages 72, 128, and 175.)

Raaijmakers, G., Riley, T. E., Watts, A. L., et al., A Nicer View of PSR J0030+0451: Implications for the Dense Matter Equation of State, Astrophys. J. Lett., 887, L22, arXiv:1912.05703 [astro-ph.HE], 2019. (Cited on pages 57, 58, and 173.)

Radice, D., Binary Neutron Star Merger Simulations with a Calibrated Turbulence Model, arXiv e-prints, arXiv:2005.09002, arXiv:2005.09002 [astro-ph.HE], 2020. (Cited on page 73.) 
Radice, D., Abdikamalov, E., Rezzolla, L., \& Ott, C. D., A new spherical harmonics scheme for multi-dimensional radiation transport I. Static matter configurations, Journal of Computational Physics, 242, 648, arXiv:1209.1634 [astro-ph.HE], 2013. (Cited on pages 29 and 90.)

Radice, D., Galeazzi, F., Lippuner, J., et al., Dynamical Mass Ejection from Binary Neutron Star Mergers, Mon. Not. R. Astron. Soc., 460, 3255, arXiv:1601.02426 [astro-ph.HE], 2016. (Cited on page 30.)

Radice, D., Perego, A., Bernuzzi, S., \& Zhang, B., Long-lived remnants from binary neutron star mergers, Mon. Not. R. Astron. Soc., 481, 3670, arXiv:1803.10865 [astro-ph.HE], 2018. (Cited on page 64.)

Radice, D., Perego, A., Zappa, F., \& Bernuzzi, S., GW170817: Joint Constraint on the Neutron Star Equation of State from Multimessenger Observations, Astrophys. J. Lett., 852, L29, arXiv:1711.03647 [astro-ph.HE], 2018. (Cited on pages 49 and 50.)

Radice, D., \& Rezzolla, L., THC: a new high-order finite-difference high-resolution shock-capturing code for special-relativistic hydrodynamics, Astron. Astrophys., 547, A26, arXiv:1206.6502 [astro-ph.IM], 2012. (Cited on page 64.)

Radice, D., Rezzolla, L., \& Galeazzi, F., Beyond second-order convergence in simulations of binary neutron stars in full general-relativity, Mon. Not. R. Astron. Soc. L., 437, L46, arXiv:1306.6052 [gr-qc], 2014. (Cited on page 64.)

Radice, D., Rezzolla, L., \& Galeazzi, F., High-order fully general-relativistic hydrodynamics: new approaches and tests, Class. Quantum Grav., 31, 075012, arXiv:1312.5004 [gr-qc], 2014. (Cited on page 64.)

Rahman, N., Just, O., \& Janka, H. T., NADA-FLD: a general relativistic, multidimensional neutrino-hydrodynamics code employing flux-limited diffusion, Mon. Not. R. Astron. Soc., 490, 3545, arXiv:1901.10523 [astro-ph.HE], 2019. (Cited on page 30.)

Raithel, C., Özel, F., \& Psaltis, D., Tidal Deformability from GW170817 as a Direct Probe of the Neutron Star Radius, Astrophys. J., 857, L23, arXiv:1803.07687 [astroph.HE], 2018. (Cited on page 50.)

Raithel, C. A., Constraints on the neutron star equation of state from GW170817, European Physical Journal A, 55, 80, arXiv:1904.10002 [astro-ph.HE], 2019. (Cited on page 51.)

Raithel, C. A., Özel, F., \& Psaltis, D., From Neutron Star Observables to the Equation of State. I. An Optimal Parametrization, Astrophys. J., 831, 44, arXiv:1605.03591 [astro-ph.HE], 2016. (Cited on page 38.)

Raithel, C. A., Özel, F., \& Psaltis, D., From Neutron Star Observables to the Equation of State. II. Bayesian Inference of Equation of State Pressures, Astrophys. J., 844, 156, arXiv:1704.00737 [astro-ph.HE], 2017. (Cited on pages 35, 51, and 54.)

Rampp, M., Radiation Hydrodynamics with Neutrinos: Stellar Core Collapse and the Explosion Mechanism of Type II Supernovae, $\mathrm{PhD}$ thesis, Technische Universität München, 2000. (Cited on pages 27, 34, 78, 106, and 112.)

Read, J. S., Lackey, B. D., Owen, B. J., \& Friedman, J. L., Constraints on a phenomenologically parametrized neutron-star equation of state, Phys. Rev. D, 79, 124032, arXiv:0812.2163, 2009. (Cited on pages 35, 38, and 51.) 
Reisswig, C., \& Pollney, D., Notes on the integration of numerical relativity waveforms, Class. Quantum Grav., 28, 195015, arXiv:1006.1632 [gr-qc], 2011. (Cited on page 20.)

Rezzolla, L., Baiotti, L., Giacomazzo, B., Link, D., \& Font, J. A., Accurate evolutions of unequal-mass neutron-star binaries: properties of the torus and short GRB engines, Class. Quantum Grav., 27, 114105, arXiv:1001.3074 [gr-qc], 2010. (Cited on page 45.)

Rezzolla, L., Giacomazzo, B., Baiotti, L., et al., The Missing Link: Merging Neutron Stars Naturally Produce Jet-like Structures and Can Power Short Gamma-ray Bursts, Astrophys. J. Letters, 732, L6, arXiv:1101.4298 [astro-ph.HE], 2011. (Cited on pages $5,45,122$, and 172 .)

Rezzolla, L., \& Miller, J. C., Relativistic radiative transfer for spherical flows, Class. Quantum Grav., 11, 1815, arXiv:arXiv:astro-ph/9406055, 1994. (Cited on pages 30 and 78.)

Rezzolla, L., Most, E. R., \& Weih, L. R., Using Gravitational-wave Observations and Quasi-universal Relations to Constrain the Maximum Mass of Neutron Stars, Astrophys. J. Lett., 852, L25, arXiv:1711.00314 [astro-ph.HE], 2018. (Cited on pages 35, $45,46,48,52,127$, and 172.)

Rezzolla, L., Pizzochero, P., Jones, D. I., Rea, N., \& Vidaña, I., The Physics and Astrophysics of Neutron Stars, 2018. (Cited on page 1.)

Rezzolla, L., \& Takami, K., Gravitational-wave signal from binary neutron stars: A systematic analysis of the spectral properties, Phys. Rev. D, 93, 124051, arXiv:1604.00246 [gr-qc], 2016. (Cited on pages 5, 20, 62, 70, and 175.)

Rezzolla, L., \& Zanotti, O., Relativistic hydrodynamics (Oxford, UK: Oxford University Press), 2013. (Cited on pages 22, 23, 25, 36, 40, 64, and 114.)

Richers, S., Nagakura, H., Ott, C. D., et al., A Detailed Comparison of Multidimensional Boltzmann Neutrino Transport Methods in Core-collapse Supernovae, Astrophys. J., 847, 133, arXiv:1706.06187 [astro-ph.HE], 2017. (Cited on page 31.)

Riffert, H., A General Eulerian Formulation of the Comoving-Frame Equation of Radiative Transfer, Astrophys. J., 310, 729, 1986. (Cited on page 28.)

Riley, T. E., Watts, A. L., Bogdanov, S., et al., A NICER View of PSR J0030+0451: Millisecond Pulsar Parameter Estimation, Astrophys. J. Lett., 887, L21, arXiv:1912.05702 [astro-ph.HE], 2019. (Cited on pages 51, 57, 58, 173, and 174.)

Ripperda, B., Bacchini, F., Porth, O., et al., General-relativistic Resistive Magnetohydrodynamics with Robust Primitive-variable Recovery for Accretion Disk Simulations, Astrophys. J., Supp., 244, 10, arXiv:1907.07197 [physics.comp-ph], 2019. (Cited on pages 25, 82, and 85.)

Rivera-Paleo, F. J., \& Guzmán, F. S., CAFE-R: A Code That Solves the Special Relativistic Radiation Hydrodynamics Equations, Astrophys. J., Supp., 241, 28, arXiv:1903.04994 [astro-ph.HE], 2019. (Cited on pages 88, 114, and 122.)

Rivet, J.-P., \& Boon, J. P., Lattice gas hydrodynamics (Cambridge University Press), 2001. (Cited on page 108.) 
Roedig, C., Zanotti, O., \& Alic, D., General relativistic radiation hydrodynamics of accretion flows - II. Treating stiff source terms and exploring physical limitations, Mon. Not. R. Astron. Soc., 426, 1613, arXiv:1206.6662 [astro-ph.HE], 2012. (Cited on pages $30,75,80,85,94,95,96,98,125$, and 175 .)

Romero-Shaw, I. M., Farrow, N., Stevenson, S., Thrane, E., \& Zhu, X.-J., On the origin of GW190425, arXiv e-prints, arXiv:2001.06492, arXiv:2001.06492 [astroph.HE], 2020. (Cited on pages 59 and 129.)

Rosswog, S., Mergers of neutron star black hole binaries with small mass ratios: nucleosynthesis, gamma-ray bursts and electromagnetic transients, Astrophys. J., 634, 1202, arXiv:astro-ph/0508138, 2005. (Cited on pages 6, 32, and 74.)

Rosswog, S., Feindt, U., Korobkin, O., et al., Detectability of compact binary merger macronovae, Classical and Quantum Gravity, 34, 104001, arXiv:1611.09822 [astroph.HE], 2017. (Cited on page 5.)

Rosswog, S., \& Liebendörfer, M., High-resolution calculations of merging neutron stars - II. Neutrino emission, Mon. Not. R. Astron. Soc., 342, 673, arXiv:arXiv:astro-ph/0302301, 2003. (Cited on pages 29, 34, and 125.)

Rosswog, S., Liebendörfer, M., Thielemann, F.-K., et al., Mass ejection in neutron star mergers, Astron. Astrophys., 341, 499, arXiv:astro-ph/9811367, 1999. (Cited on page 5.)

Ruffert, M., Janka, H.-T., \& Schaefer, G., Coalescing neutron stars - a step towards physical models. I. Hydrodynamic evolution and gravitational-wave emission., Astron. Astrophys., 311, 532, arXiv:astro-ph/9509006, 1996. (Cited on pages 29, 34, and 125.)

Ruiz, M., Paschalidis, V., Tsokaros, A., \& Shapiro, S. L., Black hole-neutron star coalescence: effects of the neutron star spin on jet launching and dynamical ejecta mass, arXiv e-prints, arXiv:2011.08863, arXiv:2011.08863 [astro-ph.HE], 2020. (Cited on page 128.)

Ruiz, M., Shapiro, S. L., \& Tsokaros, A., GW170817, general relativistic magnetohydrodynamic simulations, and the neutron star maximum mass, Phys. Rev. D, 97, 021501, arXiv:1711.00473 [astro-ph.HE], 2018. (Cited on pages 48, 52, 127, and 173.)

Ruiz, M., Shapiro, S. L., \& Tsokaros, A., Jet launching from binary black hole-neutron star mergers: Dependence on black hole spin, binary mass ratio, and magnetic field orientation, Phys. Rev. D, 98, 123017, arXiv:1810.08618 [astro-ph.HE], 2018. (Cited on page 128.)

Ruiz, M., Tsokaros, A., Shapiro, S. L., Nelli, K. C., \& Qunell, S., Magnetic ergostars, jet formation, and gamma-ray bursts: Ergoregions versus horizons, Phys. Rev. D, 102, 104022, arXiv:2009.08982 [astro-ph.HE], 2020. (Cited on page 128.)

Ryan, B. R., Dolence, J. C., \& Gammie, C. F., bhlight: General Relativistic Radiation Magnetohydrodynamics with Monte Carlo Transport, Astophys. J., 807, 31, arXiv:1505.05119 [astro-ph.HE], 2015. (Cited on page 31.)

Rybicki, G. B., \& Lightman, A. P., Radiative Processes in Astrophysics (Wiley-VCH), 1986. (Cited on pages 34, 96, and 123.) 
Safarzadeh, M., Ramirez-Ruiz, E., \& Berger, E., GW190425 is inconsistent with being a binary neutron star born from a fast merging channel, arXiv e-prints, arXiv:2001.04502, arXiv:2001.04502 [astro-ph.HE], 2020. (Cited on pages 59 and 129.)

Sakuragi, Y., Saturation of Nuclear Matter and Roles of Many-Body Forces: nuclear matter in neutron stars probed by nucleus-nucleus scattering, arXiv e-prints, arXiv:1604.07632, arXiv:1604.07632 [nucl-th], 2016. (Cited on page 37.)

Savchenko, V., Ferrigno, C., Kuulkers, E., et al., INTEGRAL Detection of the First Prompt Gamma-Ray Signal Coincident with the Gravitational-wave Event GW170817, Astrophys. J. Letters, 848, L15, arXiv:1710.05449 [astro-ph.HE], 2017. (Cited on page 45.)

Sądowski, A., Narayan, R., Tchekhovskoy, A., \& Zhu, Y., Semi-implicit scheme for treating radiation under $M 1$ closure in general relativistic conservative fluid dynamics codes, Mon. Not. R. Astron. Soc., 429, 3533, arXiv:1212.5050 [astro-ph.HE], 2013. (Cited on pages $30,75,85,94,96,97,98,125$, and 175.)

Schaffner-Bielich, J., Compact star physics (Cambridge, UK: Cambridge University Press), 2020. (Cited on pages 1 and 2.)

Schnetter, E., Hawley, S. H., \& Hawke, I., Evolutions in 3D numerical relativity using fixed mesh refinement, Class. Quantum Grav., 21, 1465, arXiv:gr-qc/0310042, 2004. (Cited on page 65.)

Schwarzschild, K., Über das Gravitationsfeld eines Massenpunktes nach der Einsteinschen Theorie, Sitzungsberichte der Königlich Preußischen Akademie der Wissenschaften (Berlin, 189, 1916. (Cited on page 12.)

Sedrakian, A., Weber, F., \& LI, J.-J., Confronting GW190814 with hyperonization in dense matter and hypernuclear compact stars, arXiv e-prints, arXiv:2007.09683, arXiv:2007.09683 [astro-ph.HE], 2020. (Cited on page 59.)

Sekiguchi, Y., An implementation of the microphysics in full general relativity: a general relativistic neutrino leakage scheme, Class. Quantum Grav., 27, 114107, arXiv:1009.3358 [astro-ph.HE], 2010. (Cited on page 29.)

Sekiguchi, Y., Kiuchi, K., Kyutoku, K., Shibata, M., \& Taniguchi, K., Dynamical mass ejection from the merger of asymmetric binary neutron stars: Radiation-hydrodynamics study in general relativity, Phys. Rev. D, 93, 124046, arXiv:1603.01918 [astro-ph.HE], 2016. (Cited on pages 30, 45, 75, and 175.)

Shan, X., The mathematical structure of the lattices of the lattice boltzmann method, Journal of Computational Science, 17, 475 , 2016. (Cited on page 107.)

Shan, X., \& He, X., Discretization of the velocity space in the solution of the boltzmann equation, Phys. Rev. Lett., 80, 65, 1998. (Cited on page 103.)

Shan, X., Yuan, X.-F., \& Chen, H., Kinetic theory representation of hydrodynamics: a way beyond the navier-stokes equation, Journal of Fluid Mechanics, 550, 413, 2006. (Cited on page 107.)

Shao, D.-S., Tang, S.-P., Jiang, J.-L., \& Fan, Y.-Z., Maximum mass cutoff in the neutron star mass distribution and the prospect of forming supramassive objects in the double neutron star mergers, Phys. Rev. D, 102, 063006, arXiv:2009.04275 [astro-ph.HE], 2020. (Cited on page 47.) 
Shapiro, I. I., Fourth Test of General Relativity, Physical Review Letters, 13, 789, 1964. (Cited on page 3.)

Shapiro, S. L., Radiation from Stellar Collapse to a Black Hole, Astrophys. J., 472, 308, 1996. (Cited on page 34.)

Shapiro, S. L., Differential Rotation in Neutron Stars: Magnetic Braking and Viscous Damping, Astrophys. J., 544, 397, arXiv:astro-ph/0010493 [astro-ph], 2000. (Cited on page 46.)

Shapiro, S. L., \& Teukolsky, S. A., Black holes, white dwarfs, and neutron stars (New York: John Wiley \& Sons), 1983. (Cited on page 36.)

Shet, A. G., Sorathiya, S. H., Krithivasan, S., et al., Data structure and movement for lattice-based simulations, Phys. Rev. E, 88, 013314, 2013. (Cited on page 120.)

Shibata, M., Numerical Relativity (Singapore: World Scientific), 2016. (Cited on page 10.)

Shibata, M., Fujibayashi, S., Hotokezaka, K., et al., Modeling GW170817 based on numerical relativity and its implications, Phys. Rev. D, 96, 123012, arXiv:1710.07579 [astro-ph.HE], 2017. (Cited on pages 47, 48, 52, 127, and 173.)

Shibata, M., Kiuchi, K., Sekiguchi, Y., \& Suwa, Y., Truncated Moment Formalism for Radiation Hydrodynamics in Numerical Relativity, Progress of Theoretical Physics, 125, 1255, arXiv:1104.3937 [astro-ph.HE], 2011. (Cited on pages 27, 28, 34, 76, 77, 78, $79,98,106$, and 176.)

Shibata, M., Kiuchi, K., \& Sekiguchi, Y.-i., General relativistic viscous hydrodynamics of differentially rotating neutron stars, Phys. Rev. D, 95, 083005, 2017. (Cited on page 4.)

Shibata, M., \& Nakamura, T., Evolution of three-dimensional gravitational waves: Harmonic slicing case, Phys. Rev. D, 52, 5428, 1995. (Cited on page 15.)

Shibata, M., Zhou, E., Kiuchi, K., \& Fujibayashi, S., Constraint on the maximum mass of neutron stars using GW170817 event, Phys. Rev. D, 100, 023015, arXiv:1905.03656 [astro-ph.HE], 2019. (Cited on pages 47, 48, 127, and 173.)

Siegel, D. M., Ciolfi, R., \& Rezzolla, L., Magnetically Driven Winds from Differentially Rotating Neutron Stars and X-Ray Afterglows of Short Gamma-Ray Bursts, Astrophys. J., 785, L6, arXiv:1401.4544 [astro-ph.HE], 2014. (Cited on page 45.)

Skinner, M. A., Dolence, J. C., Burrows, A., Radice, D., \& Vartanyan, D., FORNAX: A Flexible Code for Multiphysics Astrophysical Simulations, Astrophys. J., Supp., 241, 7, arXiv:1806.07390 [astro-ph.IM], 2019. (Cited on pages 30, 75, and 175.)

Smit, J. M., Cernohorsky, J., \& Dullemond, C. P., Hyperbolicity and critical points in two-moment approximate radiative transfer., Astron. Astrophys., 325, 203, 1997. (Cited on pages 89 and 117.)

Sobolev, S., Cubature formulas on the sphere invariant under finite groups of rotations, Soviet Mathematics Doklady, 3, 1307, 1962. (Cited on page 110.)

Steiner, A. W., Lattimer, J. M., \& Brown, E. F., The Equation of State from Observed Masses and Radii of Neutron Stars, Astrophys. Journ., 722, 33, arXiv:1005.0811 [astro-ph.HE], 2010. (Cited on pages 35 and 51.) 
Stepán, Jirí, Bestard, Jaume Jaume, \& Trujillo Bueno, Javier, Near optimal angular quadratures for polarised radiative transfer, A\&A, 636, A24, 2020. (Cited on page 110.)

Stergioulas, N., \& Friedman, J. L., Comparing models of rapidly rotating relativistic stars constructed by two numerical methods, Astrophys. J., 444, 306, arXiv:astroph/9411032, 1995. (Cited on page 43.)

Studzińska, A. M., Kucaba, M., Gondek-Rosińska, D., Villain, L., \& Ansorg, M., Effect of the equation of state on the maximum mass of differentially rotating neutron stars, Mon. Not. R. Astron. Soc., 463, 2667, 2016. (Cited on page 44.)

Succi, S., Lattice Boltzmann across scales: from turbulence to DNA translocation, European Physical Journal B, 64, 471, 2008. (Cited on page 102.)

Succi, S., The Lattice Boltzmann Equation: For Complex States of Flowing Matter (OUP Oxford), 2018. (Cited on pages 32, 102, and 176.)

Succi, S., Amati, G., Bernaschi, M., et al., Towards exascale lattice boltzmann computing, Computers \& Fluids, 181, 107 , 2019. (Cited on page 120.)

Succi, S., Sbragaglia, M., \& Ubertini, S., Lattice Boltzmann Method, Scholarpedia, 5, 9507, 2010. (Cited on pages 102 and 176.)

Sumiyoshi, K., Fujibayashi, S., Sekiguchi, Y., \& Shibata, M., Properties of neutrino transfer in a deformed remnant of neutron star merger, arXiv e-prints, arXiv:2010.10865, arXiv:2010.10865 [astro-ph.HE], 2020. (Cited on pages 80 and 102.)

Suresh, A., \& Huynh, H. T., Accurate monotonicity-preserving schemes with rungekutta time stepping, Journal of Computational Physics, 136, 83, 1997. (Cited on page 64.)

Takami, K., Rezzolla, L., \& Baiotti, L., Constraining the Equation of State of Neutron Stars from Binary Mergers, Phys. Rev. Lett., 113, 091104, arXiv:1403.5672 [gr-qc], 2014. (Cited on pages 5, 62, 70, 71, and 175.)

Takami, K., Rezzolla, L., \& Baiotti, L., Spectral properties of the post-merger gravitational-wave signal from binary neutron stars, Phys. Rev. D, 91, 064001, arXiv:1412.3240 [gr-qc], 2015. (Cited on pages 5, 62, 70, and 175.)

Takami, K., Rezzolla, L., \& Yoshida, S., A quasi-radial stability criterion for rotating relativistic stars, Mon. Not. R. Astron. Soc., 416, L1, arXiv:1105.3069 [gr-qc], 2011. (Cited on pages 5, 44, 46, and 172.)

Tan, H., Noronha-Hostler, J., \& Yunes, N., Kinky neutron stars in light of GW190814, arXiv e-prints, arXiv:2006.16296, arXiv:2006.16296 [astro-ph.HE], 2020. (Cited on page 59.)

Tanaka, M., Kato, D., Gaigalas, G., et al., Properties of Kilonovae from Dynamical and Post-merger Ejecta of Neutron Star Mergers, Astrophys. J., 852, 109, arXiv:1708.09101 [astro-ph.HE], 2018. (Cited on page 5.)

Tanvir, N. R., Levan, A. J., González-Fernández, C., et al., The Emergence of a Lanthanide-rich Kilonova Following the Merger of Two Neutron Stars, Astrophys. J. Letters, 848, L27, arXiv:1710.05455 [astro-ph.HE], 2017. (Cited on page 5.) 
Tews, I., Margueron, J., \& Reddy, S., Critical examination of constraints on the equation of state of dense matter obtained from GW170817, Physical Review C, 98, 045804, arXiv:1804.02783 [nucl-th], 2018. (Cited on pages 38 and 50.)

Tews, I., Pang, P. T. H., Dietrich, T., et al., On the nature of GW190814 and its impact on the understanding of supranuclear matter, arXiv e-prints, arXiv:2007.06057, arXiv:2007.06057 [astro-ph.HE], 2020. (Cited on page 38.)

The LIGO Scientific Collaboration, \& The Virgo Collaboration, Gw170817: Observation of gravitational waves from a binary neutron star inspiral, Phys. Rev. Lett., 119, 161101, arXiv:1710.05832 [gr-qc], 2017. (Cited on page 38.)

The LIGO Scientific Collaboration, \& the Virgo Collaboration, Gwtc-2: an expanded catalog of gravitational-wave detections, https://www.ligo.org/science/Publication-03aCatalog/, 2020. (Cited on pages 129 and 177.)

The LIGO Scientific Collaboration, the Virgo Collaboration, Abbott, B. P., et al., Properties of the Binary Neutron Star Merger GW170817, Physical Review X, 9, 011001, arXiv:1805.11579 [gr-qc], 2019. (Cited on page 52.)

The LIGO Scientific Collaboration, the Virgo Collaboration, Abbott, R., et al., GW190814: Gravitational Waves from the Coalescence of a 23 Solar Mass Black Hole with a 2.6 Solar Mass Compact Object, Astrophys. J. Lett., 896, L44, arXiv:2006.12611 [astro-ph.HE], 2020. (Cited on page 59.)

Thorne, K. S., Relativistic radiative transfer - Moment formalisms, Mon. Not. R. Astron. Soc., 194, 439, 1981. (Cited on pages 30, 76, 78, and 175.)

Timmes, F. X., Woosley, S. E., \& Weaver, T. A., The Neutron Star and Black Hole Initial Mass Function, Astrophys. J., 457, 834, arXiv:astro-ph/9510136, 1996. (Cited on pages 47 and 48 .)

Tolman, R. C., Static Solutions of Einstein's Field Equations for Spheres of Fluid, Physical Review, 55, 364, 1939. (Cited on page 36.)

Tolos, L., Centelles, M., \& Ramos, A., Equationn of State for Nucleonic and Hyperonic Neutron Stars with Mass and Radius Constraints, Astrophys. J., 834, 3, arXiv:1610.00919 [astro-ph.HE], 2017. (Cited on pages 2, 63, and 174.)

Tolos, L., Centelles, M., \& Ramos, A., The Equation of State for the Nucleonic and Hyperonic Core of Neutron Stars, Publications of the Astronomical Society of Australia, 34, e065, arXiv:1708.08681 [astro-ph.HE], 2017. (Cited on pages 63 and 174.)

Toro, E. F., Riemann solvers and numerical methods for fluid dynamics (SpringerVerlag), 2009. (Cited on page 24.)

Trangenstein, J., Numerical solution of hyperbolic partial differential equations (Cambridge University Press), 2009. (Cited on page 24.)

Turner, N. J., \& Stone, J. M., A Module for Radiation Hydrodynamic Calculations with ZEUS-2D Using Flux-limited Diffusion, Astrophys. J., Supp., 135, 95, arXiv:astro-ph/0102145 [astro-ph], 2001. (Cited on page 93.)

Typel, S., Röpke, G., Klähn, T., Blaschke, D., \& Wolter, H. H., Composition and thermodynamics of nuclear matter with light clusters, Phys. Rev. C, 81, 015803, arXiv:0908.2344 [nucl-th], 2010. (Cited on page 47.) 
Usov, V. V., Millisecond pulsars with extremely strong magnetic fields as a cosmological source of gamma-ray bursts, Nature, 357, 472, 1992. (Cited on page 46.)

van Leer, B., Towards the ultimate conservative difference scheme. v. a second-order sequel to godunov's method, Journal of Computational Physics, 32, 101, 1979. (Cited on page 24.)

Vernekar, R. R., \& Mishra, S. C., Analysis of transport of short-pulse radiation in a participating medium using lattice boltzmann method, International Journal of Heat and Mass Transfer, 77, 218 , 2014. (Cited on page 105.)

Villar, V. A., Guillochon, J., Berger, E., et al., The Combined Ultraviolet, Optical, and Near-infrared Light Curves of the Kilonova Associated with the Binary Neutron Star Merger GW170817: Unified Data Set, Analytic Models, and Physical Implications, Astrophys. J. Letters, 851, L21, arXiv:1710.11576 [astro-ph.HE], 2017. (Cited on pages 5,45 , and 172 .)

Vitello, P. A. J., Spherically symmetric accretion near the Eddington limit, Astrop. J., 225, 694, 1978. (Cited on page 94.)

Waters, T., Aykutalp, A., Proga, D., et al., Outflows from inflows: the nature of Bondi-like accretion, Mon. Not. R. Astron. Soc., 491, L76, arXiv:1910.01106 [astroph.GA], 2020. (Cited on page 99.)

Watts, A. L., Andersson, N., Chakrabarty, D., et al., Colloquium: Measuring the neutron star equation of state using x-ray timing, Reviews of Modern Physics, 88, 021001, arXiv:1602.01081 [astro-ph.HE], 2016. (Cited on pages 51 and 53.)

Weber, F., Farrell, D., Spinella, W. M., et al., Phases of Hadron-Quark Matter in (Proto) Neutron Stars, Universe, 5, 169, arXiv:1907.06591 [nucl-th], 2019. (Cited on page 63.)

Weih, L. R., Gabbana, A., Simeoni, D., et al., Beyond moments: relativistic lattice Boltzmann methods for radiative transport in computational astrophysics, Mon. Not. R. Astron. Soc., 498, 3374, arXiv:2007.05718 [physics.comp-ph], 2020. (Cited on pages $32,75,102,105,109,111,114,115,116,117,118,119,120,121,123,124,128$, and 137.)

Weih, L. R., Hanauske, M., \& Rezzolla, L., Postmerger Gravitational-Wave Signatures of Phase Transitions in Binary Mergers, Phys. Rev. Lett., 124, 171103, arXiv:1912.09340 [gr-qc], 2020. (Cited on pages 60, 61, 62, 64, 66, 69, 70, 71, 72, 127, and 174.)

Weih, L. R., Most, E. R., \& Rezzolla, L., On the stability and maximum mass of differentially rotating relativistic stars, Mon. Not. R. Astron. Soc., 473, L126, arXiv:1709.06058 [gr-qc], 2018. (Cited on pages 5, 44, 46, and 172.)

Weih, L. R., Most, E. R., \& Rezzolla, L., Animated Figure (Zenodo), 2019. (Cited on pages 52,53 , and 55 .)

Weih, L. R., Most, E. R., \& Rezzolla, L., EOS library (Zenodo), 2019. (Cited on pages $52,63,127,173$, and 174 .)

Weih, L. R., Most, E. R., \& Rezzolla, L., Optimal Neutron-star Mass Ranges to Constrain the Equation of State of Nuclear Matter with Electromagnetic and Gravitational-wave Observations, Astrophys. J., 881, 73, arXiv:1905.04900 [astroph.HE], 2019. (Cited on pages 2, 35, 38, 40, 51, 52, 54, 55, 56, 57, 58, 127, and 173.) 
Weih, L. R., Olivares, H., \& Rezzolla, L., Two-moment scheme for general-relativistic radiation hydrodynamics: a systematic description and new applications, Mon. Not. R. Astron. Soc., 495, 2285, arXiv:2003.13580 [gr-qc], 2020. (Cited on pages 30, 34, 75, $86,87,88,89,90,91,92,93,95,96,98,99,100,101,114,128$, and 176.)

Weinberg, S., Gravitation and cosmology: Principles and applications of the general theory of relativity (New York: John Wiley and Sons), 1972. (Cited on pages 10 and 36.)

Womersley, R. S., in Contemporary Computational Mathematics - A Celebration of the 80th Birthday of Ian SloanEfficient spherical designs with good geometric properties, (Springer International Publishing) 1243, 2018. (Cited on page 111.)

Yamada, S., Janka, H.-T., \& Suzuki, H., Neutrino transport in type II supernovae: Boltzmann solver vs. Monte Carlo method, Astron. Astrophys., 344, 533, arXiv:astro-ph/9809009 [astro-ph], 1999. (Cited on page 32.)

Yi, H.-L., Yao, F.-J., \& Tan, H.-P., Lattice boltzmann model for a steady radiative transfer equation, Phys. Rev. E, 94, 023312, 2016. (Cited on page 105.)

Zampieri, L., Miller, J. C., \& Turolla, R., Time-dependent analysis of spherical accretion on to black holes, Mon. Not. R. Astron. Soc., 281, 1183, arXiv:arXiv:astroph/9607030, 1996. (Cited on page 94.)

Zanotti, O., Roedig, C., Rezzolla, L., \& Del Zanna, L., General relativistic radiation hydrodynamics of accretion flows - I. Bondi-Hoyle accretion, Mon. Not. R. Astron. Soc., 417, 2899, arXiv:1105.5615 [astro-ph.HE], 2011. (Cited on pages 30, 75, 95, and 175.)

Zhang, B., \& Mészáros, P., Gamma-Ray Burst Afterglow with Continuous Energy Injection: Signature of a Highly Magnetized Millisecond Pulsar, Astrophys. J., 552, L35, arXiv:astro-ph/0011133, 2001. (Cited on page 46.)

Zhang, N.-B., \& Li, B.-A., GW190814's secondary component with mass (2.50 2.67) $M_{\odot}$ as a super-fast pulsar, arXiv e-prints, arXiv:2007.02513, arXiv:2007.02513 [astro-ph.HE], 2020. (Cited on page 59.)

Zhu, X., Thrane, E., Osłowski, S., Levin, Y., \& Lasky, P. D., Inferring the population properties of binary neutron stars with gravitational-wave measurements of spin, Phys. Rev. D, 98, 043002, arXiv:1711.09226 [astro-ph.HE], 2018. (Cited on page 44.)

Zlochower, Y., Nakano, H., Mundim, B. C., et al., Inspiraling black-hole binary spacetimes: Challenges in transitioning from analytical to numerical techniques, Phys. Rev. D, 93, 124072, arXiv:1504.00286 [gr-qc], 2016. (Cited on page 15.) 


\section{Zusammenfassung}

Im August 2017 hat das LIGO und Virgo Netzwerk (LVC) zum ersten Mal Gravitationswellen von einem verschmelzendem Neutronenstern-Binärsystem detektiert (Abbott et al., 2017). Diese Entdeckung wurde begleitet von einem kurzen Gammablitz (engl. short gamma-ray burst; SGRB), der 1.7 Sekunden später entdeckt wurde (LIGO Scientific Collaboration et al., 2017). Durch die präzise Lokalisation dieser Signale, konnten Teleskope den Ursprung finden und beobachteten ein helles Leuchten im ultravioletten, optischen und infraroten Spektrum (Drout et al., 2017; Villar et al., 2017; Cowperthwaite et al., 2017). Dieses Leuchten wird als Kilonova bezeichnet und entsteht durch den radioaktiven Zerfall der Elemente, die in dem neutronenreichen Massenauswurf der verschmelzenden Sterne gebildet werden (Just et al., 2015a). Dieses Event, welches als GW170817 bezeichnet wird, wird als Beginn der Multimessenger-Ära angesehen (Metzger, 2017b). Gleichzeitig fällt der Zeitpunkt dieses Events auch mit dem Beginn der vorliegenden Arbeit zusammen.

Nach einer kurzen Zusammenfassung der relevanten Gleichungen in Kap. 2, werden die Ergebnisse, die im Zusammenhang mit der vorliegenden Dissertation erarbeitet wurden, in drei Kapiteln (Kap. 3-5) präsentiert.

\section{Kapitel 3: Der Einfluss von GW170817 auf die Zustandsgleichung von Kernmaterie}

Chronologisch beginnt diese Arbeit mit der Aufarbeitung der vielen Daten und Informationen, die von GW170817 gewonnen wurden. In Kap. 3 werden zunächst die Ergebnisse aus Rezzolla et al. (2018a) (s. Abschn. 1.3 für die Beiträge des Autors zu den Publikation innerhalb dieser Dissertation) präsentiert.

Hierbei werden die Messung der Gravitationswellen und die Beobachtung der Kilonova genutzt, um eine obere Grenze für die Maximalmasse von nicht-rotierenden Neutronensternen, $M_{\text {то }}$, herzuleiten. Ausgehend von der gemessenen Masse des Binärsystems, $M_{\text {tot }} \sim 2.74 M_{\odot}$, ergeben sich zwei mögliche Trajektorien für die Bewegung des Verschmelzungsprodukts, des hypermassiven Neutronensterns (HMNS), $\operatorname{im} M-\rho_{\mathrm{c}}$ Raum (s. Abb. 3.3). Aufgrund der Signatur der Kilonova wird vermutet, dass der HMNS nicht direkt zu einem schwarzen Loch kollabierte, sondern zumindest lange genug überlebte, um von einem differentiell zu einem gleichmäßig rotierenden Neutronenstern zu werden. Andererseits hat der HMNS nicht länger als $\sim 1.7$ Sekunden überlebt, da die Beobachtung des SGRBs den meisten Modellen zufolge (s. z.B. Rezzolla et al., 2011) den Kollaps zu einem schwarzen Loch voraussetzt. Unter diesen Annahmen ist es nun möglich die Masse des Neutronensterns zum Zeitpunkt des Kollapses herzuleiten. Gleichzeitig zeigen theoretische Modelle (Takami et al., 2011; Weih et al., 2018), dass es ein strenges Stabilitätskriterium für diesen Kollaps gibt (s. Abb. 3.2) und dass die Masse an dieser Stabilitätsgrenze über eine universelle Beziehung mit der zugrundeliegenden Zustandsgleichung verbunden werden kann (Breu \& Rezzolla, 2016). Insgesamt ergibt sich dann folgende Relation für die obere Grenze der maximalen Neutronenstermasse

$$
M_{\mathrm{TOV}}<\chi^{-1}\left(\xi M_{\mathrm{tot}}-\eta^{-1} M_{\mathrm{ej}}\right),
$$

wobei $\chi$ der universelle Umrechnungsfaktor zwischen den Massen rotierender und nicht-rotierender Neutronensterne ist (Breu \& Rezzolla, 2016), $\xi$ der Anteil der Masse im Kern des ursprünglichen HMNS (Hanauske et al., 2017a), $\eta$ der Umrechnungsfaktor 
zwischen Baryon- und Gravitationsmasse (s. Abb. 3.4) und $M_{\mathrm{ej}}$ die während der Verschmelzung abgeworfene Masse (Cowperthwaite et al., 2017; Drout et al., 2017). Setzt man die entsprechende Werte ein, erhält man

$$
M_{\text {TOV }}<2.16_{-0.15}^{+0.17} M_{\odot},
$$

wobei die Fehler der zweifachen Standardabweichung entsprechen, die durch Gauß'sche Fehlerfortpflanzung berechnet wurde. Dieses Ergebnis wurde innerhalb kürzester Zeit unabhängig von drei weiteren Arbeitsgruppen, die jeweils unterschiedliche Methoden benutzten, bestätigt (Margalit \& Metzger, 2017; Ruiz et al., 2018a; Shibata et al., 2017). Insgesamt wird die obere Grenze von $M_{\text {то }} \lesssim 2.3 M_{\odot}$ als robust angesehen (Shibata et al., 2019).

Neben diesem Wert, konnte auch die Größe von Neutronensternen eingeschränkt werden. Genauer gesagt wurde der Radius mithilfe der gemessenen Gezeitenverformbarkeit, $\tilde{\Lambda}$ (s. Abschn. 3.1.2), eingegrenzt. Die Gravitationswellenmessung zeigt, dass die beiden Sterne durch die Gezeitenkräfte, die durch das Gravitationsfeld des jeweiligen Partners entstehen, verformt werden. Der Wert hierfür wurde ursprünglich gemessen als $\tilde{\Lambda}<800$ (Abbott et al., 2017). Durch das erweiterte System der TOV-Gleichungen (s. Abschn. 3.1.1) kann diese Größe mit dem Radius eines Neutronensterns mit einer Masse von 1.4, $M_{\odot}$ in Verbindung gebracht werden. Mit einer parametrisierten Zustandsgleichung wurde in Most et al. (2018) ein Datensatz von mehreren Milliarden Neutronensternmodellen konstruiert. Zusammen mit dem oberen Limit für $M_{\mathrm{TOV}}$ und dem bekannten unterem Limit von $M_{\text {TоV }}>2.01 M_{\odot}$ (Antoniadis et al., 2013) ergibt sich

$$
12.00 \mathrm{~km}<R_{1.4}<13.45 \mathrm{~km} .
$$

Ein ähnlicher Wert wurde wiederum von vielen anderen Arbeitsgruppen hergeleitet und eine teilweise Zusammenfassung über alle Größen, die nach der Detektion von GW170817 hergeleitet wurden, ist in Tab. $3.1 \mathrm{zu}$ finden. Inwiefern sich all diese Einschränkungen für Neutronensterngrößen auf die Zustandsgleichung nuklearer Materie auswirkt, ist in Abb. 3.11 dargestellt.

Während der Anfertigung dieser Arbeit wurde unser Verständnis von Neutronensternen nicht nur durch Gravitationswellendetektionen, sondern auch durch direkte Beobachtungen, erweitert. Dazu kam es durch die Röntgenaufnahmen der NICER Mission, die im Dezember 2019 publiziert wurden (Riley et al., 2019; Raaijmakers et al., 2019; Bilous et al., 2019; Bogdanov et al., 2019a,b; Miller et al., 2019c; Guillot et al., 2019). Kurz vorher wurde bereits in Weih et al. (2019) analysiert wie solche direkten Radiusmessungen die Ergebnisse um GW170817 komplementieren werden. Durch denselben Datensatz, der bereits in Most et al. (2018) verwendet wurde und öffentlich verfügbar ist (Weih et al., 2019b), wurde analysiert wie jede mögliche MasseRadius-Beobachtung sich auf unser Verständnis über die Zustandsgleichung auswirkt. Hierzu ist die Größe

$$
\chi:=1-\frac{A}{A_{0}}
$$

definiert, die ein Maß dafür darstellt wie weit die eingenommene Fläche, $A$, aller möglichen Zustandsgleichungen im $p-\mu$ Raum durch eine Beobachtung eingeschränkt wird im Vergleich zur Referenzfläche $A_{0}$ (s. Abb. 3.6). Die Analyse von drei möglichen Szenarien, wobei für jedes Szenario jedes mögliche Ergebnis in Betracht gezogen wird, ergibt, dass die meiste Information aus einer Radiusmessung der schwersten Neutronensterne hergeleitet werden kann. Dies ist ein bekanntes Ergebnis, das allerdings zum ersten Mal quantifiziert wurde. Aus Abb. 3.9 wird außerdem ersichtlich, dass für 
den Teil der Zustandsgleichung mit niedrigerer Dichte (bis zu einem chemischen Potential von $\mu<1.12 \mathrm{GeV}$ ), welcher typischerweise durch chirale effektive Feldtheorie untersucht wird, auch Neutronensterne mit einer Masse von $1.8 M_{\odot}$ vielversprechende Kandidaten für zukünftige Beobachtungen darstellen.

Diese Ergebnisse sind unabhängig von den Beobachtungen von NICER und zeigen das Potential von zukünftigen Röntgenbeobachtungen. Dieselbe Methode kann ebenfalls genutzt werden, um den Einfluss von zukünftigen Gravitationswellenbeobachtungen zu analysieren. Außerdem hat die Analyse mit demselben Datensatz (Weih et al., 2019b) ergeben, dass die tatsächlichen Ergebnisse der NICER Mission (Riley et al., 2019; Miller et al., 2019c) die Zustandsgleichung im Vergleich zu GW170817 nicht weiter einschränken konnten; mit anderen Worten, $\chi=0$.

\section{Kapitel 4: Ein Hadron-Quark Phasenübergang in Neutronenstern- verschmelzungen}

Im zweiten Teil dieser Arbeit, Kap. 4, geht es um die Möglichkeit eines Phasenübergangs (engl. phase transition; PT) von hadronischer Materie zu Quarkmaterie. Solch ein PT, der Spekulationen zufolge bei Dichten größer als nuklearer Kerndichte auftreten könnte, könnte sowohl mit Schwerionenkollisionen als auch mit Gravitationswellen nachgewiesen werden (Hanauske et al., 2019). Basierend auf Weih et al. (2020b) wird in Kap. 4 gezeigt wie eine klare Signatur im Gravitationswellensignal entsteht, falls solch ein PT tatsächlich während der Verschmelzung zweier Neutronensterne auftreten sollte.

Hierzu wird eine spezielle Zustandsgleichung benutzt, die auf dem Modell von Tolos et al. $(2017 \mathrm{a}, \mathrm{b})$ basiert. Bei einer Dichte von $2.085 \rho_{\text {sat }}$, wobei $\rho_{\text {sat }} \approx 0.16 \mathrm{fm}^{-3}$ die nukleare Kerndichte ist, wird der Zustandsgleichung per Hand ein Phasenübergang hinzugefügt. Die Parameter werden dabei so gewählt, dass der spezielle Fall des verspäteten Phasenübergangs (engl. delayed phase transition; DPT) modelliert werden kann. Der DPT unterscheidet sich von anderen Möglichkeiten (Most et al., 2019a; Bauswein et al., 2019) für den Ausgang einer Neutronensterverschmelzung (s. Abb. 4.1) dadurch, dass nach der Verschmelzung erst das graduelle Ansteigen der Dichte zu dem PT führt. Dieser Phasenübergang führt dann zu einem Kollaps des HMNSs, der allerdings durch eine steife Quarkphase aufgehalten wird. So entsteht also kein schwarzes Loch, sondern ein zumindest metastabiler Neutronenstern mit einem Kern aus Quarkmaterie. Dieses Endprodukt wird als hypermassiver Hybridstern (HMHS) bezeichnet.

Die thermodynamischen Eigenschaften dieses HMHS unterscheiden sich signifikant von denen eines HMNS, wie in Hanauske et al. (2021) gezeigt. Der Quarkkern, der etwa $20 \%$ des HMHS ausmacht, weist deutlich höhere Dichten als der rein hadronische Kern eines HMNSs auf. Außerdem sind nicht nur die Temperaturmaxima, die für einen HMNS typisch sind (Hanauske et al., 2017a; Kastaun et al., 2017), zu sehen, sondern auch ein weiterer Ring erhöhter Temperatur, der durch den PT entsteht. Abb. 4.6 zeigt diese Unterschiede.

Die erhöhte Dichte geht einher mit einem kompakteren Stern, dessen Rotationsgeschwindigkeit höher ist als die des HMNSs vor dem teilweisen Kollaps. Dies führt zu einer höheren Frequenz in dem Gravitationswellensignal, dass durch die Rotation des HMHSs erzeugt wird. Da das Verschmelzungsprodukt zuerst ein HMNS war und dann zu dem kompakteren HMHS kollabierte, entstehen zwei dominante Frequenzen. Das Spektrum des Gravitationswellensignals (Abb. 4.9) zeigt dann zwei Spitzen, eine für die dominante hadronische und eine für die Frequenz aus der Quarkphase. Im rein hadronischen Fall (ohne PT) entsteht nur eine einzige Spitze (Bauswein \& 
Janka, 2012b; Takami et al., 2014, 2015; Bernuzzi et al., 2015; Rezzolla \& Takami, 2016). Diese Doppelspitze im Spektrum des Gravitationswellensignals ist eine eindeutige Signatur dafür, dass eine Phasenübergang hin zu Quarkmaterie stattfand. Mit zukünftigen Gravitationswellendetektoren könnte ein solch hoch-frequentes Signal gemessen werden (Punturo et al., 2010; Abbott \& et al., 2017).

Eine weitere Signatur für den PT kann in der $\ell=2, m=1$ Mode des Gravitatioswellensignals gefunden werden. Normalerweise hat diese Mode eine vernachlässigbar kleine Amplitude (im Vergleich zur dominanten $\ell=2=m$ Mode). Im DPT Szenario tritt jedoch durch den Kollaps zum HMHS eine starke Asymmetrie auf. Diese Asymmetrie ist bereits in minimaler Form während der HMNS-Phase präsent. Durch den teilweisen Kollaps wird sie drastisch verstärkt. Diese Verstärkung wirkt sich auf die produzierten Gravitationswellen aus wie in Abb. $4.10 \mathrm{zu}$ sehen ist. Zwar ist diese verstärkte Mode ein weiterer Hinweis auf eine PT, allerdings ist die Amplitude immer noch mindestens eine Größenordnung kleiner als die der Hauptmode, weshalb eine Detektion in naher Zukunft eher unwahrscheinlich ist.

\section{Kapitel 5: Strahlungstransport für numerische Astrophysik}

Das letzte Kapitel dieser Arbeit befasst sich mit Strahlungstransport in der numerischen Astrophysik. Strahlung in Form von Photonen spielt eine Rolle in Akkretionsscheiben um schwarze Löcher Zanotti et al. (2011); Fragile et al. (2012); Roedig et al. (2012); Sądowski et al. (2013); Fragile et al. (2014); McKinney et al. (2014) oder in der Dynamik von relativistischen Jets. Die Interaktionen eines Fluids mit Neutrinos in Form von Emission, Absorption und Streuung zählen ebenfalls zur Kategorie des Strahlungstransports. So sind Neutrinos verantwortlich für den Explosionsmechanismus in Kernkollaps-Supernovae. In Neutronensternverschmelzungen verändern sie die Komposition des ausgeworfenen Materials, was wiederum Einfluss auf die Nukleosynthese der schweren Elemente in unserem Universum hat (Cowperthwaite et al., 2017; Drout et al., 2017). Auch die Produktion eines SGRBs scheint mit der Interaktion zwischen Neutrinos und Fluid verbunden zu sein, wobei die Neutrinos die Polregion über dem HMNS von Materie säubern könnten, um somit eine Art Trichter für den Ausbruch eines relativistischen Jets zu fördern (Fujibayashi et al., 2017).

Das Modellieren von Strahlung wird durch die zugrunde liegende Boltzmanngleichung bestimmt, welche eine sieben-dimensionale Differential-Integralgleichung darstellt. Eine numerische Lösung dieser Gleichung entzieht sich jeglicher Rechenkapazitäten, weshalb approximative Methoden wie entwickelt wurde. Die M1-Methode, beispielsweise, stellt einen guten Kompromiss zwischen Genauigkeit und Berechenbarkeit dar und kommt tatsächlich in vielen Teilgebieten der numerischen Astrophysik zur Anwendung (z.B. in Fragile et al., 2012; McKinney et al., 2014; O'Connor, 2015; Sekiguchi et al., 2016; Foucart et al., 2015; Skinner et al., 2019). Diese Methode entwickelt von Thorne (1981) - beruht auf der Zeitentwicklung der niedrigsten Momente der Verteilungsfunktion der Strahlungsteilchen. Dies führt zu einer unendlichen Reihe von partiellen Differentialgleichung, wobei die $i$ te Gleichung für das $i$ te Moment vom $i+1$ ten Moment abhängt. Thornes Formalismus schneidet diese Reihe ab einer gegebenen Ordnung ab und schließt das Gleichungssystem durch eine analytische approximative Gleichung für das fehlende $i+1$ te Moment. Die M1-Methode entwickelt das nullte und erste Moment, also die Strahlungsenergiedichte und die Strahlungsimpulsdichte. Der Drucktensor, das zweite Moment, wird dann durch eine physikalisch motivierte analytische Gleichung zur Verfügung gestellt (Minerbo, 1978; Levermore, 1984). 
Innerhalb dieser Arbeit und basierend auf Weih et al. (2020c) wurde die M1Methode in dem neuen Code FRAC implementiert. Die Implementierung folgt der von Foucart et al. (2015), welche wiederum auf Shibata et al. (2011) basiert. Der einzige Unterschied ist die Anwendung einer IMEX-Methode (Pareschi \& Russo, 2005), die eine stabile Zeitentwicklung trotz steifer Quellterme garantiert. Die Details der Implementierung werden in Abschn. 5.1.1 präsentiert. Der darauffolgende Abschnitt verifiziert die Implementierung mit einer Reihe standardisierter Tests.

FRAC wird mit dem Code für allgemein-relativistische Magneto-Hydrodynamik BHAC (Porth et al., 2017) gekoppelt. Dadurch kann ein Fluid simuliert werden, welches mit Strahlung wechselwirkt. Genauer gesagt wird das Problem der kugelsymmetrischen Akkretion auf ein schwarzes Loch simuliert. In der Abwesenheit von Strahlung ist dieses Problem als Michel-Problem bekannt Michel (1972). Durch mehrere Simulationen, die einen großen Parameterraum abdecken, kann folgende Gleichung hergeleitet werden:

$$
\epsilon=7.41 \times 10^{-7}\left(\frac{T_{0}}{10^{6} \mathrm{~K}}\right)^{0.22}\left(\frac{L}{L_{\odot}}\right)^{0.48}\left(\frac{M}{M_{\odot}}\right)^{0.48} .
$$

Diese Relation erlaubt es die Akkretionseffizienz, $\epsilon$, in Abhängigkeit von den Observablen Temperatur, $T_{0}$, und Leuchtkraft, $L$, der Akkretionsscheibe und der Masse des schwarzen Lochs, $M$, zu berechnen.

Diese Gleichung beruht auf der numerischen Lösung des Michel-Problems, welche eine Gleichgewichtslösung zwischen Fluid und Strahlungsfeld darstellt. In Abschn. 5.1.3 wird zusätzlich gezeigt, dass diese Gleichgewichtslösung stabil unter Perturbationen ist.

Es ist allerdings bekannt, dass die M1-Methode unter systematischen Fehlern leidet. Diese werden durch die ad-hoc-Formulierung des Drucktensors verursacht. Andere Ungenauigkeiten, wie das Kreuzende-Strahlen-Problem, gehen darauf zurück, dass Strahlung innerhalb der M1-Methode wie ein makroskopisches Fluid behandelt wird. Akkuratere Methode wie beispielsweise Monte-Carlo-Methoden sind allerdings oft mit zu hohen Rechenkosten verbunden. In Abschn. 5.2 wird deshalb eine neue Methode für Strahlungstransport präsentiert, die bei vergleichbaren Rechenkosten genauere Ergebnisse als M1 liefert. Diese Gitter-Boltzmann Methode (engl. Lattice Boltzmann, LB) basiert auf der klassischen LB Methode, welche routinemäßig in der numerischen Strömungsmechanik eingesetzt wird (für einen Überblick siehe z.B. Succi et al., 2010; Krüger et al., 2017; Succi, 2018).

Die Methode beruht darauf, dass eine Quadratur hergeleitet wird, die es erlaubt die makroskopischen Momente bis zu einer gewünschten Ordnung exakt aus nur einer geringen Anzahl an diskreten Verteilungsfunktionen herzuleiten. Die klassische LB Gleichung folgt dann als ein Gleichungssystem mit $N_{\text {pop }}$ Gleichungen für genau so viele diskrete Verteilungsfunktion. Jede Population dieser Verteilungsfunktionen ist dann auf einem Gitter definiert und erlaubt die Propagation von Strahlungsteilchen in genau eine Richtung. Das Gleichungssystem wird dann mit Hilfe des strömen-undkollidieren Ansatzes gelöst.

Um dieselbe Methode für Strahlungstransport in der numerischen Astrophysik zu verwenden bedarf es einiger Modifikationen. Vor allem die Herleitung einer Quadraturregel kann nicht wie für klassisches LB auf Erhaltungsgesetzen basieren. Dies liegt an dem Fehlen eben dieser Gesetze für Strahlung, die dynamisch mit einem Fluid Energie und Impuls austauscht. Stattdessen werden verschiedene Quadraturen basierend auf Symmetrie- und Isotropieannahmen hergeleitet. Des Weiteren muss die LB Gleichung modifiziert werden, um Emission, Absorption und Streuung von Strahlung zu 
integrieren. Die diskretisierte LB Gleichung für Strahlungstransport lautet dann

$$
I_{(i)}\left(\boldsymbol{r}+\hat{\boldsymbol{n}}_{(i)} \Delta t, t+\Delta t\right)=I_{(i)}(\boldsymbol{r}, t)-\kappa_{0} \Delta t\left(I_{(i)}(\boldsymbol{r}, t)-I_{(i)}^{\mathrm{eq}}(\boldsymbol{r}, t)\right)+\Delta t S_{(i)}(\boldsymbol{r}, t) .
$$

Dies ist ein System von Entwicklungsgleichung für die $N_{\text {pop }}$ diskreten spezifischen Intensitäten, $I_{(i)}$, wobei jede Intensität nur von der Position im Raum, r, und der Zeit, $t$, abhängt. Der Index $(i)$ gibt dabei an in welche Richtung $\hat{\boldsymbol{n}}_{(i)}$ die Strahlung propagiert. Die Zeitentwicklung wird bestimmt durch den Equilibrierungsterms, der proportional zur Streuopazität $\kappa_{0}$ ist, und dem Quellterms $S_{(i)}$, der Emission und Absorption beinhaltet. Das Lösen der LB Gleichung erfolgt wie auch im klassischen Fall über die strömen-und-kollidieren-Methode.

Nachdem diese neue LB-Methode implementiert wurde, wird wiederum über eine Reihe standardisierter Tests verifiziert, dass dieses mal nicht nur die Implementierung, sondern auch die Methodik an sich korrekt ist. Dabei wird stets mit der M1-Methode verglichen. Tatsächlich zeigt die neue LB-Methode genauere Ergebnisse für den Kreuzende-Strahlen Test und ist in der Lage den Drucktensor in allen Fällen korrekt zu rekonstruieren. Dies zeigt sich besonders deutlich in Simulationen von relativistischen Jets (Abschn. 5.2.4). Durch einen Schock, der dadurch entsteht, dass der Jet auf das umgebende Medium trifft, entstehe zwei Strahlenbündel im Strahlungsfeld. Diese beiden Strahlungsbündel treffen sich am Ende des Schocks und - im Fall von M1 verbinden sich zu einem einzigen Strahl. Dies führt zu einer erhöhten Energiedichte entlang der zentralen Achse des Jets. Durch die Rückkopplung mit dem Fluid wird diese Energie in den Jet eingespeist, was wiederum die Ausbreitungsgeschwindigkeit des Jets erhöht. Im Fall von LB ist dies nicht zu sehen, da die beiden Strahlenbündel sich am Ende des Schocks nicht verbinden, sondern korrekterweise durcheinander hindurch gehen. Somit verteilt sich die Energie auf eine breitere Fläche (s. Abb. 5.27).

Diese Simulation dient nicht nur dazu die intrinsischen Fehler der M1-Methode aufzuzeigen, sondern auch als Beweis dafür, dass die neue LB-Methode tatsächlich für numerische Astrophysik geeignet ist.

\section{Fazit}

In dieser Arbeit wurde in drei Kapiteln gezeigt wie moderne astrophysikalische Beobachtungen durch theoretische Berechnungen ergänzt werden. Dieses Zusammenspiel hat vor allem seit GW170817 an Bedeutung gewonnen und stellt für die Zukunft einen der vielversprechendsten Wege dar, um die fundamentalen Gesetze unseres Universums zu entschlüsseln. Kurz vor Vollendung dieser Arbeit beendete LVC den dritten Beobachtungsdurchlauf (Abbott et al., 2020b) und bereitet sich nun auf zukünftige Beobachtungen mit noch sensibleren Detektoren vor. Dabei sagt LVC eine aussichtsvolle Zukunft für die Multimessenger-Astronomie voraus (The LIGO Scientific Collaboration \& the Virgo Collaboration, 2020). Diese Arbeit leistet dabei hoffentlich einen kleinen Beitrag zu dieser Zukunft. 


\section{Professional experience}

2018-now Physicist, Johann-Wolfgang-Goethe University, Frankfurt, Germany.

Research in high-energy astrophysics using state-of-the-art computational physics and data analysis methods as part of my PhD studies.

2014-2015, Tutor, Johann-Wolfgang-Goethe University, Frankfurt, Germany.

2017-2018 Teaching and tutoring undegraduate students in exercise lessons for theoretical physics and astrophysics.

2015-2018 Team leader, Kinopolis, Hanau, Germany.

Supervising a team of up to 30 service employees, managing and ensuring the smooth day-to-day operations in a cinema with up to 3500 customers daily.

\section{Education}

2018-now PhD, Johann-Wolfgang-Goethe University, Frankfurt, Germany.

Expected to graduate in 2021 with a thesis titled "Multimessenger approaches to exploring dense matter in neutron stars" under the supervision of Prof. Luciano Rezzolla.

2015-2017 Master of Science, Johann-Wolfgang-Goethe University, Frankfurt, Germany. Master degree in physics in November 2017 graduating with a thesis titled "Equilibria and dynamics of rotating relativistic stars" under the supervision of Prof. Luciano Rezzolla. Overall grade: 1.0

2012-2015 Bachelor of Science, Johann-Wolfgang-Goethe University, Frankfurt, Germany. Bachelor degree in physics in July 2015 graduating with a thesis titled "Compact stars with cosmological constant" under the supervision of Prof. Jürgen SchaffnerBielich. Overall grade: 1.1

2012 English Language School, EF International Language Center, Toronto, Canada.

2003-2011 High School, Spessart-Gymnasium, Alzenau, Germany. Overall Grade: 1.5

\section{Highlights}

Excellence "In recognition of outstanding achievements in the doctoral thesis project", $2500 €$ Award awarded by Stiftung Giersch.

Scholarship "To promote scientific education", 3-year funding provided by GSI-F\&E. 
Public My research has attracted a lot of media attention in national and international Outreach media (e.g., FAZ, Spektrum), most notably, my vizualisations for the publication of the first image of a black hole by the EHT collaboration. These visualizations have been viewed several Million times on YouTube and other social media channels.

Reviewing Expert reviewer for research articles submitted to Astrophysical Journal and IOPScience.

Grants Contributed to a number of applications for computing time leading to more than 100 Million CPU-hours on some of Europe's largest computer clusters with an estimated value of several Million $€$.

Software Main developer and maintainer of the radiative transfer code FRAC, developer of Engineering the open-source finite-volume code BHAC.

Numerical Main developer of the Lattice Boltzmann method for radiative transfer in comPhysics putational astrophysics.

\section{Languages}

German Native.

English $\quad$ C2 written and spoken.

Spanish fluent written and spoken.

\section{Computer Skills}

Programming Advanced: Python (NumPy, SciPy, Pyplot, Jupyter/Ipython), C/C++, Fortran. Basic: Shell Scripting, CMake, Haskell, SQL, Machine Learning (TensorFlow). Familiar: R, Cotlin.

Scientific Mathematica, data visualization (with Pyplot, Visit, Paraview, and Amira), parallel programming using MPI and OpenMP, signal processing, state-of-the-art numerical methods.

Data analysis Experienced in big-data analysis using HDF5, Python, Cython, Machine Learning (DNN), parallel programming, algorithmic analysis, and creative visualization techniques.

Others $\quad$ MS Office, UNIX Operating System, Latex, GIT.

\section{Publications}

- Weih, L. R., Most, E. R., \& Rezzolla, L., On the Stability and Maximum Mass of Differentially Rotating Relativistic Stars, Mon. Not. R. Astron. Soc., 473, L126 (2018). 
- Rezzolla, L., Most, E. R., \& Weih, L. R., Using Gravitational-wave Observations and Quasi-universal Relations to Constrain the Maximum Mass of Neutron Stars, Astrophys. J. Lett., 852, L25 (2018).

- Most, E. R., Weih, L. R., Rezzolla, L., \& Schaffner-Bielich, J., New Constraints on Radii and Tidal Deformabilities of Neutron Stars from GW170817, Phys. Rev. Lett., 120, 261103 (2018).

- Weih, L. R., Most, E. R., \& Rezzolla, L., Optimal Neutron-star Mass Ranges to Constrain the Equation of State of Nuclear Matter with Electromagnetic and Gravitational-wave Observations, Astrophys J., 881, 73 (2019).

- Weih, L. R., Hanauske, M., \& Rezzolla, L., Postmerger Gravitational-Wave Signatures of Phase Transitions in Binary Mergers, Phys. Rev. Lett., 124, 171103 (2020).

- Most, E. R., Weih, L. R., \& Rezzolla, L., The Heavier the Better: How to Constrain Mass Ratios and Spins of High-Mass Neutron-Star Mergers, Mon. Not. R. Astron. Soc., 496, L16 (2020).

- Weih, L. R., Olivares, H., \& Rezzolla, L., Two-moment scheme for general-relativistic radiation hydrodynamics: a systematic description and new applications, Mon. Not. R. Astron. Soc., 495, 2285 (2020).

- Most, E. R., Papenfort, L. J., Weih, L. R., \& Rezzolla, L., A Lower Bound on the Maximum Mass if the Secondary in GW190814 was Once a Rapidly Spinning Neutron Star, Mon. Not. R. Astron. Soc., 499, L82 (2020)

- Weih, L. R., Gabbana, A., Simeoni, D., Rezzolla, L., Succi, S., \& Tripiccione, R., Beyond moments: relativistic lattice Boltzmann methods for radiative transport in computational astrophysics, Mon. Not. R. Astron. Soc., 498, 3374 (2020).

- Hanauske, M., Weih, L. R., Stöcker, H., \& Rezzolla, L., Metastable hypermassive hybrid stars as neutron-star merger remnants, European Physical Journal, in press (2021).

\section{Conferences and Workshops}

Seminar HGS-HIRe softskill course on "Leadership and career development", online, May 2020 .

Conference "The first compact star merger event - Implications for nuclear and particle physics", Trento (Italy), October 2019.

Conference "MICRA2019 - Microphysics in computational astrophysics", Jena (Germany) August 2019.

Seminar HGS-HIRe softskill course on "Leading teams in research environments", Buchenau (Germany), June-July 2019.

Workshop Winter school on "Introduction to Machine Learning for scientists", Leuven (Belgium), March 2019.

Workshop Training course on "Advanced C++ with focus on software engineering", Stuttgart (Germany), May 2019.

Seminar HGS-HIRe softskill course on "Making an impact as an effective researcher", 
Buchenau (Germany), October 2018.

Workshop Summer school on "Physics of macronovae", Stockholm (Sweden), June 2018.

Conference "Fire and ice: hot QCD meets cold and dense matter", Saariselkä (Finland), April 201.8

Workshop Training course on "Introduction to parallel programming with MPI and OpenMP", Jülich (Germany), November 2017.

Workshop Training course on "Vectorisation and portable programming using OpenCL", Jülich (Germany), November 2017.

Workshop Summer school on "Neutron stars: theory, observations and gravitational waves emission", Sofia (Bulgaria), September 2017.

\section{Presentations}

Invited Talk "Postmerger gravitational-wave signatures of phase transitions in binary mergers", New Work (online seminar), June 2020.

Contributed Talk "Optimal neutron-star mass ranges for constraining the equation of state", Trento (Italy), October 2019.

Contributed Talk "Optimal neutron-star mass ranges for constraining the equation of state", Jena (Germany), August 2019.

Invited Talk "Joint Astro-Nuclear-Physics Colloquium: Constaining the equation of state in the aftermath of GW170817", Frankfurt a. M. (Germany), July 2018.

Contributed Talk "Constraints on neutron star properties from the gravitational-wave observation GW170817”, Saariselkä (Finland), April 2018.

Poster "Stability criterion for differentially rotating neutron stars", Sofia (Bulgaria) September 2017. 


\section{Selbstständigkeitserklärung}

Ich erkläre hiermit, dass ich die vorgelegte Dissertation über

"Multi-messenger Approaches to Exploring Dense Matter in Neuton Stars"

selbstständig angefertigt und mich anderer Hilfsmittel als der in ihr angegebenen nicht bedient habe, insbesondere, dass alle Entlehnungen aus anderen Schriften mit Angabe der betreffenden Schrift gekennzeichnet sind.

Ich versichere, die Grundsätze der guten wissenschaftlichen Praxis beachtet, und nicht die Hilfe einer kommerziellen Promotionsvermittlung in Anspruch genommen zu haben.

Frankfurt am Main, den 